\title{
Highly Carbon-selective Monofluoromethylation of $\beta$-Ketoesters with Fluoromethyl iodide
}

Tianqi Ding, Lvqi Jiang*, Jie Yang, Yimin Xu, Guixiang Wang and Wenbin Yi*

School of Chemical Engineering, Nanjing University of Science and Technology,

Nanjing 210094, China

\section{Table of Contents}

1. General information

2. Preparation of the $\beta$-ketoester compounds

3. Table S1: Optimization of reaction conditions

4. General procedure for carbon-selective monofluoromethylation of $\beta$-ketoesters $2 \mathrm{a}-\mathrm{w}$

5. Synthesis of product $3 y$

6. General procedure for carbon-selective monofluoromethylation of malonates

7. Gram-scale Reactions

8. Synthesis of compounds 5 and 6

9. Table S2: Survey for DAAA reaction of product 3r

10. DFT calculation 


\section{General information}

All chemical reagents are obtained from commercial suppliers and used without further purification. All known compounds are characterized by ${ }^{1} \mathrm{H}$ NMR and ${ }^{19} \mathrm{~F}$ NMR. All unknown compounds are characterized by ${ }^{1} \mathrm{H}$ NMR, ${ }^{13} \mathrm{C}$ NMR, ${ }^{19} \mathrm{~F}$ NMR and HRMS. Analytical thin-layer chromatography are performed on glass plates precoated with silica gel impregnated with a fluorescent indicator $(254 \mathrm{~nm})$, and the plates are visualized by exposure to ultraviolet light. Mass spectra are taken on a Finnigan TSQ Quantum-MS instrument in the electrospray ionization (ESI) mode. ${ }^{1} \mathrm{H},{ }^{13} \mathrm{C}$ and ${ }^{19} \mathrm{~F}$ NMR spectra were recorded on a $500 \mathrm{MHz}$ Bruker DRX 500 and data were reported as follows: chemical shifts in ppm from tetramethylsilane as an internal standard in $\mathrm{CDCl}_{3}$, integration, multiplicity ( $\mathrm{s}=$ singlet, $\mathrm{d}=$ doublet, $\mathrm{t}=$ triplet, $\mathrm{q}=$ quartet, $\mathrm{dd}=$ doublet-doublet, $\mathrm{m}=$ multiplet, $\mathrm{br}=$ broad), coupling constants $(\mathrm{Hz})$, and assignment. ${ }^{19} \mathrm{~F}$ NMR chemical shifts were determined relative to $\mathrm{CFCl}_{3}$ as inter standard. GC analyses are performed on an Agilent 7890A instrument (Column: Agilent 19091J-413: $30 \mathrm{~m} \times 320$ $\mu \mathrm{m} \times 0.25 \mu \mathrm{m}$, carrier gas: $\mathrm{H}_{2}$, FID detection. GC-MS data was recorded on a ISQ LT Single Quadrupole Mass Spectrometer, coupled with a Trace 1300 Gas Chromatograph (Thermo Fisher Scientific). High resolution mass spectral data were acquired on Waters Micromass GCT Premier spectrometer (electrospray ionization: EI). 


\section{Preparation of the $\beta$-ketoester compounds}

Method $A^{1}$ :

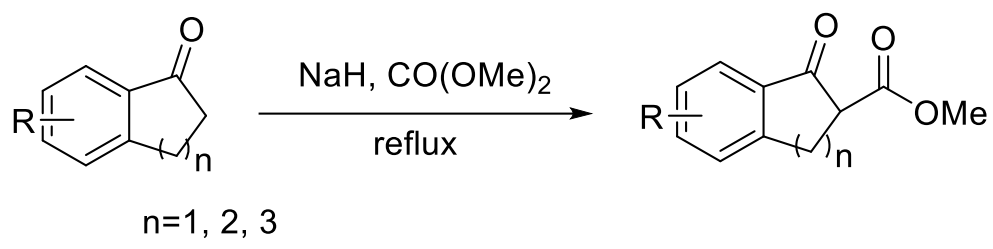

To the suspension of $\mathrm{NaH}$ (12.5 mmol, 2.5 equiv, $60 \%$ dispersion in mineral oil $)$ in dimethyl carbonate $(1.0 \mathrm{M}, 5 \mathrm{~mL})$ was added the solution of tetralone $(5 \mathrm{mmol})$ in dimethyl carbonate $(1.0 \mathrm{M}, 5 \mathrm{~mL})$ dropwise at room temperature. The resulting mixture was heated to reflux for $12 \mathrm{~h}$. After cooling to room temperature, the reaction mixture was poured into ice water and extracted with EtOAc (three times). The combined organic layer was washed with brine, dried over $\mathrm{MgSO}_{4}$, and concentrated in vacuo. The residue was purified by column chromatography on silica gel (ethyl acetate/ petroleum ether $=1: 8)$ to give the product $2 \mathrm{a}$ as a yellow solid.

\section{methyl 1-oxo-1,2,3,4-tetrahydronaphthalene-2-carboxylate $2 \mathrm{a}^{1}$}<smiles>COC(=O)C1CCc2ccccc2C1=O</smiles>

Yellow solid. Eluent: ethyl acetate/ petroleum ether (1:8). ${ }^{1} \mathrm{H} \mathrm{NMR}\left(500 \mathrm{MHz}, \mathrm{CDCl}_{3}\right)$ : The compound exists as a 1:1.2 mixture of keto/enol tautomer. Signals corresponding to the keto tautomer: 8.04 (d, $J$ $=7.8 \mathrm{~Hz}, 1 \mathrm{H}), 7.48(\mathrm{dd}, J=10.7,4.2 \mathrm{~Hz}, 1 \mathrm{H}), 7.27-7.21(\mathrm{~m}, 2 \mathrm{H}), 3.77(\mathrm{~s}, 3 \mathrm{H}), 3.62(\mathrm{dd}, J=10.5,4.7$ Hz, 1H), $3.09-2.93(\mathrm{~m}, 2 \mathrm{H}), 2.49(\mathrm{dtd}, J=14.9,10.1,4.8 \mathrm{~Hz}, 1 \mathrm{H}), 2.38-2.30(\mathrm{~m}, 1 \mathrm{H})$; Signals corresponding to the enol tautomer: $\delta 12.43(\mathrm{~s}, 1 \mathrm{H}), 7.80(\mathrm{~d}, J=7.6 \mathrm{~Hz}, 1 \mathrm{H}), 7.34-7.28(\mathrm{~m}, 2 \mathrm{H}), 7.16$ $(\mathrm{d}, J=7.2 \mathrm{~Hz}, 1 \mathrm{H}), 3.81(\mathrm{~s}, 3 \mathrm{H}), 2.80(\mathrm{t}, J=7.8 \mathrm{~Hz}, 2 \mathrm{H}), 2.56(\mathrm{t}, J=7.8 \mathrm{~Hz}, 2 \mathrm{H}) ;{ }^{13} \mathrm{C} \mathrm{NMR}(126 \mathrm{MHz}$, $\mathrm{CDCl}_{3}$ ) Signals corresponding to both tautomers: $\delta 194.2$ (s), 174.2 (s), 171.7 (s), 166.2 (s), 144.8 (s), 140.5 (s), 135.0 (s), 132.8 (s), 131.6 (s), 131.0 (s), 129.9 (s), 128.8 (s), 128.5 (s), 128.0 (s), 127.7 (s), 125.4 (s), 97.9 (s), 55.5 (s), 53.4 (s), 52.7 (s), 28.8 (s), 28.7 (s), 27.5 (s), 21.6 (s). 
methyl 7-methyl-1-oxo-1,2,3,4-tetrahydronaphthalene-2-carboxylate $2 b^{2}$<smiles>COC(=O)C1CCc2ccc(C)cc2C1=O</smiles>

Following the Method A: Using 7-methyl-3,4-dihydronaphthalen-1(2H)-one instead of tetralone. Yellow solid. Eluent: ethyl acetate/ petroleum ether (1:8). ${ }^{1} \mathrm{H} \mathrm{NMR}\left(500 \mathrm{MHz}, \mathrm{CDCl}_{3}\right)$ : The compound exists as a 1:1.4 mixture of keto/enol tautomer. Signals corresponding to the keto tautomer: 7.84 (s, $1 \mathrm{H}), 7.31-7.28(\mathrm{~m}, 1 \mathrm{H}), 7.05(\mathrm{~d}, J=7.6 \mathrm{~Hz}, 1 \mathrm{H}), 3.77(\mathrm{~s}, 3 \mathrm{H}), 3.59(\mathrm{dd}, J=10.4,4.7 \mathrm{~Hz}, 1 \mathrm{H}), 3.05-$ $2.87(\mathrm{~m}, 2 \mathrm{H}), 2.47(\mathrm{dtd}, J=14.4,9.9,4.7 \mathrm{~Hz}, 1 \mathrm{H}), 2.35(\mathrm{~s}, 3 \mathrm{H}), 2.35-2.30(\mathrm{~m}, 1 \mathrm{H})$; Signals corresponding to the enol tautomer: $\delta 12.44(\mathrm{~s}, 1 \mathrm{H}), 7.62(\mathrm{~s}, 1 \mathrm{H}), 7.13(\mathrm{~d}, J=7.9 \mathrm{~Hz}, 2 \mathrm{H}), 3.82(\mathrm{~s}, 3 \mathrm{H})$, $2.75(\mathrm{t}, J=7.8 \mathrm{~Hz}, 2 \mathrm{H}), 2.55(\mathrm{t}, J=7.8 \mathrm{~Hz}, 2 \mathrm{H}), 2.35(\mathrm{~s}, 3 \mathrm{H}) ;{ }^{13} \mathrm{C} \mathrm{NMR}\left(126 \mathrm{MHz}, \mathrm{CDCl}_{3}\right)$ Signals corresponding to both tautomers: $\delta 194.4(\mathrm{~s}), 174.2$ (s), 171.8 (s), 166.4 (s), 141.9 (s), 137.7 (s), 137.6 (s), $137.2(\mathrm{~s}), 136.0(\mathrm{~s}), 132.6$ (s), 132.4 (s), 130.8 (s), 129.8 (s), 128.8 (s), 128.4 (s), 125.9 (s), 97.9 (s), $55.6(\mathrm{~s}), 53.4(\mathrm{~s}), 52.7$ (s), $28.4(\mathrm{~s}), 28.3(\mathrm{~s}), 27.6(\mathrm{~s}), 22.2(\mathrm{~s}), 22.0(\mathrm{~s}), 21.8$ (s).

methyl 6-methoxy-1-oxo-1,2,3,4-tetrahydronaphthalene-2-carboxylate $2 c^{3}$<smiles>COC(=O)C1CCc2cc(OC)ccc2C1=O</smiles>

Following the Method A: Using 6-methoxy-3,4-dihydronaphthalen-1(2H)-one instead of tetralone. Yellow oil. Eluent: ethyl acetate/ petroleum ether (1:8). ${ }^{1} \mathrm{H}$ NMR (500 MHz, $\left.\mathrm{CDCl}_{3}\right)$ : The compound exists as a 4:1 mixture of keto/enol tautomer. Signals corresponding to the keto tautomer: 8.00 (d, $J=$ $8.8 \mathrm{~Hz}, 1 \mathrm{H}), 6.82(\mathrm{dd}, J=8.8,2.5 \mathrm{~Hz}, 1 \mathrm{H}), 6.69$ (d, $J=7.6 \mathrm{~Hz}, 1 \mathrm{H}), 3.84$ (s, 3H), 3.77 (s, 3H), 3.57 (dd, $J=10.2,4.7 \mathrm{~Hz}, 1 \mathrm{H}), 3.07-2.89(\mathrm{~m}, 2 \mathrm{H}), 2.51-2.41(\mathrm{~m}, 1 \mathrm{H}), 2.38-2.28(\mathrm{~m}, 1 \mathrm{H})$; Signals corresponding to the enol tautomer: $\delta 12.46(\mathrm{~s}, 1 \mathrm{H}), 7.72(\mathrm{~d}, J=8.6 \mathrm{~Hz}, 1 \mathrm{H}), 6.78(\mathrm{dd}, J=8.6,2.5 \mathrm{~Hz}$, 1H), $6.71(\mathrm{~d}, J=7.6 \mathrm{~Hz}, 1 \mathrm{H}), 3.82(\mathrm{~s}, 3 \mathrm{H}), 3.80(\mathrm{~s}, 3 \mathrm{H}), 2.77(\mathrm{t}, J=7.7 \mathrm{~Hz}, 2 \mathrm{H}), 2.54(\mathrm{t}, J=7.7 \mathrm{~Hz}$, $2 \mathrm{H}) ;{ }^{13} \mathrm{C}$ NMR $\left(126 \mathrm{MHz}, \mathrm{CDCl}_{3}\right.$ ) Signals corresponding to both tautomers: $\delta 192.9$ (s), 174.2 (s), 172.0 (s), 166.6 (s), 165.1 (s), 162.6 (s), 147.3 (s), 142.9 (s), 131.4 (s), 127.3 (s), 126.3 (s), 123.9 (s), $114.6(\mathrm{~s}), 114.2$ (s), 113.7 (s), 112.8 (s), 95.9 (s), 56.6 (s), 56.4 (s), 55.3 (s), 53.4 (s), 52.6 (s), 29.3 (s), $29.1(\mathrm{~s}), 27.5(\mathrm{~s}), 21.6(\mathrm{~s})$. 
methyl 7-methoxy-1-oxo-1,2,3,4-tetrahydronaphthalene-2-carboxylate $2 d^{4}$<smiles>COC(=O)C1CCc2ccc(OC)cc2C1=O</smiles>

Following the Method A: Using 7-methoxy-3,4-dihydronaphthalen-1(2H)-one instead of tetralone. Yellow oil. Eluent: ethyl acetate/ petroleum ether (1:8). ${ }^{1} \mathrm{H}$ NMR (500 MHz, $\left.\mathrm{CDCl}_{3}\right)$ : The compound exists as a 1.2:1 mixture of keto/enol tautomer. Signals corresponding to the keto tautomer: 7.46 (d, $J$ $=2.7 \mathrm{~Hz}, 1 \mathrm{H}), 7.13-7.08(\mathrm{~m}, 1 \mathrm{H}), 6.83(\mathrm{dd}, J=8.2,2.6 \mathrm{~Hz}, 1 \mathrm{H}), 3.78(\mathrm{~s}, 3 \mathrm{H}), 3.74(\mathrm{~s}, 3 \mathrm{H}), 3.58-$ $3.52(\mathrm{~m}, 1 \mathrm{H}), 2.98-2.82(\mathrm{~m}, 2 \mathrm{H}), 2.46-2.37$ (m, 1H), 2.29 (ddt, J = 7.9, 5.6, 4.7 Hz, 1H); Signals corresponding to the enol tautomer: $\delta 12.42(\mathrm{~s}, 1 \mathrm{H}), 7.30(\mathrm{~d}, J=2.5 \mathrm{~Hz}, 1 \mathrm{H}), 7.02(\mathrm{dd}, J=8.4,2.7 \mathrm{~Hz}$, 2H), $3.78(\mathrm{~s}, 3 \mathrm{H}), 3.74(\mathrm{~s}, 3 \mathrm{H}), 2.68(\mathrm{t}, J=7.8 \mathrm{~Hz}, 2 \mathrm{H}), 2.49(\mathrm{t}, J=7.8 \mathrm{~Hz}, 2 \mathrm{H}) ;{ }^{13} \mathrm{C} \mathrm{NMR}(126 \mathrm{MHz}$, $\mathrm{CDCl}_{3}$ ) Signals corresponding to both tautomers: $\delta 194.2$ (s), 174.2 (s), 171.8 (s), 166.0 (s), 159.5 (s), 159.4 (s), 137.4 (s), 133.5 (s), 132.6 (s), 131.8 (s), 131.1 (s), 129.4 (s), 123.3 (s), 118.0 (s), 110.6 (s), 110.0 (s), 98.2 (s), 56.5 (s), 56.4 (s), 55.4 (s), 53.3 (s), 52.7 (s), 27.9 (s), 27.7 (s), 21.9 (s).

methyl 7-bromo-1-oxo-1,2,3,4-tetrahydronaphthalene-2-carboxylate $2 \mathrm{e}^{5}$<smiles>COC(=O)C1CCc2ccc(Br)cc2C1=O</smiles>

Following the Method A: Using 7-bromo-3,4-dihydronaphthalen-1(2H)-one instead of tetralone. Yellow solid. Eluent: ethyl acetate/ petroleum ether (1:8). ${ }^{1} \mathrm{H} \mathrm{NMR}\left(500 \mathrm{MHz}, \mathrm{CDCl}_{3}\right)$ : The compound exists as a 1:2 mixture of keto/enol tautomer. Signals corresponding to the keto tautomer: 8.15 (d, $J=$ $1.9 \mathrm{~Hz}, 1 \mathrm{H}), 7.59$ (dd, $J=8.2,2.1 \mathrm{~Hz}, 1 \mathrm{H}), 7.14(\mathrm{~d}, J=8.2 \mathrm{~Hz}, 1 \mathrm{H}), 3.78$ (s, 3H), 3.61 (dd, $J=9.9,4.7$ $\mathrm{Hz}, 1 \mathrm{H}), 3.04-2.90(\mathrm{~m}, 2 \mathrm{H}), 2.53-2.44(\mathrm{~m}, 1 \mathrm{H}), 2.35$ (ddd, $J=13.7,10.9,4.8 \mathrm{~Hz}, 1 \mathrm{H})$; Signals corresponding to the enol tautomer: $\delta 12.33(\mathrm{~s}, 1 \mathrm{H}), 7.91(\mathrm{~d}, J=1.7 \mathrm{~Hz}, 1 \mathrm{H}), 7.42(\mathrm{dd}, J=8.0,2.0 \mathrm{~Hz}$, $1 \mathrm{H}), 7.04(\mathrm{~d}, J=8.0 \mathrm{~Hz}, 1 \mathrm{H}), 3.83(\mathrm{~s}, 3 \mathrm{H}), 2.75(\mathrm{t}, J=7.8 \mathrm{~Hz}, 2 \mathrm{H}), 2.56(\mathrm{t}, J=7.8 \mathrm{~Hz}, 2 \mathrm{H}) ;{ }^{13} \mathrm{C} \mathrm{NMR}$ $\left(126 \mathrm{MHz} \mathrm{CDCl}_{3}\right)$ Signals corresponding to both tautomers: $\delta 192.9$ (s), $173.9(\mathrm{~s}), 171.3$ (s), 164.6 (s), 143.4 (s), 139.1 (s), 137.8 (s), 134.3 (s), 132.9 (s), 131.8 (s), 131.6 (s), 130.1 (s), 128.4 (s), 122.0 (s), $121.4(\mathrm{~s}), 98.8$ (s), 55.1 (s), $53.6(\mathrm{~s}), 52.9$ (s), 28.3 (s), 28.1 (s), 27.2 (s), $21.4(\mathrm{~s})$. 
methyl 1-oxo-2,3-dihydro-1H-indene-2-carboxylate $2 \mathrm{~h}^{1}$

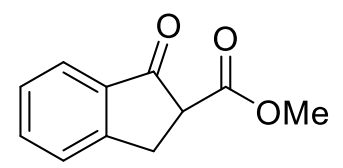

Following the Method A: Using indanone instead of tetralone. Yellow solid. Eluent: ethyl acetate/ petroleum ether (1:6). ${ }^{1} \mathrm{H}$ NMR $\left(500 \mathrm{MHz}, \mathrm{CDCl}_{3}\right)$ : The compound exists as a 4.5:1 mixture of keto/enol tautomer. Signals corresponding to the keto tautomer: $\delta 7.75(\mathrm{~d}, J=7.7 \mathrm{~Hz}, 1 \mathrm{H}), 7.61(\mathrm{t}, J=$ $7.4 \mathrm{~Hz}, 1 \mathrm{H}), 7.48$ (d, $J=7.7 \mathrm{~Hz}, 1 \mathrm{H}), 7.37$ (t, $J=7.5 \mathrm{~Hz}, 1 \mathrm{H}), 3.77(\mathrm{~s}, 3 \mathrm{H}), 3.72(\mathrm{dd}, J=8.3,4.1 \mathrm{~Hz}$, $1 \mathrm{H}), 3.55(\mathrm{dd}, J=17.2,4.0 \mathrm{~Hz}, 1 \mathrm{H}), 3.36(\mathrm{dd}, J=17.2,8.3 \mathrm{~Hz}, 1 \mathrm{H})$; Representative signals corresponding to the enol tautomer: $3.84(\mathrm{~s}, 3 \mathrm{H}), 3.49$ (s, 2H); ${ }^{13} \mathrm{C}$ NMR (126 MHz, $\left.\mathrm{CDCl}_{3}\right)$ Signals corresponding to both tautomers: $\delta 200.5$ (s), 170.6 (s), 154.7 (s), 136.6 (s), 136.3 (s), 130.5 (s), 128.9 (s), 127.9 (s), 127.6 (s), 125.8 (s), 125.7 (s), 121.8 (s), 54.2 (s), 53.8 (s), 52.3 (s), 33.6 (s), 31.4 (s).

methyl 6-methyl-1-oxo-2,3-dihydro-1H-indene-2-carboxylate $2 \mathrm{i}^{6}$<smiles>COC(=O)C1Cc2ccc(C)cc2C1=O</smiles>

Following the Method A: Using 6-methyl-2,3-dihydro-1H-inden-1-one instead of tetralone. Yellow solid. Eluent: ethyl acetate/ petroleum ether (1:6). ${ }^{1} \mathrm{H} \mathrm{NMR}\left(500 \mathrm{MHz}, \mathrm{CDCl}_{3}\right)$ : The compound exists as a 8:1 mixture of keto/enol tautomer. Signals corresponding to the keto tautomer: $\delta 7.55(\mathrm{~s}, 1 \mathrm{H}), 7.45$ - $7.41(\mathrm{~m}, 1 \mathrm{H}), 7.38$ (d, $J=7.8 \mathrm{~Hz}, 1 \mathrm{H}), 3.78(\mathrm{~s}, 3 \mathrm{H}), 3.72$ (dd, $J=8.2,4.0 \mathrm{~Hz}, 1 \mathrm{H}), 3.49$ (dd, $J=17.1$, $3.8 \mathrm{~Hz}, 1 \mathrm{H}), 3.32(\mathrm{dd}, J=17.1,8.2 \mathrm{~Hz}, 1 \mathrm{H}), 2.39$ (s, 3H); Representative signals corresponding to the enol tautomer: $3.84(\mathrm{~s}, 3 \mathrm{H}), 3.45$ (s, 2H), 2.41 (s, 3H); ${ }^{13} \mathrm{C}$ NMR (126 MHz, $\left.\mathrm{CDCl}_{3}\right)$ Signals corresponding to both tautomers: $\delta 200.6$ (s), 170.8 (s), 152.1 (s), 138.9 (s), 137.9 (s), 136.5 (s), 127.3 (s), $125.6(\mathrm{~s}), 125.5(\mathrm{~s}), 122.2$ (s), 54.6 (s), 53.8 (s), 52.3 (s), 31.0 (s), 22.5 (s), 22.1 (s).

methyl 5-methoxy-1-oxo-2,3-dihydro-1H-indene-2-carboxylate $2 \mathrm{j}^{6}$<smiles>COC(=O)C1Cc2cc(OC)ccc2C1=O</smiles> 
Following the Method A: Using 5-methoxy-2,3-dihydro-1H-inden-1-one instead of tetralone. Yellow solid. Eluent: ethyl acetate/ petroleum ether (1:6). ${ }^{1} \mathrm{H}$ NMR $\left(500 \mathrm{MHz}, \mathrm{CDCl}_{3}\right) \delta 7.71(\mathrm{~d}, J=9.2 \mathrm{~Hz}$, 1H), $6.95-6.90(\mathrm{~m}, 2 \mathrm{H}), 3.90(\mathrm{~s}, 3 \mathrm{H}), 3.79(\mathrm{~s}, 3 \mathrm{H}), 3.73(\mathrm{dd}, J=8.2,3.9 \mathrm{~Hz}, 1 \mathrm{H}), 3.52(\mathrm{dd}, J=17.3$, $3.9 \mathrm{~Hz}, 1 \mathrm{H}), 3.32(\mathrm{dd}, J=17.3,8.2 \mathrm{~Hz}, 1 \mathrm{H}) ;{ }^{13} \mathrm{C} \mathrm{NMR}\left(126 \mathrm{MHz}, \mathrm{CDCl}_{3}\right) \delta 198.5(\mathrm{~s}), 170.9(\mathrm{~s}), 167.0$ (s), $157.8(\mathrm{~s}), 129.5$ (s), 127.5 (s), 117.1 (s), 110.7 (s), 56.8 (s), 54.4 (s), 53.8 (s), 31.4 (s).

\section{methyl 5,6-dimethoxy-1-oxo-2,3-dihydro-1H-indene-2-carboxylate $2 \mathrm{k}^{3}$}

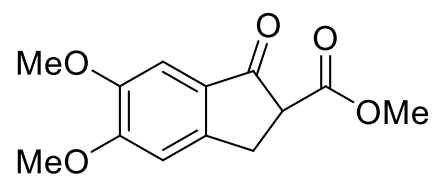

Following the Method A: Using 5,6-dimethoxy-2,3-dihydro-1H-inden-1-one instead of tetralone. Yellow solid. Eluent: ethyl acetate/ petroleum ether (1:6). ${ }^{1} \mathrm{H} \mathrm{NMR}\left(500 \mathrm{MHz}, \mathrm{CDCl}_{3}\right) \delta 7.16(\mathrm{~s}, 1 \mathrm{H})$, $6.90(\mathrm{~s}, 1 \mathrm{H}), 3.97(\mathrm{~s}, 3 \mathrm{H}), 3.89(\mathrm{~s}, 3 \mathrm{H}), 3.77(\mathrm{~s}, 3 \mathrm{H}), 3.71(\mathrm{dd}, J=7.9,3.5 \mathrm{~Hz}, 1 \mathrm{H}), 3.44(\mathrm{dd}, J=17.0$, $3.5 \mathrm{~Hz}, 1 \mathrm{H}), 3.27$ (dd, $J=17.0,7.9 \mathrm{~Hz}, 1 \mathrm{H}) ;{ }^{13} \mathrm{C} \mathrm{NMR}\left(126 \mathrm{MHz}, \mathrm{CDCl}_{3}\right) \delta 199.0$ (s), 171.0 (s), 157.2 (s), 150.9 (s), 150.4 (s), 129.0 (s), 108.4 (s), 105.9 (s), 57.4 (s), 57.2 (s), 54.5 (s), 53.8 (s), 31.1 (s).

\section{methyl 5-fluoro-1-oxo-2,3-dihydro-1H-indene-2-carboxylate $21^{7}$}

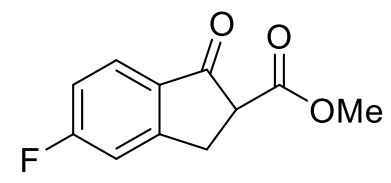

Following the Method A: Using 5-fluoro-2,3-dihydro-1H-inden-1-one instead of tetralone. Brown solid. Eluent: ethyl acetate/ petroleum ether (1:6). ${ }^{1} \mathrm{H} \mathrm{NMR}\left(500 \mathrm{MHz}, \mathrm{CDCl}_{3}\right)$ : The compound exists as a 8.3:1 mixture of keto/enol tautomer. Signals corresponding to the keto tautomer: $\delta 7.79(\mathrm{dd}, J=8.4$, $5.3 \mathrm{~Hz}, 1 \mathrm{H}), 7.17(\mathrm{~d}, J=8.0 \mathrm{~Hz}, 1 \mathrm{H}), 7.11(\mathrm{t}, J=8.4 \mathrm{~Hz}, 1 \mathrm{H}), 3.81(\mathrm{~s}, 3 \mathrm{H}), 3.77(\mathrm{dd}, J=8.3,4.0 \mathrm{~Hz}$, $1 \mathrm{H}), 3.57(\mathrm{dd}, J=17.5,3.7 \mathrm{~Hz}, 1 \mathrm{H}), 3.37$ (dd, $J=17.5,8.3 \mathrm{~Hz}, 1 \mathrm{H})$; Representative signals corresponding to the enol tautomer: $3.86(\mathrm{~s}, 3 \mathrm{H}), 3.52(\mathrm{~s}, 2 \mathrm{H}) ;{ }^{13} \mathrm{C}$ NMR $\left(126 \mathrm{MHz}, \mathrm{CDCl}_{3}\right)$ Signals corresponding to both tautomers: $\delta 198.5$ (s), 170.3 (s), $169.7(\mathrm{~s}), 167.7(\mathrm{~s}), 157.6(\mathrm{~d}, J=10.5 \mathrm{~Hz})$, 132.7 (s), 128.2 (d, $J=10.7 \mathrm{~Hz}), 117.5$ (s), 117.3 (s), 114.4 (s), 114.2 (s), 54.4 (s), 54.0 (s), 31.2 (d, $J$ $=2.2 \mathrm{~Hz})$. 
methyl 5-chloro-1-oxo-2,3-dihydro-1H-indene-2-carboxylate $2 \mathrm{~m}^{6}$<smiles>COC(=O)C1Cc2cc(Cl)ccc2C1=O</smiles>

Following the Method A: Using 5-chloro-2,3-dihydro-1H-inden-1-one instead of tetralone. Brown solid. Eluent: ethyl acetate/ petroleum ether (1:6). ${ }^{1} \mathrm{H} \mathrm{NMR}\left(500 \mathrm{MHz}, \mathrm{CDCl}_{3}\right)$ : The compound exists as a 3.5:1 mixture of keto/enol tautomer. Signals corresponding to the keto tautomer: ${ }^{1} \mathrm{H}$ NMR (500 $\left.\mathrm{MHz} \mathrm{CDCl}_{3}\right) \delta 7.70(\mathrm{~d}, J=8.2 \mathrm{~Hz}, 1 \mathrm{H}), 7.50(\mathrm{~s}, 1 \mathrm{H}), 7.38(\mathrm{~d}, J=7.7 \mathrm{~Hz}, 1 \mathrm{H}), 3.80(\mathrm{~s}, 3 \mathrm{H}), 3.76(\mathrm{dd}$, $J=8.3,4.0 \mathrm{~Hz}, 1 \mathrm{H}), 3.55(\mathrm{dd}, J=17.4,3.7 \mathrm{~Hz}, 1 \mathrm{H}), 3.35(\mathrm{dd}, J=17.4,8.3 \mathrm{~Hz}, 1 \mathrm{H})$; Representative

signals corresponding to the enol tautomer: 3.86 (s, 3H), $3.50(\mathrm{~s}, 2 \mathrm{H}) ;{ }^{13} \mathrm{C} \mathrm{NMR}\left(126 \mathrm{MHz}, \mathrm{CDCl}_{3}\right)$ Signals corresponding to both tautomers: $\delta 199.0$ (s), 170.2 (s), 156.1 (s), 145.8 (s), 143.2 (s), 134.8 (s), 129.8 (s), 128.5 (s), 127.9 (s), 126.9 (s), 126.3 (s), 122.8 (s), 54.3 (s), 54.0 (s), 52.4 (s), 33.5 (s), $31.1(\mathrm{~s})$.

methyl 5-bromo-1-oxo-2,3-dihydro-1H-indene-2-carboxylate $2 \mathbf{n}^{8}$<smiles>COC(=O)C1Cc2cc(Br)ccc2C1=O</smiles>

Following the Method A: Using 5-bromo-2,3-dihydro-1H-inden-1-one instead of tetralone. Brown solid. Eluent: ethyl acetate/ petroleum ether (1:6). ${ }^{1} \mathrm{H} \mathrm{NMR}\left(500 \mathrm{MHz}, \mathrm{CDCl}_{3}\right)$ : The compound exists as a 3.2:1 mixture of keto/enol tautomer. Signals corresponding to the keto tautomer: ${ }^{1} \mathrm{H}$ NMR (500 $\left.\mathrm{MHz}, \mathrm{CDCl}_{3}\right) \delta 7.70(\mathrm{~s}, 1 \mathrm{H}), 7.63(\mathrm{~s}, 1 \mathrm{H}), 7.53(\mathrm{dd}, J=15.0,6.9 \mathrm{~Hz}, 2 \mathrm{H}), 3.80(\mathrm{~s}, 3 \mathrm{H}), 3.75(\mathrm{dd}, J=$ 8.3, 4.0 Hz, 1H), 3.57 (dd, $J=17.5,3.9 \mathrm{~Hz}, 1 \mathrm{H}), 3.37$ (dd, $J=17.4,8.3 \mathrm{~Hz}, 1 \mathrm{H})$; Representative signals corresponding to the enol tautomer: $3.87(\mathrm{~s}, 3 \mathrm{H}), 3.51(\mathrm{~s}, 2 \mathrm{H}) ;{ }^{13} \mathrm{C}$ NMR $\left(126 \mathrm{MHz}, \mathrm{CDCl}_{3}\right)$ Signals corresponding to both tautomers: $\delta 199.2$ (s), 170.2 (s), 156.2 (s), 135.2 (s), 132.7 (s), 132.1 (s), 131.0 (s), 129.2 (s), 126.9 (s), 123.1 (s), 54.2 (s), 54.0 (s), 52.4 (s), 37.2 (s), 31.0 (s).

\section{methyl 5-oxo-6,7,8,9-tetrahydro-5H-benzo[7]annulene-6-carboxylate $2 \mathbf{u}^{1}$}<smiles>COC(=O)C1CCCc2ccccc2C1=O</smiles> 
Following the Method A: Using 1-benzosuberone instead of tetralone. Yellow oil. Eluent: ethyl acetate/ petroleum ether (1:6). ${ }^{1} \mathrm{H}$ NMR $\left(500 \mathrm{MHz}, \mathrm{CDCl}_{3}\right)$ : The compound exists as a 1:2.7 mixture of keto/enol tautomer. Signals corresponding to the enol tautomer: ${ }^{1} \mathrm{H}$ NMR $\left(500 \mathrm{MHz}, \mathrm{CDCl}_{3}\right) \delta 12.62$ (s, 1H), 7.63 (dd, J=7.2, 1.9 Hz, 1H), $7.36-7.31(\mathrm{~m}, 2 \mathrm{H}), 7.25-7.20(\mathrm{~m}, 1 \mathrm{H}), 3.84(\mathrm{~s}, 3 \mathrm{H}), 2.95$ (dd, $J=9.7,4.5 \mathrm{~Hz}, 1 \mathrm{H}), 2.64(\mathrm{t}, J=6.8 \mathrm{~Hz}, 2 \mathrm{H}), 2.16-2.08(\mathrm{~m}, 4 \mathrm{H})$; Representative signals corresponding to the keto tautomer: $7.76(\mathrm{dd}, J=7.7,1.1 \mathrm{~Hz}, 1 \mathrm{H}), 7.44(\mathrm{td}, J=7.5,1.2 \mathrm{~Hz}, 1 \mathrm{H}), 3.77(\mathrm{~s}, 3 \mathrm{H}) ;{ }^{13} \mathrm{C}$ NMR (126 MHz, $\mathrm{CDCl}_{3}$ ) Signals corresponding to both tautomers: $\delta 201.6$ (s), 174.5 (s), 171.9 (s), 171.5 (s), 142.4 (s), 142.2 (s), 139.0 (s), 136.8 (s), 133.6 (s), 131.2 (s), 131.0 (s), 130.3 (s), 130.1 (s), 128.2 (s), 127.9 (s), 127.4 (s), 101.3 (s), 57.6 (s), 53.4 (s), 52.8 (s), 34.6 (s), 33.9 (s), 32.8 (s), 26.4 (s), $25.4(\mathrm{~s}), 22.9(\mathrm{~s})$.

Method $\mathrm{B}^{9}$ :

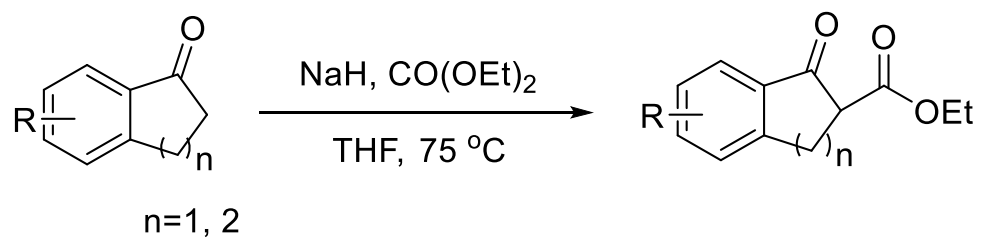

To a suspension of $\mathrm{NaH}$ (12.5 mmol, 2.5 equiv, $60 \%$ in mineral oil) and diethyl carbonate ( $8 \mathrm{mmol}, 2.0$ equiv) in THF ( $5 \mathrm{~mL})$ at room temperature was added a solution of tetralone (5 mmol, 1.0 equiv) in THF $(10 \mathrm{~mL})$ dropwise. The mixture was stirred at $75^{\circ} \mathrm{C}$ for $16 \mathrm{~h}$, then cooled to room temperature and acidified with $1 \mathrm{M}$ aq. $\mathrm{HCl}$, extracted with diethyl ether, dried over $\mathrm{MgSO}_{4}$ and concentrated. Purification by column chromatography (ethyl acetate/ petroleum ether $=1: 8$ ) gave the product $2 \mathrm{f}$ as a yellow oil.

\section{ethyl 1-oxo-1,2,3,4-tetrahydronaphthalene-2-carboxylate $2 f^{9}$}<smiles>CCOC(=O)C1CCc2ccccc2C1=O</smiles>

Yellow oil. Eluent: ethyl acetate/ petroleum ether (1:8). ${ }^{1} \mathrm{H}$ NMR (500 MHz, $\left.\mathrm{CDCl}_{3}\right)$ : The compound exists as a 1:1 mixture of keto/enol tautomer. Signals corresponding to the keto tautomer: ${ }^{1} \mathrm{H}$ NMR $\left(500 \mathrm{MHz}, \mathrm{CDCl}_{3}\right) 8.05$ (d, $\left.J=7.8 \mathrm{~Hz}, 1 \mathrm{H}\right), 7.49$ (td, $\left.J=7.5,1.1 \mathrm{~Hz}, 1 \mathrm{H}\right), 7.36-7.22(\mathrm{~m}, 2 \mathrm{H}), 4.31-$ $4.22(\mathrm{~m}, 2 \mathrm{H}), 3.60(\mathrm{dd}, J=10.5,4.7 \mathrm{~Hz}, 1 \mathrm{H}), 3.10-2.95(\mathrm{~m}, 2 \mathrm{H}), 2.54-2.45(\mathrm{~m}, 1 \mathrm{H}), 2.42-2.31(\mathrm{~m}$, 
1H), $1.29(\mathrm{t}, J=7.1 \mathrm{~Hz}, 3 \mathrm{H})$; Signals corresponding to the enol tautomer: $\delta 12.50(\mathrm{~s}, 1 \mathrm{H}), 7.80(\mathrm{~d}, J=$ $7.4 \mathrm{~Hz}, 1 \mathrm{H}), 7.36-7.22(\mathrm{~m}, 2 \mathrm{H}), 7.17(\mathrm{~d}, J=7.2 \mathrm{~Hz}, 1 \mathrm{H}), 4.31-4.22(\mathrm{~m}, 2 \mathrm{H}), 2.81$ (t, $J=7.7 \mathrm{~Hz}$, 2H), $2.57(\mathrm{t}, J=7.7 \mathrm{~Hz}, 2 \mathrm{H}), 1.35$ (t, $J=7.1 \mathrm{~Hz}, 3 \mathrm{H}) ;{ }^{13} \mathrm{C}$ NMR (126 MHz, CDCl 3 ) Signals corresponding to both tautomers: $\delta 194.4$ (s), 173.9 (s), 171.4 (s), 166.1 (s), 144.8 (s), 140.5 (s), 135.0 (s), 132.9 (s), 131.6 (s), 131.1 (s), 129.9 (s), 128.8 (s), 128.5 (s), 128.0 (s), 127.6 (s), 125.4 (s), 98.1 (s), $62.4(\mathrm{~s}), 61.6(\mathrm{~s}), 55.7(\mathrm{~s}), 28.9(\mathrm{~s}), 28.7(\mathrm{~s}), 27.5(\mathrm{~s}), 21.6(\mathrm{~s}), 15.4(\mathrm{~s}), 15.2(\mathrm{~s})$.

\section{ethyl 1-oxo-2,3-dihydro-1H-indene-2-carboxylate $20^{10}$}<smiles>CCOC(=O)C1Cc2ccccc2C1=O</smiles>

Following the Method B: Using indanone instead of tetralone. Yellow oil. Eluent: ethyl acetate/ petroleum ether (1:8). ${ }^{1} \mathrm{H}$ NMR $\left(500 \mathrm{MHz}, \mathrm{CDCl}_{3}\right)$ : The compound exists as a 5.7:1 mixture of keto/enol tautomer. Signals corresponding to the keto tautomer: ${ }^{1} \mathrm{H}$ NMR $\left(500 \mathrm{MHz}, \mathrm{CDCl}_{3}\right) \delta 7.74(\mathrm{~d}$, $J=7.7 \mathrm{~Hz}, 1 \mathrm{H}), 7.59(\mathrm{t}, J=7.4 \mathrm{~Hz}, 1 \mathrm{H}), 7.47(\mathrm{~d}, J=7.6 \mathrm{~Hz}, 1 \mathrm{H}), 7.36(\mathrm{t}, J=7.4 \mathrm{~Hz}, 1 \mathrm{H}), 4.22(\mathrm{q}, J=$ $7.1 \mathrm{~Hz}, 2 \mathrm{H}), 3.69(\mathrm{dd}, J=8.2,4.0 \mathrm{~Hz}, 1 \mathrm{H}), 3.53(\mathrm{dd}, J=17.2,3.8 \mathrm{~Hz}, 1 \mathrm{H}), 3.35(\mathrm{dd}, J=17.3,8.2 \mathrm{~Hz}$, 1H), 1.28 (t, $J=7.1 \mathrm{~Hz}, 3 \mathrm{H})$; Representative signals corresponding to the enol tautomer: 4.30 (q, $J=$ $7.1 \mathrm{~Hz}, 2 \mathrm{H}), 3.48(\mathrm{~s}, 2 \mathrm{H}), 1.34(\mathrm{t}, J=7.1 \mathrm{~Hz}, 3 \mathrm{H}) ;{ }^{13} \mathrm{C} \mathrm{NMR}\left(126 \mathrm{MHz}, \mathrm{CDCl}_{3}\right)$ Signals corresponding to both tautomers: $\delta 200.7$ (s), 170.2 (s), 154.7 (s), 144.3 (s), 138.0 (s), 136.5 (s), 136.4 (s), 130.4 (s), $128.9(\mathrm{~s}), 127.9$ (s), 127.6 (s), 125.8 (s), 125.6 (s), 121.7 (s), 103.6 (s), 62.8 (s), 61.2 (s), 54.4 (s), 33.6 (s), $31.4(\mathrm{~s}), 15.5(\mathrm{~s}), 15.3(\mathrm{~s})$.

Method $\mathrm{C}^{11}$ :

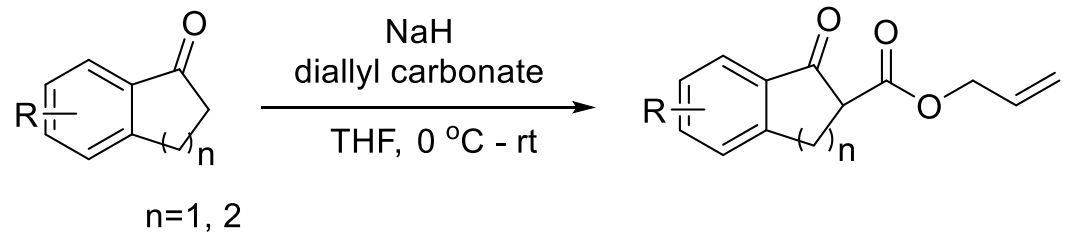

A flask was charged with $\mathrm{NaH}$ (12.5 mmol, 2.5 equiv, $60 \%$ dispersion in mineral oil). THF (10 mL) was added and the resultant solution cooled to $0{ }^{\circ} \mathrm{C}$ (ice/ $\mathrm{H}_{2} \mathrm{O}$ bath). A solution of 1-tetralone ( $5 \mathrm{mmol}$, 1.0 equiv) in THF (10 mL) was added dropwise. The solution was allowed to warm to room temperature. After 15 minutes, diallyl carbonate ( $7.5 \mathrm{mmol}, 1.5$ equiv.) was added dropwise. After $16 \mathrm{~h}$, the solution 
was quenched with saturated aqueous $\mathrm{NH}_{4} \mathrm{Cl}(20 \mathrm{~mL})$ and extracted with EtOAc $(3$ x $20 \mathrm{~mL})$. The combined organics were dried over anhydrous $\mathrm{MgSO}_{4}$, filtered, and concentrated in vacuo. The crude product was purified via silica gel column chromatography (ethyl acetate: petroleum ether $=1: 8$ ) to give the product $2 \mathrm{~g}$ as a yellow oil

\section{allyl 1-oxo-1,2,3,4-tetrahydronaphthalene-2-carboxylate $2 \mathrm{~g}^{12}$}<smiles>C=CCOC(=O)C1CCc2ccccc2C1=O</smiles>

Yellow oil. Eluent: ethyl acetate/ petroleum ether (1:8). ${ }^{1} \mathrm{H}$ NMR (500 MHz, $\mathrm{CDCl}_{3}$ ): The compound exists as a 1:1.1 mixture of keto/enol tautomer. Signals corresponding to the keto tautomer: ${ }^{1} \mathrm{H}$ NMR $\left(500 \mathrm{MHz} \mathrm{CDCl}_{3}\right) 8.06(\mathrm{~d}, J=7.8 \mathrm{~Hz}, 1 \mathrm{H}), 7.49$ (td, $\left.J=7.5,1.2 \mathrm{~Hz}, 1 \mathrm{H}\right), 7.34-7.24(\mathrm{~m}, 2 \mathrm{H}), 6.04-$ $5.91(\mathrm{~m}, 1 \mathrm{H}), 5.42-5.23(\mathrm{~m}, 2 \mathrm{H}), 4.76-4.63(\mathrm{~m}, 2 \mathrm{H}), 3.65(\mathrm{dd}, J=10.6,4.7 \mathrm{~Hz}, 1 \mathrm{H}), 3.11-2.96(\mathrm{~m}$, 2H), $2.52(\mathrm{dtd}, J=15.1,10.1,4.8 \mathrm{~Hz}, 1 \mathrm{H}), 2.38$ (ddd, $J=13.5,10.2,4.8 \mathrm{~Hz}, 1 \mathrm{H})$; Signals corresponding to the enol tautomer: $\delta 12.42(\mathrm{~s}, 1 \mathrm{H}), 7.81(\mathrm{~d}, J=7.5 \mathrm{~Hz}, 1 \mathrm{H}), 7.34-7.24(\mathrm{~m}, 2 \mathrm{H}), 7.18(\mathrm{~d}, J=7.3 \mathrm{~Hz}$, 1H), $6.04-5.91(\mathrm{~m}, 1 \mathrm{H}), 5.42-5.23(\mathrm{~m}, 2 \mathrm{H}), 4.76-4.63(\mathrm{~m}, 2 \mathrm{H}), 2.82(\mathrm{t}, J=7.7 \mathrm{~Hz}, 2 \mathrm{H}), 2.61(\mathrm{t}, J$ $=7.7 \mathrm{~Hz}, 2 \mathrm{H}),{ }^{13} \mathrm{C} \mathrm{NMR}\left(126 \mathrm{MHz}, \mathrm{CDCl}_{3}\right)$ Signals corresponding to both tautomers: $\delta 194.1(\mathrm{~s})$, 173.4 (s), 171.0 (s), 166.5 (s), 144.7 (s), 140.5 (s), 135.0 (s), 133.3 (s), 132.9 (s), 132.8 (s), 131.7 (s), 131.0 (s), 129.9 (s), 128.8 (s), 128.5 (s), 128.0 (s), 127.7 (s), 125.5 (s), 119.5 (s), 119.2 (s), 97.9 (s), $66.9(\mathrm{~s}), 66.2(\mathrm{~s}), 55.7(\mathrm{~s}), 28.9(\mathrm{~s}), 28.7(\mathrm{~s}), 27.5(\mathrm{~s}), 21.6(\mathrm{~s})$.

\section{allyl 1-oxo-2,3-dihydro-1H-indene-2-carboxylate $2 \mathbf{r}^{11}$}

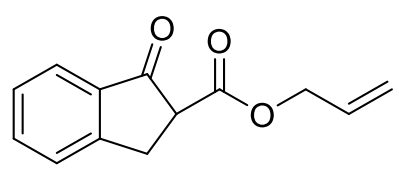

Following the Method C: Using indanone instead of tetralone. Brown oil. Eluent: ethyl acetate/ petroleum ether (1:8). ${ }^{1} \mathrm{H}$ NMR $\left(500 \mathrm{MHz}, \mathrm{CDCl}_{3}\right)$ : The compound exists as a 5.9:1 mixture of keto/enol tautomer. Signals corresponding to the keto tautomer: ${ }^{1} \mathrm{H}$ NMR $\left(500 \mathrm{MHz}, \mathrm{CDCl}_{3}\right) 7.74$ (d, $J=7.7 \mathrm{~Hz}, 1 \mathrm{H}), 7.60(\mathrm{dd}, J=13.9,6.6 \mathrm{~Hz}, 1 \mathrm{H}), 7.47(\mathrm{~d}, J=7.6 \mathrm{~Hz}, 1 \mathrm{H}), 7.36(\mathrm{t}, J=7.3 \mathrm{~Hz}, 1 \mathrm{H}), 5.96$ $-5.87(\mathrm{~m}, 1 \mathrm{H}), 5.39-5.19(\mathrm{~m}, 2 \mathrm{H}), 4.73-4.61(\mathrm{~m}, 2 \mathrm{H}), 3.77-3.71(\mathrm{~m}, 1 \mathrm{H}), 3.54(\mathrm{dd}, J=17.2,3.7$ $\mathrm{Hz}, 1 \mathrm{H}), 3.36$ (dd, $J=17.2,8.3 \mathrm{~Hz}, 1 \mathrm{H})$; Representative signals corresponding to the enol tautomer: $\delta$ 
$10.35(\mathrm{~s}, 1 \mathrm{H}), 6.05-5.97(\mathrm{~m}, 1 \mathrm{H}), 4.75(\mathrm{dd}, J=5.5,1.0 \mathrm{~Hz}, 2 \mathrm{H}), 3.50(\mathrm{~s}, 2 \mathrm{H}) ;{ }^{13} \mathrm{C} \mathrm{NMR}(126 \mathrm{MHz}$, $\mathrm{CDCl}_{3}$ ) Signals corresponding to both tautomers: $\delta 200.4(\mathrm{~s}), 169.9(\mathrm{~s}), 154.7(\mathrm{~s}), 144.3(\mathrm{~s}), 137.9(\mathrm{~s})$, 136.5 (s), 136.3 (s), 133.4 (s), 132.8 (s), 130.6 (s), 128.9 (s), 127.9 (s), 127.7 (s), 125.9 (s), 125.7 (s), $121.8(\mathrm{~s}), 119.7$ (s), $119.2(\mathrm{~s}), 103.2$ (s), 67.2 (s), 65.7 (s), 54.3 (s), 33.6 (s), $31.4(\mathrm{~s})$.

Method $\mathrm{D}^{10}$ :

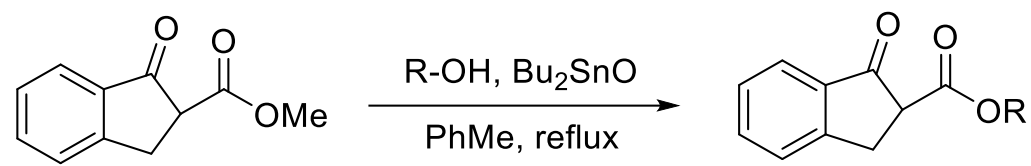

A solution of methyl 1-oxo-2,3-dihydro-1H-indene-2-carboxylate (2 mmol, 1.0 equiv), dibutyltin oxide (0.4 mmol, 0.2 equiv) and iso-propyl alcohol ( $6 \mathrm{mmol}, 3$ equiv) in toluene $(6 \mathrm{~mL})$ was refluxed for 16 h. After cooling to room temperature, the reaction mixture was poured into ice water and extracted with EtOAc (three times). The combined organic layer was washed with brine, dried over $\mathrm{MgSO}_{4}$, and concentrated in vacuo. The residue was purified by column chromatography on silica gel (ethyl acetate/ petroleum ether $=1: 8)$ to give the product $2 \mathrm{p}$ as a pale pink oil.

\section{iso-propyl 1-oxo-2,3-dihydro-1H-indene-2-carboxylate $2 \mathrm{p}^{10}$}<smiles>CC(C)OC(=O)C1Cc2ccccc2C1=O</smiles>

Pale pink oil. Eluent: ethyl acetate/ petroleum ether (1:8). ${ }^{1} \mathrm{H}$ NMR (500 MHz, $\left.\mathrm{CDCl}_{3}\right)$ : The compound exists as a 5.8:1 mixture of keto/enol tautomer. Signals corresponding to the keto tautomer: ${ }^{1} \mathrm{H}$ NMR $\left(500 \mathrm{MHz}, \mathrm{CDCl}_{3}\right) \delta 7.74(\mathrm{~d}, J=7.6 \mathrm{~Hz}, 1 \mathrm{H}), 7.59(\mathrm{t}, J=7.4 \mathrm{~Hz}, 1 \mathrm{H}), 7.48(\mathrm{~d}, J=7.6 \mathrm{~Hz}, 1 \mathrm{H}), 7.36$ (t, $J=7.5 \mathrm{~Hz}, 1 \mathrm{H}), 5.07(\mathrm{dt}, J=12.4,6.2 \mathrm{~Hz}, 1 \mathrm{H}), 3.66(\mathrm{dd}, J=8.2,4.0 \mathrm{~Hz}, 1 \mathrm{H}), 3.51(\mathrm{dd}, J=17.2$, $3.6 \mathrm{~Hz}, 1 \mathrm{H}), 3.34$ (dd, $J=17.2,8.2 \mathrm{~Hz}, 1 \mathrm{H}), 1.27$ (t, $J=6.0 \mathrm{~Hz}, 6 \mathrm{H})$; Representative signals corresponding to the enol tautomer: $5.19(\mathrm{dt}, J=12.4,6.2 \mathrm{~Hz}, 1 \mathrm{H}), 3.47(\mathrm{~s}, 2 \mathrm{H}), 1.32(\mathrm{~d}, J=6.2 \mathrm{~Hz}$, $6 \mathrm{H}) ;{ }^{13} \mathrm{C}$ NMR $\left(126 \mathrm{MHz}, \mathrm{CDCl}_{3}\right.$ ) Signals corresponding to both tautomers: $\delta 200.8$ (s), 169.8 (s), 154.7 (s), 144.3 (s), 138.1 (s), 136.5 (s), 136.4 (s), 130.3 (s), 128.8 (s), 127.8 (s), 127.6 (s), 125.8 (s), $125.6(\mathrm{~s}), 121.7$ (s), 103.9 (s), 70.3 (s), 68.6 (s), 54.6 (s), 33.7 (s), 31.4 (s), 23.2 (s), 22.9 (s), 22.8 (s). 
tert-butyl 1-oxo-2,3-dihydro-1H-indene-2-carboxylate $2 \mathrm{q}^{7}$

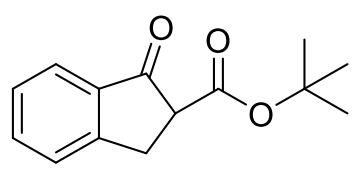

Following the Method D: Using tert-butyl alcohol instead of iso-propyl alcohol. Pink oil. Eluent: ethyl acetate/ petroleum ether (1:8). ${ }^{1} \mathrm{H}$ NMR $\left(500 \mathrm{MHz}, \mathrm{CDCl}_{3}\right)$ : The compound exists as a 8.5:1 mixture of keto/enol tautomer. Signals corresponding to the keto tautomer: ${ }^{1} \mathrm{H}$ NMR $\left(500 \mathrm{MHz}, \mathrm{CDCl}_{3}\right) \delta 7.77$ $(\mathrm{d}, J=7.6 \mathrm{~Hz}, 1 \mathrm{H}), 7.61(\mathrm{t}, J=7.4 \mathrm{~Hz}, 1 \mathrm{H}), 7.50(\mathrm{~d}, J=7.6 \mathrm{~Hz}, 1 \mathrm{H}), 7.39(\mathrm{t}, J=7.2 \mathrm{~Hz}, 1 \mathrm{H}), 3.63(\mathrm{dd}$, $J=8.1,3.9 \mathrm{~Hz}, 1 \mathrm{H}), 3.50(\mathrm{dd}, J=18.1,4.4 \mathrm{~Hz}, 1 \mathrm{H}), 3.34(\mathrm{dd}, J=17.1,8.1 \mathrm{~Hz}, 1 \mathrm{H}), 1.50(\mathrm{~s}, 9 \mathrm{H})$; Representative signals corresponding to the enol tautomer: 3.48 (s, 2H), $1.58(\mathrm{~s}, 9 \mathrm{H}) ;{ }^{13} \mathrm{C}$ NMR $(126$ $\left.\mathrm{MHz}, \mathrm{CDCl}_{3}\right) \delta 201.2(\mathrm{~s}), 169.4(\mathrm{~s}), 154.8$ (s), 136.3 (s), 128.8 (s), 127.6 (s), 125.7 (s), 83.2 (s), 55.5 (s), $31.4(\mathrm{~s}), 29.1(\mathrm{~s})$.

benzyl 1-oxo-2,3-dihydro-1H-indene-2-carboxylate $2 \mathrm{~s}^{13}$<smiles>O=C(OCc1ccccc1)C1Cc2ccccc2C1=O</smiles>

Following the Method D: Using benzyl alcohol instead of iso-propyl alcohol. Brown oil. Eluent: ethyl acetate/ petroleum ether (1:10). ${ }^{1} \mathrm{H} \mathrm{NMR}\left(500 \mathrm{MHz}_{\mathrm{CDCl}}\right)$ : The compound exists as a 6:1 mixture of keto/enol tautomer. Signals corresponding to the keto tautomer: ${ }^{1} \mathrm{H}$ NMR $\left(500 \mathrm{MHz}, \mathrm{CDCl}_{3}\right) \delta 7.79$ (d, $J=7.7 \mathrm{~Hz}, 1 \mathrm{H}), 7.62(\mathrm{t}, J=7.3 \mathrm{~Hz}, 1 \mathrm{H}), 7.50(\mathrm{~d}, J=7.6 \mathrm{~Hz}, 1 \mathrm{H}), 7.44-7.32(\mathrm{~m}, 6 \mathrm{H}), 5.24(\mathrm{~d}, J=4.0$ $\mathrm{Hz}, 2 \mathrm{H}), 3.79(\mathrm{dd}, J=8.3,4.1 \mathrm{~Hz}, 1 \mathrm{H}), 3.61-3.54(\mathrm{~m}, 1 \mathrm{H}), 3.38(\mathrm{dd}, J=17.2,8.3 \mathrm{~Hz}, 1 \mathrm{H})$; Representative signals corresponding to the enol tautomer: $5.33(\mathrm{~s}, 2 \mathrm{H}), 3.55(\mathrm{~s}, 2 \mathrm{H}) ;{ }^{13} \mathrm{C}$ NMR $(126$ $\mathrm{MHz}, \mathrm{CDCl}_{3}$ ) Signals corresponding to both tautomers: $\delta 200.4$ (s), 170.1 (s), 154.6 (s), 136.7 (s), 136.5 (s), 136.4 (s), 130.6 (s), 129.7 (s), 129.6 (s), 129.4 (s), 129.2 (s), 128.9 (s), 128.0 (s), 127.7 (s), 125.8 (s), 121.9 (s), $68.4(\mathrm{~s}), 66.8$ (s), 62.8 (s), 54.4 (s), 33.7 (s), 31.4 (s), 15.3 (s).

\section{adamantan-1-yl 1-oxo-2,3-dihydro-1H-indene-2-carboxylate $2 \mathrm{t}^{10}$}<smiles>COC(=O)C1Cc2ccccc2C1=O</smiles> 
Following the Method D: Using 1-adamantanol instead of iso-propyl alcohol. Pale pink solid. Eluent: ethyl acetate/ petroleum ether (1:8). ${ }^{1} \mathrm{H}$ NMR (500 MHz, $\mathrm{CDCl}_{3}$ ) Signals corresponding to the keto tautomer: $\delta 7.77(\mathrm{~d}, J=7.7 \mathrm{~Hz}, 1 \mathrm{H}), 7.61(\mathrm{dd}, J=10.7,4.2 \mathrm{~Hz}, 1 \mathrm{H}), 7.50(\mathrm{~d}, J=7.7 \mathrm{~Hz}, 1 \mathrm{H}), 7.39$ (t, $J=7.4 \mathrm{~Hz}, 1 \mathrm{H}), 3.63(\mathrm{dd}, J=8.2,4.0 \mathrm{~Hz}, 1 \mathrm{H}), 3.50(\mathrm{dd}, J=17.2,3.9 \mathrm{~Hz}, 1 \mathrm{H}), 3.34(\mathrm{dd}, J=17.2,8.2$ $\mathrm{Hz}, 1 \mathrm{H}), 2.17$ (s, 3H), 2.15 (s, 6H), $1.66(\mathrm{~s}, 6 \mathrm{H}) ;{ }^{13} \mathrm{C} \mathrm{NMR}\left(126 \mathrm{MHz}, \mathrm{CDCl}_{3}\right)$ Signals corresponding to both tautomers: $\delta 201.2$ (s), 169.1 (s), 154.8 (s), 136.6 (s), 136.3 (s), 128.7 (s), 127.6 (s), 125.7 (s), $83.3(\mathrm{~s}), 55.6(\mathrm{~s}), 46.4(\mathrm{~s}), 42.9(\mathrm{~s}), 42.3(\mathrm{~s}), 37.3(\mathrm{~s}), 37.2(\mathrm{~s}), 32.0(\mathrm{~s}), 31.8(\mathrm{~s}), 31.5(\mathrm{~s})$.

Method $\mathrm{E}^{14}$ :

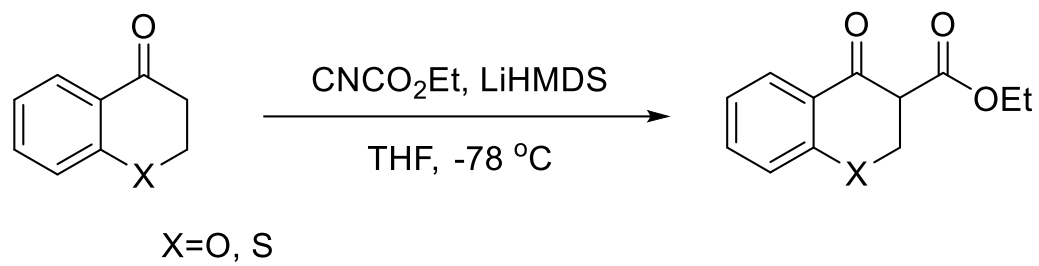

To a solution of 4-chromanone ( $5 \mathrm{mmol}, 1.0$ equiv) in THF $(10 \mathrm{~mL})$ stirred at $-78{ }^{\circ} \mathrm{C}$ was added a solution of LiHMDS (1 M in THF, $7.5 \mathrm{~mL}, 7.5 \mathrm{mmol}, 1.5$ equiv) dropwise and the mixture was stirred for 30 minutes. Ethyl cyanoformate $(10 \mathrm{mmol}, 2.0$ equiv) in THF $(10 \mathrm{~mL})$ was added dropwise and the reaction was stirred at $-78{ }^{\circ} \mathrm{C}$ for 1 hour. The mixture was allowed to warm to room temperature and was quenched by the addition of aq. $\mathrm{NH}_{4} \mathrm{Cl}(25 \mathrm{~mL})$ and $\mathrm{H}_{2} \mathrm{O}(25 \mathrm{~mL})$. The mixture was extracted with EtOAc $(3 \times 25 \mathrm{~mL})$. The combined organic phases were washed with brine $(30 \mathrm{~mL})$, dried $\left(\mathrm{MgSO}_{4}\right)$ and concentrated in vacuo. Flash column chromatography (ethyl acetate/ petroleum ether $=1: 15$ ) afforded the product $2 \mathrm{v}$ as a yellow oil.

ethyl 4-oxochromane-3-carboxylate $2 v^{14}$<smiles>CCOC(=O)C1COc2ccccc2C1=O</smiles>

Yellow oil. Eluent: ethyl acetate/ petroleum ether (1:15). ${ }^{1} \mathrm{H}$ NMR (500 MHz, $\left.\mathrm{CDCl}_{3}\right)$ : The compound exists as a 2:1 mixture of keto/enol tautomer. Signals corresponding to the keto tautomer: ${ }^{1} \mathrm{H}$ NMR $\left(500 \mathrm{MHz}, \mathrm{CDCl}_{3}\right) 7.88(\mathrm{dd}, J=7.8,1.6 \mathrm{~Hz}, 1 \mathrm{H}), 7.50-7.39(\mathrm{~m}, 1 \mathrm{H}), 7.00(\mathrm{t}, J=7.5 \mathrm{~Hz}, 1 \mathrm{H}), 6.94$ $(\mathrm{d}, J=8.2 \mathrm{~Hz}, 1 \mathrm{H}), 4.79-4.72(\mathrm{~m}, 1 \mathrm{H}), 4.61-4.54(\mathrm{~m}, 1 \mathrm{H}), 4.28-4.15(\mathrm{~m}, 2 \mathrm{H}), 3.74-3.66(\mathrm{~m}, 1 \mathrm{H})$, $1.24(\mathrm{dd}, J=8.4,5.8 \mathrm{~Hz}, 3 \mathrm{H})$; Signals corresponding to the enol tautomer: $\delta 12.00(\mathrm{~s}, 1 \mathrm{H}), 7.64-7.58$ 
(m, 1H), $7.31-7.23(\mathrm{~m}, 1 \mathrm{H}), 6.94(\mathrm{~d}, J=8.2 \mathrm{~Hz}, 1 \mathrm{H}), 6.82(\mathrm{~d}, J=8.2 \mathrm{~Hz}, 1 \mathrm{H}), 4.91(\mathrm{~d}, J=1.4 \mathrm{~Hz}$, $2 \mathrm{H}), 4.28-4.15(\mathrm{~m}, 2 \mathrm{H}), 1.30(\mathrm{t}, J=7.1 \mathrm{~Hz}, 3 \mathrm{H}),{ }^{13} \mathrm{C} \mathrm{NMR}\left(126 \mathrm{MHz}, \mathrm{CDCl}_{3}\right)$ Signals corresponding to both tautomers: $\delta 188.1(\mathrm{~s}), 170.8(\mathrm{~s}), 168.3(\mathrm{~s}), 163.7$ (s), $162.4(\mathrm{~s}), 158.7$ (s), 137.4 (s), 134.1 (s), $128.6(\mathrm{~s}), 125.5(\mathrm{~s}), 122.8(\mathrm{~s}), 122.5(\mathrm{~s}), 121.6(\mathrm{~s}), 119.4(\mathrm{~s}), 118.9(\mathrm{~s}), 117.8(\mathrm{~s}), 93.0$ (s), $69.2(\mathrm{~s}), 64.8$ (s), $62.9(\mathrm{~s}), 61.8(\mathrm{~s}), 53.6(\mathrm{~s}), 15.3(\mathrm{~s}), 15.1(\mathrm{~s})$.

\section{ethyl 4-oxothiochromane-3-carboxylate $2 \mathrm{w}^{15}$}<smiles>CCOC(=O)C1CSc2ccccc2C1=O</smiles>

Following the Method E: Using thiochroman-4-one instead of 4-chromanone. Yellow oil. Eluent: ethyl acetate/ petroleum ether (1:15). ${ }^{1} \mathrm{H} \mathrm{NMR}\left(500 \mathrm{MHz}_{\mathrm{CDCl}}\right)$ : The compound exists as a 1:2 mixture of keto/enol tautomer. Signals corresponding to the keto tautomer: ${ }^{1} \mathrm{H}$ NMR $\left(500 \mathrm{MHz}, \mathrm{CDCl}_{3}\right) 8.12$ (dd, $J=8.0,1.1 \mathrm{~Hz}, 1 \mathrm{H}), 7.38(\mathrm{td}, J=8.0,1.5 \mathrm{~Hz}, 1 \mathrm{H}), 7.28-7.24(\mathrm{~m}, 1 \mathrm{H}), 7.22-7.15(\mathrm{~m}, 1 \mathrm{H}), 4.33-$ $4.25(\mathrm{~m}, 2 \mathrm{H}), 3.88(\mathrm{dd}, J=10.1,3.6 \mathrm{~Hz}, 1 \mathrm{H}), 3.62(\mathrm{dd}, J=13.6,10.1 \mathrm{~Hz}, 1 \mathrm{H}), 3.32(\mathrm{dd}, J=13.6,3.6$ $\mathrm{Hz}, 1 \mathrm{H}), 1.28(\mathrm{t}, J=7.1 \mathrm{~Hz}, 3 \mathrm{H})$; Signals corresponding to the enol tautomer: $\delta 12.77(\mathrm{~s}, 1 \mathrm{H}), 7.87-$ $7.81(\mathrm{~m}, 1 \mathrm{H}), 7.28-7.24(\mathrm{~m}, 2 \mathrm{H}), 7.22-7.15(\mathrm{~m}, 1 \mathrm{H}), 4.33-4.25(\mathrm{~m}, 2 \mathrm{H}), 3.72(\mathrm{~s}, 2 \mathrm{H}), 1.35(\mathrm{t}, J=$ $7.1 \mathrm{~Hz}, 3 \mathrm{H}) ;{ }^{13} \mathrm{C} \mathrm{NMR}\left(126 \mathrm{MHz}, \mathrm{CDCl}_{3}\right)$ Signals corresponding to both tautomers: $\delta 190.9(\mathrm{~s}), 172.4$ (s), 169.8 (s), 166.8 (s), 142.6 (s), 138.2 (s), 134.7 (s), 132.0 (s), 131.4 (s), 130.8 (s), 130.3 (s), 128.5 (s), $128.4(\mathrm{~s}), 127.8(\mathrm{~s}), 126.6(\mathrm{~s}), 126.3$ (s), 94.9 (s), 62.9 (s), 62.3 (s), 55.1 (s), 29.9 (s), 24.4 (s), 15.4 (s), $15.2(\mathrm{~s})$.

Method $\mathrm{F}^{16}$ :<smiles>[CH2+]COC(=O)CC(=O)c1ccccc1</smiles>

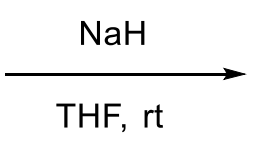<smiles>CCOC(=O)C(Cc1ccccc1)C(=O)c1ccccc1</smiles>

Ethyl benzoylacetate ( $5 \mathrm{mmol}, 1.0$ equiv) was added dropwise to a suspension of $\mathrm{NaH}$ ( $7.5 \mathrm{mmol}, 1.5$ equiv, 60\% dispersion in mineral oil) in THF $(10 \mathrm{~mL})$. The resulting mixture was stirred at room temperature until gas evolution finished and then alkyl bromide ( $5 \mathrm{mmol}, 1.0$ equiv) was added. The 
reaction mixture was stirred for $16 \mathrm{~h}$ at room temperature. The reaction was quenched with sat. aq. $\mathrm{NH} 4 \mathrm{Cl}$ and the aqueous layer was extracted with ethyl acetate $(3 \times 20 \mathrm{~mL})$. The combined organic extracts were dried over $\mathrm{MgSO}_{4}$, filtered and evaporated under reduced pressure. Product $2 \mathrm{y}$ was obtained by flash chromatography (ethyl acetate/ petroleum ether $=1: 10$ ) as a yellow oil.

ethyl 2-benzyl-3-oxo-3-phenylpropanoate $2 y^{16}$<smiles>CCOC(=O)C(Cc1ccccc1)C(=O)c1ccccc1</smiles>

${ }^{1} \mathrm{H} \mathrm{NMR}\left(500 \mathrm{MHz}, \mathrm{CDCl}_{3}\right) \delta 7.99(\mathrm{~d}, J=7.3 \mathrm{~Hz}, 2 \mathrm{H}), 7.56(\mathrm{t}, J=7.4 \mathrm{~Hz}, 1 \mathrm{H}), 7.45(\mathrm{t}, J=7.7 \mathrm{~Hz}$, 2H), $7.31-7.23(\mathrm{~m}, 4 \mathrm{H}), 7.20(\mathrm{td}, J=6.0,3.3 \mathrm{~Hz}, 1 \mathrm{H}), 4.67(\mathrm{t}, J=7.4 \mathrm{~Hz}, 1 \mathrm{H}), 4.15-4.06(\mathrm{~m}, 2 \mathrm{H})$, $3.42-3.30(\mathrm{~m}, 2 \mathrm{H}), 1.12(\mathrm{t}, J=7.1 \mathrm{~Hz}, 3 \mathrm{H}) ;{ }^{13} \mathrm{C} \mathrm{NMR}\left(126 \mathrm{MHz}, \mathrm{CDCl}_{3}\right) \delta 195.6(\mathrm{~s}), 170.4(\mathrm{~s}), 139.6$ (s), 137.3 (s), 134.7 (s), 130.1 (s), 129.8 (s), 129.8 (s), 129.6 (s), 127.8 (s), 62.6 (s), 57.3 (s), 35.9 (s), $15.0(\mathrm{~s})$.

$\mathbf{2 x}, \mathbf{2 z}$ and $2 \mathbf{a a}$ were purchased from commercial sources. 


\section{Table S1: Optimization of reaction conditions}

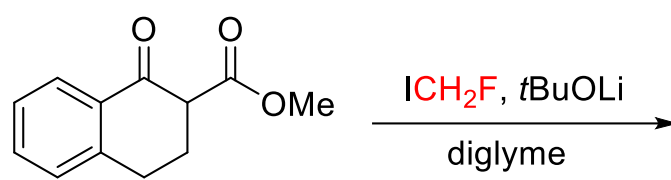

$2 \mathrm{a}$<smiles>COC(=O)C1=C(OCF)c2ccccc2CC1</smiles>

4a

\begin{tabular}{|c|c|c|c|c|c|c|c|}
\hline Entry & $\begin{array}{l}\mathrm{ICH}_{2} \mathrm{~F} \\
\text { (equiv) }\end{array}$ & $\begin{array}{l}t \mathrm{BuOLi} \\
\text { (equiv) }\end{array}$ & $\begin{array}{l}\mathrm{T} \\
\left({ }^{\circ} \mathrm{C}\right) \\
\end{array}$ & $\begin{array}{l}\text { diglyme } \\
(\mathrm{M})\end{array}$ & $\begin{array}{l}\text { Time } \\
(\mathrm{h})\end{array}$ & $\begin{array}{c}\text { Yield }^{\mathrm{a}} \\
(\%)\end{array}$ & $\mathrm{C} / \mathrm{O}$ ratio $^{\mathrm{b}}$ \\
\hline 1 & 1.5 & 2.0 & $\mathrm{rt}$ & 0.1 & 2 & 71 & $87: 13$ \\
\hline 2 & 2.0 & 2.0 & $\mathrm{rt}$ & 0.1 & 2 & 78 & $91: 9$ \\
\hline 3 & 2.5 & 2.0 & $\mathrm{rt}$ & 0.1 & 2 & 87 & $90: 10$ \\
\hline 4 & 3.0 & 2.0 & $\mathrm{rt}$ & 0.1 & 2 & 88 & $87: 13$ \\
\hline 5 & 2.5 & 1.5 & $\mathrm{rt}$ & 0.1 & 2 & 81 & $88: 12$ \\
\hline 6 & 2.5 & 2.5 & $\mathrm{rt}$ & 0.1 & 2 & 86 & $90: 10$ \\
\hline 7 & 2.5 & 3.0 & $\mathrm{rt}$ & 0.1 & 2 & 84 & $89: 11$ \\
\hline 8 & 2.5 & 2.0 & 0 & 0.1 & 2 & 34 & $92: 8$ \\
\hline 9 & 2.5 & 2.0 & 10 & 0.1 & 2 & 59 & $90: 10$ \\
\hline 10 & 2.5 & 2.0 & 40 & 0.1 & 2 & 91 & $81: 19$ \\
\hline 11 & 2.5 & 2.0 & $\mathrm{rt}$ & 0.05 & 2 & 86 & 89:11 \\
\hline 12 & 2.5 & 2.0 & $\mathrm{rt}$ & 0.15 & 2 & 79 & $86: 14$ \\
\hline 13 & 2.5 & 2.0 & $\mathrm{rt}$ & 0.2 & 2 & 77 & $85: 15$ \\
\hline 14 & 2.5 & 2.0 & $\mathrm{rt}$ & 0.1 & 0.5 & 55 & $90: 10$ \\
\hline 15 & 2.5 & 2.0 & $\mathrm{rt}$ & 0.1 & 4 & 86 & $89: 11$ \\
\hline 16 & 2.5 & 2.0 & $\mathrm{rt}$ & 0.1 & 8 & 86 & $87: 13$ \\
\hline 17 & 2.5 & 2.0 & $\mathrm{rt}$ & 0.1 & 24 & 85 & $87: 13$ \\
\hline
\end{tabular}

${ }^{a}$ Yields $(\mathbf{3 a}+\mathbf{4 a})$ were determined by GC; ${ }^{b}$ The ratio of $\mathbf{3 a} / \mathbf{4 a}$ was determined by ${ }^{19} \mathrm{~F}$ NMR spectroscopy with fluorobenzene as the internal standard.

After screening the equivalent of $\mathrm{ICH}_{2} \mathrm{~F}$ (entries 1-4), the equivalent of $t \mathrm{BuOLi}$ (entries 5-7), reaction temperature (entries 8-10), reaction concentration (entries 11-13), reaction time (entries 14-17), the combination of $\beta$-ketoesters $2 / \mathrm{ICH}_{2} \mathrm{~F}$ (2.5 equiv)/ $t \mathrm{BuOLi}(2.0$ equiv) in diglyme $(0.1 \mathrm{M})$ at room temperature for 2 hours, was selected as the optimized reaction condition. 


\section{General procedure for carbon-selective monofluoromethylation of $\beta$-ketoesters}

A mixture of $\beta$-ketoesters $2 \mathbf{a}-\mathbf{w}(0.2 \mathrm{mmol})$, fluoromethyl iodide $\left(\mathrm{ICH}_{2} \mathrm{~F}, 80 \mathrm{mg}, 0.5 \mathrm{mmol}\right), t \mathrm{BuOLi}$ (32 mg, $0.4 \mathrm{mmol}$ ) in $2.0 \mathrm{~mL}$ diglyme was stirred at room temperature for 2 hours. After completion, the reaction mixture was diluted with ethyl acetate, and then washed with water and brine. After drying over $\mathrm{Na}_{2} \mathrm{SO}_{4}$, the solvent was removed under reduced pressure, and the residue was subject to chromatography on silica gel to afford monofluoromethylated products 3a-w.

methyl 2-(fluoromethyl)-1-oxo-1,2,3,4-tetrahydronaphthalene-2-carboxylate 3a<smiles>COC(=O)C1(C)CCc2ccccc2C1=O</smiles>

Colorless oil, yield 75\% (35.4 mg). Eluent: ethyl acetate/ petroleum ether (1:40). ${ }^{1} \mathrm{H} \mathrm{NMR} \mathrm{(500} \mathrm{MHz,}$ $\left.\mathrm{CDCl}_{3}\right) \delta 8.06(\mathrm{~d}, J=7.9 \mathrm{~Hz}, 1 \mathrm{H}), 7.51(\mathrm{td}, J=7.6,1.2 \mathrm{~Hz}, 1 \mathrm{H}), 7.33(\mathrm{t}, J=7.5 \mathrm{~Hz}, 1 \mathrm{H}), 7.29-7.22$ (m, 1H), $4.96(\mathrm{dd}, J=46.6,9.1 \mathrm{~Hz}, 1 \mathrm{H}), 4.82(\mathrm{dd}, J=46.9,9.1 \mathrm{~Hz}, 1 \mathrm{H}), 3.71(\mathrm{~s}, 3 \mathrm{H}), 3.22-3.12$ (m, 1H), 3.00 (dt, $J=17.2,4.7 \mathrm{~Hz}, 1 \mathrm{H}), 2.63$ (dt, $J=13.8,4.7 \mathrm{~Hz}, 1 \mathrm{H}), 2.42$ (ddd, $J=13.8,11.1,4.8 \mathrm{~Hz}$, 1H); ${ }^{13} \mathrm{C}$ NMR (126 MHz, $\mathrm{CDCl}_{3}$ ) $\delta 191.4$ (s), 168.6 (s), 142.4 (s), 133.0 (s), 130.7 (s), 127.8 (s), 127.1 (s), 125.9 (s), 83.2 (d, $J=177.7 \mathrm{~Hz}), 57.5$ (d, $J=19.6 \mathrm{~Hz}), 51.8$ (s), 27.4 (s), 24.6 (s); ${ }^{19} \mathrm{~F}$ NMR (470 $\left.\mathrm{MHz}, \mathrm{CDCl}_{3}\right) \delta-230.1$ (t, $J=47.0 \mathrm{~Hz}$ ); HR-MS (EI) Calcd. For 236.0849, $\mathrm{C}_{13} \mathrm{H}_{13} \mathrm{FO}_{3}$, found 236.0841.

methyl 2-(fluoromethyl)-7-methyl-1-oxo-1,2,3,4-tetrahydronaphthalene-2-carboxylate 3b<smiles>COC(=O)C1(CF)CCc2ccc(C)cc2C1=O</smiles>

White solid, yield 77\% (38.5 mg). Eluent: ethyl acetate/ petroleum ether (1:40). ${ }^{1} \mathrm{H}$ NMR (500 MHz, $\left.\mathrm{CDCl}_{3}\right) \delta 7.86(\mathrm{~s}, 1 \mathrm{H}), 7.32(\mathrm{~d}, J=7.7 \mathrm{~Hz}, 1 \mathrm{H}), 7.14(\mathrm{~d}, J=7.8 \mathrm{~Hz}, 1 \mathrm{H}), 4.95(\mathrm{dd}, J=46.6,9.0 \mathrm{~Hz}$, $1 \mathrm{H}), 4.81(\mathrm{dd}, J=46.9,9.0 \mathrm{~Hz}, 1 \mathrm{H}), 3.70(\mathrm{~s}, 3 \mathrm{H}), 3.16-3.07(\mathrm{~m}, 1 \mathrm{H}), 2.95(\mathrm{dt}, J=17.1,4.7 \mathrm{~Hz}, 1 \mathrm{H})$, $2.61(\mathrm{dt}, J=13.7,4.7 \mathrm{~Hz}, 1 \mathrm{H}), 2.43-2.38(\mathrm{~m}, 1 \mathrm{H}), 2.36(\mathrm{~s}, 3 \mathrm{H}) ;{ }^{13} \mathrm{C} \mathrm{NMR}\left(126 \mathrm{MHz}, \mathrm{CDCl}_{3}\right) \delta 192.7$ (s), 169.8 (s), 140.6 (s), 136.7 (s), 135.1 (s), 131.5 (s), 128.8 (s), 128.2 (s), 84.3 (d, J = 177.7 Hz), 58.6 $(\mathrm{d}, J=19.7 \mathrm{~Hz}), 52.8(\mathrm{~s}), 28.6(\mathrm{~d}, J=4.1 \mathrm{~Hz}), 25.2(\mathrm{~s}), 21.0(\mathrm{~s}) ;{ }^{19} \mathrm{~F}$ NMR $\left(470 \mathrm{MHz}, \mathrm{CDCl}_{3}\right) \delta-229.9$ (t, $J=47.0 \mathrm{~Hz}$ ); HR-MS (EI) Calcd. For 250.1005, $\mathrm{C}_{14} \mathrm{H}_{15} \mathrm{FO}_{3}$, found 250.1011 . 
methyl 2-(fluoromethyl)-6-methoxy-1-oxo-1,2,3,4-tetrahydronaphthalene-2-carboxylate 3c<smiles>COCC12CC1(C(=O)OC)CCc1cc(OC)ccc1C2=O</smiles>

White solid, yield 79\% (42.0 mg). Eluent: ethyl acetate/ petroleum ether (1:40). ${ }^{1} \mathrm{H}$ NMR (500 MHz, $\left.\mathrm{CDCl}_{3}\right) \delta 8.01(\mathrm{~d}, J=8.8 \mathrm{~Hz}, 1 \mathrm{H}), 6.83(\mathrm{dd}, J=8.8,2.5 \mathrm{~Hz}, 1 \mathrm{H}), 6.68(\mathrm{~d}, J=2.3 \mathrm{~Hz}, 1 \mathrm{H}), 4.99-4.87$ $(\mathrm{m}, 1 \mathrm{H}), 4.87-4.75(\mathrm{~m}, 1 \mathrm{H}), 3.85(\mathrm{~s}, 3 \mathrm{H}), 3.70(\mathrm{~s}, 3 \mathrm{H}), 3.18-3.10(\mathrm{~m}, 1 \mathrm{H}), 2.94(\mathrm{dt}, J=17.2,4.8 \mathrm{~Hz}$, $1 \mathrm{H}), 2.60(\mathrm{dt}, J=13.7,4.8 \mathrm{~Hz}, 1 \mathrm{H}), 2.38(\mathrm{ddd}, J=13.8,10.9,4.8 \mathrm{~Hz}, 1 \mathrm{H}) ;{ }^{13} \mathrm{C} \mathrm{NMR}\left(126 \mathrm{MHz}, \mathrm{CDCl}_{3}\right)$ $\delta 190.8$ (s), 169.7 (d, $J=6.7 \mathrm{~Hz}), 164.0$ (s), 145.9 (s), 130.5 (s), 125.0 (s), 113.5 (s), 112.4 (s), 84.2 (d, $J=176.4 \mathrm{~Hz}), 58.1$ (d, $J=19.6 \mathrm{~Hz}), 55.3$ (s), 52.6 (s), 28.2 (d, $J=4.1 \mathrm{~Hz}), 25.8(\mathrm{~s}) ;{ }^{19} \mathrm{~F}$ NMR $(470$ $\left.\mathrm{MHz}, \mathrm{CDCl}_{3}\right) \delta-230.2\left(\mathrm{t}, J=47.0 \mathrm{~Hz}\right.$ ); HR-MS (EI) Calcd. For 266.0954, $\mathrm{C}_{14} \mathrm{H}_{15} \mathrm{FO}_{4}$, found 266.0950.

\section{methyl 2-(fluoromethyl)-7-methoxy-1-oxo-1,2,3,4-tetrahydronaphthalene-2-carboxylate 3d}<smiles>COC(=O)C1(C)CCc2ccc(OC)cc2C1=O</smiles>

White solid, yield 80\% (42.6 mg). Eluent: ethyl acetate/ petroleum ether (1:40). ${ }^{1} \mathrm{H}$ NMR (500 MHz, $\left.\mathrm{CDCl}_{3}\right) \delta 7.52(\mathrm{~d}, J=2.8 \mathrm{~Hz}, 1 \mathrm{H}), 7.16(\mathrm{~d}, J=8.4 \mathrm{~Hz}, 1 \mathrm{H}), 7.09(\mathrm{dd}, J=8.4,2.8 \mathrm{~Hz}, 1 \mathrm{H}), 4.96(\mathrm{dd}, J$ $=46.6,9.0 \mathrm{~Hz}, 1 \mathrm{H}), 4.80(\mathrm{dd}, J=46.9,9.0 \mathrm{~Hz}, 1 \mathrm{H}), 3.84(\mathrm{~s}, 3 \mathrm{H}), 3.71(\mathrm{~s}, 3 \mathrm{H}), 3.13-3.03(\mathrm{~m}, 1 \mathrm{H})$, $2.93(\mathrm{dt}, J=17.0,4.7 \mathrm{~Hz}, 1 \mathrm{H}), 2.60(\mathrm{dt}, J=13.7,4.6 \mathrm{~Hz}, 1 \mathrm{H}), 2.39$ (ddd, $J=13.8,11.1,4.9 \mathrm{~Hz}, 1 \mathrm{H})$; ${ }^{13} \mathrm{C} \mathrm{NMR}\left(126 \mathrm{MHz}, \mathrm{CDCl}_{3}\right) \delta 192.3$ (s), 169.6 (s), 158.3 (s), 135.9 (s), 132.2 (s), 129.9 (s), 122.5 (s), 109.6 (s), 84.0 (d, $J=177.7 \mathrm{~Hz}), 58.2$ (d, $J=19.5 \mathrm{~Hz}), 55.3$ (s), 52.7 (s), 28.5 (d, $J=4.1 \mathrm{~Hz}), 24.6$ (s); ${ }^{19} \mathrm{~F}$ NMR $\left(470 \mathrm{MHz}, \mathrm{CDCl}_{3}\right) \delta-229.6\left(\mathrm{t}, J=47.0 \mathrm{~Hz}\right.$ ); HR-MS (EI) Calcd. For 266.0954, $\mathrm{C}_{14} \mathrm{H}_{15} \mathrm{FO}_{4}$, found 266.0958 .

\section{methyl 7-bromo-2-(fluoromethyl)-1-oxo-1,2,3,4-tetrahydronaphthalene-2-carboxylate 3e}<smiles>COC(=O)C1(F)CCc2ccc(Br)cc2C1=O</smiles>

White solid, yield 68\% (42.7 mg). Eluent: ethyl acetate/ petroleum ether (1:40). ${ }^{1} \mathrm{H}$ NMR (500 MHz, $\left.\mathrm{CDCl}_{3}\right) \delta 8.17(\mathrm{~d}, J=2.1 \mathrm{~Hz}, 1 \mathrm{H}), 7.61(\mathrm{dd}, J=8.2,2.1 \mathrm{~Hz}, 1 \mathrm{H}), 7.15(\mathrm{~d}, J=8.2 \mathrm{~Hz}, 1 \mathrm{H}), 4.98(\mathrm{dd}, J$ $=46.5,9.1 \mathrm{~Hz}, 1 \mathrm{H}), 4.79(\mathrm{dd}, J=46.8,9.1 \mathrm{~Hz}, 1 \mathrm{H}), 3.71(\mathrm{~s}, 3 \mathrm{H}), 3.09(\mathrm{ddd}, J=16.5,11.3,4.7 \mathrm{~Hz}, 1 \mathrm{H})$, 
$2.95(\mathrm{dt}, J=17.3,4.5 \mathrm{~Hz}, 1 \mathrm{H}), 2.61(\mathrm{dt}, J=13.8,4.5 \mathrm{~Hz}, 1 \mathrm{H}), 2.40(\mathrm{ddd}, J=13.9,11.4,4.9 \mathrm{~Hz}, 1 \mathrm{H})$; ${ }^{13} \mathrm{C}$ NMR (126 MHz, $\left.\mathrm{CDCl}_{3}\right) \delta 191.1$ (s), 169.1 (d, $\left.J=7.3 \mathrm{~Hz}\right), 141.8$ (s), 136.6 (s), 133.0 (s), 130.7 (s), 130.5 (s), 120.8 (s), 83.9 (d, $J=176.4 \mathrm{~Hz}), 58.1$ (d, $J=19.8 \mathrm{~Hz}), 52.8$ (s), 29.5 (s), 28.1 (d, $J=4.1$ $\mathrm{Hz}), 25.0$ (s); ${ }^{19} \mathrm{~F} \mathrm{NMR}\left(470 \mathrm{MHz}, \mathrm{CDCl}_{3}\right) \delta$-230.2 (t, $J=47.0 \mathrm{~Hz}$ ); HR-MS (EI) Calcd. For 313.9954, $\mathrm{C}_{13} \mathrm{H}_{12} \mathrm{BrFO}_{3}$, found 313.9955 .

\section{ethyl 2-(fluoromethyl)-1-oxo-1,2,3,4-tetrahydronaphthalene-2-carboxylate 3f}

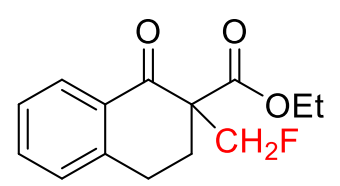

Colorless oil, yield 73\% (36.5 mg). Eluent: ethyl acetate/ petroleum ether (1:40). ${ }^{1} \mathrm{H}$ NMR (500 MHz, $\left.\mathrm{CDCl}_{3}\right) \delta 8.04(\mathrm{~d}, J=7.8 \mathrm{~Hz}, 1 \mathrm{H}), 7.48(\mathrm{t}, J=7.5 \mathrm{~Hz}, 1 \mathrm{H}), 7.31(\mathrm{t}, J=7.6 \mathrm{~Hz}, 1 \mathrm{H}), 7.23(\mathrm{~d}, J=7.7 \mathrm{~Hz}$, 1H), 4.95 (dd, $J=46.6,9.0 \mathrm{~Hz}, 1 \mathrm{H}), 4.80(\mathrm{dd}, J=46.9,9.0 \mathrm{~Hz}, 1 \mathrm{H}), 4.16(\mathrm{q}, J=7.1 \mathrm{~Hz}, 2 \mathrm{H}), 3.22-$ $3.12(\mathrm{~m}, 1 \mathrm{H}), 2.98(\mathrm{dt}, J=17.2,4.7 \mathrm{~Hz}, 1 \mathrm{H}), 2.61(\mathrm{dt}, J=13.7,4.7 \mathrm{~Hz}, 1 \mathrm{H}), 2.39$ (ddd, $J=13.8,11.1$, $4.9 \mathrm{~Hz}, 1 \mathrm{H}), 1.17$ (t, $J=7.1 \mathrm{~Hz}, 3 \mathrm{H}) ;{ }^{13} \mathrm{C} \mathrm{NMR}\left(126 \mathrm{MHz}, \mathrm{CDCl}_{3}\right) \delta 191.6$ (s), 168.2 (d, $\left.J=6.8 \mathrm{~Hz}\right)$, 142.4 (s), 133.0 (s), 130.8 (s), 127.9 (s), 127.1 (s), 125.9 (s), 83.2 (d, J=177.7 Hz), 60.9 (s), 57.5 (d, $J$ $=19.6 \mathrm{~Hz}), 27.4(\mathrm{~d}, J=4.1 \mathrm{~Hz}), 24.6(\mathrm{~s}), 13.0(\mathrm{~s}) ;{ }^{19} \mathrm{~F}$ NMR $\left(470 \mathrm{MHz}, \mathrm{CDCl}_{3}\right) \delta-229.2(\mathrm{t}, J=47.0$ $\mathrm{Hz}$ ); HR-MS (EI) Calcd. For 250.1005, $\mathrm{C}_{14} \mathrm{H}_{15} \mathrm{FO}_{3}$, found 250.1002.

\section{allyl 2-(fluoromethyl)-1-oxo-1,2,3,4-tetrahydronaphthalene-2-carboxylate $3 \mathrm{~g}$}

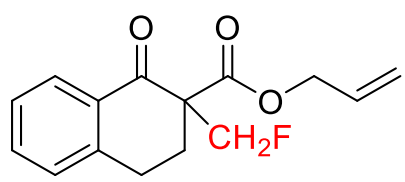

Colorless oil, yield 72\% (37.7 mg). Eluent: ethyl acetate/ petroleum ether (1:40). ${ }^{1} \mathrm{H} \mathrm{NMR} \mathrm{(500} \mathrm{MHz,}$ $\left.\mathrm{CDCl}_{3}\right) \delta 8.09(\mathrm{~d}, J=7.9 \mathrm{~Hz}, 1 \mathrm{H}), 7.54(\mathrm{t}, J=7.5 \mathrm{~Hz}, 1 \mathrm{H}), 7.36(\mathrm{t}, J=7.5 \mathrm{~Hz}, 1 \mathrm{H}), 7.28(\mathrm{~d}, J=7.9 \mathrm{~Hz}$, 1H), 5.83 (ddd, $J=15.8,11.0,5.5 \mathrm{~Hz}, 1 \mathrm{H}), 5.21(\mathrm{dd}, J=6.2,1.2 \mathrm{~Hz}, 1 \mathrm{H}), 5.18(\mathrm{~s}, 1 \mathrm{H}), 5.01(\mathrm{dd}, J=$ 46.6, 9.1 Hz, 1H), 4.87 (dd, $J=46.9,9.1 \mathrm{~Hz}, 1 \mathrm{H}), 4.70-4.60(\mathrm{~m}, 2 \mathrm{H}), 3.26-3.17(\mathrm{~m}, 1 \mathrm{H}), 3.04$ (dt, $J=17.2,4.7 \mathrm{~Hz}, 1 \mathrm{H}), 2.67(\mathrm{dt}, J=13.8,4.7 \mathrm{~Hz}, 1 \mathrm{H}), 2.46(\mathrm{ddd}, J=13.9,11.1,4.9 \mathrm{~Hz}, 1 \mathrm{H}) ;{ }^{13} \mathrm{C} \mathrm{NMR}$ $\left(126 \mathrm{MHz}, \mathrm{CDCl}_{3}\right) \delta 193.5$ (s), 170.0 (d, $\left.J=6.9 \mathrm{~Hz}\right), 144.4$ (s), 135.1 (s), 132.8 (s), 132.2 (s), 129.9 (s), 129.2 (s), 128.0 (s), 119.7 (s), 85.2 (d, $J=176.4 \mathrm{~Hz}), 67.2$ (s), 59.6 (d, $J=19.6 \mathrm{~Hz}), 29.4$ (d, $J=$ $4.2 \mathrm{~Hz}), 26.6(\mathrm{~s}) ;{ }^{19} \mathrm{~F}$ NMR $\left(470 \mathrm{MHz}, \mathrm{CDCl}_{3}\right) \delta-229.2(\mathrm{t}, J=47.0 \mathrm{~Hz})$; HR-MS (EI) Calcd. For 
262.1005, $\mathrm{C}_{15} \mathrm{H}_{15} \mathrm{FO}_{3}$, found 262.1013.

methyl 2-(fluoromethyl)-1-oxo-2,3-dihydro-1H-indene-2-carboxylate $3 \mathrm{~h}$

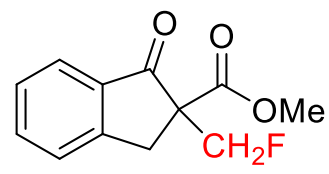

Colorless oil, yield 82\% (36.4 mg). Eluent: ethyl acetate/ petroleum ether (1:40). ${ }^{1} \mathrm{H} \mathrm{NMR} \mathrm{(500} \mathrm{MHz,}$ $\left.\mathrm{CDCl}_{3}\right) \delta 7.79(\mathrm{~d}, J=7.7 \mathrm{~Hz}, 1 \mathrm{H}), 7.66(\mathrm{t}, J=7.4 \mathrm{~Hz}, 1 \mathrm{H}), 7.53(\mathrm{~d}, J=7.7 \mathrm{~Hz}, 1 \mathrm{H}), 7.41(\mathrm{t}, J=7.5 \mathrm{~Hz}$, 1H), $4.94(\mathrm{~s}, 1 \mathrm{H}), 4.85(\mathrm{~s}, 1 \mathrm{H}), 3.72(\mathrm{~d}, J=17.3 \mathrm{~Hz}, 1 \mathrm{H}), 3.71(\mathrm{~s}, 3 \mathrm{H}), 3.43(\mathrm{~d}, J=17.3 \mathrm{~Hz}, 1 \mathrm{H}) ;{ }^{13} \mathrm{C}$ NMR (126 MHz, $\left.\mathrm{CDCl}_{3}\right) \delta 198.0$ (s), 167.8 (d, J=9.4 Hz), 152.3 (s), 134.8 (s), 133.9 (s), 127.0 (s), 125.5 (s), 124.0 (s), 82.7 (d, $J=173.9 \mathrm{~Hz}), 60.2$ (d, $J=21.4 \mathrm{~Hz}), 52.0$ (s), 33.2 (s); ${ }^{19} \mathrm{~F} \mathrm{NMR}(470 \mathrm{MHz}$, $\left.\mathrm{CDCl}_{3}\right) \delta-229.3\left(\mathrm{t}, J=47.0 \mathrm{~Hz}\right.$ ); HR-MS (EI) Calcd. For 222.0692, $\mathrm{C}_{12} \mathrm{H}_{11} \mathrm{FO}_{3}$, found 222.0697.

methyl 2-(fluoromethyl)-6-methyl-1-oxo-2,3-dihydro-1H-indene-2-carboxylate 3i

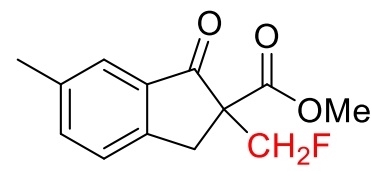

Colorless oil, yield 85\% (40.1 mg). Eluent: ethyl acetate/ petroleum ether (1:40). ${ }^{1} \mathrm{H} \mathrm{NMR} \mathrm{(500} \mathrm{MHz,}$ $\left.\mathrm{CDCl}_{3}\right) \delta 7.58(\mathrm{~s}, 1 \mathrm{H}), 7.47(\mathrm{~d}, J=7.8 \mathrm{~Hz}, 1 \mathrm{H}), 7.41(\mathrm{~d}, J=7.8 \mathrm{~Hz}, 1 \mathrm{H}), 4.93(\mathrm{~s}, 1 \mathrm{H}), 4.84(\mathrm{~s}, 1 \mathrm{H})$, $3.71(\mathrm{~s}, 3 \mathrm{H}), 3.66(\mathrm{~d}, J=16.4 \mathrm{~Hz}, 1 \mathrm{H}), 3.37(\mathrm{~d}, J=17.1 \mathrm{~Hz}, 1 \mathrm{H}), 2.41(\mathrm{~s}, 3 \mathrm{H}) ;{ }^{13} \mathrm{C} \mathrm{NMR}(126 \mathrm{MHz}$, $\left.\mathrm{CDCl}_{3}\right) \delta 198.0(\mathrm{~s}), 168.0(\mathrm{~s}), 149.8(\mathrm{~s}), 137.0(\mathrm{~s}), 136.1$ (s), 134.1 (s), $125.1(\mathrm{~s}), 123.8(\mathrm{~s}), 82.8(\mathrm{~d}, J=$ $175.1 \mathrm{~Hz}), 60.5$ (d, $J=20.2 \mathrm{~Hz}), 51.9$ (s), 32.9 (s), 20.0 (s); ${ }^{19} \mathrm{~F}$ NMR (470 MHz, $\mathrm{CDCl}_{3}$ ) $\delta-229.3$ (t, $J$ $=47.0 \mathrm{~Hz}$ ); HR-MS (EI) Calcd. For 236.0849, $\mathrm{C}_{13} \mathrm{H}_{13} \mathrm{FO}_{3}$, found 236.0840.

methyl 2-(fluoromethyl)-5-methoxy-1-oxo-2,3-dihydro-1H-indene-2-carboxylate 3j

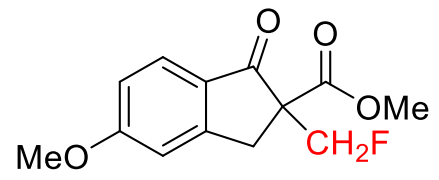

Colorless oil, yield 83\% (41.8 mg). Eluent: ethyl acetate/ petroleum ether (1:40). ${ }^{1} \mathrm{H} \mathrm{NMR} \mathrm{(500} \mathrm{MHz,}$ $\left.\mathrm{CDCl}_{3}\right) \delta 7.70(\mathrm{~d}, J=8.3 \mathrm{~Hz}, 1 \mathrm{H}), 6.95-6.91(\mathrm{~m}, 2 \mathrm{H}), 4.92(\mathrm{dd}, J=19.8,9.1 \mathrm{~Hz}, 1 \mathrm{H}), 4.82(\mathrm{q}, J=9.1$ $\mathrm{Hz}, 1 \mathrm{H}), 3.89$ (s, 3H), $3.71(\mathrm{~s}, 3 \mathrm{H}), 3.66$ (d, $J=17.3 \mathrm{~Hz}, 1 \mathrm{H}), 3.35(\mathrm{~d}, J=17.3 \mathrm{~Hz}, 1 \mathrm{H}) ;{ }^{13} \mathrm{C}$ NMR $(126$ $\left.\mathrm{MHz}, \mathrm{CDCl}_{3}\right) \delta 196.6(\mathrm{~d}, J=4.5 \mathrm{~Hz}), 168.8(\mathrm{~d}, J=9.3 \mathrm{~Hz}), 166.0(\mathrm{~s}), 156.3(\mathrm{~s}), 127.7$ (s), $126.4(\mathrm{~s})$, 
$116.0(\mathrm{~s}), 109.3(\mathrm{~s}), 83.6(\mathrm{~d}, J=173.9 \mathrm{~Hz}), 61.2(\mathrm{~d}, J=21.4 \mathrm{~Hz}), 55.5(\mathrm{~s}), 52.7$ (s), 33.9 (d, $J=3.5 \mathrm{~Hz})$; ${ }^{19} \mathrm{~F}$ NMR $\left(470 \mathrm{MHz}, \mathrm{CDCl}_{3}\right) \delta-229.4$ (t, $J=47.0 \mathrm{~Hz}$ ); HR-MS (EI) Calcd. For 252.0798, $\mathrm{C}_{13} \mathrm{H}_{13} \mathrm{FO}_{4}$, found 252.0804 .

methyl 2-(fluoromethyl)-5,6-dimethoxy-1-oxo-2,3-dihydro-1H-indene-2-carboxylate 3k<smiles>COC(=O)C1(C)Cc2cc(OC)c(OC)cc2C1=O</smiles>

Colorless oil, yield 86\% (48.5 mg). Eluent: ethyl acetate/ petroleum ether (1:40). ${ }^{1} \mathrm{H} \mathrm{NMR}(500 \mathrm{MHz}$, $\left.\mathrm{CDCl}_{3}\right) \delta 6.98(\mathrm{~s}, 1 \mathrm{H}), 6.75(\mathrm{~s}, 1 \mathrm{H}), 4.74(\mathrm{q}, J=9.1 \mathrm{~Hz}, 1 \mathrm{H}), 4.64(\mathrm{q}, J=9.1 \mathrm{~Hz}, 1 \mathrm{H}), 3.79(\mathrm{~s}, 3 \mathrm{H})$, $3.71(\mathrm{~s}, 3 \mathrm{H}), 3.53(\mathrm{~s}, 3 \mathrm{H}), 3.42(\mathrm{~d}, J=17.0 \mathrm{~Hz}, 1 \mathrm{H}), 3.13(\mathrm{~d}, J=17.0 \mathrm{~Hz}, 1 \mathrm{H}) ;{ }^{13} \mathrm{C} \mathrm{NMR}(126 \mathrm{MHz}$, $\left.\mathrm{CDCl}_{3}\right) \delta 196.3(\mathrm{~s}), 168.1$ (d, $\left.J=9.3 \mathrm{~Hz}\right), 155.5$ (s), 149.0 (s), 148.2 (s), 126.5 (s), 106.3 (s), 104.0 (s), $82.9(\mathrm{~d}, J=173.9 \mathrm{~Hz}), 60.6(\mathrm{~d}, J=21.4 \mathrm{~Hz}), 55.4(\mathrm{~s}), 55.1$ (s), $51.9(\mathrm{~s}), 33.0$ (d, $J=3.4 \mathrm{~Hz}) ;{ }^{19} \mathrm{~F}$ NMR $\left(470 \mathrm{MHz}, \mathrm{CDCl}_{3}\right) \delta-230.1\left(\mathrm{t}, J=47.0 \mathrm{~Hz}\right.$ ); HR-MS (EI) Calcd. For 282.0904, $\mathrm{C}_{14} \mathrm{H}_{15} \mathrm{FO}_{5}$, found 282.0905

methyl 5-fluoro-2-(fluoromethyl)-1-oxo-2,3-dihydro-1H-indene-2-carboxylate 31<smiles>COC(=O)C1(C)Cc2cc(F)ccc2C1=O</smiles>

Colorless oil, yield 78\% (37.4 mg). Eluent: ethyl acetate/ petroleum ether (1:40). ${ }^{1} \mathrm{H}$ NMR (500 MHz, $\left.\mathrm{CDCl}_{3}\right) \delta 7.82(\mathrm{dd}, J=8.5,5.3 \mathrm{~Hz}, 1 \mathrm{H}), 7.22(\mathrm{~d}, J=8.3 \mathrm{~Hz}, 1 \mathrm{H}), 7.14(\mathrm{td}, J=8.6,2.1 \mathrm{~Hz}, 1 \mathrm{H}), 4.97$ $(\mathrm{dd}, J=22.2,9.1 \mathrm{~Hz}, 1 \mathrm{H}), 4.87(\mathrm{dd}, J=22.7,9.1 \mathrm{~Hz}, 1 \mathrm{H}), 3.75(\mathrm{~s}, 3 \mathrm{H}), 3.73(\mathrm{~d}, J=17.6 \mathrm{~Hz}, 1 \mathrm{H}), 3.43$ $(\mathrm{d}, J=17.5 \mathrm{~Hz}, 1 \mathrm{H}) ;{ }^{13} \mathrm{C} \mathrm{NMR}\left(126 \mathrm{MHz}, \mathrm{CDCl}_{3}\right) \delta 198.3$ (s), 169.9 (s), 169.6 (d, $\left.J=9.9 \mathrm{~Hz}\right), 167.9$ (s), $157.4(\mathrm{~d}, J=10.6 \mathrm{~Hz}), 132.3$ (s), 128.5 (d, $J=10.7 \mathrm{~Hz}), 117.6(\mathrm{~d}, J=23.9 \mathrm{~Hz}), 114.4(\mathrm{~d}, J=22.7$ $\mathrm{Hz}), 84.7(\mathrm{~d}, J=173.9 \mathrm{~Hz}), 62.6(\mathrm{~d}, J=21.4 \mathrm{~Hz}), 54.2(\mathrm{~s}), 35.1(\mathrm{~d}, J=3.4 \mathrm{~Hz}) ;{ }^{19} \mathrm{~F}$ NMR $(470 \mathrm{MHz}$, $\left.\mathrm{CDCl}_{3}\right) \delta-99.6(\mathrm{~s}),-229.7\left(\mathrm{t}, J=47.0 \mathrm{~Hz}\right.$ ); HR-MS (EI) Calcd. For 240.0598, $\mathrm{C}_{12} \mathrm{H}_{10} \mathrm{~F}_{2} \mathrm{O}_{3}$, found 240.0602.

methyl 5-chloro-2-(fluoromethyl)-1-oxo-2,3-dihydro-1H-indene-2-carboxylate 3m 


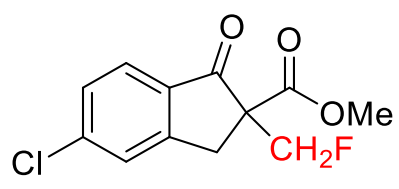

White solid, yield 73\% (37.4 mg). Eluent: ethyl acetate/ petroleum ether (1:40). ${ }^{1} \mathrm{H}$ NMR (500 MHz, $\left.\mathrm{CDCl}_{3}\right) \delta 7.72(\mathrm{~d}, J=8.2 \mathrm{~Hz}, 1 \mathrm{H}), 7.53(\mathrm{~s}, 1 \mathrm{H}), 7.40(\mathrm{dd}, J=8.2,0.8 \mathrm{~Hz}, 1 \mathrm{H}), 4.94(\mathrm{dd}, J=26.2,9.2$ Hz, 1H), 4.85 (dd, $J=26.6,9.2 \mathrm{~Hz}, 1 \mathrm{H}), 3.72$ (s, 3H), 3.69 (d, $J=17.5 \mathrm{~Hz}, 1 \mathrm{H}), 3.41$ (d, $J=17.5 \mathrm{~Hz}$, 1H); ${ }^{13} \mathrm{C}$ NMR (126 MHz, $\left.\mathrm{CDCl}_{3}\right) \delta 196.6$ (s), 167.4 (d, J=9.5 Hz), 153.7 (s), 141.6 (s), 132.3 (s), 127.9 (s), 125.7 (s), 125.0 (s), 82.5 (d, $J=175.1 \mathrm{~Hz}), 60.3$ (d, $J=20.5 \mathrm{~Hz}), 52.1(\mathrm{~s}), 32.9$ (s); ${ }^{19} \mathrm{~F} \mathrm{NMR}$ $\left(470 \mathrm{MHz}, \mathrm{CDCl}_{3}\right) \delta-230.2\left(\mathrm{t}, J=47.0 \mathrm{~Hz}\right.$ ); HR-MS (EI) Calcd. For 256.0303, $\mathrm{C}_{12} \mathrm{H}_{10} \mathrm{ClFO}_{3}$, found 256.0311.

methyl 5-bromo-2-(fluoromethyl)-1-oxo-2,3-dihydro-1H-indene-2-carboxylate 3n<smiles>COC(=O)C1(C)Cc2cc(Br)ccc2C1=O</smiles>

White solid, yield 69\% (41.4 mg). Eluent: ethyl acetate/ petroleum ether (1:40). ${ }^{1} \mathrm{H}$ NMR (500 MHz, $\left.\mathrm{CDCl}_{3}\right) \delta 7.71(\mathrm{~s}, 1 \mathrm{H}), 7.63(\mathrm{~d}, J=8.2 \mathrm{~Hz}, 1 \mathrm{H}), 7.55(\mathrm{dd}, J=8.2,0.7 \mathrm{~Hz}, 1 \mathrm{H}), 4.93(\mathrm{dd}, J=27.1,9.2$ $\mathrm{Hz}, 1 \mathrm{H}), 4.84$ (dd, $J=27.5,9.2 \mathrm{~Hz}, 1 \mathrm{H}), 3.71(\mathrm{~s}, 3 \mathrm{H}), 3.69$ (d, $J=17.5 \mathrm{~Hz}, 1 \mathrm{H}), 3.40$ (d, $J=17.5 \mathrm{~Hz}$, 1H); ${ }^{13} \mathrm{C}$ NMR (126 MHz, $\mathrm{CDCl}_{3}$ ) $\delta 196.9$ (s), 167.4 (s), 153.9 (s), 132.8 (s), 130.8 (s), 130.5 (s), 128.9 (s), $125.1(\mathrm{~s}), 82.5$ (d, $J=175.1 \mathrm{~Hz}), 60.3$ (d, $J=21.4 \mathrm{~Hz}), 52.1(\mathrm{~s}), 32.9$ (d, $J=3.6 \mathrm{~Hz}) ;{ }^{19} \mathrm{~F} \mathrm{NMR}$ $\left(470 \mathrm{MHz}, \mathrm{CDCl}_{3}\right) \delta-230.2\left(\mathrm{t}, J=47.0 \mathrm{~Hz}\right.$ ); HR-MS (EI) Calcd. For 299.9797, $\mathrm{C}_{12} \mathrm{H}_{10} \mathrm{BrFO}_{3}$, found 299.9799 .

\section{ethyl 2-(fluoromethyl)-1-oxo-2,3-dihydro-1H-indene-2-carboxylate 30}

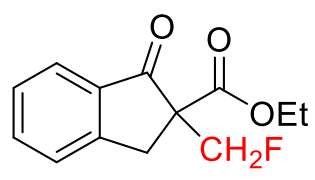

Colorless oil, yield 80\% (37.8 mg). Eluent: ethyl acetate/ petroleum ether (1:40). ${ }^{1} \mathrm{H} \mathrm{NMR} \mathrm{(500} \mathrm{MHz,}$ $\left.\mathrm{CDCl}_{3}\right) \delta 7.78(\mathrm{~d}, J=7.7 \mathrm{~Hz}, 1 \mathrm{H}), 7.64(\mathrm{dd}, J=10.9,4.0 \mathrm{~Hz}, 1 \mathrm{H}), 7.52(\mathrm{~d}, J=7.7 \mathrm{~Hz}, 1 \mathrm{H}), 7.40(\mathrm{t}, J$ $=7.5 \mathrm{~Hz}, 1 \mathrm{H}), 4.94(\mathrm{~s}, 1 \mathrm{H}), 4.84(\mathrm{~s}, 1 \mathrm{H}), 4.17(\mathrm{q}, J=7.1 \mathrm{~Hz}, 2 \mathrm{H}), 3.70(\mathrm{~d}, J=17.3 \mathrm{~Hz}, 1 \mathrm{H}), 3.41(\mathrm{~d}, J$ $=17.3 \mathrm{~Hz}, 1 \mathrm{H}), 1.21(\mathrm{t}, J=7.1 \mathrm{~Hz}, 3 \mathrm{H}) ;{ }^{13} \mathrm{C} \mathrm{NMR}\left(126 \mathrm{MHz}, \mathrm{CDCl}_{3}\right) \delta 200.2(\mathrm{~s}), 169.3(\mathrm{~d}, J=9.5$ 
Hz), 154.4 (s), 136.8 (s), 135.9 (s), 128.9 (s), 127.5 (s), 125.9 (s), 84.8 (d, J=173.9 Hz), 63.1 (s), 62.3 $(\mathrm{d}, J=21.2 \mathrm{~Hz}), 35.3(\mathrm{~d}, J=3.5 \mathrm{~Hz}), 15.0(\mathrm{~s}) ;{ }^{19} \mathrm{~F} \mathrm{NMR}\left(470 \mathrm{MHz}, \mathrm{CDCl}_{3}\right) \delta-230.1(\mathrm{t}, J=47.0 \mathrm{~Hz})$; HR-MS (EI) Calcd. For 236.0849, $\mathrm{C}_{13} \mathrm{H}_{13} \mathrm{FO}_{3}$, found 236.0855.

\section{isopropyl 2-(fluoromethyl)-1-oxo-2,3-dihydro-1H-indene-2-carboxylate 3p}

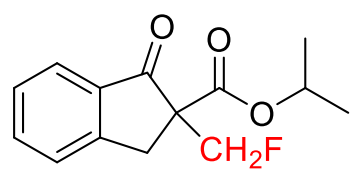

Colorless oil, yield 81\% (40.5 mg). Eluent: ethyl acetate/ petroleum ether (1:40). ${ }^{1} \mathrm{H} \mathrm{NMR}(500 \mathrm{MHz}$, $\left.\mathrm{CDCl}_{3}\right) \delta 7.76(\mathrm{~d}, J=7.7 \mathrm{~Hz}, 1 \mathrm{H}), 7.63(\mathrm{t}, J=7.4 \mathrm{~Hz}, 1 \mathrm{H}), 7.52(\mathrm{~d}, J=7.7 \mathrm{~Hz}, 1 \mathrm{H}), 7.39(\mathrm{t}, J=7.4 \mathrm{~Hz}$, 1H), $5.02(\mathrm{dt}, J=12.5,6.2 \mathrm{~Hz}, 1 \mathrm{H}), 4.95-4.89(\mathrm{~m}, 1 \mathrm{H}), 4.86-4.80(\mathrm{~m}, 1 \mathrm{H}), 3.68(\mathrm{~d}, J=17.3 \mathrm{~Hz}$, $1 \mathrm{H}), 3.39(\mathrm{~d}, J=17.2 \mathrm{~Hz}, 1 \mathrm{H}), 1.18(\mathrm{dd}, J=10.7,6.3 \mathrm{~Hz}, 6 \mathrm{H}) ;{ }^{13} \mathrm{C} \mathrm{NMR}\left(126 \mathrm{MHz}, \mathrm{CDCl}_{3}\right) \delta 199.2$ (s), 167.7 (d, $J=9.6 \mathrm{~Hz}), 153.3$ (s), 135.5 (s), 134.8 (s), 127.7 (s), 126.3 (s), 124.7 (s), 83.6 (d, $J=$ $173.9 \mathrm{~Hz}), 69.6(\mathrm{~s}), 61.2(\mathrm{~d}, J=20.2 \mathrm{~Hz}), 34.1(\mathrm{~d}, J=3.5 \mathrm{~Hz}), 21.3(\mathrm{~d}, J=8.5 \mathrm{~Hz}) ;{ }^{19} \mathrm{~F}$ NMR $(470$ $\left.\mathrm{MHz}, \mathrm{CDCl}_{3}\right) \delta-230.4\left(\mathrm{t}, J=47.0 \mathrm{~Hz}\right.$ ); HR-MS (EI) Calcd. For 250.1005, $\mathrm{C}_{14} \mathrm{H}_{15} \mathrm{FO}_{3}$, found 250.1010.

\section{tert-butyl 2-(fluoromethyl)-1-oxo-2,3-dihydro-1H-indene-2-carboxylate 3q}

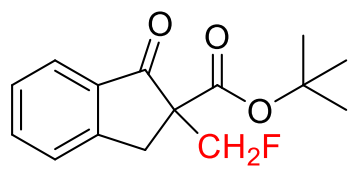

Colorless oil, yield 78\% (41.2 mg). Eluent: ethyl acetate/ petroleum ether (1:40). ${ }^{1} \mathrm{H}$ NMR (500 MHz, $\left.\mathrm{CDCl}_{3}\right) \delta 7.76(\mathrm{~d}, J=7.7 \mathrm{~Hz}, 1 \mathrm{H}), 7.62(\mathrm{t}, J=7.4 \mathrm{~Hz}, 1 \mathrm{H}), 7.51(\mathrm{~d}, J=7.7 \mathrm{~Hz}, 1 \mathrm{H}), 7.38(\mathrm{t}, J=7.4 \mathrm{~Hz}$, 1H), $4.90(\mathrm{dd}, J=28.5,9.1 \mathrm{~Hz}, 1 \mathrm{H}), 4.80(\mathrm{dd}, J=28.9,9.1 \mathrm{~Hz}, 1 \mathrm{H}), 3.64(\mathrm{~d}, J=17.2 \mathrm{~Hz}, 1 \mathrm{H}), 3.38(\mathrm{~d}$, $J=17.2 \mathrm{~Hz}, 1 \mathrm{H}), 1.38(\mathrm{~s}, 9 \mathrm{H}) ;{ }^{13} \mathrm{C} \mathrm{NMR}\left(126 \mathrm{MHz} \mathrm{CDCl}_{3}\right) \delta 199.5(\mathrm{~s}), 167.2(\mathrm{~d}, J=9.9 \mathrm{~Hz}), 153.3$ (s), 135.4 (s), 134.9 (s), 127.6 (s), 126.2 (s), 124.6 (s), 83.6 (d, J=173.9 Hz), 82.7 (s), 61.8 (d, J=21.4 $\mathrm{Hz}), 34.1(\mathrm{~d}, J=3.5 \mathrm{~Hz}), 27.6(\mathrm{~s}) ;{ }^{19} \mathrm{~F} \mathrm{NMR}\left(470 \mathrm{MHz}, \mathrm{CDCl}_{3}\right) \delta-230.6(\mathrm{t}, J=47.0 \mathrm{~Hz})$; HR-MS (EI) Calcd. For 264.1162, $\mathrm{C}_{15} \mathrm{H}_{17} \mathrm{FO}_{3}$, found 264.1165.

allyl 2-(fluoromethyl)-1-oxo-2,3-dihydro-1H-indene-2-carboxylate 3r

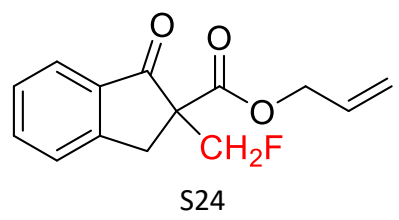


Colorless oil, yield 81\% (40.2 mg). Eluent: ethyl acetate/ petroleum ether (1:40). ${ }^{1} \mathrm{H} \mathrm{NMR} \mathrm{(500} \mathrm{MHz,}$ $\left.\mathrm{CDCl}_{3}\right) \delta 7.79(\mathrm{~d}, J=7.7 \mathrm{~Hz}, 1 \mathrm{H}), 7.66(\mathrm{t}, J=7.4 \mathrm{~Hz}, 1 \mathrm{H}), 7.54(\mathrm{~d}, J=7.7 \mathrm{~Hz}, 1 \mathrm{H}), 7.42(\mathrm{t}, J=7.5 \mathrm{~Hz}$, 1H), 5.83 (ddd, $J=22.6,10.8,5.5 \mathrm{~Hz}, 1 \mathrm{H}), 5.28-5.17(\mathrm{~m}, 2 \mathrm{H}), 5.00-4.93(\mathrm{~m}, 1 \mathrm{H}), 4.89-4.84$ (m, 1H), $4.62(\mathrm{~d}, J=5.5 \mathrm{~Hz}, 2 \mathrm{H}), 3.73(\mathrm{~d}, J=17.3 \mathrm{~Hz}, 1 \mathrm{H}), 3.44(\mathrm{~d}, J=17.3 \mathrm{~Hz}, 1 \mathrm{H}) ;{ }^{13} \mathrm{C} \mathrm{NMR}(126 \mathrm{MHz}$, $\left.\mathrm{CDCl}_{3}\right) \delta 198.8(\mathrm{~s}), 167.8(\mathrm{~d}, J=9.4 \mathrm{~Hz}), 153.2(\mathrm{~s}), 135.7$ (s), 134.7 (s), $131.0(\mathrm{~s}), 127.8$ (s), 126.3 (s), 124.8 (s), 118.5 (s), 83.6 (d, $J=173.9 \mathrm{~Hz}), 66.1(\mathrm{~s}), 61.1(\mathrm{~d}, J=20.9 \mathrm{~Hz}), 34.1(\mathrm{~d}, J=3.5 \mathrm{~Hz}) ;{ }^{19} \mathrm{~F}$ NMR (470 MHz, $\left.\mathrm{CDCl}_{3}\right) \delta-230.1\left(\mathrm{t}, J=47.0 \mathrm{~Hz}\right.$ ); HR-MS (EI) Calcd. For 248.0849, $\mathrm{C}_{14} \mathrm{H}_{13} \mathrm{FO}_{3}$, found 248.0854 .

\section{benzyl 2-(fluoromethyl)-1-oxo-2,3-dihydro-1H-indene-2-carboxylate 3s}

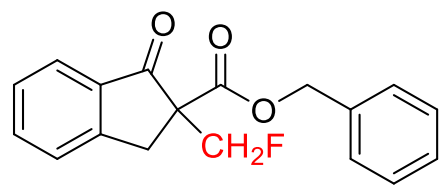

Colorless oil, yield 74\% (44.1 mg). Eluent: ethyl acetate/ petroleum ether (1:40). ${ }^{1} \mathrm{H}$ NMR (500 MHz, $\left.\mathrm{CDCl}_{3}\right) \delta 7.79(\mathrm{~d}, J=7.7 \mathrm{~Hz}, 1 \mathrm{H}), 7.65(\mathrm{t}, J=7.4 \mathrm{~Hz}, 1 \mathrm{H}), 7.52(\mathrm{~d}, J=7.7 \mathrm{~Hz}, 1 \mathrm{H}), 7.41(\mathrm{t}, J=7.5 \mathrm{~Hz}$, 1H), $7.35-7.29(\mathrm{~m}, 3 \mathrm{H}), 7.27-7.22(\mathrm{~m}, 2 \mathrm{H}), 5.16(\mathrm{q}, J=12.5 \mathrm{~Hz}, 2 \mathrm{H}), 4.96(\mathrm{~s}, 1 \mathrm{H}), 4.87(\mathrm{~s}, 1 \mathrm{H})$, $3.72(\mathrm{~d}, J=17.3 \mathrm{~Hz}, 1 \mathrm{H}), 3.43(\mathrm{~d}, J=17.3 \mathrm{~Hz}, 1 \mathrm{H}) ;{ }^{13} \mathrm{C} \mathrm{NMR}\left(126 \mathrm{MHz}, \mathrm{CDCl}_{3}\right) \delta 198.0(\mathrm{~s}), 167.2$ (d, $J=9.2 \mathrm{~Hz}), 152.4$ (s), 134.9 (s), 134.2 (s), 133.9 (s), 127.6 (s), 127.4 (s), 127.0 (s), 126.8 (s), 125.6 (s), $124.1(\mathrm{~s}), 82.8(\mathrm{~d}, J=175.1 \mathrm{~Hz}), 66.5(\mathrm{~s}), 60.4(\mathrm{~d}, J=21.4 \mathrm{~Hz}), 33.3(\mathrm{~d}, J=3.4 \mathrm{~Hz}) ;{ }^{19} \mathrm{~F}$ NMR $(470$ $\left.\mathrm{MHz}, \mathrm{CDCl}_{3}\right) \delta-230.9\left(\mathrm{t}, J=47.0 \mathrm{~Hz}\right.$ ); HR-MS (EI) Calcd. For 298.1005, $\mathrm{C}_{18} \mathrm{H}_{15} \mathrm{FO}_{3}$, found 298.1008.

\section{adamantan-1-yl 2-(fluoromethyl)-1-oxo-2,3-dihydro-1H-indene-2-carboxylate 3t}

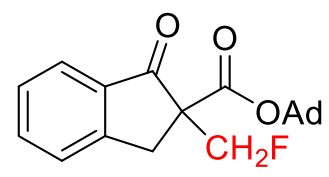

Colorless oil, yield 83\% (56.7 mg). Eluent: ethyl acetate/ petroleum ether (1:40). ${ }^{1} \mathrm{H}$ NMR (500 MHz, $\left.\mathrm{CDCl}_{3}\right) \delta 7.78(\mathrm{~d}, J=7.7 \mathrm{~Hz}, 1 \mathrm{H}), 7.63(\mathrm{t}, J=7.4 \mathrm{~Hz}, 1 \mathrm{H}), 7.51(\mathrm{~d}, J=7.7 \mathrm{~Hz}, 1 \mathrm{H}), 7.40(\mathrm{t}, J=7.4 \mathrm{~Hz}$, $1 \mathrm{H}), 4.91(\mathrm{dd}, J=35.0,9.1 \mathrm{~Hz}, 1 \mathrm{H}), 4.81(\mathrm{dd}, J=35.4,9.1 \mathrm{~Hz}, 1 \mathrm{H}), 3.65(\mathrm{~d}, J=17.1 \mathrm{~Hz}, 1 \mathrm{H}), 3.39(\mathrm{~d}$, $J=17.1 \mathrm{~Hz}, 1 \mathrm{H}), 2.13(\mathrm{~s}, 3 \mathrm{H}), 2.02(\mathrm{~d}, J=2.7 \mathrm{~Hz}, 6 \mathrm{H}), 1.61(\mathrm{~s}, 6 \mathrm{H}) ;{ }^{13} \mathrm{C} \mathrm{NMR}\left(126 \mathrm{MHz}, \mathrm{CDCl}_{3}\right) \delta$ 198.7 (s), 166.0 (d, $J=10.1 \mathrm{~Hz}), 152.5$ (s), 134.5 (s), 134.2 (s), 126.7 (s), 125.4 (s), 123.8 (s), 82.7 (d, $J=173.9 \mathrm{~Hz}), 81.9$ (s), $61.0(\mathrm{~d}, J=20.2 \mathrm{~Hz}), 40.0$ (s), 35.0 (s), 33.4 (s), 29.8 (s); ${ }^{19} \mathrm{~F}$ NMR (470 MHz, 
$\left.\mathrm{CDCl}_{3}\right) \delta-230.1(\mathrm{t}, J=47.0 \mathrm{~Hz})$; HR-MS (EI) Calcd. For 342.1631, $\mathrm{C}_{21} \mathrm{H}_{23} \mathrm{FO}_{3}$, found 342.1638.

methyl 6-(fluoromethyl)-5-oxo-6,7,8,9-tetrahydro-5H-benzo[7]annulene-6-carboxylate 3u<smiles></smiles>

Colorless oil, yield 61\% (30.5 mg). Eluent: ethyl acetate/ petroleum ether (1:40). ${ }^{1} \mathrm{H} \mathrm{NMR} \mathrm{(500} \mathrm{MHz,}$ $\left.\mathrm{CDCl}_{3}\right) \delta 7.44(\mathrm{~d}, J=7.6 \mathrm{~Hz}, 1 \mathrm{H}), 7.38(\mathrm{t}, J=7.5 \mathrm{~Hz}, 1 \mathrm{H}), 7.27(\mathrm{t}, J=7.5 \mathrm{~Hz}, 1 \mathrm{H}), 7.13(\mathrm{~d}, J=7.6 \mathrm{~Hz}$, $1 \mathrm{H}), 4.88(\mathrm{dd}, J=23.2,8.9 \mathrm{~Hz}, 1 \mathrm{H}), 4.79(\mathrm{dd}, J=23.0,8.9 \mathrm{~Hz}, 1 \mathrm{H}), 3.63(\mathrm{~s}, 3 \mathrm{H}), 3.04-2.95(\mathrm{~m}, 1 \mathrm{H})$, $2.84(\mathrm{ddd}, J=15.2,6.8,4.3 \mathrm{~Hz}, 1 \mathrm{H}), 2.53-2.44(\mathrm{~m}, 1 \mathrm{H}), 2.13-2.05(\mathrm{~m}, 1 \mathrm{H}), 2.04-1.91(\mathrm{~m}, 2 \mathrm{H})$; ${ }^{13} \mathrm{C} \mathrm{NMR}\left(126 \mathrm{MHz}, \mathrm{CDCl}_{3}\right) \delta 202.6$ (s), 170.1 (s), 139.4 (s), 138.8 (s), 131.8 (s), 129.3 (s), 128.9 (s), 126.7 (s), 86.4 (d, $J=177.7 \mathrm{~Hz}), 62.9$ (d, $J=19.0 \mathrm{~Hz}), 52.6$ (s), 32.9 (s), 29.8 (d, $J=3.2 \mathrm{~Hz}), 23.5$ (s); ${ }^{19} \mathrm{~F}$ NMR $\left(470 \mathrm{MHz}, \mathrm{CDCl}_{3}\right) \delta-229.3\left(\mathrm{t}, J=47.0 \mathrm{~Hz}\right.$ ); HR-MS (EI) Calcd. For 250.1005, $\mathrm{C}_{14} \mathrm{H}_{15} \mathrm{FO}_{3}$, found 250.0995 .

ethyl 3-(fluoromethyl)-4-oxochromane-3-carboxylate 3v<smiles>CCOC(=O)C1(C)COc2ccccc2C1=O</smiles>

Colorless oil, yield 72\% (36.3 mg). Eluent: ethyl acetate/ petroleum ether (1:40). ${ }^{1} \mathrm{H}$ NMR (500 MHz, $\left.\mathrm{CDCl}_{3}\right) \delta 7.95(\mathrm{dd}, J=7.9,1.4 \mathrm{~Hz}, 1 \mathrm{H}), 7.58-7.52(\mathrm{~m}, 1 \mathrm{H}), 7.09(\mathrm{t}, J=7.5 \mathrm{~Hz}, 1 \mathrm{H}), 7.04(\mathrm{~d}, J=8.4$ $\mathrm{Hz}, 1 \mathrm{H}), 5.00-4.93(\mathrm{~m}, 2 \mathrm{H}), 4.87(\mathrm{dd}, J=15.0,9.5 \mathrm{~Hz}, 1 \mathrm{H}), 4.66(\mathrm{~d}, J=11.8 \mathrm{~Hz}, 1 \mathrm{H}), 4.26(\mathrm{q}, J=$ $7.1 \mathrm{~Hz}, 2 \mathrm{H}), 1.25$ (t, $J=7.1 \mathrm{~Hz}, 3 \mathrm{H}) ;{ }^{13} \mathrm{C} \mathrm{NMR}\left(126 \mathrm{MHz}, \mathrm{CDCl}_{3}\right) \delta 187.5$ (s), 167.9 (d, $\left.J=4.7 \mathrm{~Hz}\right)$, 162.4 (s), 137.8 (s), 128.9 (s), 123.2 (s), 121.2 (s), 119.1 (s), 82.7 (d, $J=178.9$ Hz), 70.5 (d, $J=5.4$ $\mathrm{Hz}), 63.5(\mathrm{~s}), 59.5$ (d, $J=19.0 \mathrm{~Hz}), 15.0(\mathrm{~s}) ;{ }^{19} \mathrm{~F} \mathrm{NMR}\left(470 \mathrm{MHz}, \mathrm{CDCl}_{3}\right) \delta-230.2(\mathrm{t}, J=47.0 \mathrm{~Hz})$; HR-MS (EI) Calcd. For 252.0798, $\mathrm{C}_{13} \mathrm{H}_{13} \mathrm{FO}_{4}$, found 252.0791.

ethyl 3-(fluoromethyl)-4-oxothiochromane-3-carboxylate 3w<smiles>CCOC(=O)C1(C)CSc2ccccc2C1=O</smiles> 
Colorless oil, yield 69\% (36.9 mg). Eluent: ethyl acetate/ petroleum ether (1:40). ${ }^{1} \mathrm{H} \mathrm{NMR}(500 \mathrm{MHz}$, $\left.\mathrm{CDCl}_{3}\right) \delta 8.16(\mathrm{~d}, J=8.0 \mathrm{~Hz}, 1 \mathrm{H}), 7.44(\mathrm{t}, J=7.6 \mathrm{~Hz}, 1 \mathrm{H}), 7.29(\mathrm{~d}, J=7.6 \mathrm{~Hz}, 1 \mathrm{H}), 7.24(\mathrm{t}, J=7.6 \mathrm{~Hz}$, 1H), $5.08(\mathrm{dd}, J=46.1,9.1 \mathrm{~Hz}, 1 \mathrm{H}), 4.89(\mathrm{dd}, J=46.7,9.1 \mathrm{~Hz}, 1 \mathrm{H}), 4.32-4.22(\mathrm{~m}, 2 \mathrm{H}), 3.62(\mathrm{dd}, J$ $=48.3,13.7 \mathrm{~Hz}, 2 \mathrm{H}), 1.24(\mathrm{t}, J=7.1 \mathrm{~Hz}, 3 \mathrm{H}) ;{ }^{13} \mathrm{C} \mathrm{NMR}\left(126 \mathrm{MHz}, \mathrm{CDCl}_{3}\right) \delta 190.0(\mathrm{~s}), 168.9(\mathrm{~d}, J=$ $5.0 \mathrm{~Hz}), 142.3$ (s), 134.8 (s), 131.3 (s), 131.2 (s), 128.6 (s), 126.5 (s), 83.5 (d, J = 180.2 Hz), 63.5 (s), $59.6(\mathrm{~d}, J=18.7 \mathrm{~Hz}), 32.3(\mathrm{~d}, J=5.5 \mathrm{~Hz}), 15.1(\mathrm{~s}) ;{ }^{19} \mathrm{~F}$ NMR $\left(470 \mathrm{MHz}, \mathrm{CDCl}_{3}\right) \delta-228.5(\mathrm{t}, J=47.0$ $\mathrm{Hz}$ ); HR-MS (EI) Calcd. For 268.0569, $\mathrm{C}_{13} \mathrm{H}_{13} \mathrm{FO}_{3} \mathrm{~S}$, found 268.0567.

\section{Synthesis of ethyl 2-benzyl-2-(fluoromethyl)-3-oxo-3-phenylpropanoate 3y}<smiles>CCOC(=O)C(C)(Br)C(=O)c1ccccc1</smiles>

A mixture of $2 \mathbf{y}(56.4 \mathrm{mg}, 0.2 \mathrm{mmol})$, fluoromethyl iodide $\left(\mathrm{ICH}_{2} \mathrm{~F}, 80 \mathrm{mg}, 0.5 \mathrm{mmol}\right), t \mathrm{BuOLi}(32 \mathrm{mg}$, $0.4 \mathrm{mmol}$ ) in $2.0 \mathrm{~mL}$ DMF was stirred at room temperature for 2 hours. After completion, the reaction mixture was diluted with ethyl acetate, and then washed with water and brine. After drying over $\mathrm{Na}_{2} \mathrm{SO}_{4}$, the solvent was removed under reduced pressure, and the residue was subject to chromatography on silica gel to afford monofluoromethylated products $\mathbf{3 y}$.

Colorless oil, yield 31\% (19.5 mg). Eluent: ethyl acetate/ petroleum ether (1:20). ${ }^{1} \mathrm{H} \mathrm{NMR}$ (500 MHz, $\left.\mathrm{CDCl}_{3}\right) \delta 7.89(\mathrm{~d}, J=7.9 \mathrm{~Hz}, 2 \mathrm{H}), 7.59(\mathrm{t}, J=7.4 \mathrm{~Hz}, 1 \mathrm{H}), 7.48(\mathrm{t}, J=7.7 \mathrm{~Hz}, 2 \mathrm{H}), 7.24-7.18(\mathrm{~m}$, $3 \mathrm{H}), 7.01-6.94(\mathrm{~m}, 2 \mathrm{H}), 4.90-4.85(\mathrm{~m}, 1 \mathrm{H}), 4.80-4.76(\mathrm{~m}, 1 \mathrm{H}), 4.25-4.16(\mathrm{~m}, 2 \mathrm{H}), 3.59(\mathrm{~s}, 2 \mathrm{H})$, $1.12(\mathrm{t}, J=7.1 \mathrm{~Hz}, 3 \mathrm{H}) .{ }^{13} \mathrm{C} \mathrm{NMR}\left(126 \mathrm{MHz}, \mathrm{CDCl}_{3}\right) \delta 195.7$ (s), 171.5 (s), 136.8 (s), 136.0 (s), 134.3 (s), 131.3 (s), 129.9 (s), 129.5 (s), 129.4 (s), 128.3 (s), 84.4 (d, $J=172.6 \mathrm{~Hz}$ ), 63.8 (d, $J=20.2 \mathrm{~Hz}$ ), 63.1 (s), 38.0 (s), 14.8 (s). ${ }^{19} \mathrm{~F}$ NMR (470 MHz, $\left.\mathrm{CDCl}_{3}\right) \delta-233.1$ (t, $\left.J=47.0 \mathrm{~Hz}\right)$. HR-MS (EI) Calcd. For 314.1318, $\mathrm{C}_{19} \mathrm{H}_{19} \mathrm{FO}_{3}$, found 314.1314.

\section{General procedure for carbon-selective monofluoromethylation of malonates}

A mixture of malonates $\mathbf{2 z}$ or $2 \mathbf{a a}(0.2 \mathrm{mmol})$, fluoromethyl iodide $\left(\mathrm{ICH}_{2} \mathrm{~F}, 80 \mathrm{mg}, 0.5 \mathrm{mmol}\right), \mathrm{Cs}_{2} \mathrm{CO}_{3}$ (130 mg, $0.4 \mathrm{mmol})$ in $2.0 \mathrm{~mL}$ DMF was stirred at room temperature for 2 hours. After completion, the reaction mixture was diluted with ethyl acetate, and then washed with water and brine. After drying 
over $\mathrm{Na}_{2} \mathrm{SO}_{4}$, the solvent was removed under reduced pressure, and the residue was subject to chromatography on silica gel to afford monofluoromethylated products $\mathbf{3 z}$ or $\mathbf{3 a a}$.

diethyl 2-(fluoromethyl)-2-phenylmalonate $3 z^{17}$

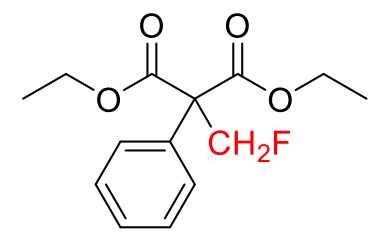

Yellow oil, yield 91\% (48.8 mg). Eluent: ethyl acetate/ petroleum ether (1:20). ${ }^{1} \mathrm{H} \mathrm{NMR}(500 \mathrm{MHz}$, $\left.\mathrm{CDCl}_{3}\right) \delta 7.43-7.32(\mathrm{~m}, 5 \mathrm{H}), 5.12(\mathrm{~d}, J=46.5 \mathrm{~Hz}, 2 \mathrm{H}), 4.35-4.24(\mathrm{~m}, 4 \mathrm{H}), 1.29(\mathrm{t}, J=7.1 \mathrm{~Hz}, 6 \mathrm{H})$. ${ }^{19} \mathrm{~F}$ NMR $\left(470 \mathrm{MHz}, \mathrm{CDCl}_{3}\right) \delta-222.9(\mathrm{t}, J=47.0 \mathrm{~Hz})$.

diethyl 2-benzyl-2-(fluoromethyl)malonate 3aa ${ }^{17}$<smiles>CCOC(=O)C(C)(Cc1ccccc1)C(=O)OCC</smiles>

Yellow oil, yield 90\% (50.8 mg). Eluent: ethyl acetate/ petroleum ether (1:20). ${ }^{1} \mathrm{H} \mathrm{NMR}(500 \mathrm{MHz}$, $\left.\mathrm{CDCl}_{3}\right) \delta 7.27(\mathrm{dt}, J=8.2,6.9 \mathrm{~Hz}, 3 \mathrm{H}), 7.15(\mathrm{~d}, J=6.8 \mathrm{~Hz}, 2 \mathrm{H}), 4.68(\mathrm{~d}, J=46.8 \mathrm{~Hz}, 2 \mathrm{H}), 4.23(\mathrm{qd}, J$ $=7.1,1.8 \mathrm{~Hz}, 4 \mathrm{H}), 3.38(\mathrm{~s}, 2 \mathrm{H}), 1.26(\mathrm{t}, J=7.1 \mathrm{~Hz}, 6 \mathrm{H}) .{ }^{19} \mathrm{~F} \mathrm{NMR}\left(470 \mathrm{MHz}, \mathrm{CDCl}_{3}\right) \delta-231.1(\mathrm{t}, J=$ $47.0 \mathrm{~Hz})$.

\section{Gram-scale Reactions}<smiles>COC(=O)C1CCc2ccccc2C1=O</smiles>

2a

5 mmol, 1 equiv<smiles>COC(=O)C1Cc2ccccc2C1=O</smiles>

$2 \mathrm{~h}$
tBuOLi (2 equiv)

diglyme, rt, $2 \mathrm{~h}$

(2.5 equiv)

$5 \mathrm{mmol}, 1$ equiv<smiles>COC(=O)C1(C)CCc2ccccc2C1=O</smiles>

$3 a$ $69 \%$ isolated yield<smiles>COC(=O)C1(C)Cc2ccccc2C1=O</smiles>

3h

$78 \%$ isolated yield

To a $100 \mathrm{~mL}$ of flask was added $\mathbf{2 a}$ or $\mathbf{2 h}\left(5 \mathrm{mmol}, 1\right.$ equiv), fluoromethyl iodide $\left(\mathrm{ICH}_{2} \mathrm{~F}, 2.0 \mathrm{~g}, 12.5\right.$ 
mmol, 2.5 equiv), $t \mathrm{BuOLi}(0.8 \mathrm{~g}, 10 \mathrm{mmol}, 2$ equiv) and diglyme $(25 \mathrm{~mL})$. The reaction mixture was stirred at room temperature for 2 hours. After completion, the reaction mixture was diluted with ethyl acetate, and then washed with water and brine. After drying over $\mathrm{Na}_{2} \mathrm{SO}_{4}$, the solvent was removed under reduced pressure, and the residue was subject to chromatography on silica gel to afford monofluoromethylated products $\mathbf{3 a}(69 \%, 0.82 \mathrm{~g})$ or $\mathbf{3 h}(78 \%, 0.87 \mathrm{~g})$.

\section{Synthesis of methyl 2-(fluoromethyl)-2,3-dihydro-1H-indene-2-carboxylate 5}

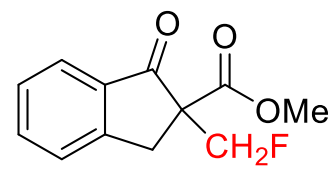

$3 \mathrm{~h}$

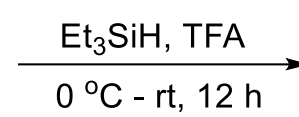

$0^{\circ} \mathrm{C}-\mathrm{rt}, 12 \mathrm{~h}$

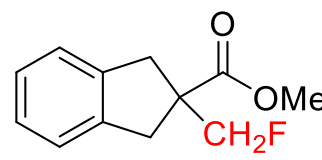

5

To a solution of methyl 2-(fluoromethyl)-1-oxo-2,3-dihydro-1H-indene-2-carboxylate $\mathbf{3 h}$ (36 mg, 0.16 $\mathrm{mmol})$ in trifluoroacetic acid $(1 \mathrm{~mL})$, was added dropwise triethylsilane $(0.13 \mathrm{~mL}, 0.84 \mathrm{mmol})$ at $0{ }^{\circ} \mathrm{C}$, and the reaction mixture was stirred for 2 hours at the same temperature. Then the reaction was allowed to warm to room temperature and stirred overnight. After that, reaction was cooled to $0{ }^{\circ} \mathrm{C}$, quenched with aqueous $\mathrm{NaHCO}_{3}$ carefully, and extracted with ethyl acetate three times. The combined organic layer washed with brine, dried over anhydrous $\mathrm{Na}_{2} \mathrm{SO}_{4}$, filtered, and concentrated under reduced pressure. The residue was purified by silica gel column chromatography (ethyl acetate/ petroleum ether $=1: 100)$ to give product $5(24.6 \mathrm{mg}, 74 \%)$ as a colorless oil. ${ }^{1} \mathrm{H} \mathrm{NMR}\left(500 \mathrm{MHz}, \mathrm{CDCl}_{3}\right) \delta 7.20(\mathrm{dq}, J$ $=9.1,4.4 \mathrm{~Hz}, 4 \mathrm{H}), 4.53(\mathrm{~d}, J=47.0 \mathrm{~Hz}, 2 \mathrm{H}), 3.78(\mathrm{~s}, 3 \mathrm{H}), 3.47(\mathrm{dd}, J=16.3,1.8 \mathrm{~Hz}, 2 \mathrm{H}), 3.05(\mathrm{~d}, J=$ $16.4 \mathrm{~Hz}, 2 \mathrm{H}) .{ }^{13} \mathrm{C}$ NMR $\left(126 \mathrm{MHz}, \mathrm{CDCl}_{3}\right) \delta 176.0$ (s), 141.2 (s), 128.1 (s), 125.8 (s), 86.9 (d, J=176.4 $\mathrm{Hz}), 55.3(\mathrm{~d}, J=18.2 \mathrm{~Hz}), 53.6(\mathrm{~s}), 39.5(\mathrm{~d}, J=5.4 \mathrm{~Hz}) .{ }^{19} \mathrm{~F} \mathrm{NMR}\left(470 \mathrm{MHz}, \mathrm{CDCl}_{3}\right) \delta-218.4(\mathrm{t}, J=$ 47.0 Hz). HR-MS (EI) Calcd. For 208.0900, $\mathrm{C}_{12} \mathrm{H}_{13} \mathrm{FO}_{2}$, found 208.0906.

\section{Synthesis of 2-(fluoromethyl)-2-(hydroxymethyl)-6-methyl-2,3-dihydro-1H-inden-1-ol 6}<smiles>COC(=O)C1(C)Cc2ccc(C)cc2C1=O</smiles>

$3 \mathbf{i}$

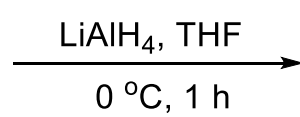

$0^{\circ} \mathrm{C}, 1 \mathrm{~h}$

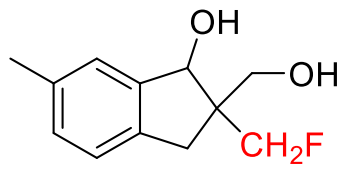

6

$3 \mathbf{i}(40 \mathrm{mg}, 0.17 \mathrm{mmol})$ in anhydrous THF $(1 \mathrm{~mL})$ was added slowly to the mixture of lithium aluminum hydride $(19 \mathrm{mg}, 0.5 \mathrm{mmol})$ in anhydrous THF $(1 \mathrm{~mL})$ at $0{ }^{\circ} \mathrm{C}$. After stirring for another 1 hour at the same temperature, reaction was quenched by the dropwise addition of EtOAc followed by a $10 \% \mathrm{HCl}$. 
After vigorous stirring for another $20 \mathrm{~min}$, the resulting mixture was extracted with ethyl acetate. The combined organic layers were washed with brine, dried over anhydrous $\mathrm{Na}_{2} \mathrm{SO}_{4}$ and concentrated in vacuum. The residue was purified by silica gel column chromatography (ethyl acetate/ petroleum ether $=1: 3)$ to give product $6(28.2 \mathrm{mg}, 79 \%, \mathrm{dr}=12: 1)$ as a yellow oil. ${ }^{1} \mathrm{H} \mathrm{NMR}\left(500 \mathrm{MHz}, \mathrm{CDCl}_{3}\right) \delta 7.20$ (s, 1H), $7.13-7.06(\mathrm{~m}, 2 \mathrm{H}), 5.14(\mathrm{~s}, 1 \mathrm{H}), 4.85(\mathrm{dd}, J=47.9,9.3 \mathrm{~Hz}, 1 \mathrm{H}), 4.52(\mathrm{dd}, J=47.3,9.3 \mathrm{~Hz}$, 1H), $3.80(\mathrm{~d}, J=10.7 \mathrm{~Hz}, 1 \mathrm{H}), 3.69(\mathrm{~d}, J=10.7 \mathrm{~Hz}, 1 \mathrm{H}), 2.89(\mathrm{~d}, J=16.2 \mathrm{~Hz}, 1 \mathrm{H}), 2.63(\mathrm{~d}, J=16.2$ $\mathrm{Hz}, 1 \mathrm{H}), 2.35$ (s, 3H), 2.20 - $1.71(\mathrm{br}, 2 \mathrm{H}) .{ }^{13} \mathrm{C} \mathrm{NMR}\left(126 \mathrm{MHz}, \mathrm{CDCl}_{3}\right) \delta 144.2$ (s), $138.1(\mathrm{~s}), 138.0$ (s), 130.7 (s), 126.2 (s), 126.1 (s), 85.1 (d, $J=166.3 \mathrm{~Hz}), 80.6$ (d, $J=4.9 \mathrm{~Hz}), 66.5$ (d, $J=4.5 \mathrm{~Hz}), 54.3$ $(\mathrm{d}, J=16.1 \mathrm{~Hz}), 35.9(\mathrm{~d}, J=6.0 \mathrm{~Hz}), 22.3(\mathrm{~s}) .{ }^{19} \mathrm{~F} \mathrm{NMR}\left(470 \mathrm{MHz}, \mathrm{CDCl}_{3}\right) \delta-231.5(\mathrm{t}, J=47.0 \mathrm{~Hz})$. HR-MS (EI) Calcd. For 210.1056, $\mathrm{C}_{12} \mathrm{H}_{15} \mathrm{FO}_{2}$, found 210.1060 . 


\section{Table S2: Survey for DAAA reaction of product 3r}<smiles>C=CCOC(=O)C1(C)Cc2ccccc2C1=O</smiles>

$3 r$<smiles>c1ccc(-c2c(P(c3ccccc3)c3ccccc3)ccc3ccccc23)cc1</smiles>

L1

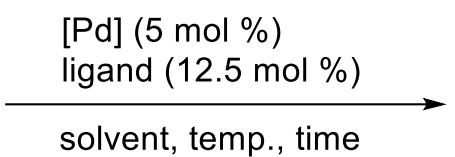<smiles>Pc1ccccc1-c1c(-c2ccccc2)ccc2c1OCO2</smiles>

L2<smiles>C=CCC1(C)Cc2ccccc2C1=O</smiles>

7<smiles>CC(C)(C)[C@H]1COC(c2ccccc2P)=N1</smiles>

L3<smiles>O=C(NC1CCCCC1)c1ccccc1Pc1ccccc1</smiles><smiles>O=C(NC(Cc1ccccc1)c1ccccc1)c1ccccc1Pc1ccccc1</smiles>

L4

L5

\begin{tabular}{llllllll}
\hline Entry & {$[\mathrm{Pd}]$} & ligand & solvent & $\begin{array}{l}\text { temp. } \\
\left({ }^{\circ} \mathrm{C}\right)\end{array}$ & $\begin{array}{c}\text { Time } \\
(\mathrm{h})\end{array}$ & $\begin{array}{c}\text { Yield } \\
(\%)\end{array}$ & $\begin{array}{c}\text { ee } \\
(\%)\end{array}$ \\
\hline 1 & $\mathrm{Pd}\left(\mathrm{PPh}_{3}\right)_{4}$ & $\mathrm{~L} 1$ & $\mathrm{THF}$ & $\mathrm{rt}$ & 2 & $\mathrm{NR}$ & - \\
2 & $\mathrm{Pd}(\mathrm{dba})_{2}$ & $\mathrm{~L} 1$ & $\mathrm{THF}$ & $\mathrm{rt}$ & 2 & $\mathrm{NR}$ & - \\
3 & $\mathrm{Pd}_{2}(\mathrm{dba})_{3}$ & $\mathrm{~L} 1$ & $\mathrm{THF}$ & $\mathrm{rt}$ & 2 & $\mathrm{NR}$ & - \\
4 & $\mathrm{Pd}_{2}(\mathrm{dba})_{3}$ & $\mathrm{~L} 2$ & $\mathrm{THF}$ & $\mathrm{rt}$ & 2 & $\mathrm{NR}$ & - \\
5 & $\mathrm{Pd}_{2}(\mathrm{dba})_{3}$ & $\mathrm{~L} 3$ & $\mathrm{THF}$ & $\mathrm{rt}$ & 2 & $\mathrm{NR}$ & - \\
6 & $\mathrm{Pd}_{2}(\mathrm{dba})_{3}$ & $\mathrm{~L} 4$ & $\mathrm{THF}$ & $\mathrm{rt}$ & 2 & $\mathrm{NR}$ & - \\
7 & $\mathrm{Pd}_{2}(\mathrm{dba})_{3}$ & $\mathrm{~L} 5$ & $\mathrm{THF}$ & $\mathrm{rt}$ & 2 & $\mathrm{NR}$ & - \\
8 & $\mathrm{Pd}_{2}(\mathrm{dba})_{3}$ & $\mathrm{~L} 5$ & DCM & $\mathrm{rt}$ & 2 & $\mathrm{NR}$ & - \\
9 & $\mathrm{Pd}_{2}(\mathrm{dba})_{3}$ & $\mathrm{~L} 5$ & dioxane & $\mathrm{rt}$ & 2 & $\mathrm{NR}$ & - \\
10 & $\mathrm{Pd}_{2}(\mathrm{dba})_{3}$ & $\mathrm{~L} 5$ & TBME & $\mathrm{rt}$ & 2 & $\mathrm{NR}$ & - \\
11 & $\mathrm{Pd}_{2}(\mathrm{dba})_{3}$ & $\mathrm{~L} 5$ & dioxane & 0 & 2 & $\mathrm{NR}$ & - \\
12 & $\mathrm{Pd}_{2}(\mathrm{dba})_{3}$ & $\mathrm{~L} 5$ & dioxane & -20 & 2 & $\mathrm{NR}$ & - \\
13 & $\mathrm{Pd}_{2}(\mathrm{dba})_{3}$ & $\mathrm{~L} 5$ & dioxane & 50 & 2 & $\mathrm{NR}$ & - \\
14 & $\mathrm{Pd}_{2}(\mathrm{dba})_{3}$ & $\mathrm{~L} 5$ & dioxane & 0 & 8 & $\mathrm{NR}$ & - \\
15 & $\mathrm{Pd}_{2}(\mathrm{dba})_{3}$ & $\mathrm{~L} 5$ & dioxane & 0 & 24 & $\mathrm{NR}$ & - \\
\hline $\operatorname{Rin}^{2}$ & & & & 2 & - \\
\hline
\end{tabular}

Reaction conditions: $3 \mathrm{r}(0.1 \mathrm{mmol}, 1.0$ equiv), [Pd] (5 mol \%), ligand (12.5 mol \%) in solvent $(1 \mathrm{~mL})$ at prescribed temperature. $\mathrm{dba}=$ dibenzylideneacetone, $\mathrm{DCM}=$ dichloromethane, $\mathrm{TBME}=$ tert -butyl methyl ether, $\mathrm{NR}=$ no reaction.

According to the reaction results, we concluded that DAAA reaction was not suitable for the synthesis of chiral monofluoromethylated compounds and further investigation is required. 


\section{DFT calculation}

Computational details: All density functional theory (DFT) calculations were performed using Gaussian 09. ${ }^{18}$ The geometries and frequency calculations were performed using the M06-2 $\mathrm{X}^{19}$ density functional in conjunction with the $6-311 \mathrm{G}^{*}$ basis set. Thermal free energy corrections were obtained at $298.15 \mathrm{~K}$ to match the experimental conditions.

\section{M06-2X/6-311G* calculated Cartesian coordinates and energies}

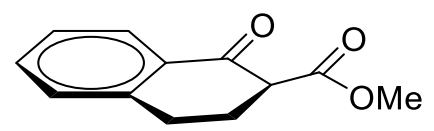

Standard orientation:

\begin{tabular}{|c|c|c|c|c|c|}
\hline \multirow{2}{*}{$\begin{array}{l}\text { Center } \\
\text { Number }\end{array}$} & \multirow{2}{*}{$\begin{array}{l}\text { Atomic } \\
\text { Number }\end{array}$} & \multirow{2}{*}{$\begin{array}{l}\text { Atomic } \\
\text { Type }\end{array}$} & \multicolumn{3}{|c|}{ Coordinates (Angstroms) } \\
\hline & & & $\mathrm{X}$ & $\mathrm{Y}$ & Z \\
\hline 1 & 6 & 0 & 4.018414 & -0.075306 & 0.319434 \\
\hline 2 & 6 & 0 & 3.171073 & 1.019401 & 0.222879 \\
\hline 3 & 6 & 0 & 1.805167 & 0.850062 & -0.005676 \\
\hline 4 & 6 & 0 & 1.306720 & -0.453014 & -0.132357 \\
\hline 5 & 6 & 0 & 2.161124 & -1.553921 & -0.028100 \\
\hline 6 & 6 & 0 & 3.514467 & -1.369688 & 0.193936 \\
\hline 7 & 1 & 0 & 1.254393 & 2.844092 & 0.537540 \\
\hline 8 & 1 & 0 & 5.076678 & 0.079324 & 0.499274 \\
\hline 9 & 1 & 0 & 3.570473 & 2.023048 & 0.332105 \\
\hline 10 & 6 & 0 & 0.889208 & 2.046310 & -0.113753 \\
\hline 11 & 1 & 0 & 1.733159 & -2.544767 & -0.128740 \\
\hline 12 & 1 & 0 & 4.177643 & -2.223329 & 0.270732 \\
\hline 13 & 6 & 0 & -1.017959 & 0.516357 & -0.630234 \\
\hline 14 & 6 & 0 & -0.554388 & 1.693864 & 0.233232 \\
\hline 15 & 1 & 0 & -0.633946 & 1.416934 & 1.287752 \\
\hline 16 & 6 & 0 & -0.142005 & -0.710998 & -0.384811 \\
\hline 17 & 8 & 0 & -0.600757 & -1.825737 & -0.415800 \\
\hline 18 & 8 & 0 & -2.719005 & -0.054259 & 0.886104 \\
\hline 19 & 6 & 0 & -2.465320 & 0.134667 & -0.416030 \\
\hline 20 & 8 & 0 & -3.284388 & 0.014967 & -1.281253 \\
\hline 21 & 6 & 0 & -4.035028 & -0.519850 & 1.183217 \\
\hline 22 & 1 & 0 & -4.080171 & -0.609438 & 2.264953 \\
\hline 23 & 1 & 0 & -4.779796 & 0.188876 & 0.821423 \\
\hline 24 & 1 & 0 & -4.201485 & -1.487351 & 0.709586 \\
\hline 25 & 1 & 0 & -0.916375 & 0.773573 & -1.691079 \\
\hline 26 & 1 & 0 & -1.207721 & 2.554480 & 0.071611 \\
\hline 27 & 1 & 0 & 0.931505 & 2.437485 & -1.137975 \\
\hline
\end{tabular}


Thermal correction to Energy=

$$
\begin{gathered}
0.233414 \\
0.234358
\end{gathered}
$$

Thermal correction to Enthalpy=

Thermal correction to Gibbs Free Energy=

0.180896

Sum of electronic and zero-point Energies $=$

$-689.859744$

$-689.847005$

$-689.846061$

Sum of electronic and thermal Enthalpies=

$-689.899523$

\begin{tabular}{|c|c|c|c|c|c|}
\hline \multirow{2}{*}{$\begin{array}{l}\text { Center } \\
\text { Number }\end{array}$} & \multirow{2}{*}{$\begin{array}{l}\text { Atomic } \\
\text { Number }\end{array}$} & \multirow{2}{*}{$\begin{array}{l}\text { Atomic } \\
\text { Type }\end{array}$} & \multicolumn{3}{|c|}{ Coordinates (Angstroms) } \\
\hline & & & $\mathrm{X}$ & $\mathrm{Y}$ & Z \\
\hline 1 & 6 & 0 & -3.765151 & 1.013478 & -0.311919 \\
\hline 2 & 6 & 0 & -2.427719 & 1.371758 & -0.218874 \\
\hline 3 & 6 & 0 & -1.451337 & 0.403853 & 0.017023 \\
\hline 4 & 6 & 0 & -1.819354 & -0.937302 & 0.180927 \\
\hline 5 & 6 & 0 & -3.164265 & -1.285129 & 0.091505 \\
\hline 6 & 6 & 0 & -4.133449 & -0.319839 & -0.158012 \\
\hline 7 & 1 & 0 & -4.519444 & 1.769086 & -0.501386 \\
\hline 8 & 1 & 0 & -2.112844 & 2.402613 & -0.325934 \\
\hline 9 & 6 & 0 & -0.746527 & -1.946009 & 0.494335 \\
\hline 10 & 1 & 0 & -3.454109 & -2.323699 & 0.219583 \\
\hline 11 & 1 & 0 & -5.176584 & -0.608213 & -0.230577 \\
\hline 12 & 6 & 0 & 0.545511 & -1.614198 & -0.245113 \\
\hline 13 & 6 & 0 & 0.960164 & -0.176476 & -0.005106 \\
\hline 14 & 1 & 0 & -0.554146 & -1.930597 & 1.574558 \\
\hline 15 & 1 & 0 & 1.337580 & -2.286803 & 0.082633 \\
\hline 16 & 6 & 0 & -0.018086 & 0.823903 & 0.104673 \\
\hline 17 & 8 & 0 & 0.195544 & 2.070019 & 0.244659 \\
\hline 18 & 8 & 0 & 2.857647 & 1.288578 & 0.169745 \\
\hline 19 & 6 & 0 & 2.341126 & 0.163113 & -0.002902 \\
\hline 20 & 8 & 0 & 3.164451 & -0.881480 & -0.219531 \\
\hline 21 & 6 & 0 & 4.556968 & -0.597168 & -0.230233 \\
\hline 22 & 1 & 0 & 5.046580 & -1.549546 & -0.417966 \\
\hline 23 & 1 & 0 & 4.800243 & 0.116444 & -1.017936 \\
\hline 24 & 1 & 0 & 4.877895 & -0.188255 & 0.728321 \\
\hline 25 & 3 & 0 & 1.811036 & 2.711319 & 0.369115 \\
\hline 26 & 1 & 0 & -1.089862 & -2.952785 & 0.242729 \\
\hline 27 & 1 & 0 & 0.403162 & -1.799042 & -1.318189 \\
\hline
\end{tabular}

Standard orientation: 
Thermal correction to Energy=

0.224398

Thermal correction to Enthalpy=

0.225342

Thermal correction to Gibbs Free Energy=

Sum of electronic and zero-point Energies $=$

0.171142

$-696.884371$

$-696.871018$

$-696.870074$

Sum of electronic and thermal Enthalpies=

$-696.924274$

Sum of electronic and thermal Free Energies=

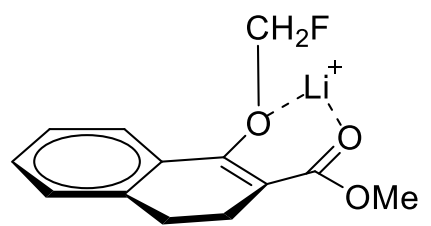

Standard orientation:

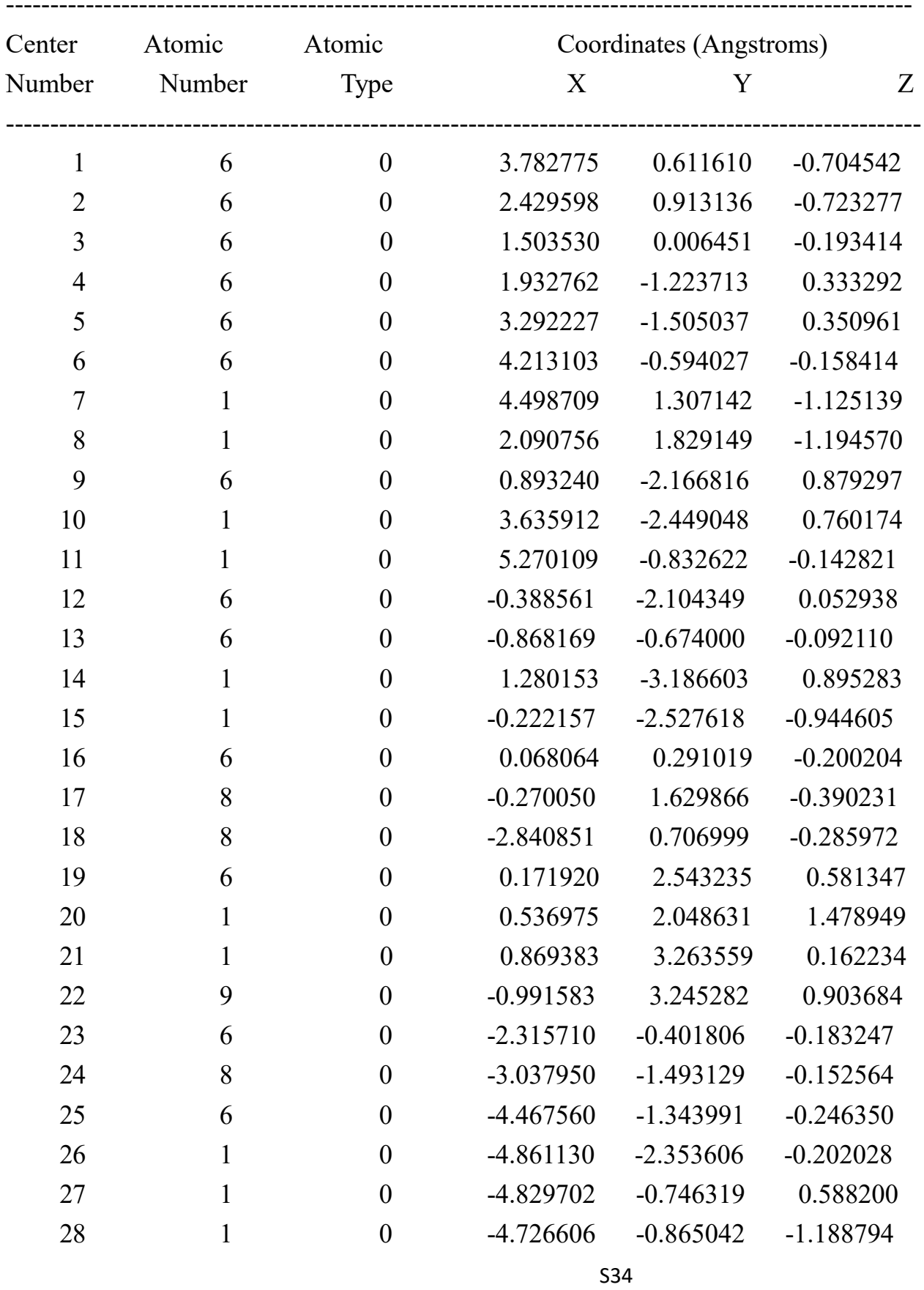




$\begin{array}{rrrrrr}29 & 3 & 0 & -2.138149 & 2.347278 & -0.403028 \\ 30 & 1 & 0 & 0.670325 & -1.894405 & 1.918303 \\ 31 & 1 & 0 & -1.166549 & -2.702755 & 0.523218\end{array}$

Zero-point correction $=$

Thermal correction to Energy=

Thermal correction to Enthalpy=

Thermal correction to Gibbs Free Energy=

Sum of electronic and zero-point Energies=

Sum of electronic and thermal Energies=

Sum of electronic and thermal Enthalpies=

Sum of electronic and thermal Free Energies=

0.245472 (Hartree/Particle)
0.261306
0.262250
0.202252
-835.753080
-835.737246
-835.736302
-835.796301

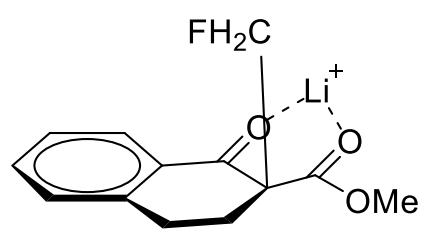

Standard orientation:

\begin{tabular}{|c|c|c|c|c|c|}
\hline \multirow{2}{*}{$\begin{array}{l}\text { Center } \\
\text { Number }\end{array}$} & \multirow{2}{*}{$\begin{array}{l}\text { Atomic } \\
\text { Number }\end{array}$} & \multirow{2}{*}{$\begin{array}{l}\text { Atomic } \\
\text { Type }\end{array}$} & \multicolumn{3}{|c|}{ Coordinates (Angstroms) } \\
\hline & & & $X$ & $\mathrm{Y}$ & Z \\
\hline 1 & 6 & 0 & 4.328380 & -0.234077 & -0.359139 \\
\hline 2 & 6 & 0 & 3.348664 & -1.216892 & -0.354035 \\
\hline 3 & 6 & 0 & 2.004193 & -0.884002 & -0.211030 \\
\hline 4 & 6 & 0 & 1.665213 & 0.473959 & -0.063418 \\
\hline 5 & 6 & 0 & 2.663390 & 1.465385 & -0.068905 \\
\hline 6 & 6 & 0 & 3.988236 & 1.113864 & -0.219172 \\
\hline 7 & 1 & 0 & 0.904859 & -2.417567 & 0.792927 \\
\hline 8 & 1 & 0 & 5.368140 & -0.516537 & -0.478874 \\
\hline 9 & 1 & 0 & 3.627318 & -2.258607 & -0.471100 \\
\hline 10 & 6 & 0 & 0.949500 & -1.957528 & -0.201014 \\
\hline 11 & 1 & 0 & 2.372921 & 2.502120 & 0.047399 \\
\hline 12 & 1 & 0 & 4.758771 & 1.874713 & -0.228496 \\
\hline 13 & 6 & 0 & -0.812026 & -0.178207 & 0.195286 \\
\hline 14 & 6 & 0 & -0.417997 & -1.426556 & -0.608745 \\
\hline 15 & 1 & 0 & -0.408837 & -1.169581 & -1.671166 \\
\hline 16 & 6 & 0 & 0.281832 & 0.899090 & 0.138256 \\
\hline 17 & 8 & 0 & 0.002641 & 2.081633 & 0.337793 \\
\hline 18 & 8 & 0 & -2.828354 & -0.435008 & -1.020112 \\
\hline 19 & 6 & 0 & -4.165458 & -0.047174 & -1.408208 \\
\hline 20 & 1 & 0 & -4.127349 & 0.882167 & -1.972953 \\
\hline 21 & 1 & 0 & -4.527730 & -0.863668 & -2.022699 \\
\hline 22 & 1 & 0 & -4.777429 & 0.077016 & -0.517036 \\
\hline 23 & 6 & 0 & -0.975554 & -0.515191 & 1.696852 \\
\hline & & & & & \\
\hline
\end{tabular}




$\begin{array}{lllrrc}24 & 1 & 0 & -1.280965 & 0.367451 & 2.263342 \\ 25 & 9 & 0 & -1.955021 & -1.467549 & 1.816336 \\ 26 & 6 & 0 & -2.170244 & 0.372266 & -0.244904 \\ 27 & 8 & 0 & -2.627603 & 1.444843 & 0.130714 \\ 28 & 1 & 0 & -0.045301 & -0.909404 & 2.109452 \\ 29 & 3 & 0 & -1.610687 & 2.889513 & 0.605460 \\ 30 & 1 & 0 & 1.242304 & -2.759522 & -0.881279 \\ 31 & 1 & 0 & -1.181700 & -2.190541 & -0.471026\end{array}$

Zero-point correction $=$

Thermal correction to Energy=

Thermal correction to Enthalpy=

Thermal correction to Gibbs Free Energy=

Sum of electronic and zero-point Energies=

Sum of electronic and thermal Energies=

Sum of electronic and thermal Enthalpies=

Sum of electronic and thermal Free Energies=

0.245032 (Hartree/Particle)
0.260852
0.261796
0.201650
-835.771372
-835.755552
-835.754607
-835.814754

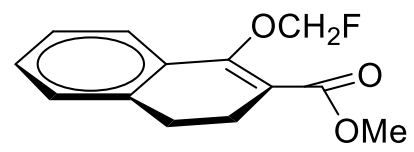

Standard orientation:

\begin{tabular}{|c|c|c|c|c|c|}
\hline \multirow{2}{*}{$\begin{array}{l}\text { Center } \\
\text { Number }\end{array}$} & \multirow{2}{*}{$\begin{array}{l}\text { Atomic } \\
\text { Number }\end{array}$} & \multirow{2}{*}{$\begin{array}{l}\text { Atomic } \\
\text { Type }\end{array}$} & \multicolumn{3}{|c|}{ Coordinates (Angstroms) } \\
\hline & & & $\mathrm{X}$ & $\mathrm{Y}$ & Z \\
\hline 1 & 6 & 0 & 3.542278 & 0.438599 & 1.030476 \\
\hline 2 & 6 & 0 & 3.990653 & -0.589531 & 0.209057 \\
\hline 3 & 6 & 0 & 3.088955 & -1.286172 & -0.590883 \\
\hline 4 & 6 & 0 & 1.738668 & -0.960598 & -0.587382 \\
\hline 5 & 6 & 0 & 1.292053 & 0.089408 & 0.230890 \\
\hline 6 & 6 & 0 & 2.194380 & 0.775885 & 1.043187 \\
\hline 7 & 6 & 0 & 0.718898 & -1.678077 & -1.436583 \\
\hline 8 & 6 & 0 & -0.588737 & -1.843765 & -0.662321 \\
\hline 9 & 6 & 0 & -1.056690 & -0.491700 & -0.165295 \\
\hline 10 & 6 & 0 & -0.148100 & 0.405593 & 0.229770 \\
\hline 11 & 6 & 0 & -2.519354 & -0.158121 & -0.170118 \\
\hline 12 & 8 & 0 & -2.993870 & 0.730429 & -0.806949 \\
\hline 13 & 8 & 0 & -3.330896 & -0.967789 & 0.550309 \\
\hline 14 & 6 & 0 & -2.798967 & -1.861571 & 1.518213 \\
\hline 15 & 8 & 0 & -0.540996 & 1.613333 & 0.759920 \\
\hline 16 & 1 & 0 & 4.239239 & 0.973655 & 1.665026 \\
\hline 17 & 1 & 0 & 5.041560 & -0.855943 & 0.196544 \\
\hline 18 & 1 & 0 & 3.439186 & -2.095436 & -1.223954 \\
\hline 19 & 1 & 0 & 1.831211 & 1.559727 & 1.698897 \\
\hline \multirow[t]{2}{*}{20} & 1 & 0 & 1.104601 & -2.648485 & -1.756002 \\
\hline & & \multicolumn{4}{|c|}{ S36 } \\
\hline
\end{tabular}




$\begin{array}{lllrrc}21 & 1 & 0 & 0.522859 & -1.087220 & -2.339232 \\ 22 & 1 & 0 & -0.427353 & -2.527159 & 0.180783 \\ 23 & 1 & 0 & -1.355015 & -2.294002 & -1.298223 \\ 24 & 1 & 0 & -3.571763 & -1.982187 & 2.274166 \\ 25 & 1 & 0 & -1.896853 & -1.461642 & 1.985133 \\ 26 & 1 & 0 & -2.584405 & -2.833323 & 1.068600 \\ 27 & 6 & 0 & -0.184987 & 2.729663 & 0.004421 \\ 28 & 1 & 0 & -0.513811 & 3.595139 & 0.576817 \\ 29 & 1 & 0 & 0.889393 & 2.747243 & -0.198865 \\ 30 & 9 & 0 & -0.823005 & 2.720464 & -1.197391\end{array}$

Zero-point correction $=$ 0.241474 (Hartree/Particle)

Thermal correction to Energy=

0.256666

Thermal correction to Enthalpy=

0.257611

Thermal correction to Gibbs Free Energy=

0.197431

Sum of electronic and zero-point Energies=

$-828.336499$

Sum of electronic and thermal Energies=

$-828.321307$

Sum of electronic and thermal Enthalpies=

$-828.320363$

Sum of electronic and thermal Free Energies=

$-828.380542$

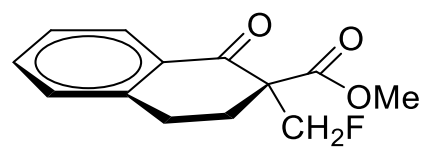

Standard orientation:

\begin{tabular}{rrrrrr} 
Center & Atomic & Atomic & \multicolumn{3}{c}{ Coordinates (Angstroms) } \\
Number & Number & Type & X & Y & Z \\
\hline 1 & 6 & 0 & -3.537924 & 0.580719 & -1.049836 \\
2 & 6 & 0 & -3.895358 & -0.439864 & -0.169072 \\
3 & 6 & 0 & -2.970961 & -0.936890 & 0.738530 \\
4 & 6 & 0 & -1.668260 & -0.437595 & 0.780426 \\
5 & 6 & 0 & -1.315927 & 0.580940 & -0.111617 \\
6 & 6 & 0 & -2.252761 & 1.092289 & -1.013350 \\
7 & 6 & 0 & -0.673011 & -0.987645 & 1.776761 \\
8 & 6 & 0 & 0.517829 & -0.058273 & 2.003309 \\
9 & 6 & 0 & 1.122351 & 0.392613 & 0.665356 \\
10 & 6 & 0 & 0.058221 & 1.165848 & -0.117510 \\
11 & 8 & 0 & 0.308361 & 2.208198 & -0.666641 \\
12 & 6 & 0 & 1.593944 & -0.844885 & -0.098334 \\
13 & 8 & 0 & 1.204218 & -0.837128 & -1.370283 \\
14 & 8 & 0 & 2.255828 & -1.711454 & 0.402202 \\
15 & 6 & 0 & 2.360830 & 1.253354 & 0.889763 \\
16 & 9 & 0 & 3.076241 & 1.360337 & -0.284510 \\
17 & 1 & 0 & -4.262276 & 0.971954 & -1.754840 \\
18 & 1 & 0 & -4.902273 & -0.842150 & -0.185915 \\
& & & 537 & \\
& & 0 & &
\end{tabular}




\begin{tabular}{rrrrrr}
19 & 1 & 0 & -3.261258 & -1.720396 & 1.431984 \\
20 & 1 & 0 & -1.941370 & 1.890779 & -1.676973 \\
21 & 1 & 0 & -1.179249 & -1.184486 & 2.724962 \\
22 & 1 & 0 & -0.304906 & -1.957170 & 1.420997 \\
23 & 1 & 0 & 0.202821 & 0.828454 & 2.563842 \\
24 & 1 & 0 & 1.286787 & -0.569021 & 2.586136 \\
25 & 1 & 0 & 3.008881 & 0.781032 & 1.631929 \\
26 & 1 & 0 & 2.075301 & 2.257267 & 1.208211 \\
27 & 6 & 0 & 1.675571 & -1.928740 & -2.164034 \\
28 & 1 & 0 & 1.326423 & -2.874169 & -1.749223 \\
29 & 1 & 0 & 1.265659 & -1.767611 & -3.156532 \\
30 & 1 & 0 & 2.764774 & -1.925675 & -2.188564 \\
\hline
\end{tabular}

Zero-point correction=

Thermal correction to Energy=

Thermal correction to Enthalpy=

Thermal correction to Gibbs Free Energy=

Sum of electronic and zero-point Energies=

Sum of electronic and thermal Energies=

Sum of electronic and thermal Enthalpies=

Sum of electronic and thermal Free Energies=
0.241694 (Hartree/Particle)

$$
\begin{gathered}
0.256676 \\
0.257620
\end{gathered}
$$

0.198977

$-828.376115$

$-828.361133$

$-828.360189$

$-828.418833$

tBuOH

\begin{tabular}{|c|c|c|c|c|c|}
\hline \multirow{2}{*}{$\begin{array}{l}\text { Center } \\
\text { Number }\end{array}$} & \multirow{2}{*}{$\begin{array}{l}\text { Atomic } \\
\text { Number }\end{array}$} & \multirow{2}{*}{$\begin{array}{l}\text { Atomic } \\
\text { Type }\end{array}$} & \multicolumn{3}{|c|}{ Coordinates (Angstroms) } \\
\hline & & & $\mathrm{X}$ & $\mathrm{Y}$ & $\mathrm{Z}$ \\
\hline 1 & 6 & 0 & 0.684834 & -1.257112 & -0.509489 \\
\hline 2 & 1 & 0 & 1.739682 & -1.268842 & -0.215874 \\
\hline 3 & 1 & 0 & 0.206721 & -2.146371 & -0.094563 \\
\hline 4 & 1 & 0 & 0.642817 & -1.312853 & -1.600006 \\
\hline 5 & 6 & 0 & 0.684751 & 1.257158 & -0.509487 \\
\hline 6 & 1 & 0 & 0.642723 & 1.312903 & -1.600003 \\
\hline 7 & 1 & 0 & 0.206585 & 2.146384 & -0.094551 \\
\hline 8 & 1 & 0 & 1.739600 & 1.268952 & -0.215878 \\
\hline 9 & 6 & 0 & -1.482713 & -0.000047 & -0.351175 \\
\hline 10 & 1 & 0 & -1.969431 & -0.884077 & 0.064863 \\
\hline 11 & 1 & 0 & -1.969495 & 0.883938 & 0.064883 \\
\hline 12 & 1 & 0 & -1.617976 & -0.000040 & -1.434653 \\
\hline 13 & 6 & 0 & -0.006147 & 0.000000 & 0.014728 \\
\hline 14 & 8 & 0 & 0.019366 & -0.000001 & 1.441602 \\
\hline 15 & 1 & 0 & 0.939497 & 0.000024 & 1.725506 \\
\hline
\end{tabular}

Standard orientation: 
Thermal correction to Energy=

0.143521

Thermal correction to Enthalpy=

0.144465

Thermal correction to Gibbs Free Energy=

0.107790

Sum of electronic and zero-point Energies=

$-233.474896$

Sum of electronic and thermal Energies=

$-233.468176$

Sum of electronic and thermal Enthalpies=

$-233.467232$

Sum of electronic and thermal Free Energies=

$-233.503907$

$t \mathrm{BuOLi}$

Standard orientation:

\begin{tabular}{|c|c|c|c|c|c|}
\hline \multirow{2}{*}{$\begin{array}{l}\text { Center } \\
\text { Number }\end{array}$} & \multirow{2}{*}{$\begin{array}{l}\text { Atomic } \\
\text { Number }\end{array}$} & \multirow{2}{*}{$\begin{array}{l}\text { Atomic } \\
\text { Type }\end{array}$} & \multicolumn{3}{|c|}{ Coordinates (Angstroms) } \\
\hline & & & $\mathrm{X}$ & $\mathrm{Y}$ & $\mathrm{Z}$ \\
\hline 1 & 6 & 0 & 0.628499 & 1.416526 & -0.290277 \\
\hline 2 & 1 & 0 & 0.255735 & 1.751164 & -1.262068 \\
\hline 3 & 1 & 0 & 0.255830 & 2.106328 & 0.471857 \\
\hline 4 & 1 & 0 & 1.721517 & 1.467285 & -0.300562 \\
\hline 5 & 6 & 0 & 0.630354 & -0.959441 & -1.080813 \\
\hline 6 & 1 & 0 & 1.723521 & -0.992961 & -1.117740 \\
\hline 7 & 1 & 0 & 0.258176 & -1.968588 & -0.884920 \\
\hline 8 & 1 & 0 & 0.258817 & -0.645669 & -2.060032 \\
\hline 9 & 6 & 0 & 0.628972 & -0.456231 & 1.371626 \\
\hline 10 & 1 & 0 & 0.256692 & 0.217815 & 2.147982 \\
\hline 11 & 1 & 0 & 0.257172 & -1.461098 & 1.589292 \\
\hline 12 & 1 & 0 & 1.722063 & -0.472118 & 1.420492 \\
\hline 13 & 6 & 0 & 0.110350 & 0.000212 & -0.000122 \\
\hline 14 & 8 & 0 & -1.267181 & -0.000696 & -0.000936 \\
\hline 15 & 3 & 0 & -2.853709 & -0.000993 & 0.000235 \\
\hline
\end{tabular}

Zero-point correction $=$

Thermal correction to Energy=

Thermal correction to Enthalpy=

Thermal correction to Gibbs Free Energy=

Sum of electronic and zero-point Energies=

Sum of electronic and thermal Energies=

Sum of electronic and thermal Enthalpies=

Sum of electronic and thermal Free Energies=
0.126895 (Hartree/Particle)

$$
0.134442
$$

0.135386

0.096396

$-240.445769$

$-240.438221$

$-240.437277$

$-240.476267$

\section{${ }^{+} \mathrm{CH}_{2} \mathrm{~F}$}

Standard orientation:

\begin{tabular}{lccccc} 
Center & Atomic & Atomic & Coordinates (Angstroms) & \\
Number & Number & Type & X & $Y$ & Z \\
& & & \multicolumn{2}{c}{ s39 } &
\end{tabular}




$\begin{array}{rlrrrr}1 & 6 & 0 & -0.000036 & 0.590701 & 0.000000 \\ 2 & 1 & 0 & 0.000274 & 1.084276 & 0.975815 \\ 3 & 1 & 0 & 0.000274 & 1.084276 & -0.975815 \\ 4 & 9 & 0 & -0.000036 & -0.634751 & 0.000000\end{array}$

Zero-point correction $=$

Thermal correction to Energy=

Thermal correction to Enthalpy=

Thermal correction to Gibbs Free Energy=

Sum of electronic and zero-point Energies=

Sum of electronic and thermal Energies=

Sum of electronic and thermal Enthalpies=

Sum of electronic and thermal Free Energies=

0.027819 (Hartree/Particle)
0.030684
0.031628
0.005921
-138.692342
-138.689477
-138.688533
-138.714241

0.027819 (Hartree/Particle)

\author{
.
}

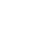

$-138.688533$

$-138.714241$

\section{$I^{-}$}

Standard orientation:

\begin{tabular}{|c|c|c|c|c|c|}
\hline \multirow{2}{*}{$\begin{array}{l}\text { Center } \\
\text { Number }\end{array}$} & \multirow{2}{*}{$\begin{array}{l}\text { Atomic } \\
\text { Number }\end{array}$} & \multirow{2}{*}{$\begin{array}{l}\text { Atomic } \\
\text { Type }\end{array}$} & \multicolumn{3}{|c|}{ Coordinates (Angstroms) } \\
\hline & & & $\mathrm{X}$ & $\mathrm{Y}$ & \\
\hline 1 & 53 & 0 & 0.000000 & 0.000000 & 0.000 \\
\hline
\end{tabular}

Zero-point correction $=$

Thermal correction to Energy=

Thermal correction to Enthalpy=

Thermal correction to Gibbs Free Energy=

Sum of electronic and zero-point Energies=

Sum of electronic and thermal Energies=

Sum of electronic and thermal Enthalpies=

Sum of electronic and thermal Free Energies=

\author{
0.000000 (Hartree/Particle) \\ 0.001416 \\ 0.002360 \\ $-0.016848$ \\ $-6920.012288$ \\ $-6920.010872$ \\ $-6920.009928$ \\ $-6920.029136$
}

Lil

Standard orientation:

\begin{tabular}{cccccc} 
Center & Atomic & Atomic & \multicolumn{3}{c}{ Coordinates (Angstroms) } \\
Number & Number & Type & $X$ & $Y$ & $Z$ \\
-1 & 3 & 0 & 0.000000 & 0.000000 & -2.273481 \\
2 & 53 & 0 & 0.000000 & 0.000000 & 0.128688
\end{tabular}

Zero-point correction $=$

Thermal correction to Energy=

Thermal correction to Enthalpy=
0.001141 (Hartree/Particle)

0.003725

0.004669 
Thermal correction to Gibbs Free Energy=

Sum of electronic and zero-point Energies=

Sum of electronic and thermal Energies=

Sum of electronic and thermal Enthalpies=

Sum of electronic and thermal Free Energies=
$-0.021685$

$-6927.514932$

$-6927.512348$

$-6927.511404$

$-6927.537757$

\section{References}

1. Ko, T. Y.; Youn, S. W. Adv. Synth. Catal. 2016, 358, 1934.

2. Saha, A. K.; Das, S.; Mukherjee, D.; Fronczek, F. R. Tetrahedron Lett. 1994, 35, 3353.

3. Geibel, I.; Christoffers, J. Eur. J. Org. Chem. 2016. $2016,918$.

4. Civicos, J. F.; Coimbra, J. S. M.; Costa, P. R. R. Synthesis. 2017, 49, 3998.

5. Qiu, J.; Wang, Y.; Qi, G.; Karmaker, P. G.; Yin, H.; Chen, F. Chem. Eur. J. 2017, 23, 1775.

6. Kobayashi, S.; Gustafsson, T.; Shimizu, Y.; Kiyohara, H.; Matsubara, R. Org. Lett. 2006, 8, 4923.

7. Vita, M. V.; Waser, J. Org. Lett. 2013, 15, 3246.

8. Companys, S.; Peixoto, P. A.; Bosset, C.; Chassaing, S.; Miqueu, K.; Sotiropoulos, J. M.; Pouysegu, L.; Quideau, S. Chem. Eur. J. 2017, 23, 13309.

9. Baud, L. G.; Manning, M. A.; Arkless, H. L.; Stephens, T. C.; Unsworth, W. P. Chem. Eur. J. 2017, $23,2225$.

10. Gu, X.; Zhang, Y.; Xu, Z.; Che. C. Chem. Commun. 2014, 50, 7870.

11. Craig, R. A.; Loskot, S. A.; Mohr, J. T.; Behenna, D. C.; Harned, A. M.; Stoltz, B. M. Org. Lett. 2015, 17, 5160 .

12. Rambla, M.; Duroure, L.; Chabaud, L.; Guillou, C. Eur. J. Org. Chem. 2014. 2014, 7716.

13. Choudhury, A. R.; Manna, M. S.; Mukherjee, S. Chem. Sci. 2017, 8, 6686.

14. Kenny, M.; Christensen, J.; Coles, S. J.; Franckevicius, V. Org. Lett. 2015, 17, 3926.

15. Bowen, J. G.; Hockley, M. H.; Housley, J. R.; Hunneyball, I. M.; Titman, R. B.; Webber, D. G. Eur. Pat. Appl. 1990, EP 354694 A1 19900214.

16. Fernandez, G. D.; Brand, J. P.; Waser, J. Chem. Eur. J. 2010, 16, 9457.

17. Liu, Y.; Lu, L.; Shen, Q. Angew. Chem., Int. Ed. 2017, 56, 9930.

18. Frisch, M. J.; Trucks, G. W.; Schlegel, H. B.; Scuseria, G. E.; Robb, M. A.; Cheeseman, J. R.; Scalmani, G.; Barone, V.; Mennucci, B.; Petersson, G. A.; Nakatsuji, H.; Caricato, M.; Li, X.; Hratchian, H. P.; Izmaylov, A. F.; Bloino, J.; Zheng, G.; Sonnenberg, J. L.; Hada, M.; Ehara, M.; Toyota, K.; Fukuda, R.; Hasegawa, J.; Ishida, M.; Nakajima, T.; Honda, Y.; Kitao, O.; Nakai, H.; Vreven, T.; Montgomery, J. A., Jr.; Peralta, J. E.; Ogliaro, F.; Bearpark, M.; Heyd, J. J.; Brothers, E.; Kudin, K. N.; Staroverov, V. N.; Kobayashi, R.; Normand, J.; Raghavachari, K.; Rendell, A.; Burant, J. C.; Iyengar, S. S.; Tomasi, J.; Cossi, M.; Rega, N.; Millam, J. M.; Klene, M.; Knox, J. E.; Cross, J. B.; Bakken, V.; Adamo, C.; Jaramillo, J.; Gomperts, R.; Stratmann, R. E.; Yazyev, O.; Austin, A. J.; Cammi, R.; Pomelli, C.; Ochterski, J. W.; Martin, R. L.; Morokuma, K.; Zakrzewski, V. G.; Voth, G. A.; Salvador, P.; Dannenberg, J. J.; Dapprich, S.; Daniels, A. D.; Farkas, O.; Foresman, J. B.; Ortiz, J. V.; Cioslowski, J.; Fox, D. J. Gaussian 09, revision D.01; Gaussian, Inc., Wallingford, CT, 2013.

19. (a) Zhao, Y.; Truhlar, D. G. Acc. Chem. Res. 2008, 41, 157.; (b) Zhao, Y.; Truhlar, D. G. Chem. Phys. Lett. 2011, 502, 1. 


\section{NMR spectra}
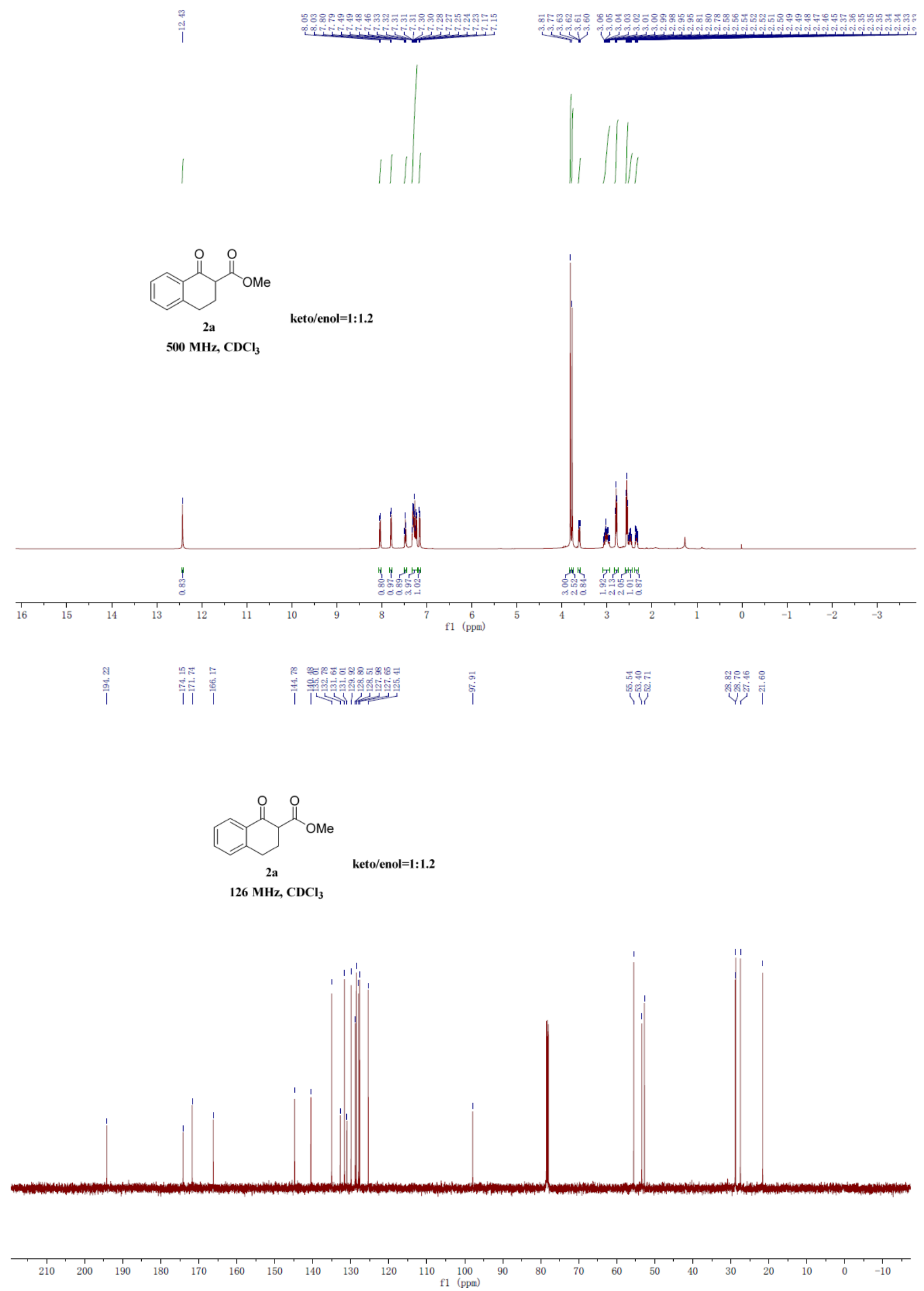


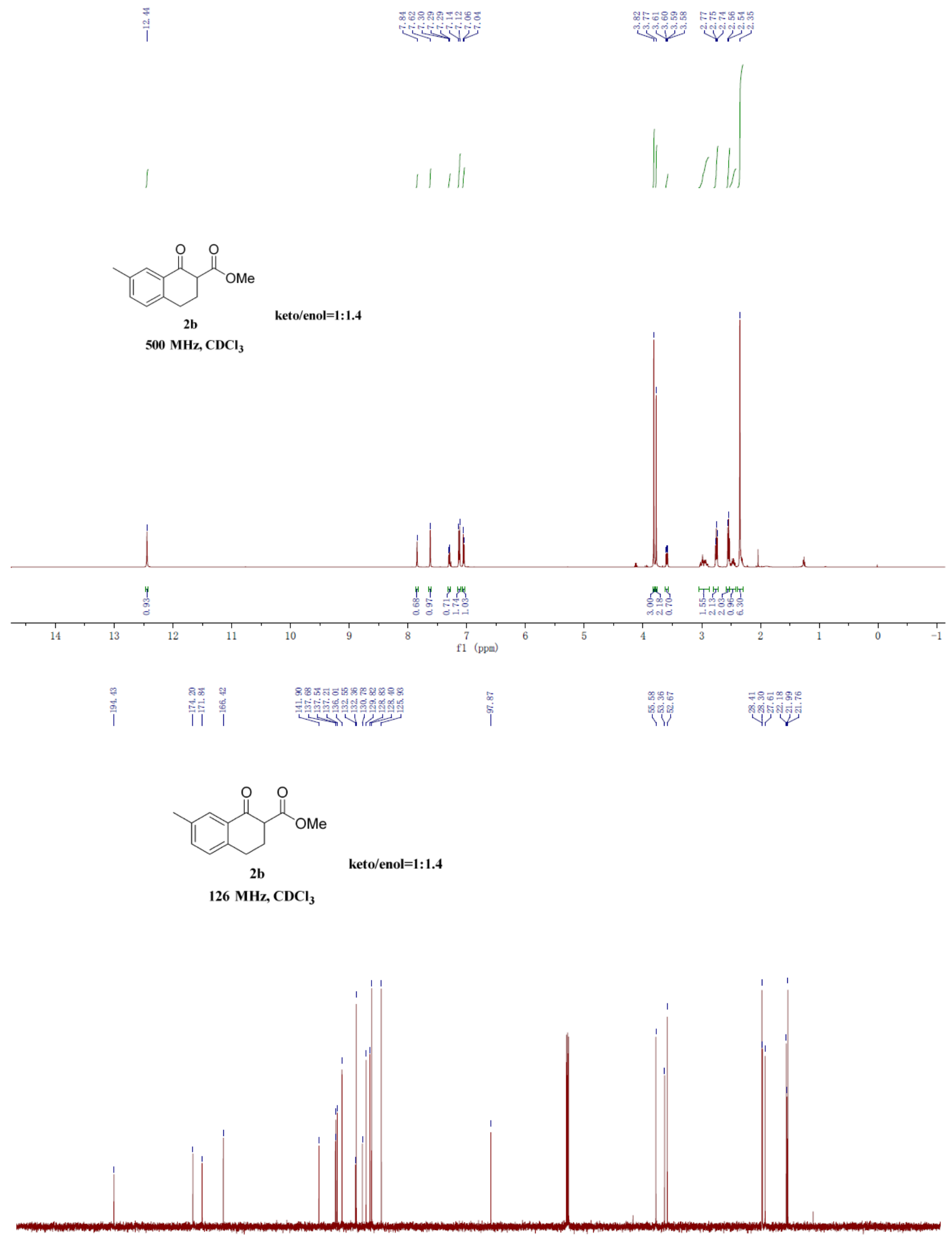

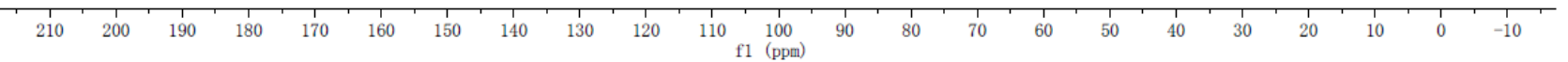



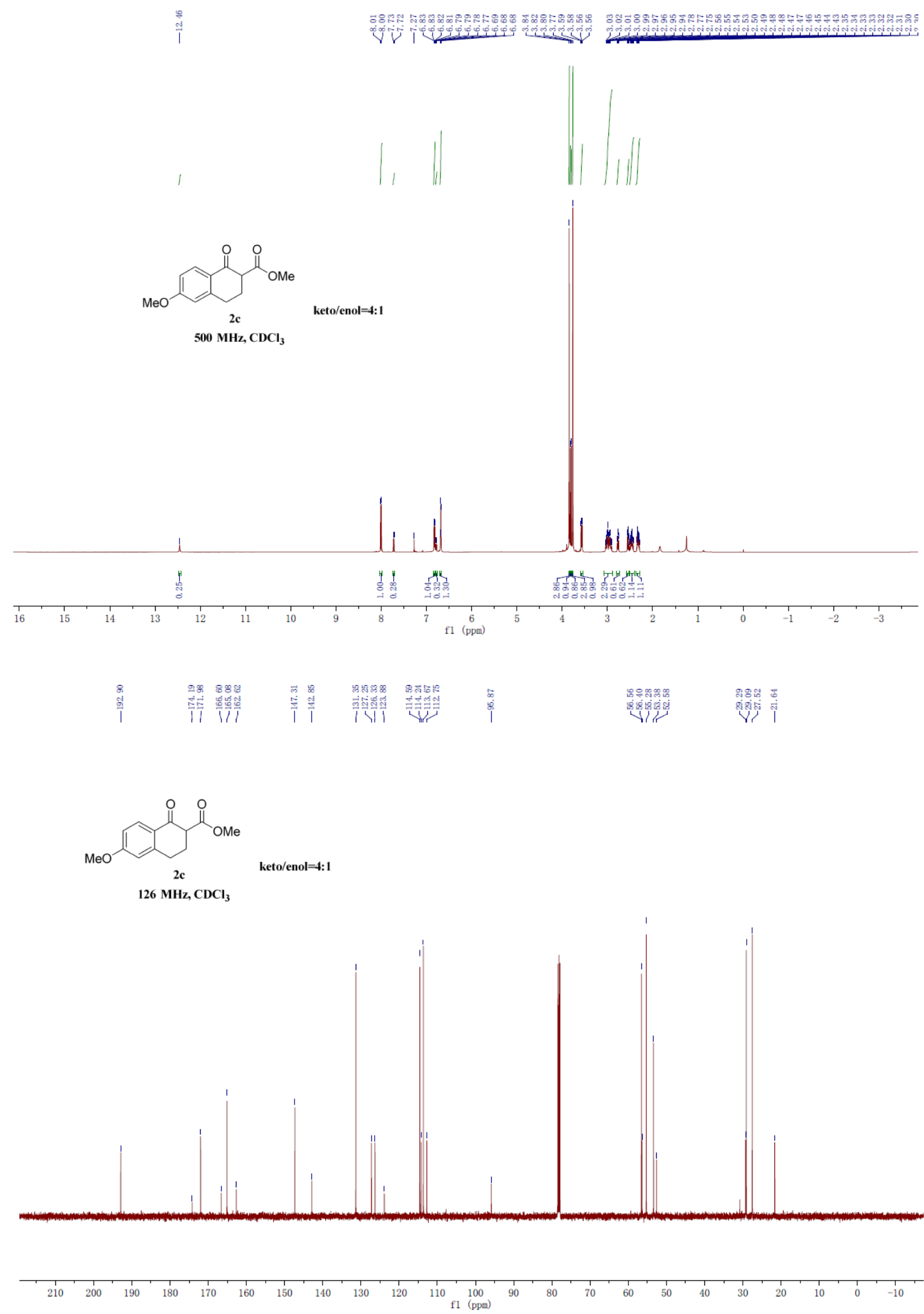


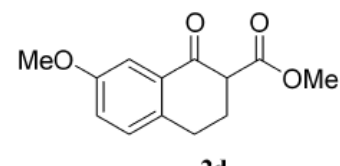

keto/enol=1.2:1

$500 \mathrm{MHz}, \mathrm{CDCl}_{3}$

(1.2:1

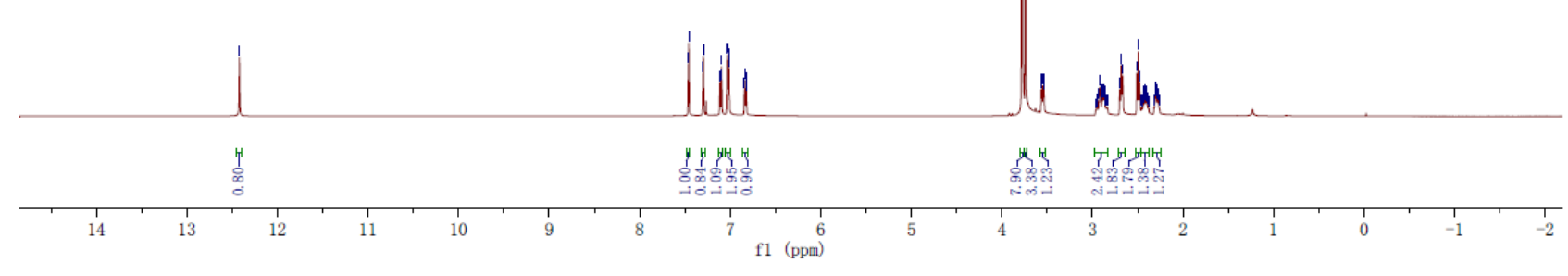

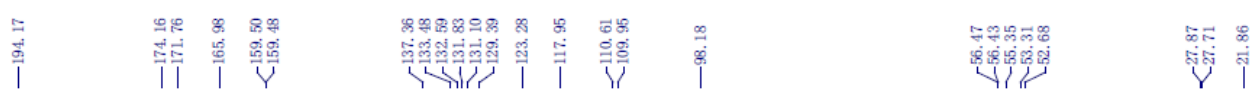

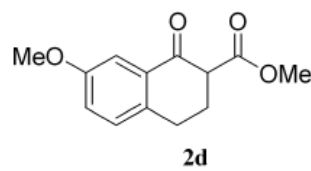

keto/enol=1.2:1

$126 \mathrm{MHz}, \mathrm{CDCl}_{3}$

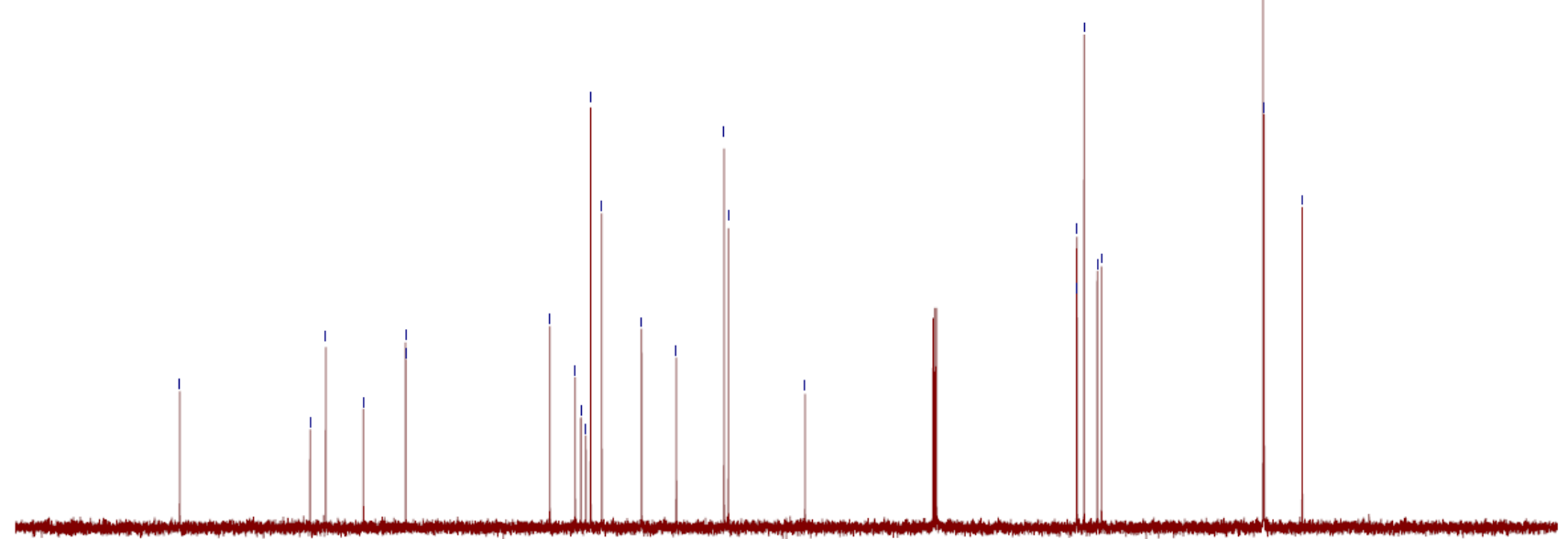

$\begin{array}{llllllllllll}210 & 200 & 190 & 180 & 170 & 160 & 150 & 140 & 130 & 120 & 110 & 100 \\ \mathrm{fl} 1 & (\mathrm{ppm})\end{array}$ 

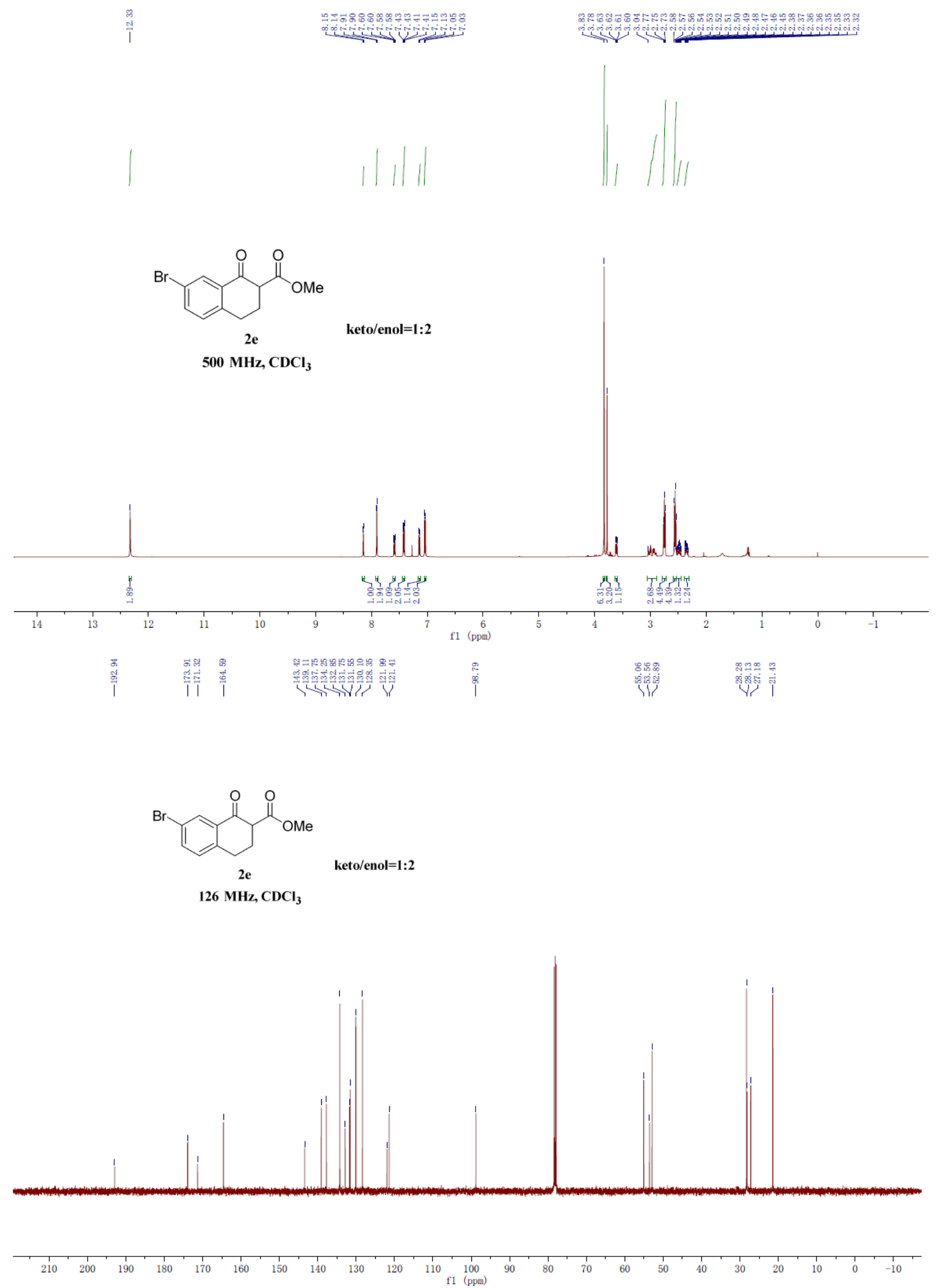

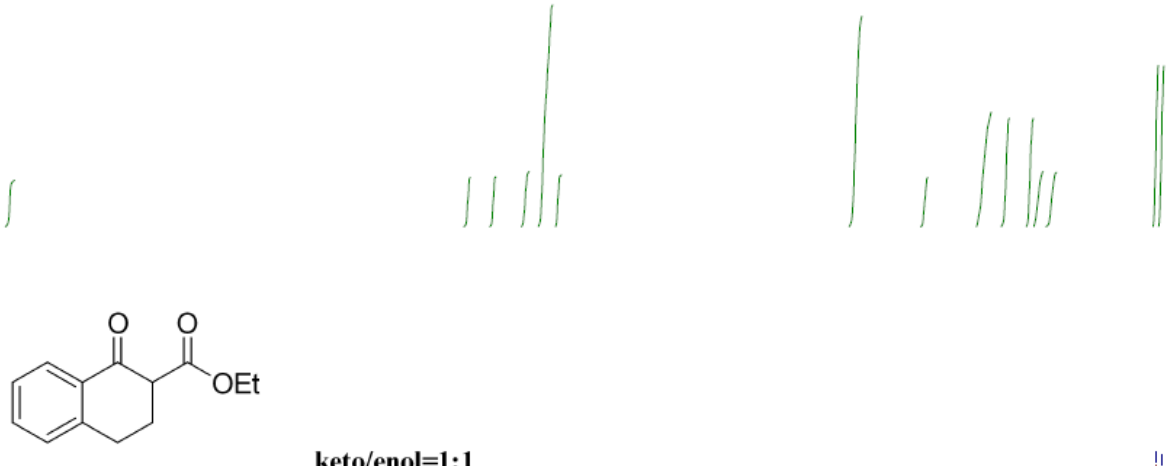

keto/enol=1: 1

$500 \mathrm{MHz}, \mathrm{CDCl}_{3}$
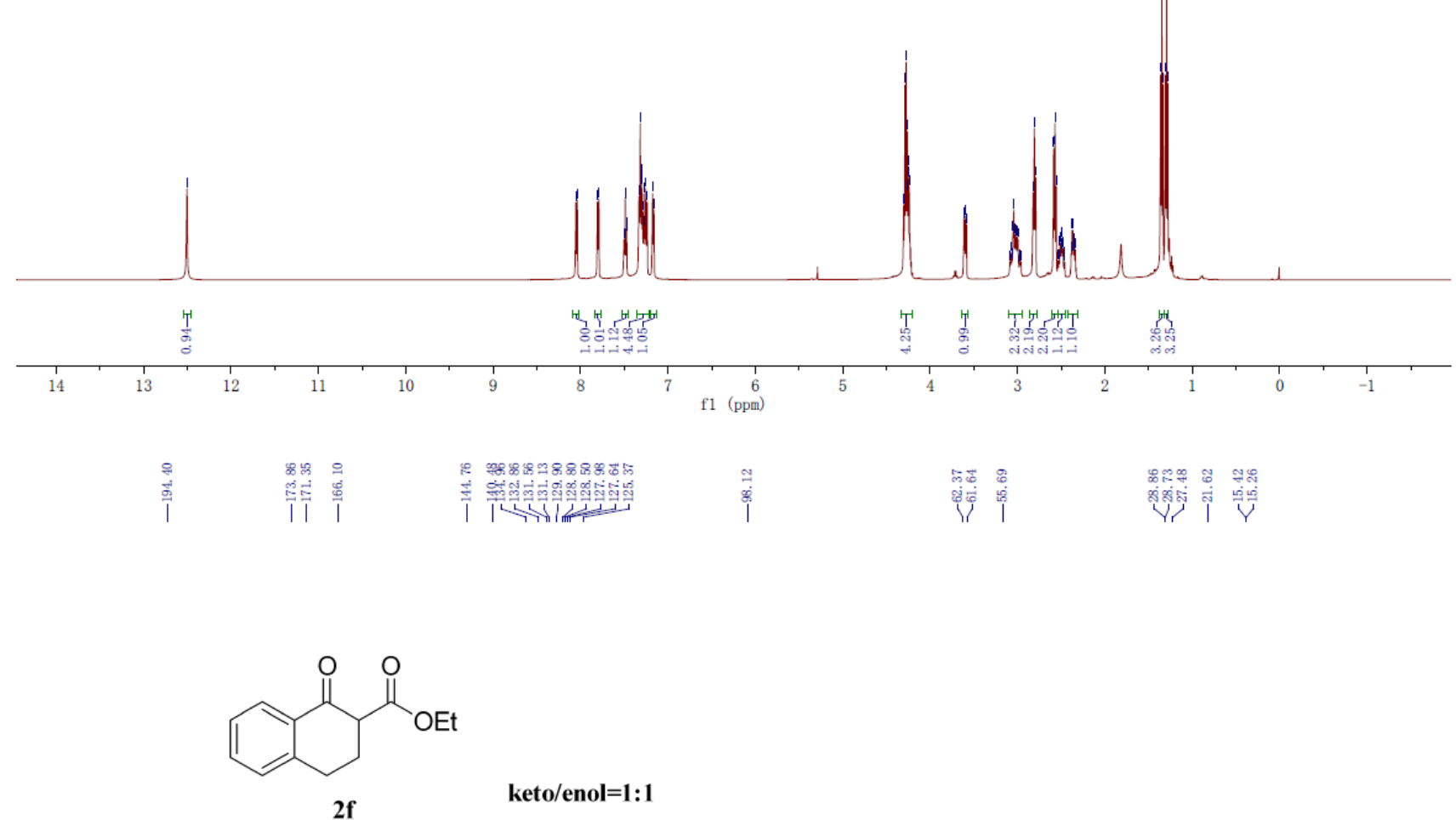

keto/enol=1:1

$126 \mathrm{MHz}, \mathrm{CDCl}_{3}$

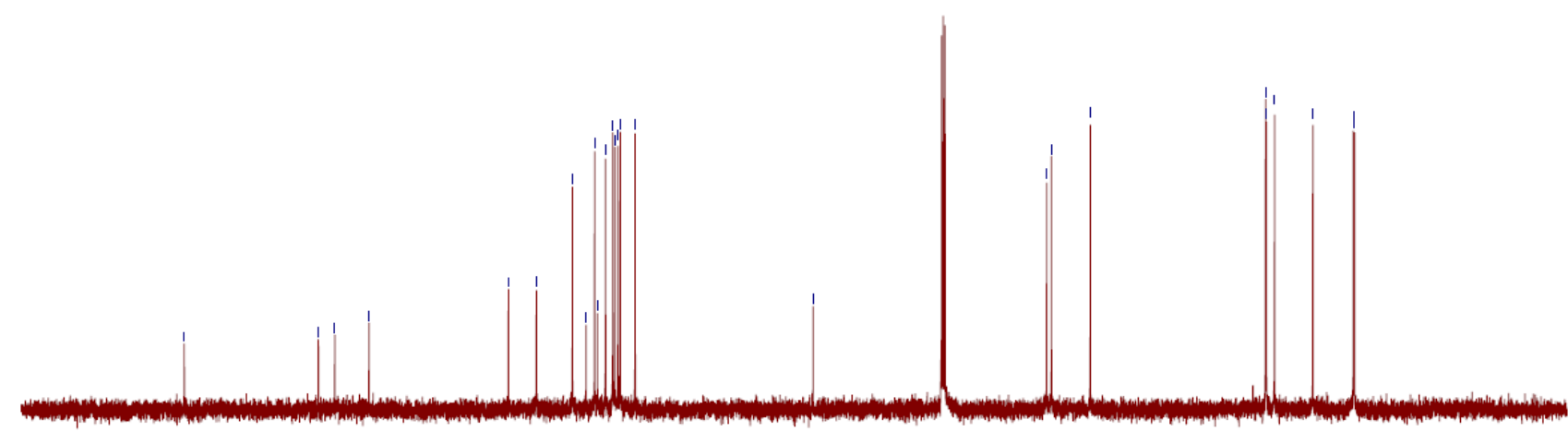

$\begin{array}{llllllllllll}210 & 200 & 190 & 180 & 170 & 160 & 150 & 140 & 130 & 120 & 110 & 100 \\ 1 & 1 & 1 & 1 & 1 & 1\end{array}$ 

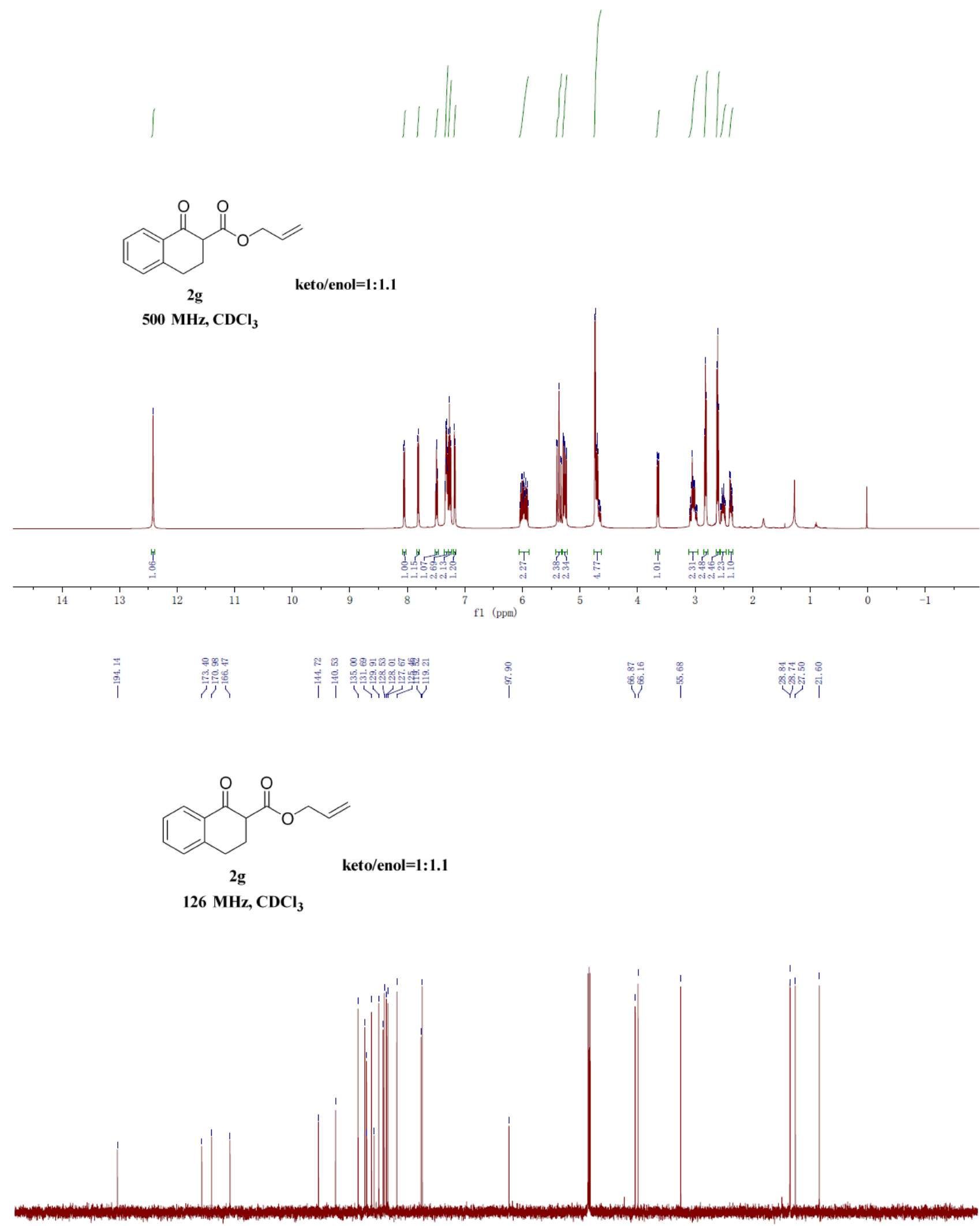

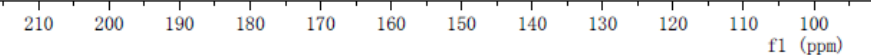




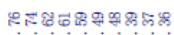

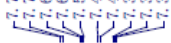
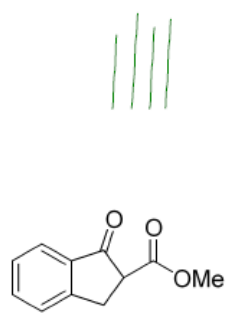

2h

keto/enol=4.5: 1

$500 \mathrm{MHz} \mathrm{CDCl}_{3}$

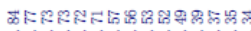

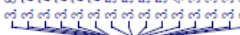

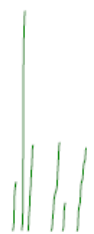

111

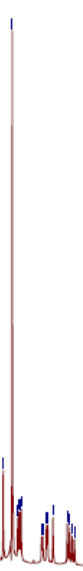

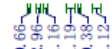

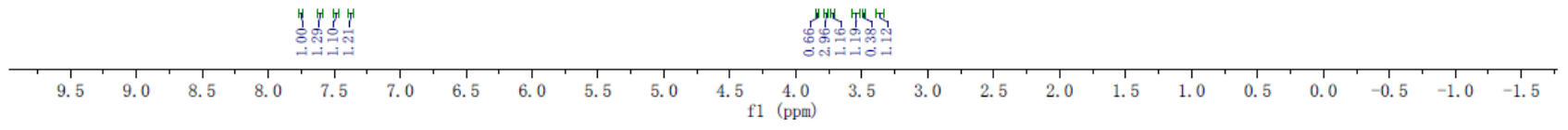

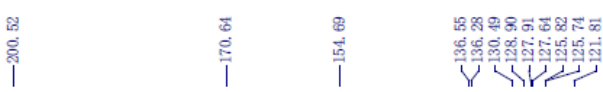

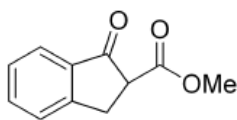

2h keto/enol=4.5: 1

$126 \mathrm{MHz} \mathrm{CDCl}_{3}$

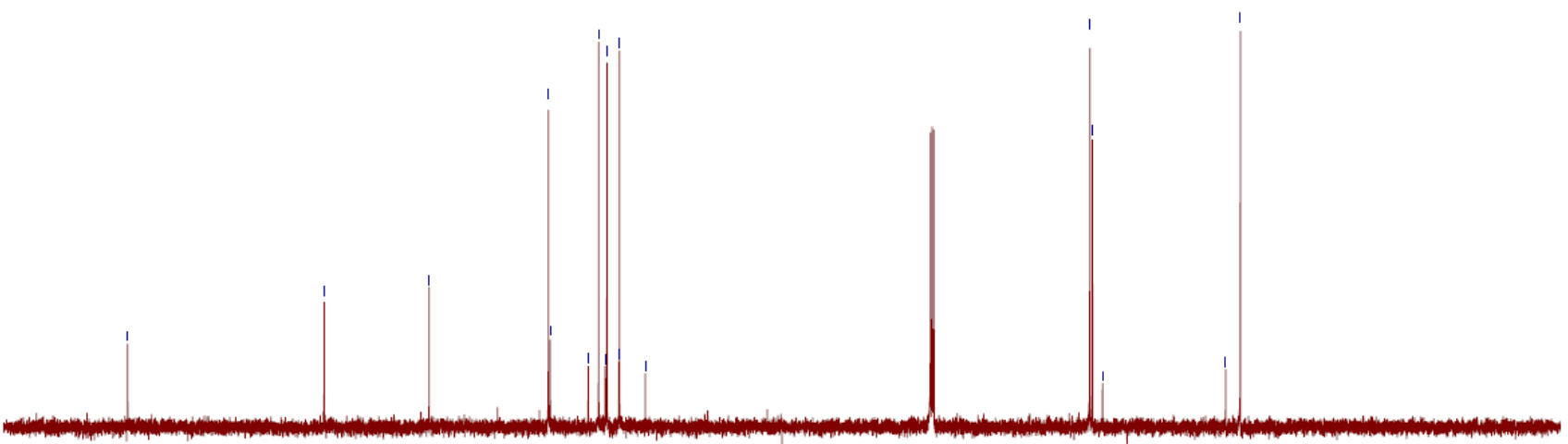

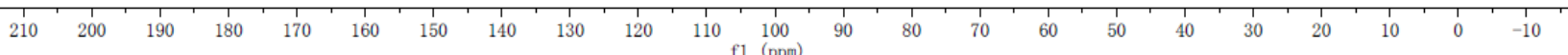




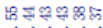

initis
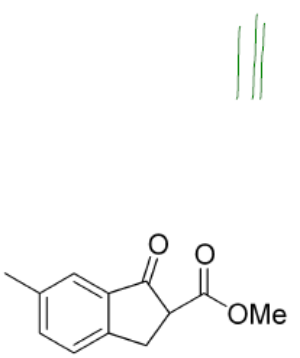

$2 \mathrm{i}$

keto/enol=8: 1

$500 \mathrm{MHz} \mathrm{CDCl}_{3}$

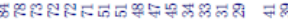

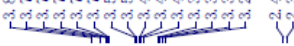
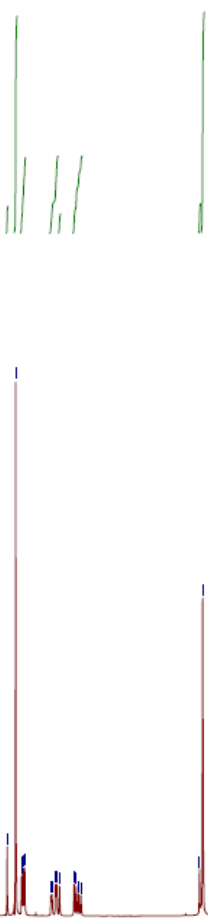

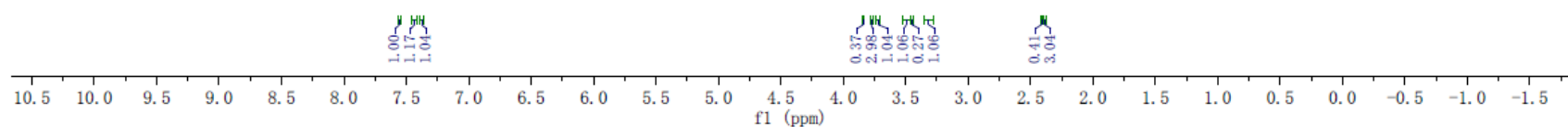

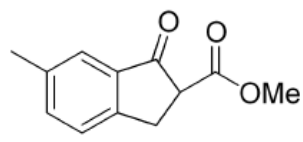

2i

keto/enol=8: 1

$126 \mathrm{MHz}^{\mathrm{CDCl}} \mathrm{Cl}_{3}$

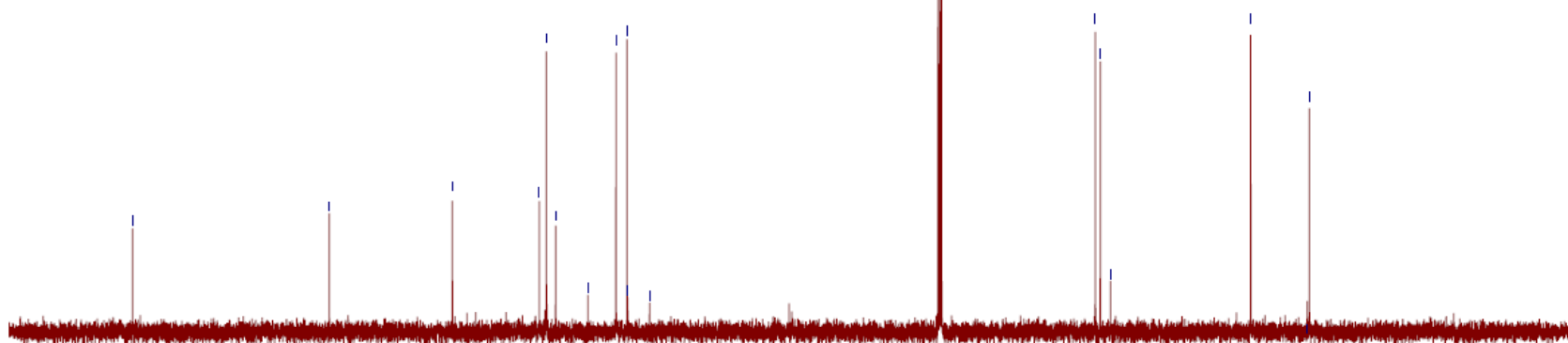

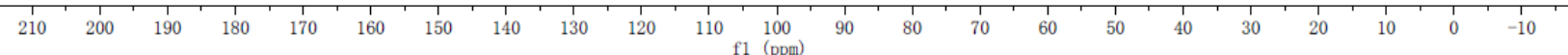




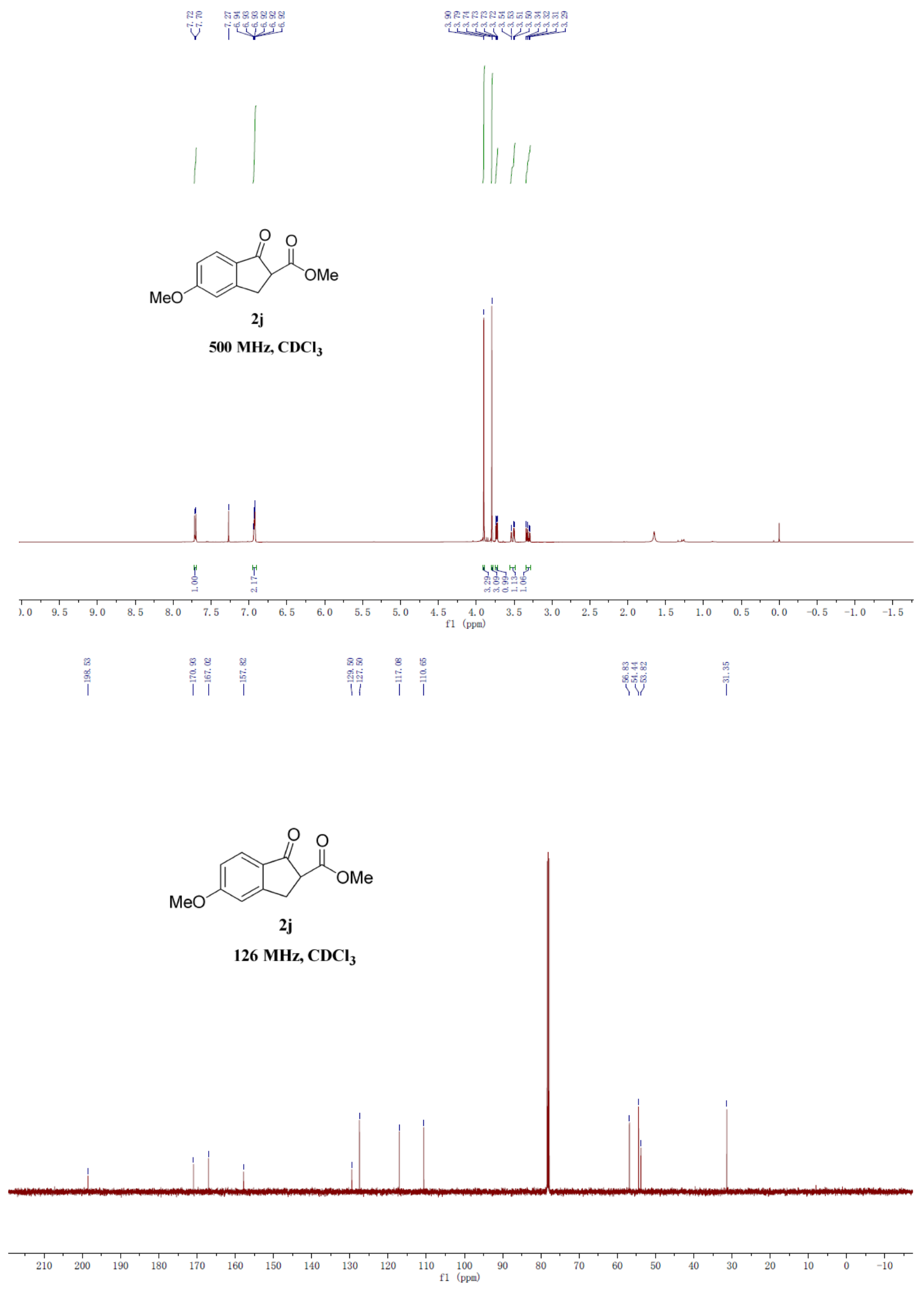



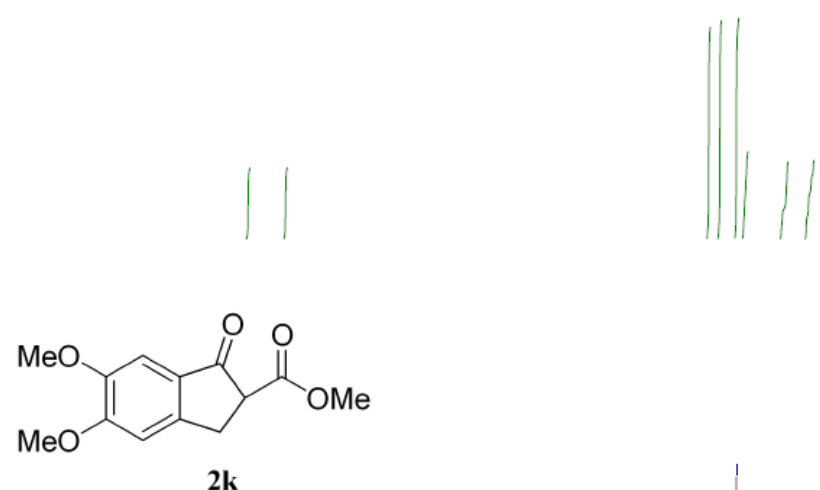

$500 \mathrm{MHz}, \mathrm{CDCl}_{3}$
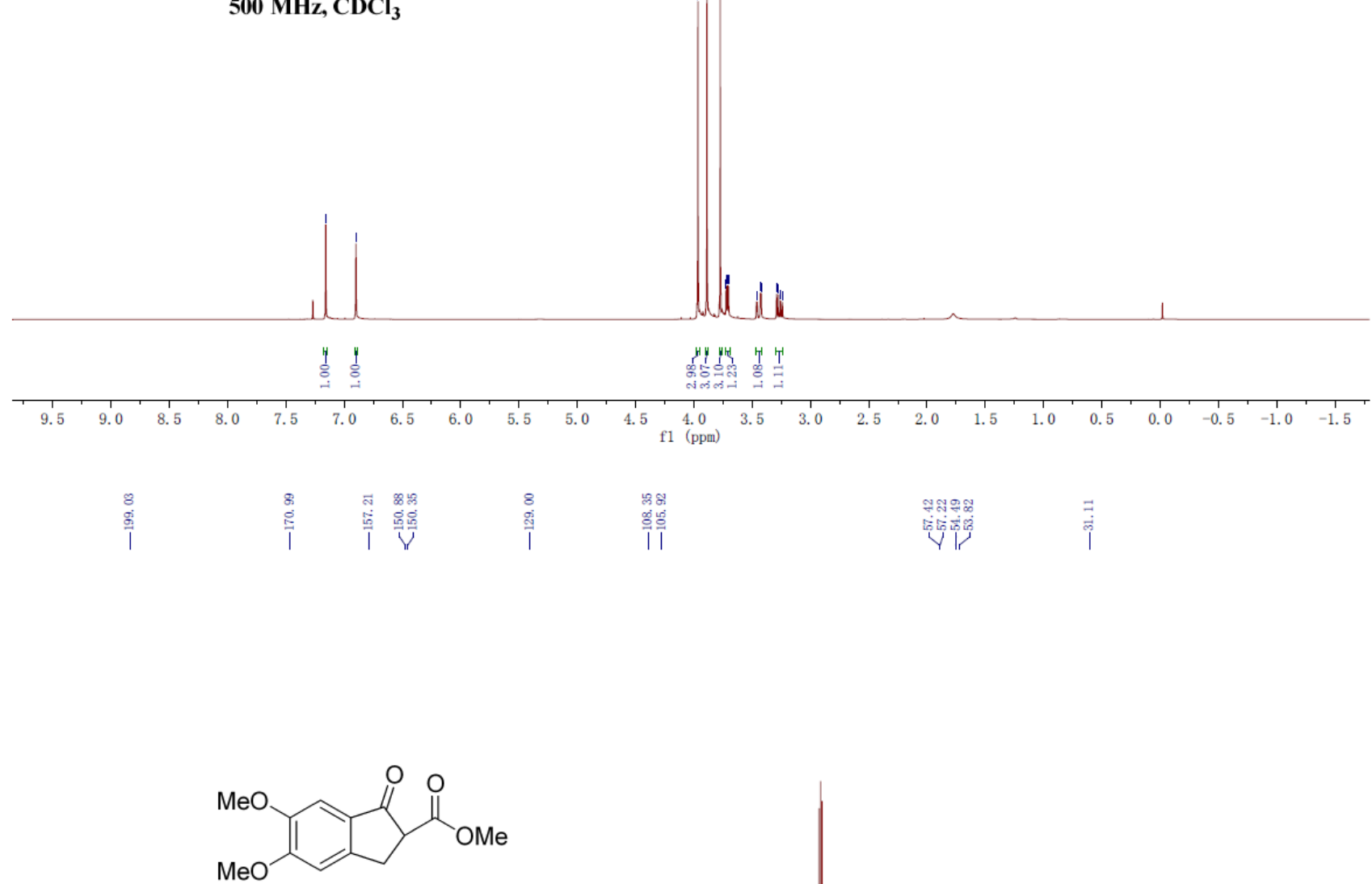

$2 k$

$126 \mathrm{MHz}, \mathrm{CDCl}_{3}$

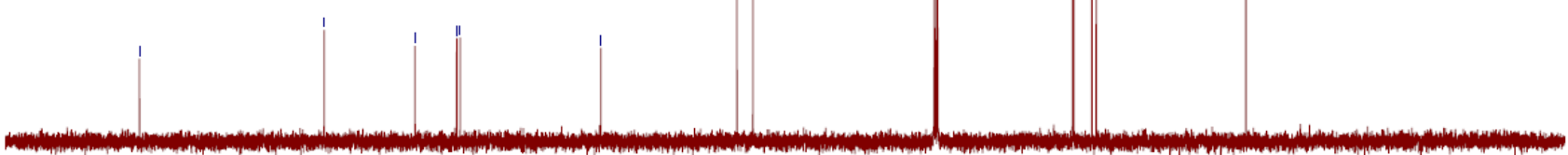

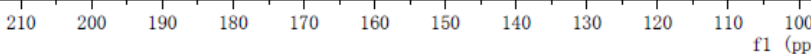



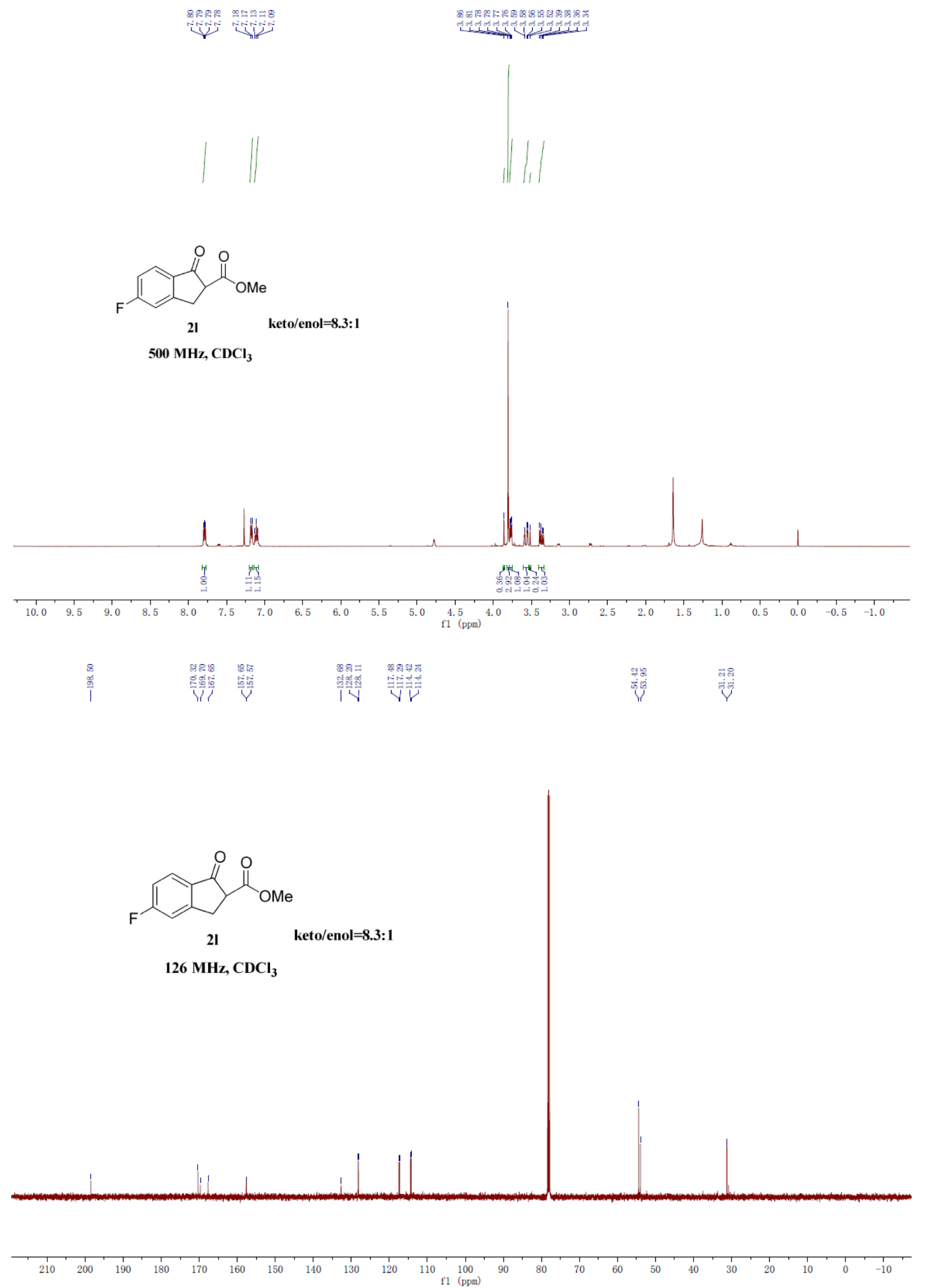

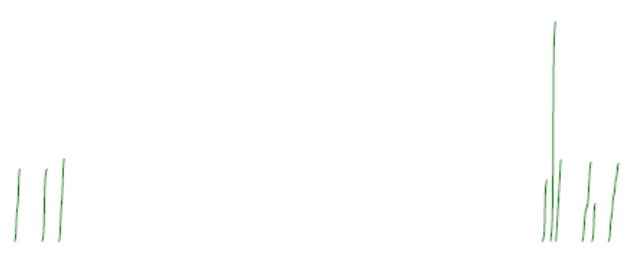<smiles>COC(=O)C1Cc2cc(Cl)ccc2C1=O</smiles>

keto/enol=3.5:

$500 \mathrm{MHz}, \mathrm{CDCl}_{3}$
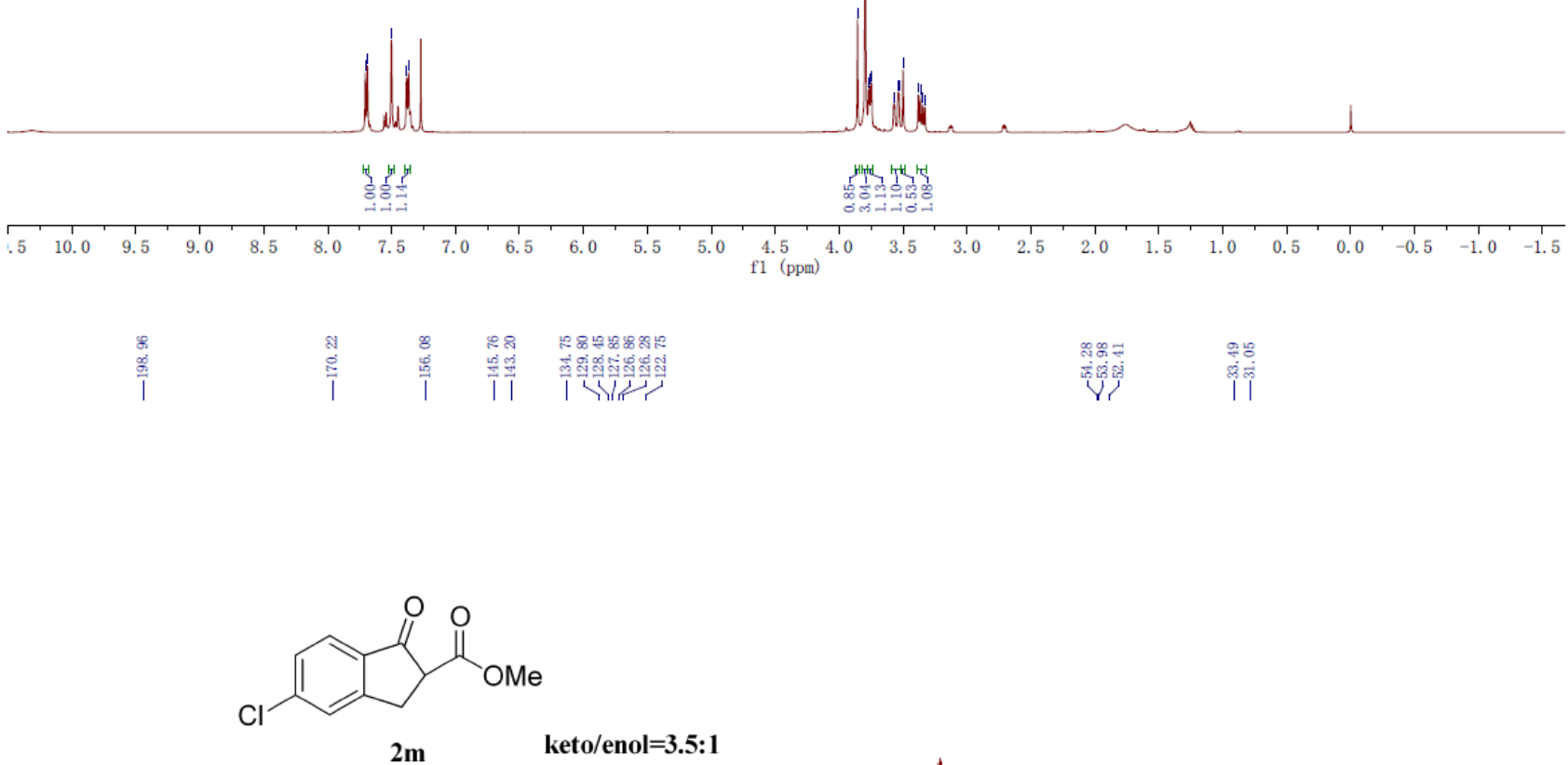

$126 \mathrm{MHz}, \mathrm{CDCl}_{3}$

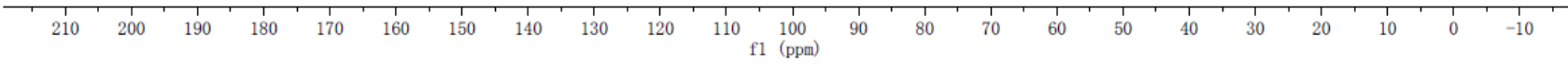




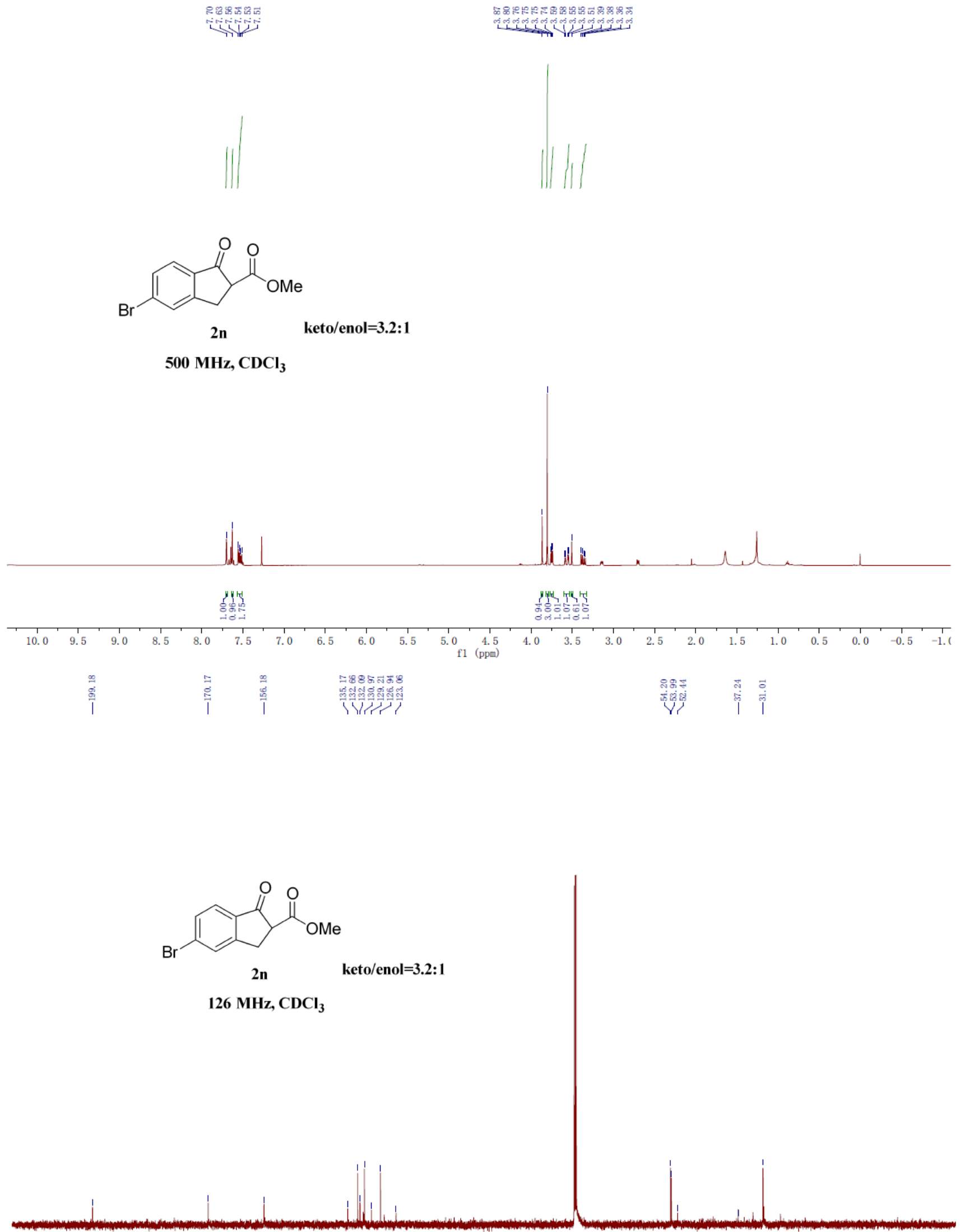

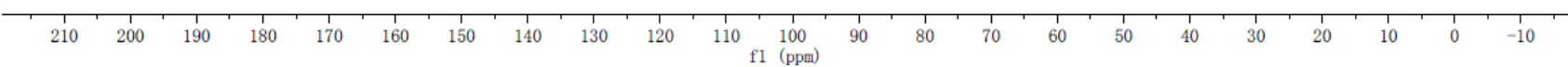



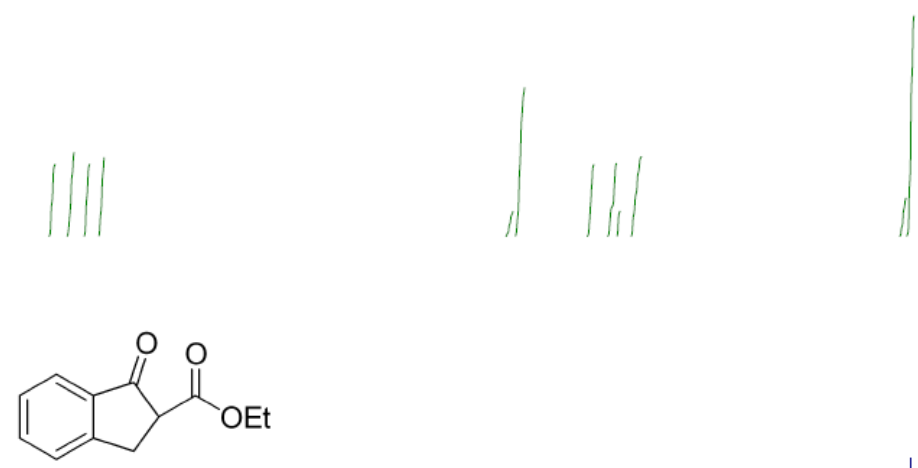

20

keto/enol $=5.7: 1$

$500 \mathrm{MHz} \mathrm{CDCl}_{3}$
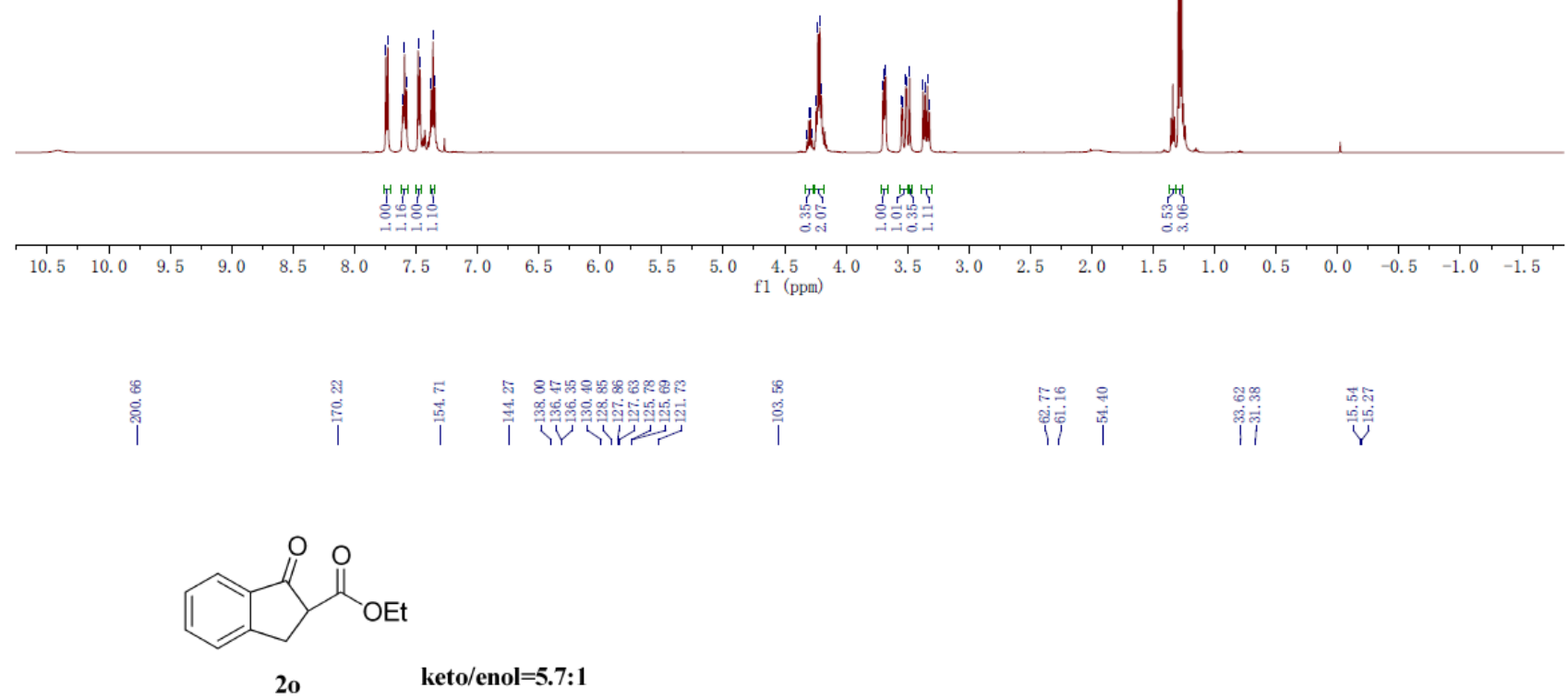

$126 \mathrm{MHz} \mathrm{CDCl}_{3}$
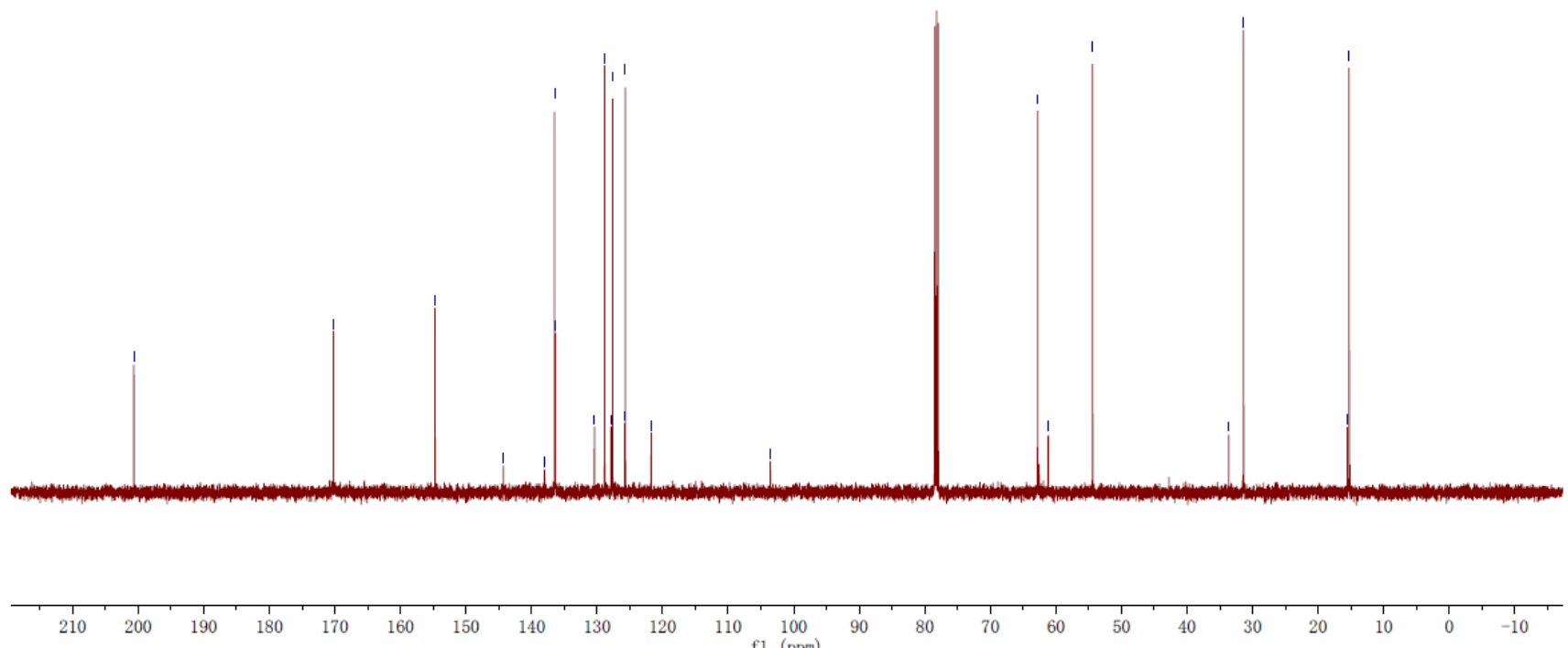


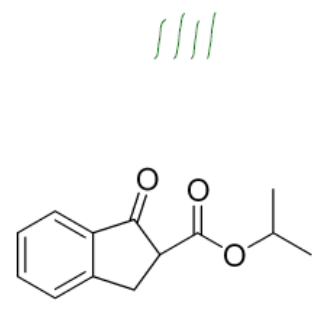

2p

keto/enol=5.8: 1

$500 \mathrm{MHz} \mathrm{CDCl}_{3}$
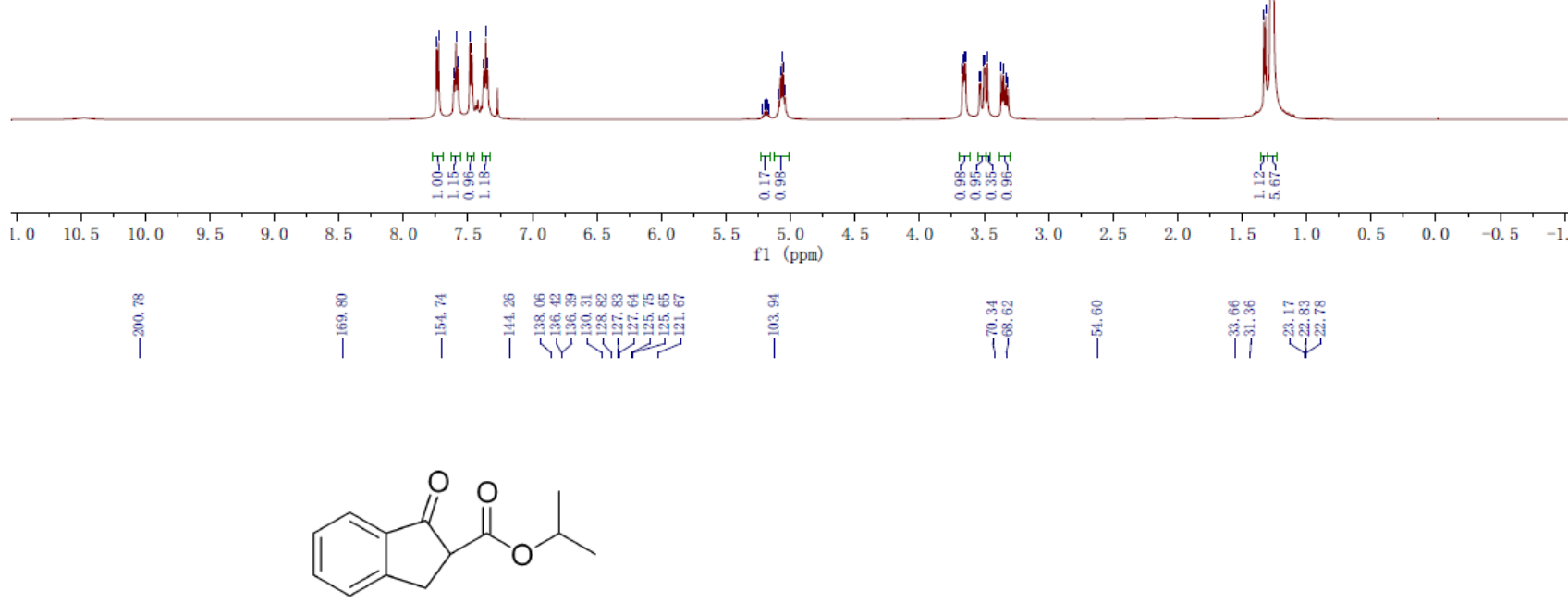

$2 p$

keto/enol=5.8: 1

$126 \mathrm{MHz} \mathrm{CDCl}_{3}$
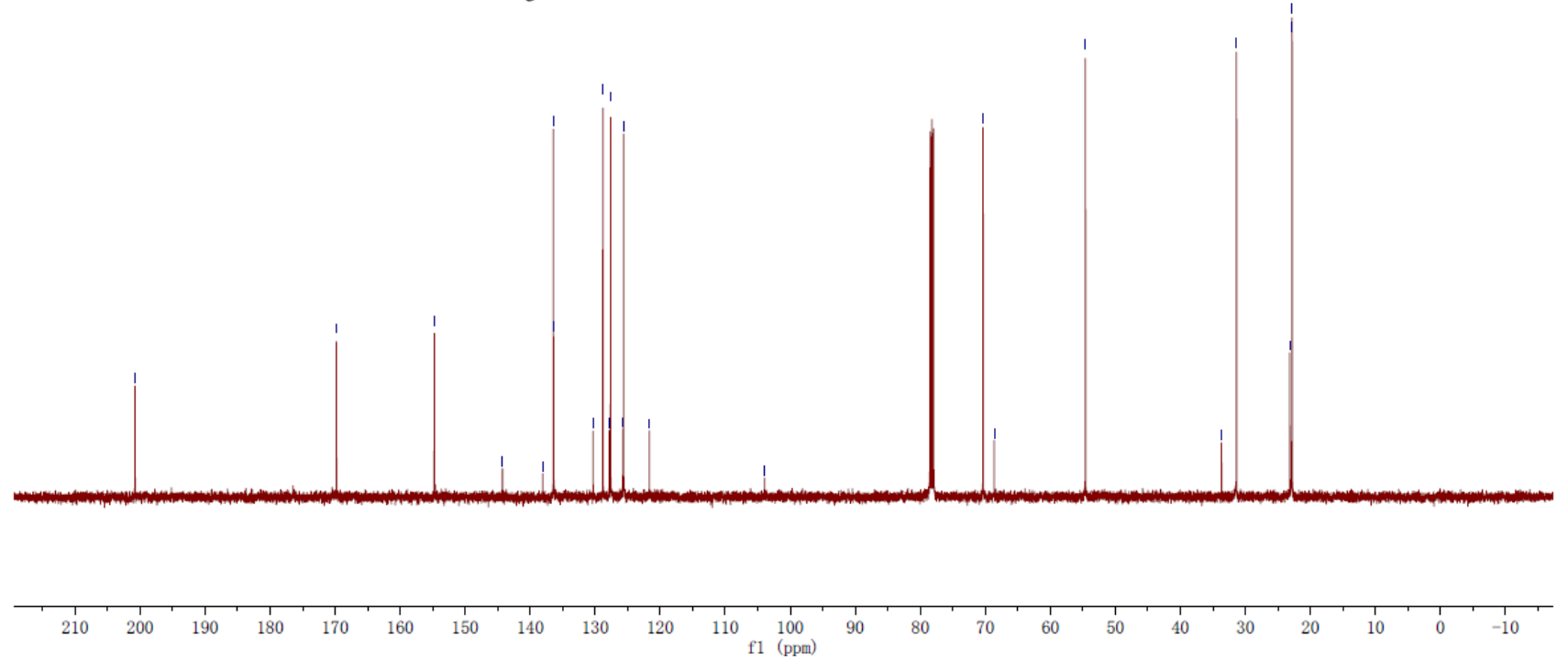
<smiles>CC(C)(C)OC(=O)C1Cc2ccccc2C1=O</smiles>

$2 q$

\section{keto/enol=8.5: 1}

$500 \mathrm{MHz}, \mathrm{CDCl}_{3}$
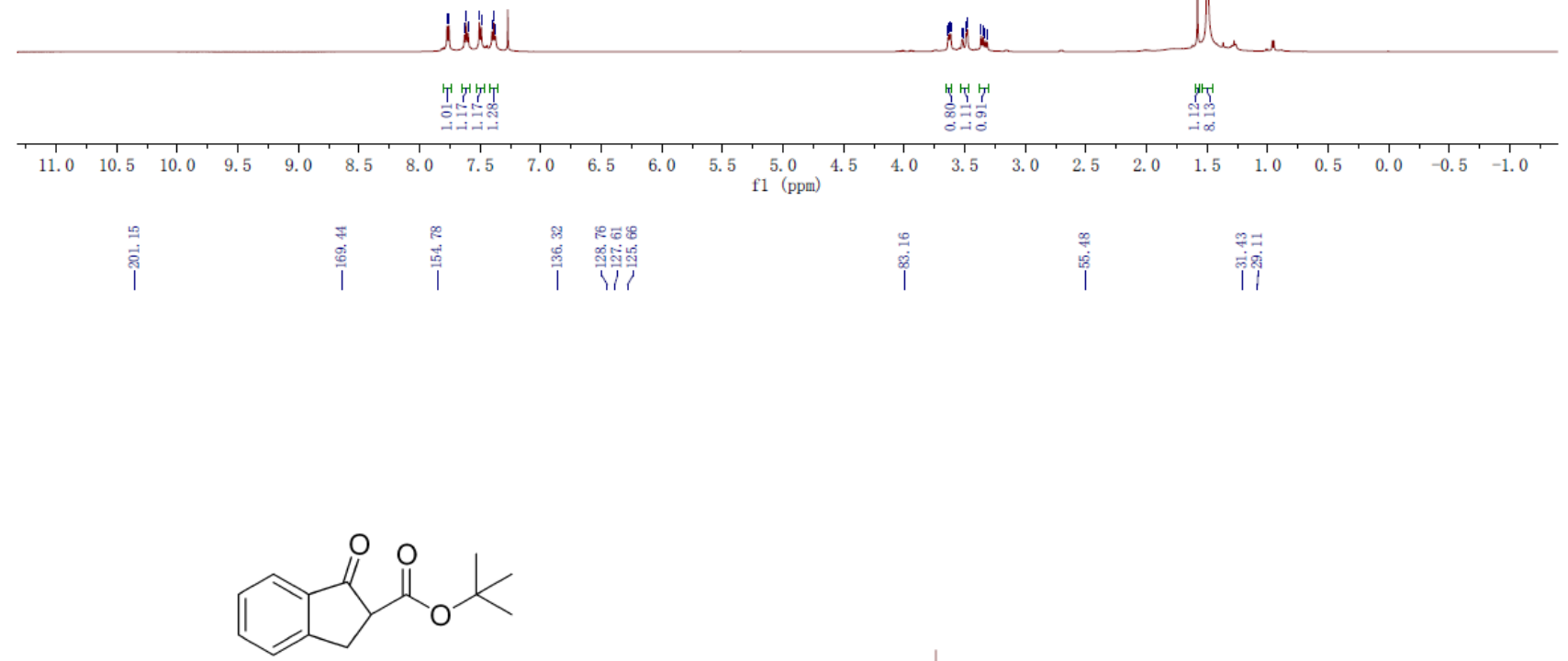

$2 q$

keto/enol=8.5: 1

$126 \mathrm{MHz} \mathrm{CDCl}_{3}$

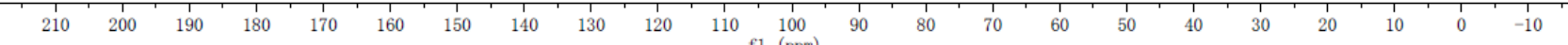


$\stackrel{3}{i}$

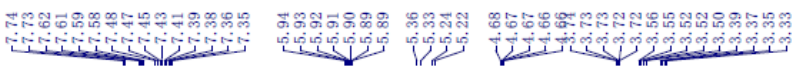
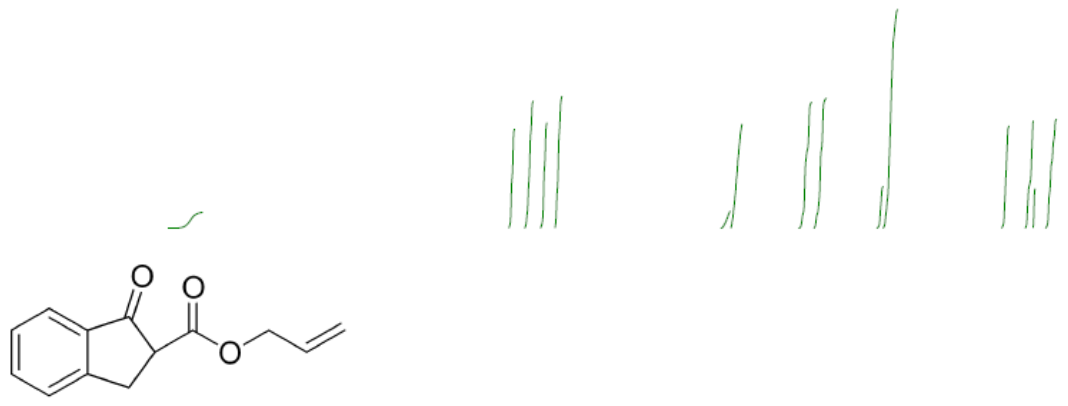

$2 \mathbf{r}$

keto/enol=5.9: 1

$500 \mathrm{MHz}, \mathrm{CDCl}_{3}$

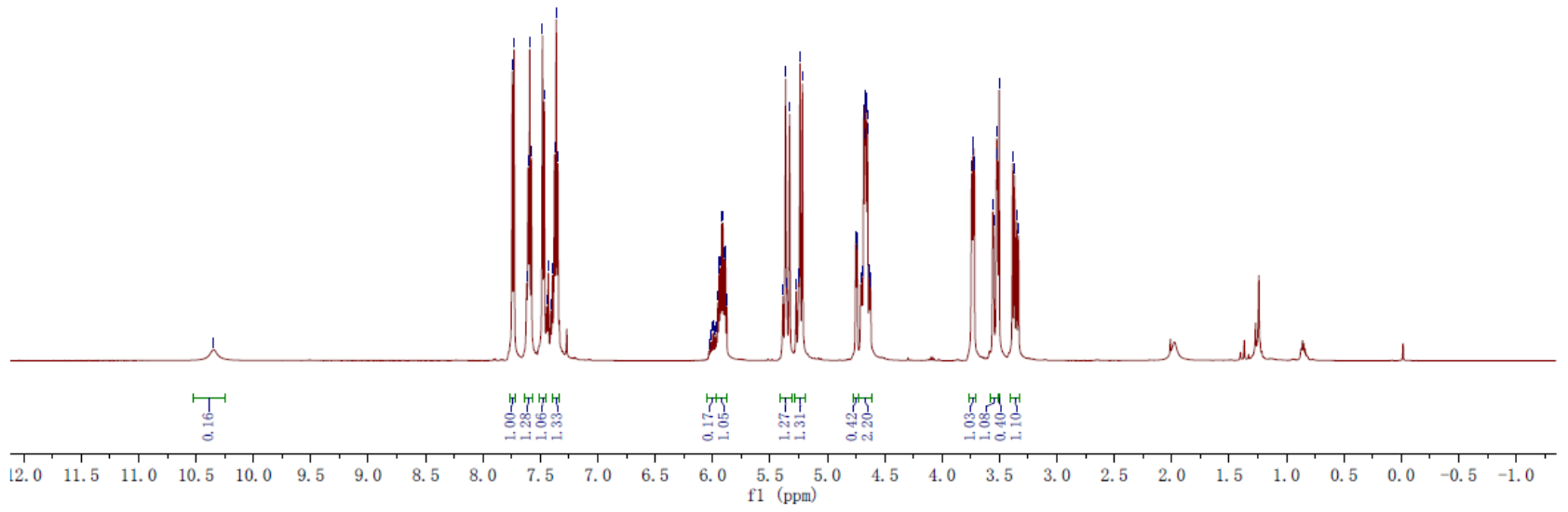

\#

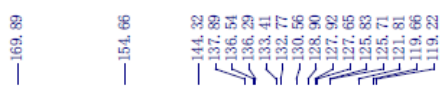

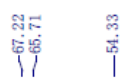

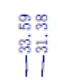

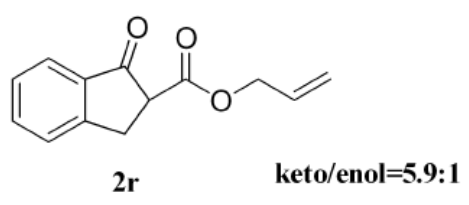

$126 \mathrm{MHz}, \mathrm{CDCl}_{3}$
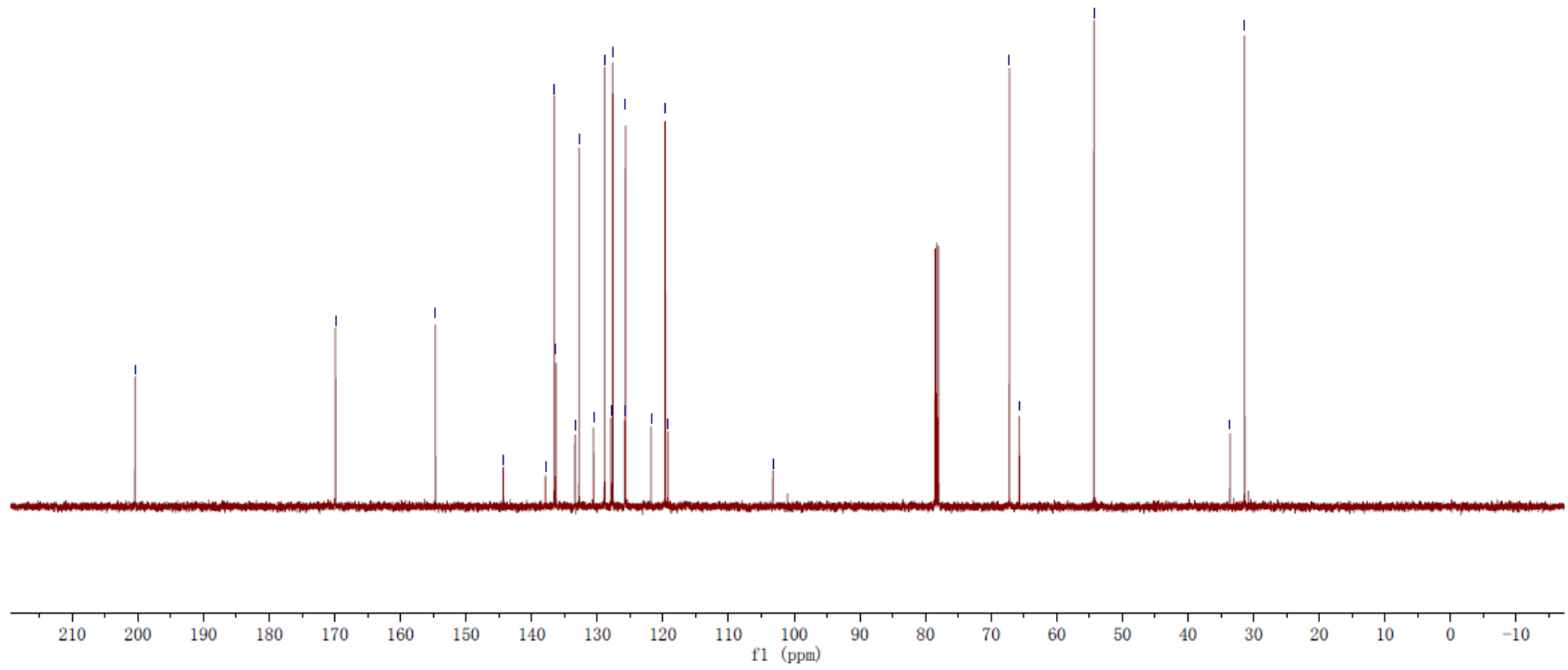

S59 

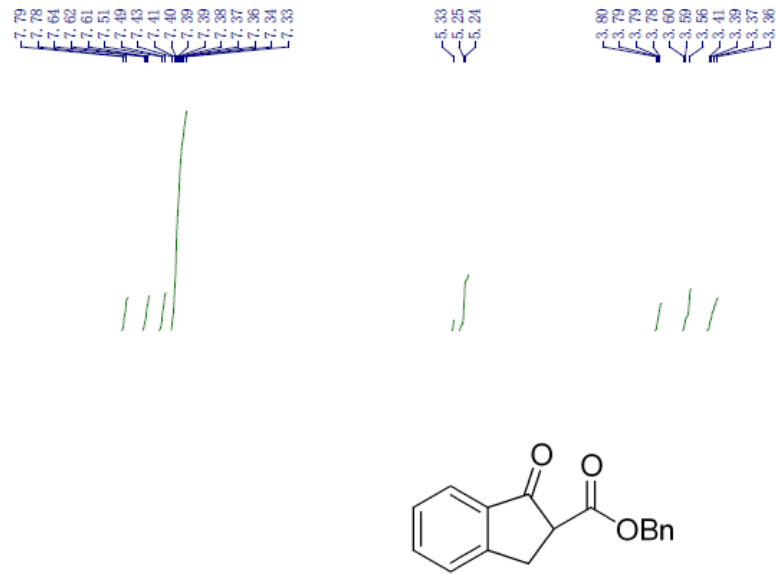

2s keto/enol=6:

$500 \mathrm{MHz} \mathrm{CDCl}_{3}$

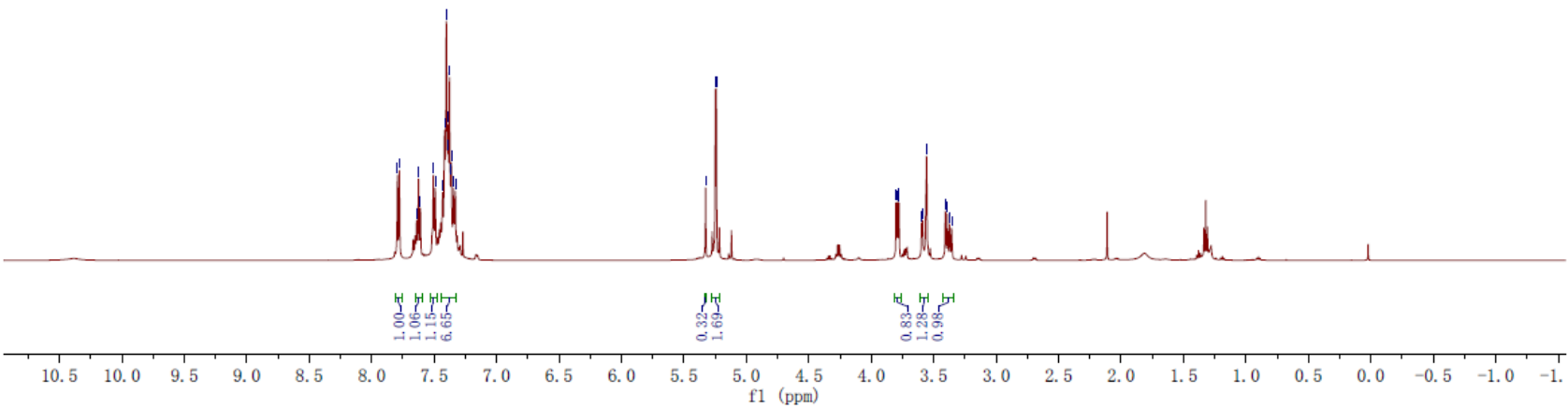

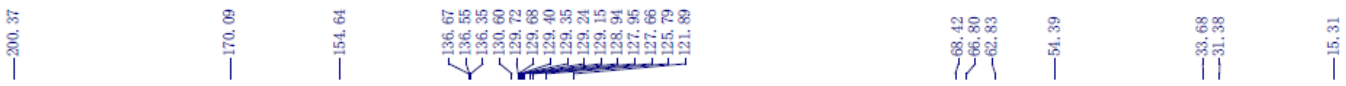

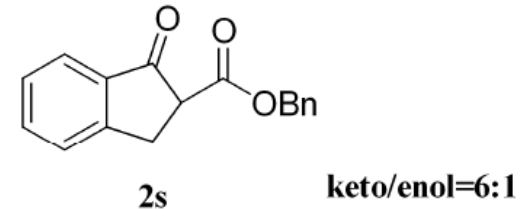

$126 \mathrm{MHz}, \mathrm{CDCl}_{3}$

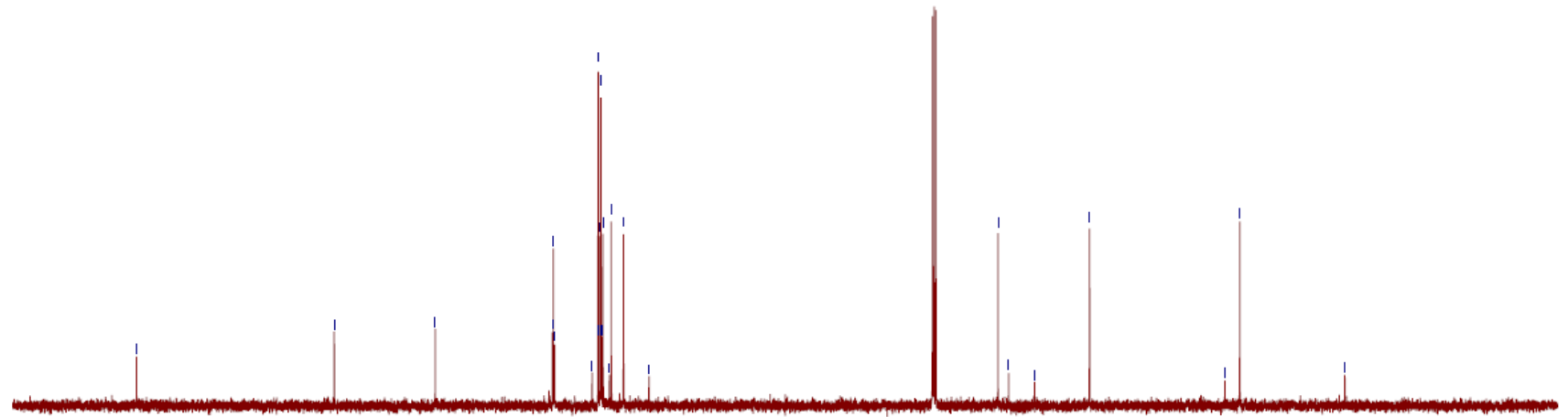

$210 \quad 200,190,2180$

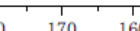

150

130

$110 \quad 100$

1
90
80 

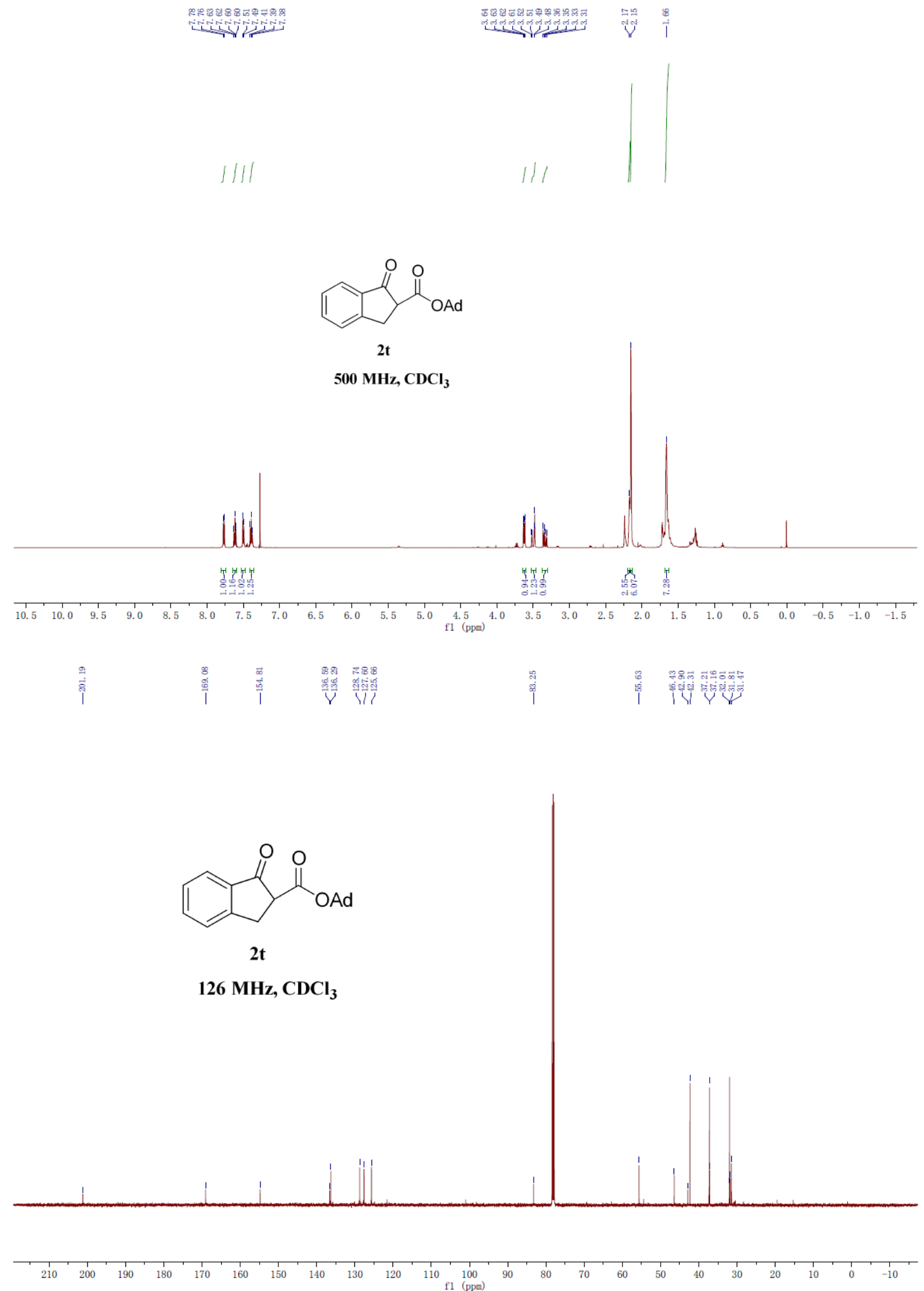


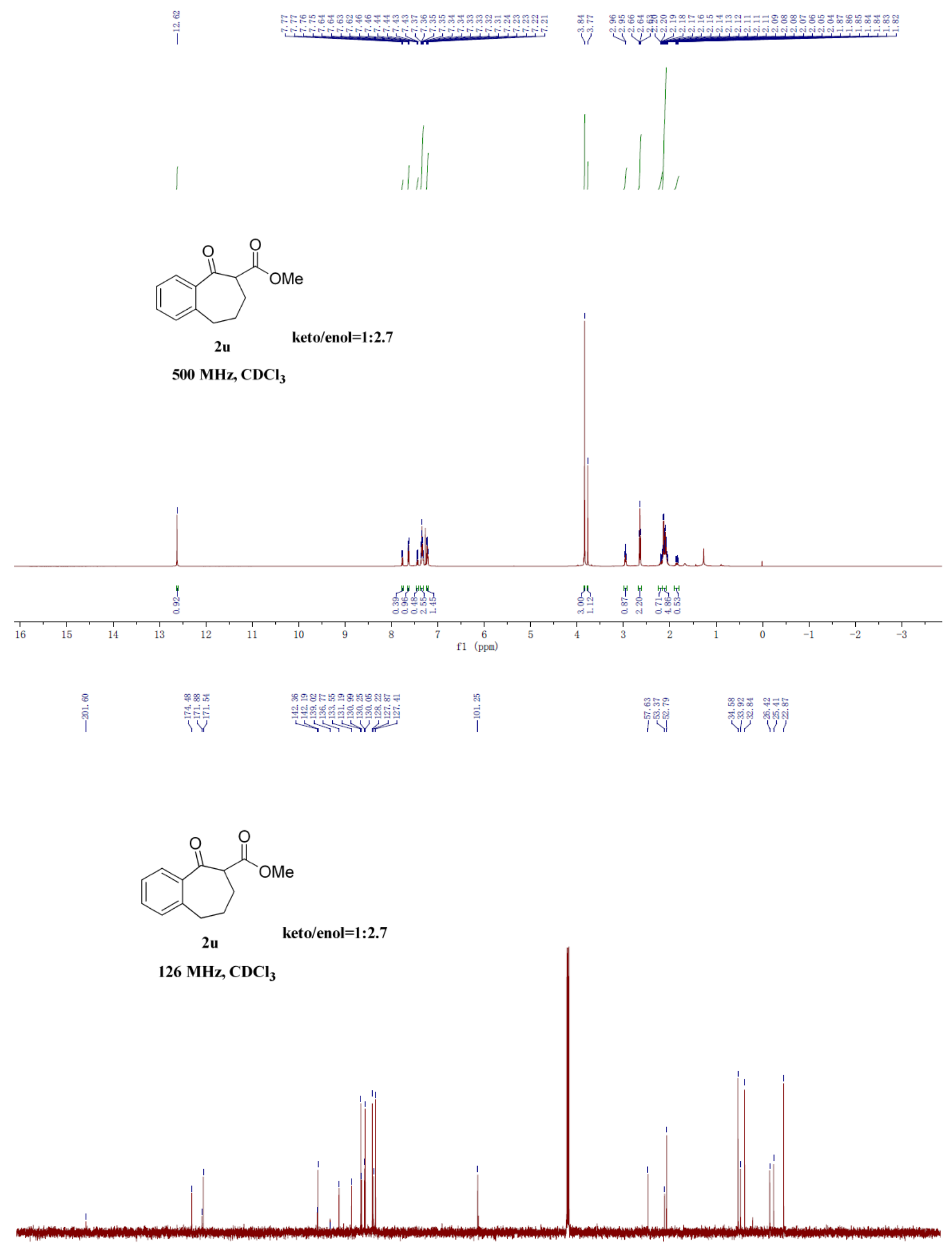

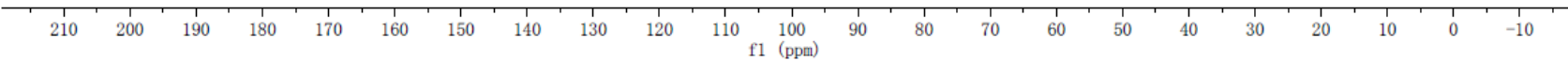


<smiles>CCOC(=O)C1COc2ccccc2C1=O</smiles>

$2 v$

keto/enol=2: 1

$500 \mathrm{MHz}, \mathrm{CDCl}_{3}$

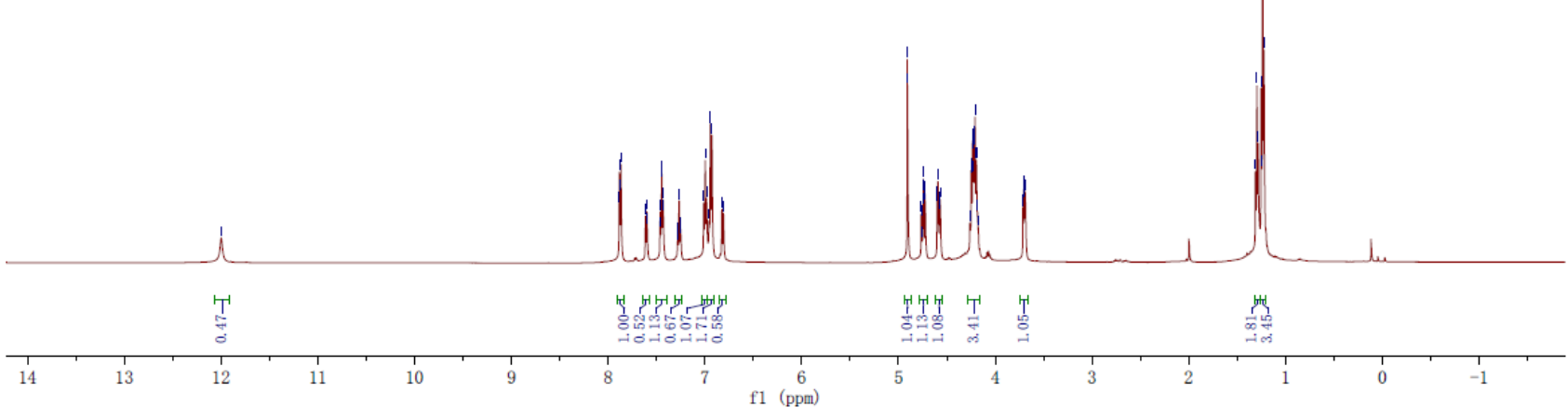<smiles>CCOC(=O)C1COc2ccccc2C1=O</smiles>

$126 \mathrm{MHz}, \mathrm{CDCl}_{3}$

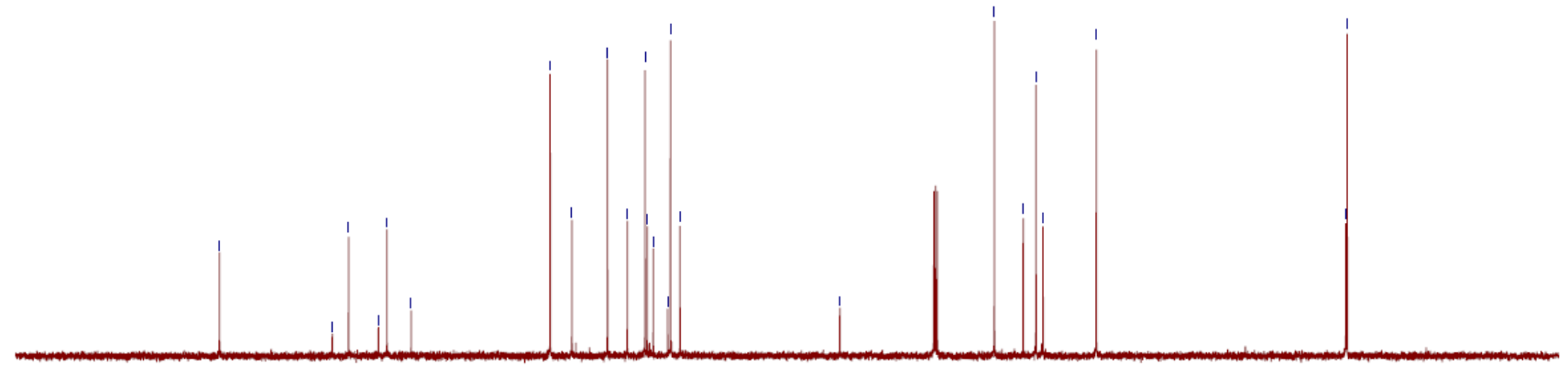

\section{$\begin{array}{llllllllllll}210 & 200 & 190 & 180 & 170 & 160 & 150 & 140 & 130 & 120 & 110 & 100 \\ \mathrm{f} 1 & (\mathrm{ppm})\end{array}$}



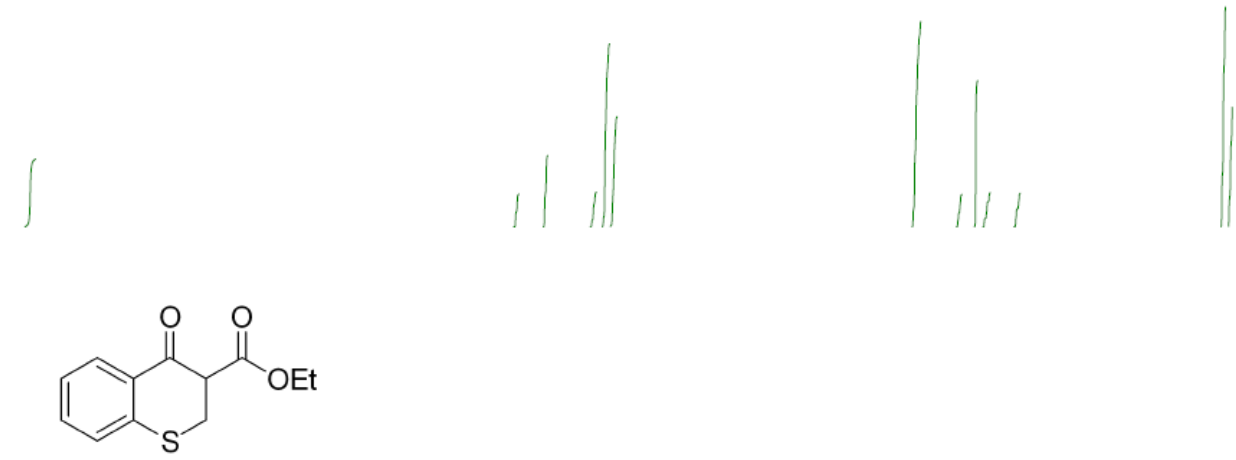

$2 w$

keto/enol=1:2

$500 \mathrm{MHz}, \mathrm{CDCl}_{3}$

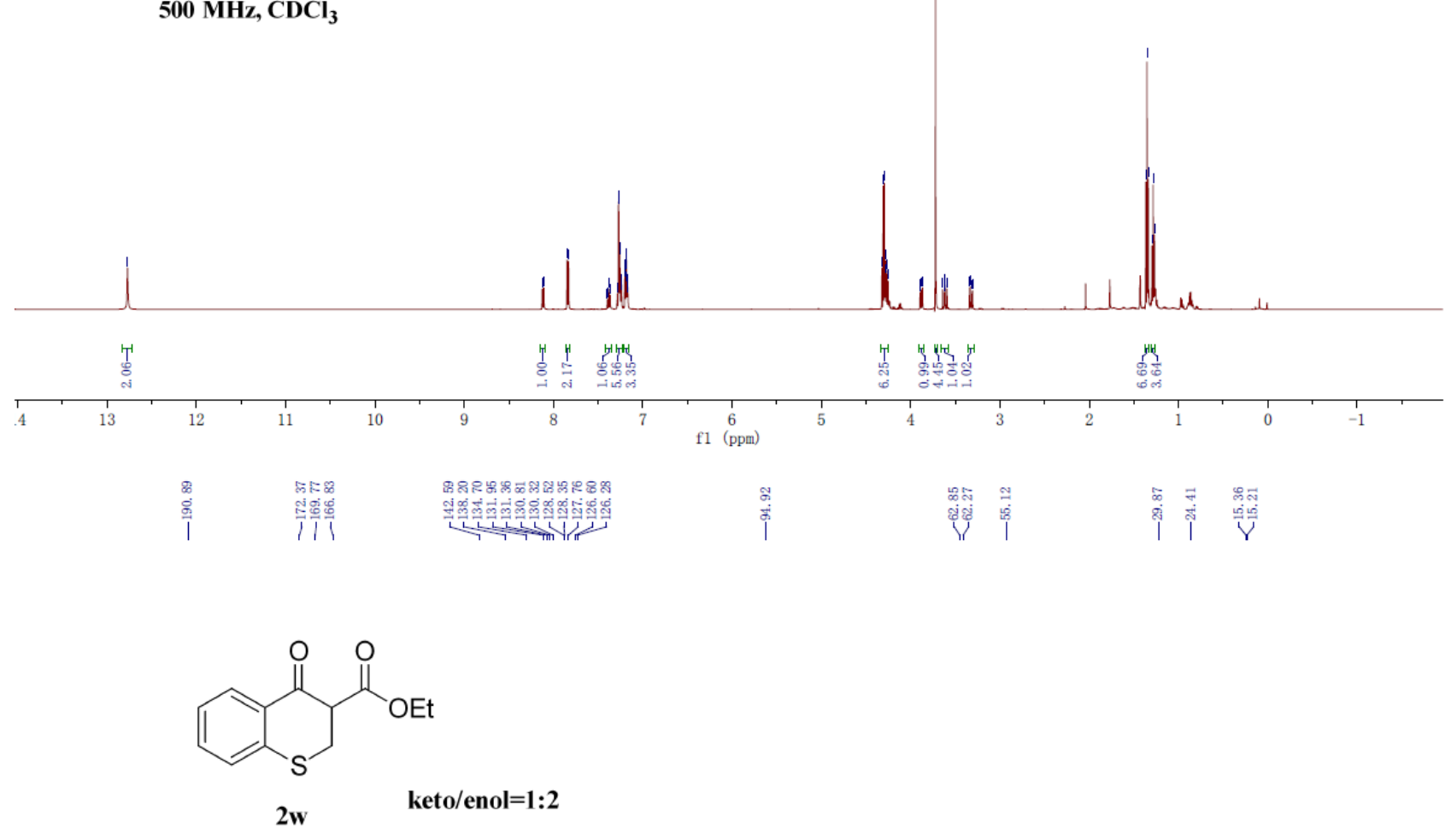

$126 \mathrm{MHz}^{\mathrm{CDCl}_{3}}$
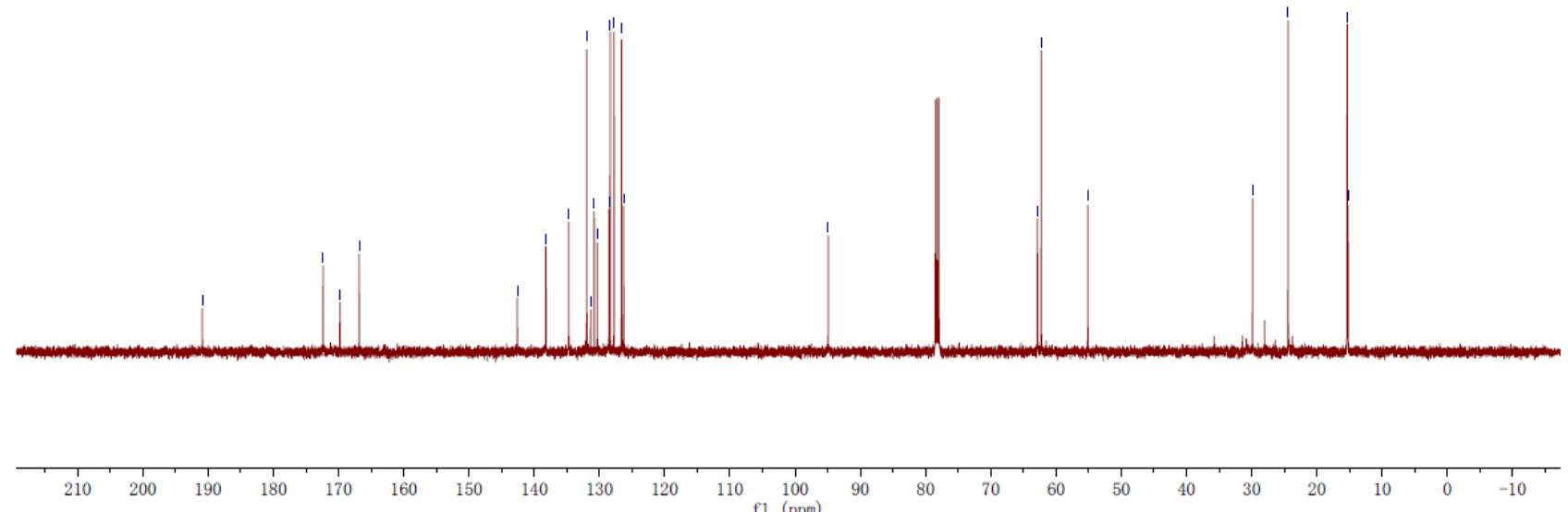


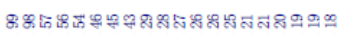

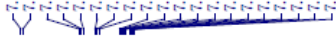

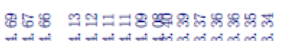

워오
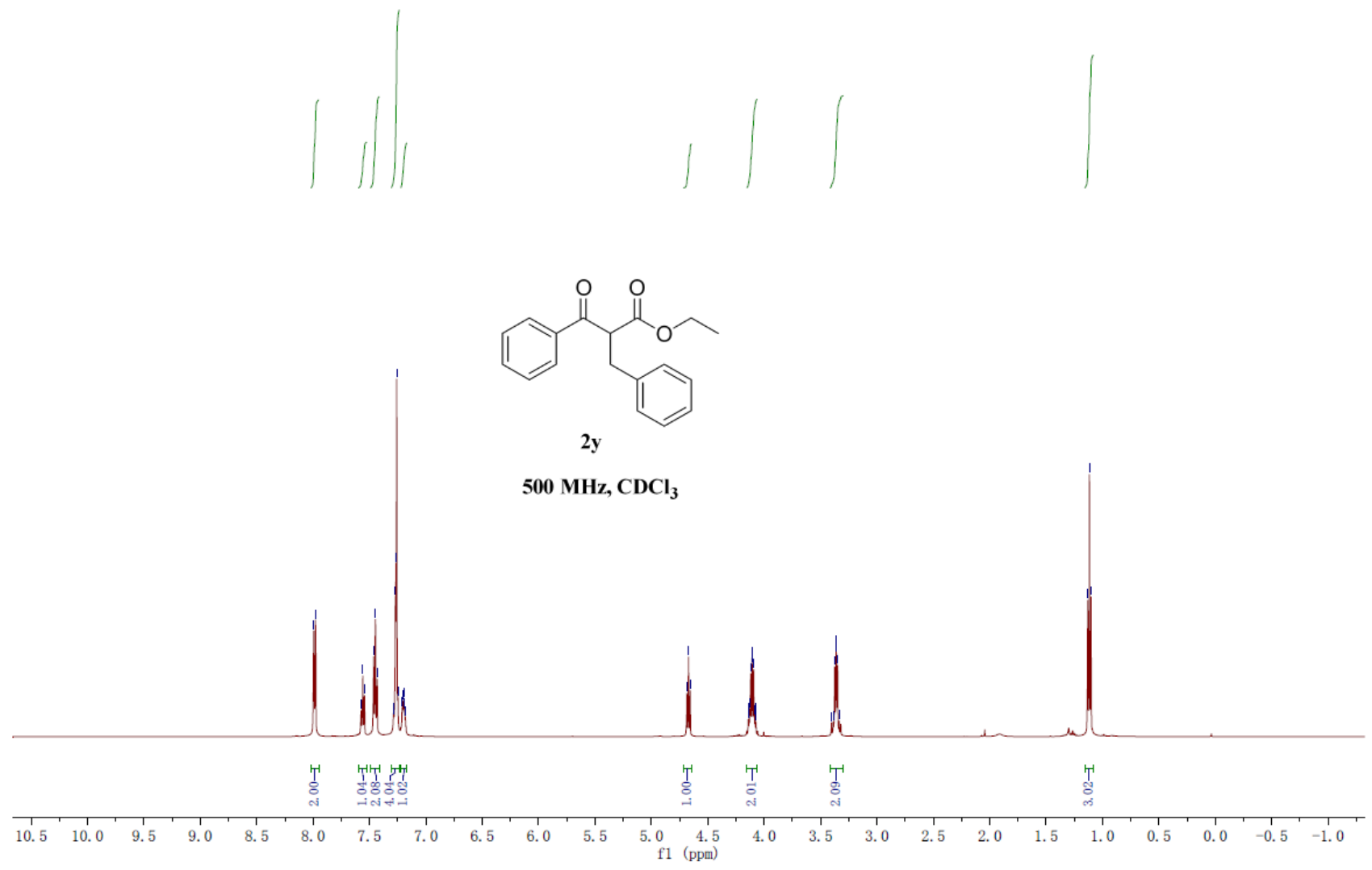

$2 y$

承

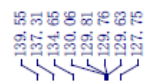

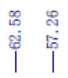

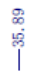

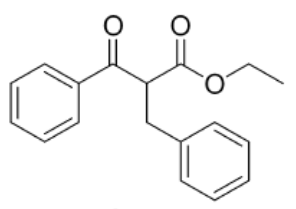

$2 \mathbf{y}$

$126 \mathrm{MHz}^{\mathrm{CDCl}_{3}}$

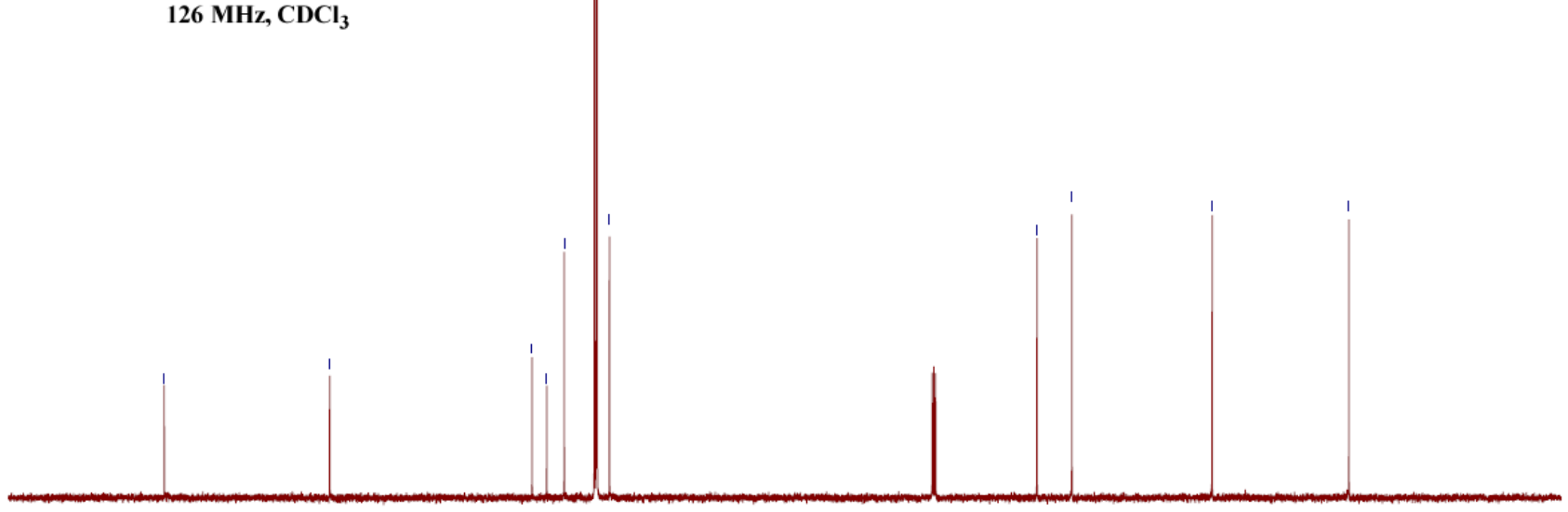

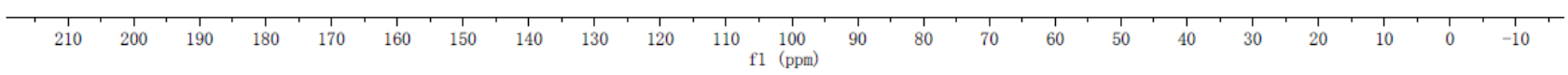

S65 


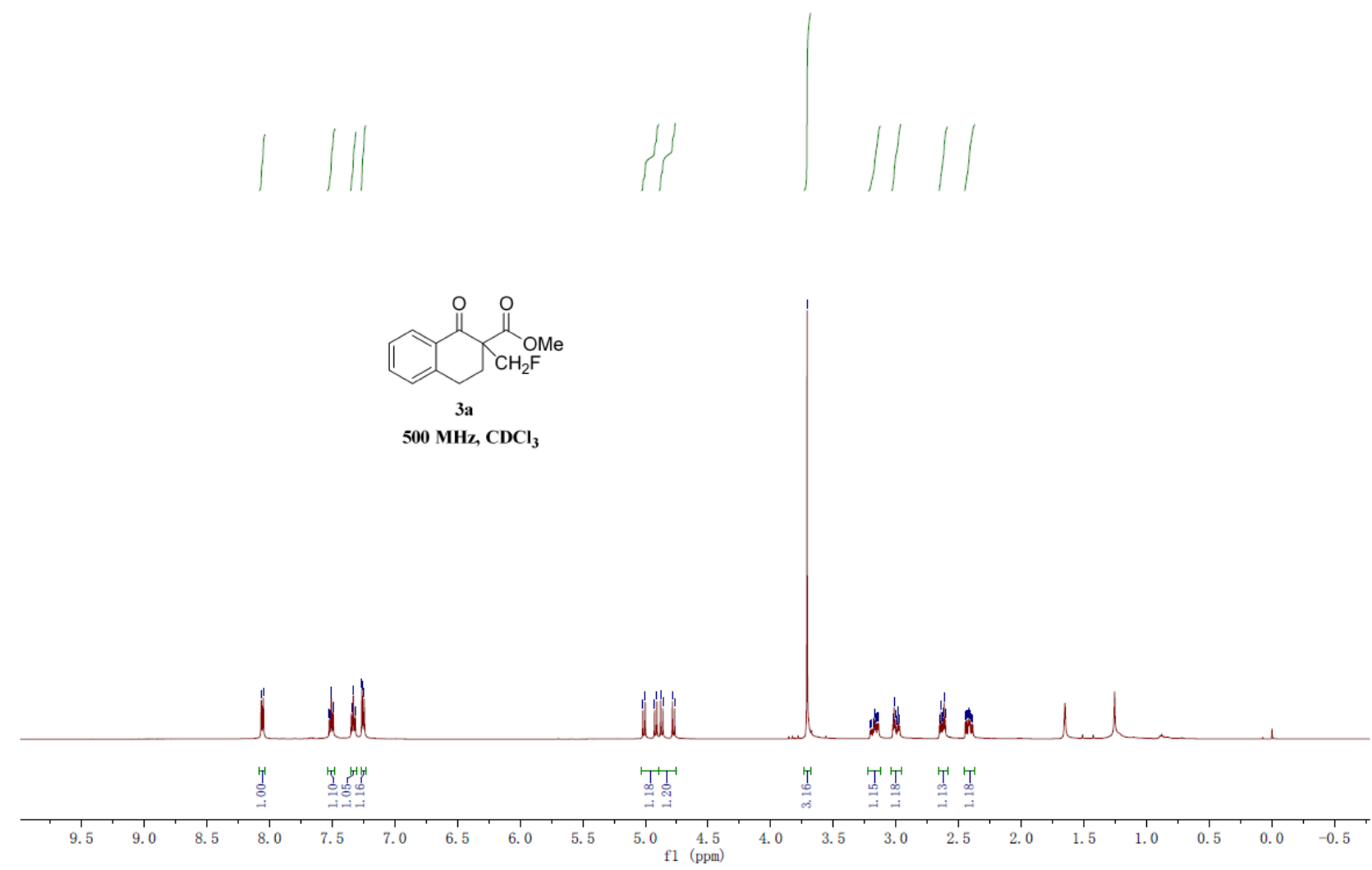

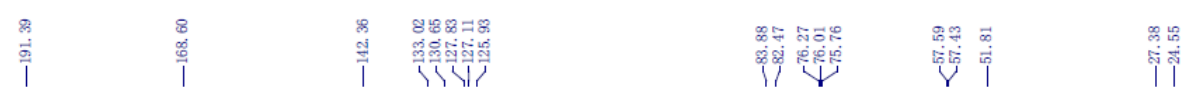
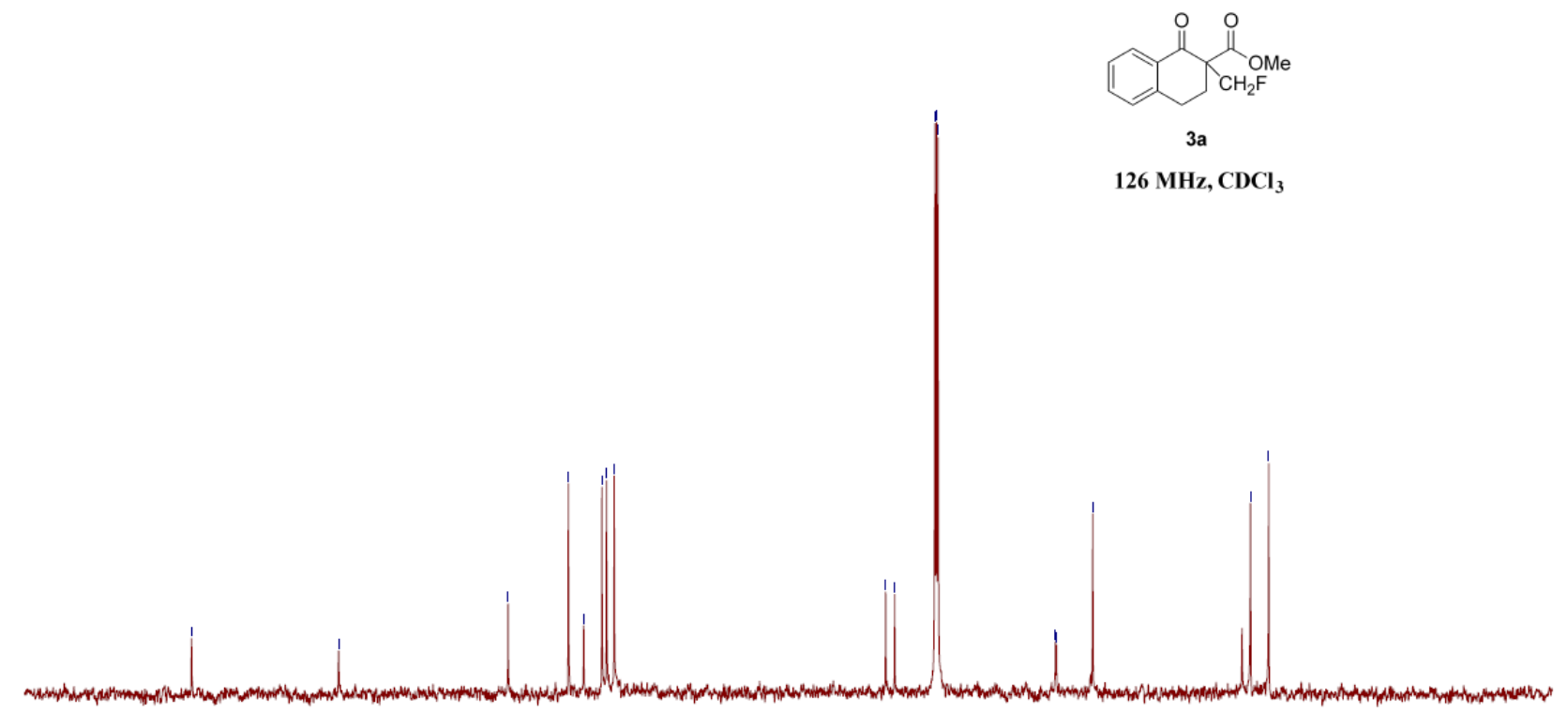

$126 \mathrm{MHz} \mathrm{CDCl}_{3}$

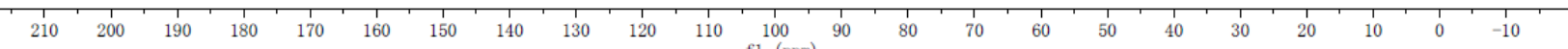




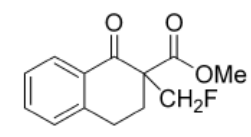

3a

$470 \mathrm{MHz}, \mathrm{CDCl}_{3}$
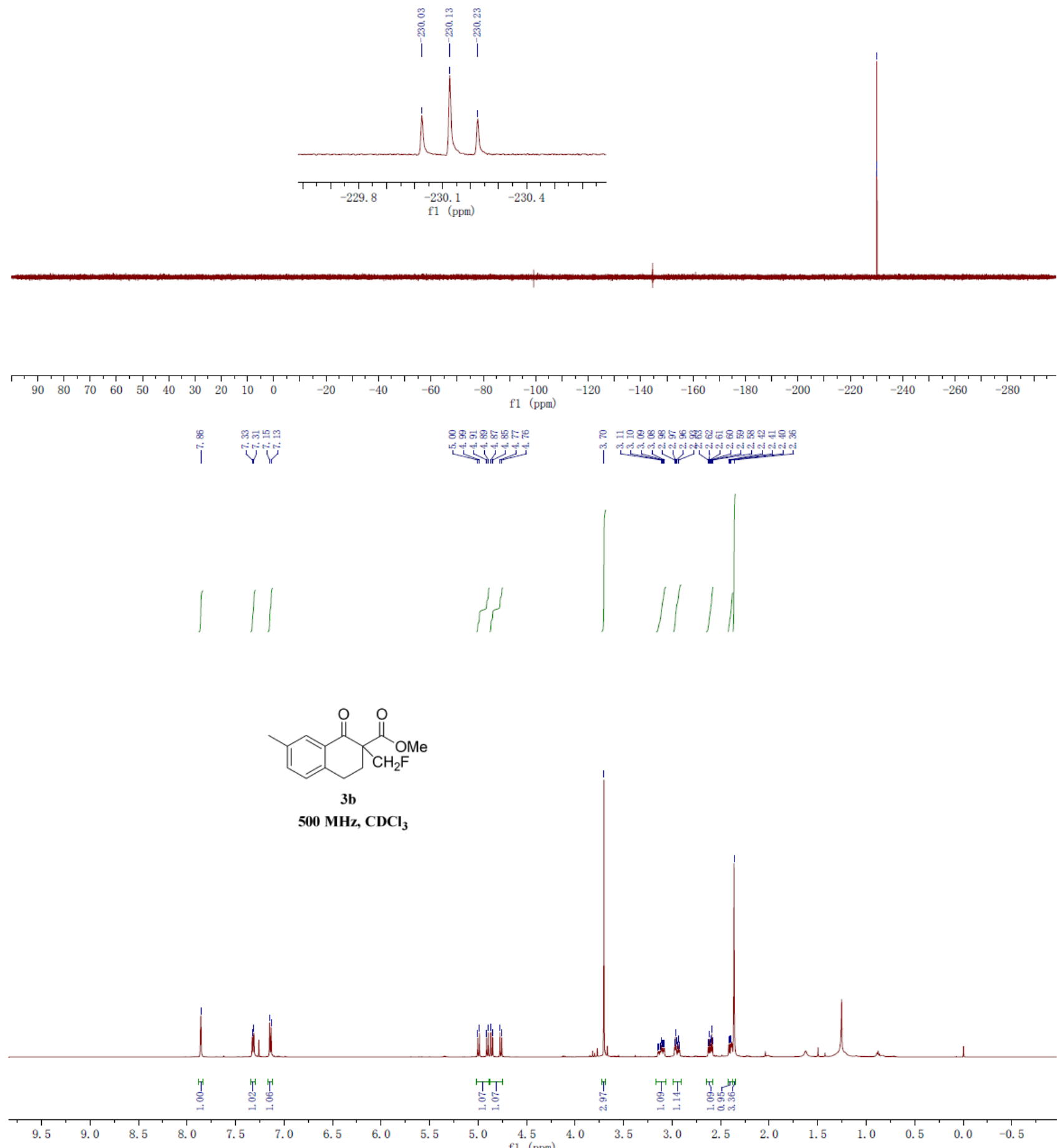

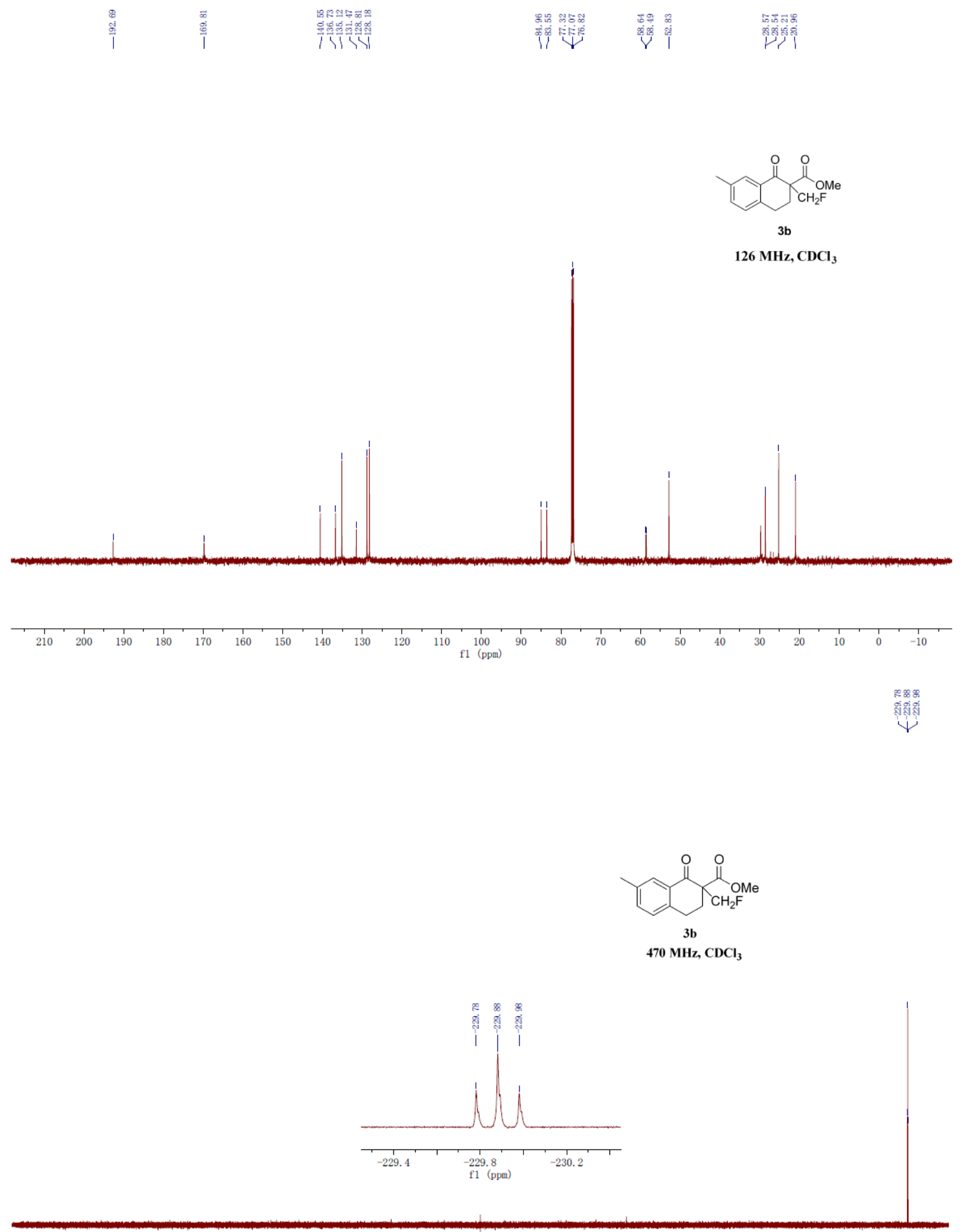

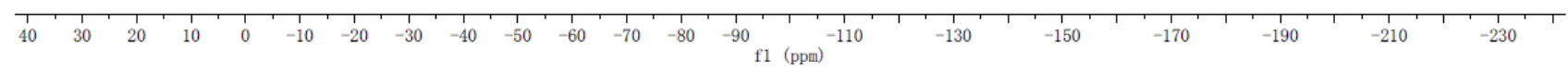



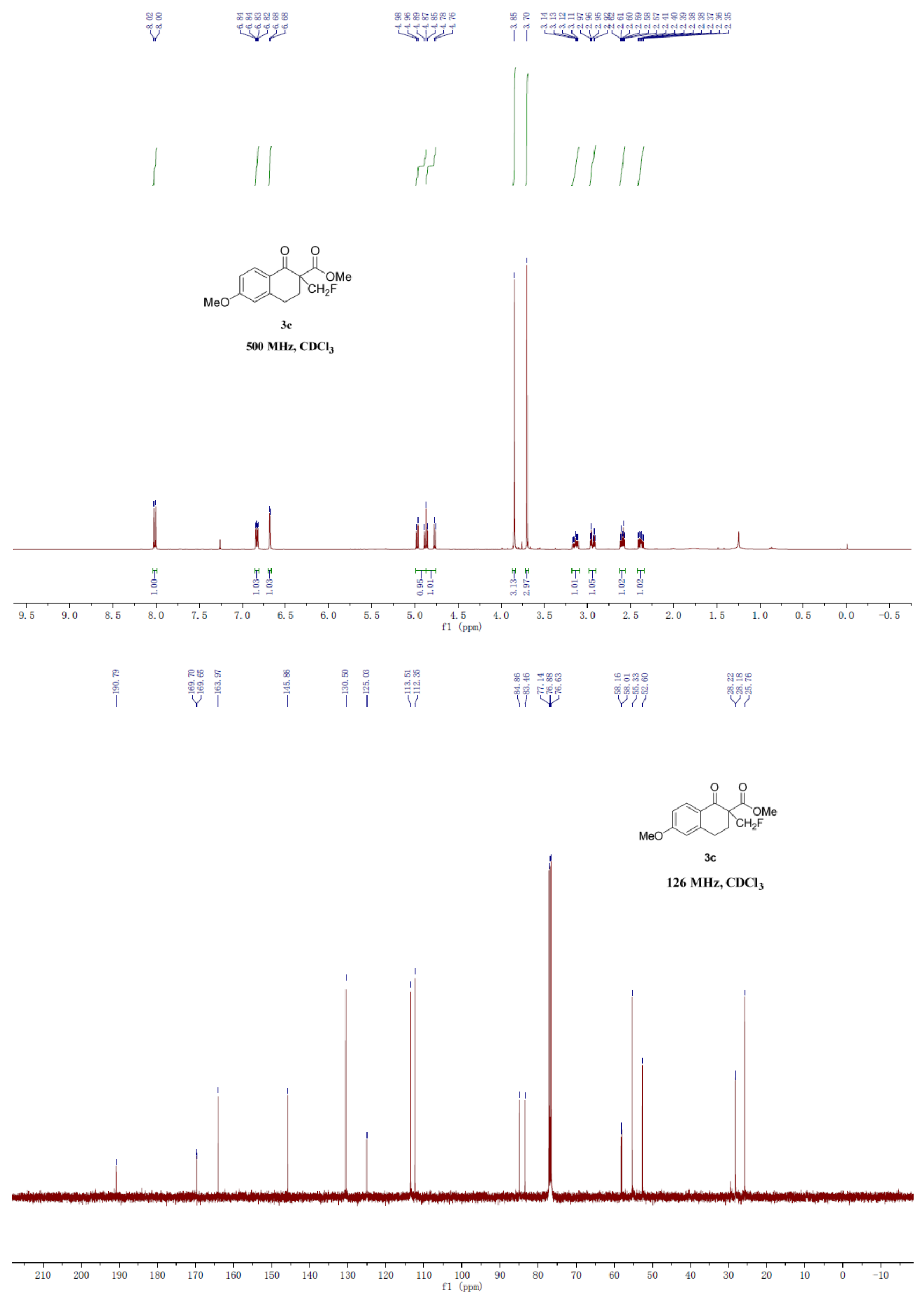


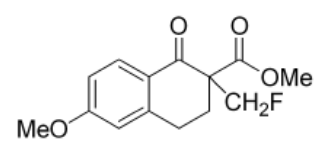

$3 \mathbf{c}$

$470 \mathrm{MHz}, \mathrm{CDCl}_{3}$

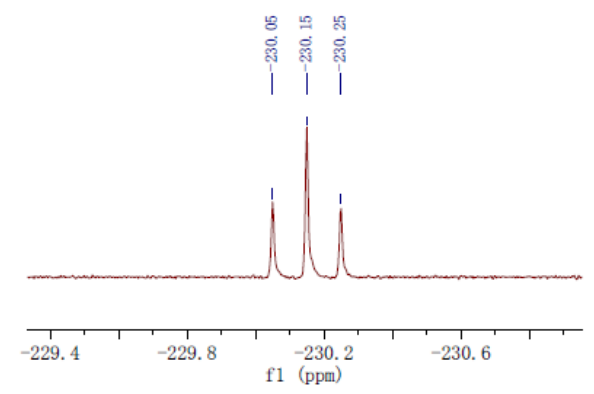

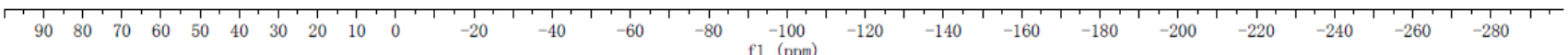

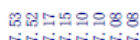

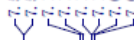
888585R日
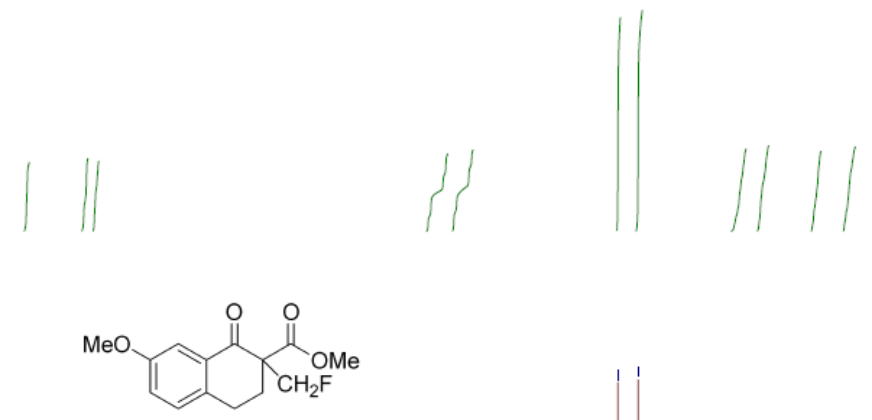

$3 d$

$500 \mathrm{MHz} \mathrm{CDCl}_{3}$

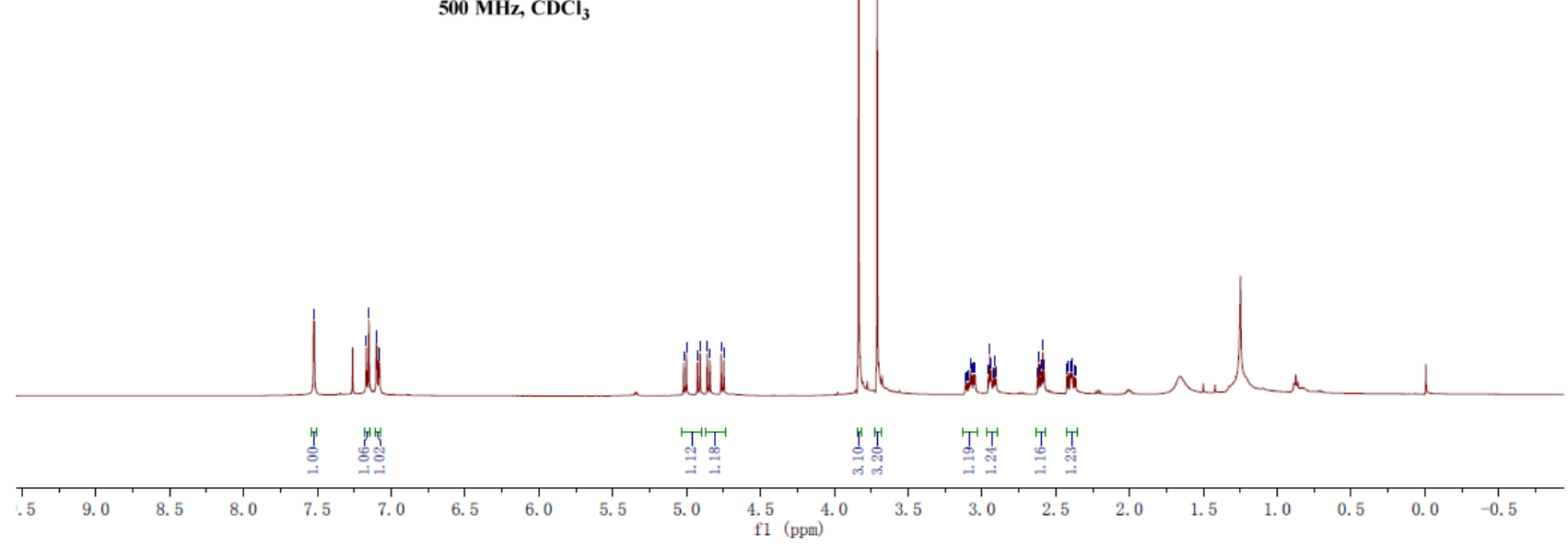



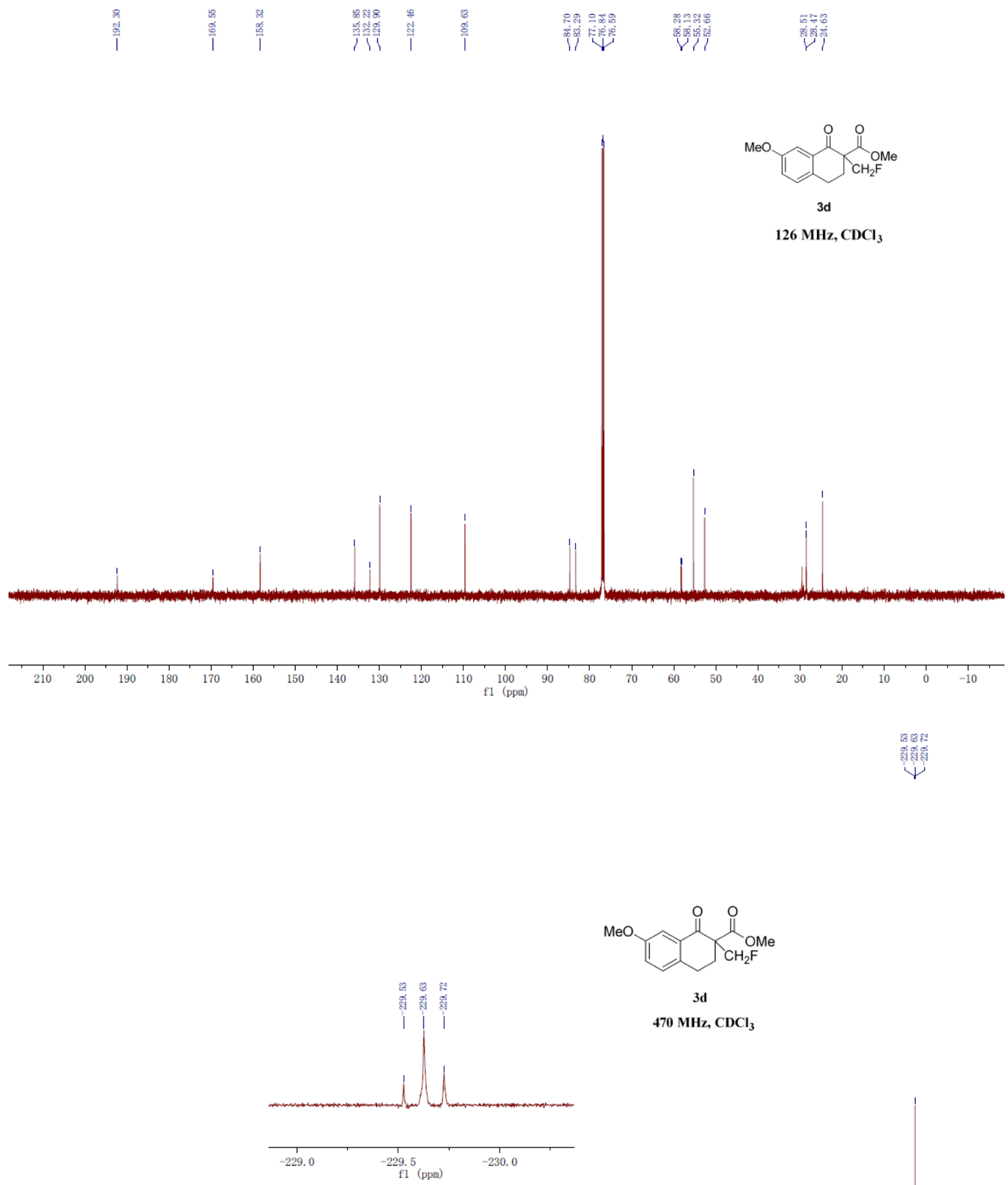

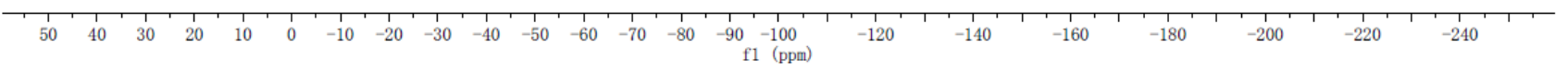




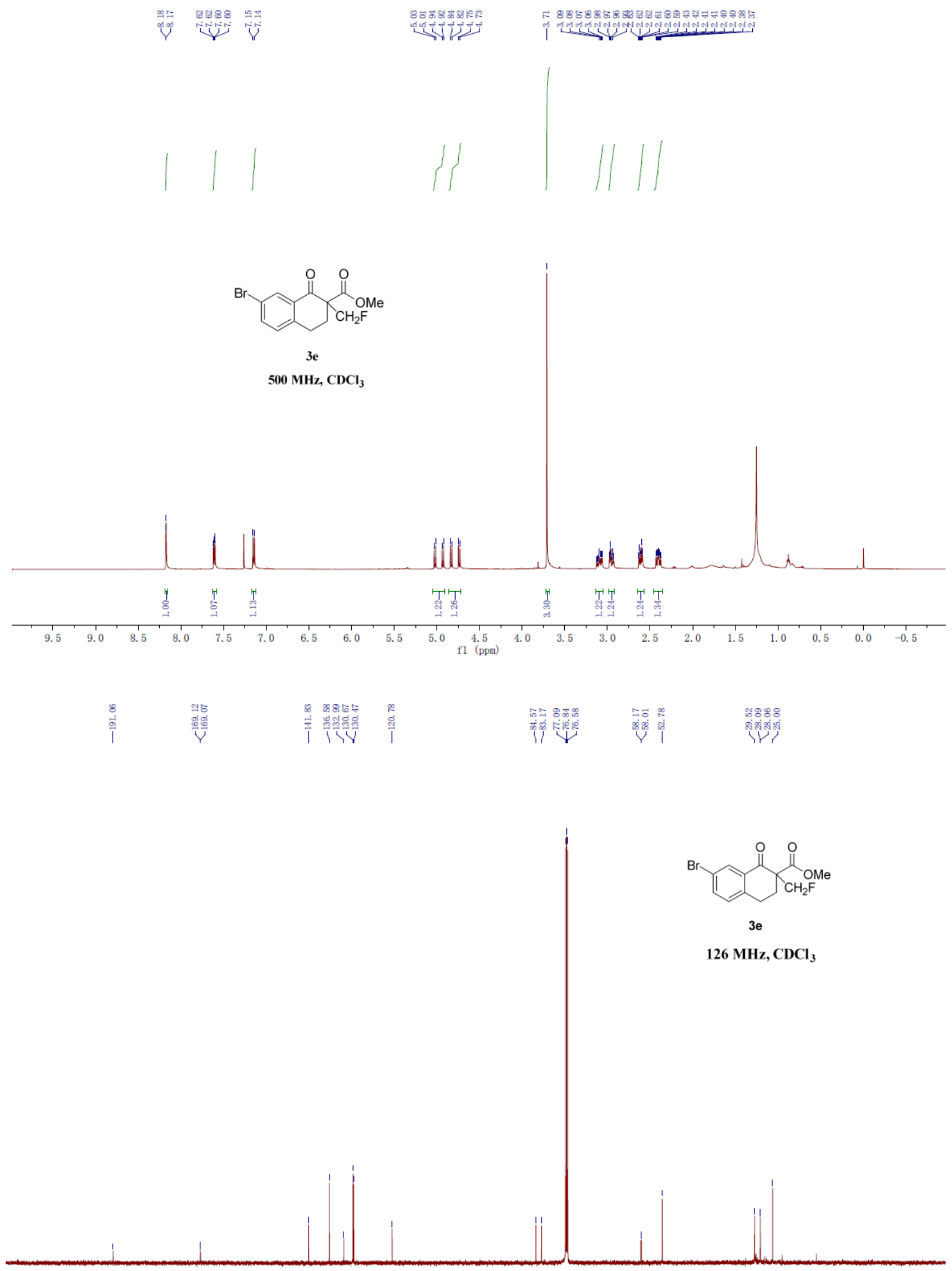

9.5
9.0
9.5

$500 \mathrm{MHz}, \mathrm{CDCl}_{3}$ 

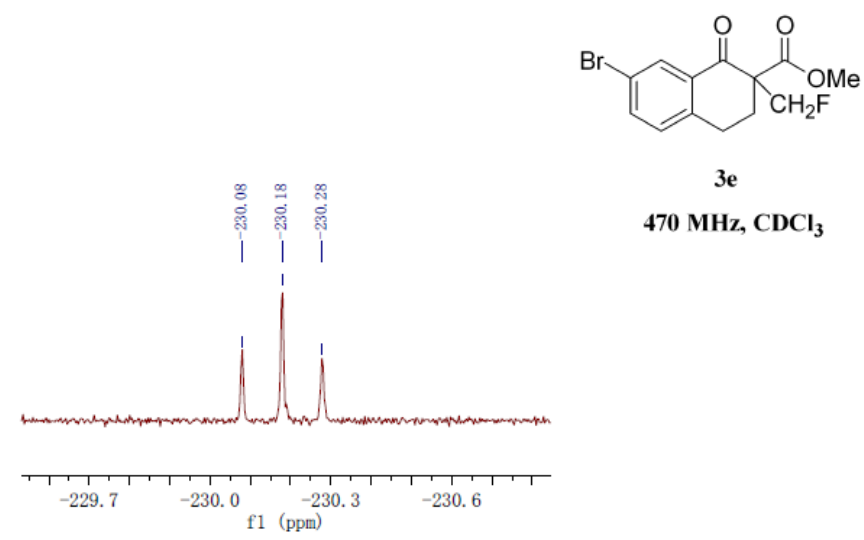

3 e

$470 \mathrm{MHz}, \mathrm{CDCl}_{3}$
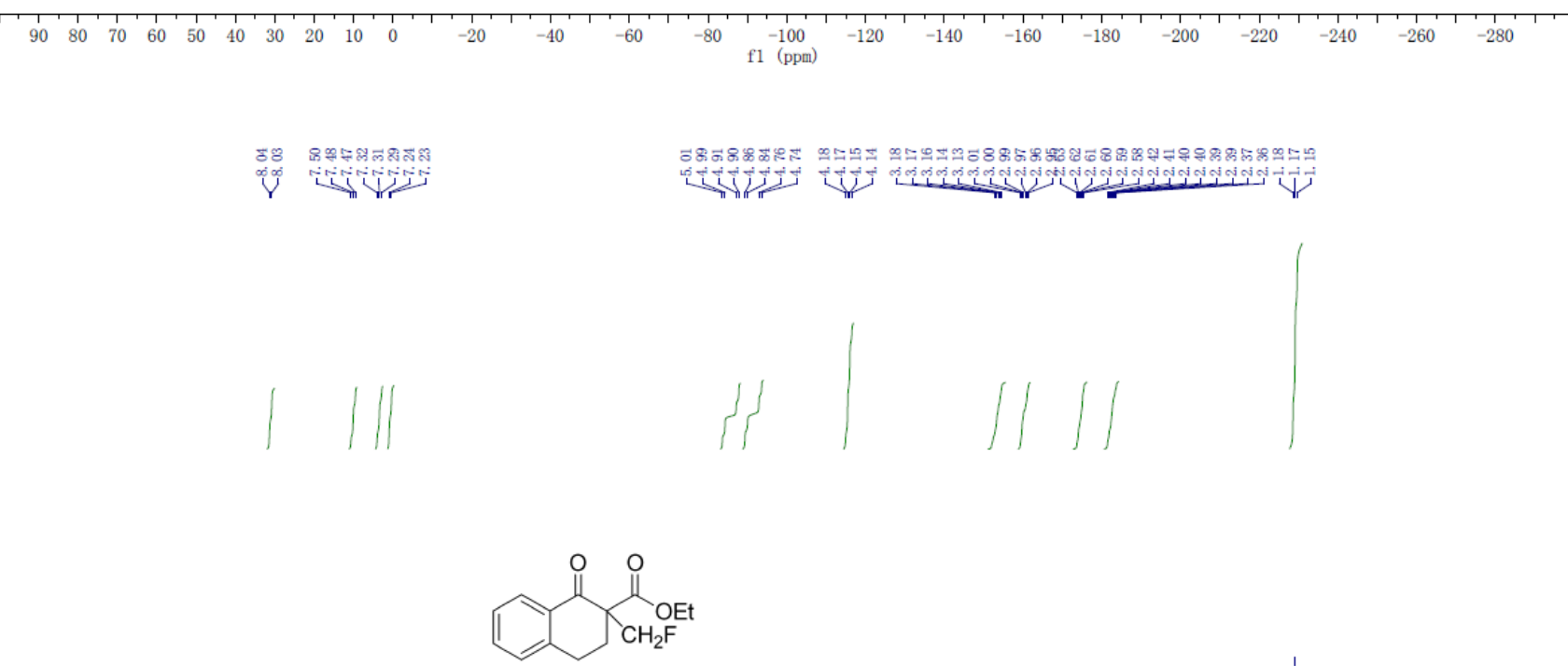

$3 f$

$500 \mathrm{MHz}, \mathrm{CDCl}_{3}$

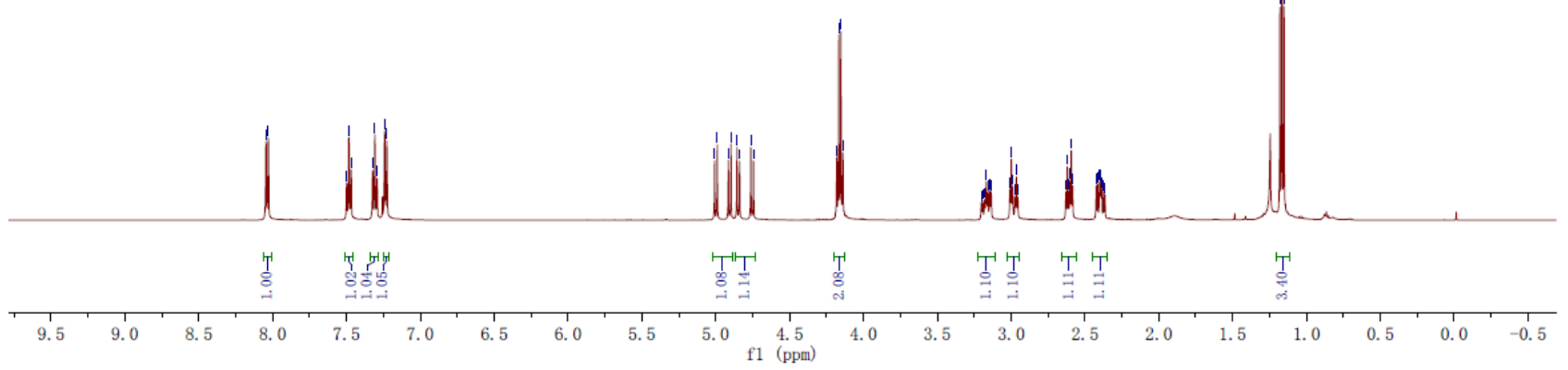




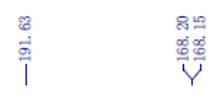

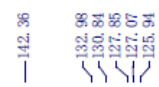

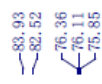

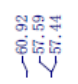

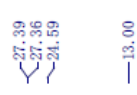

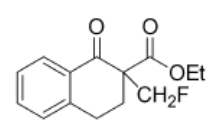

$3 f$
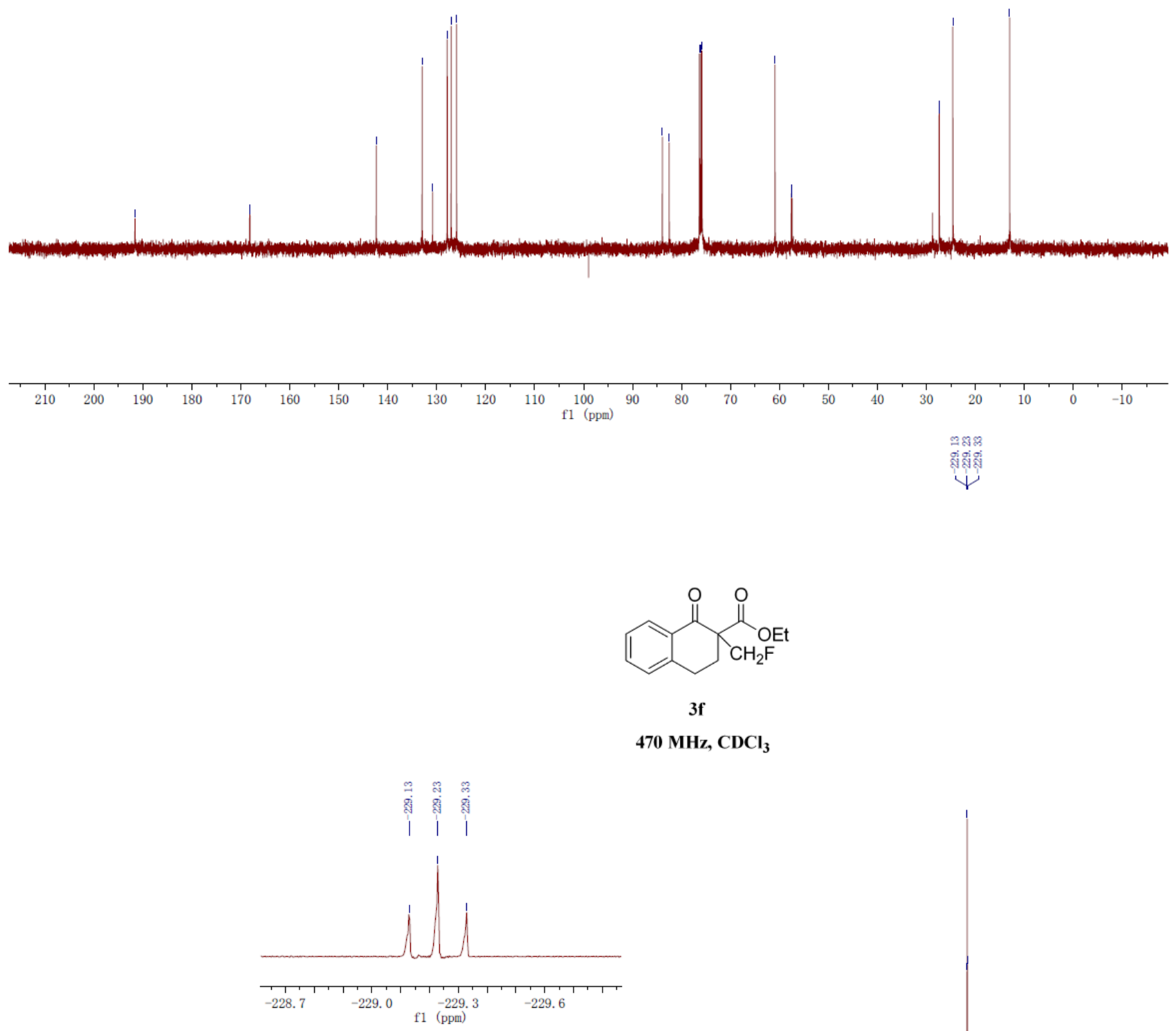

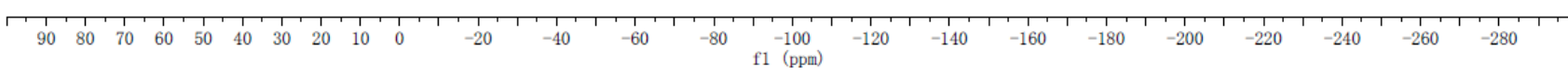

S74 

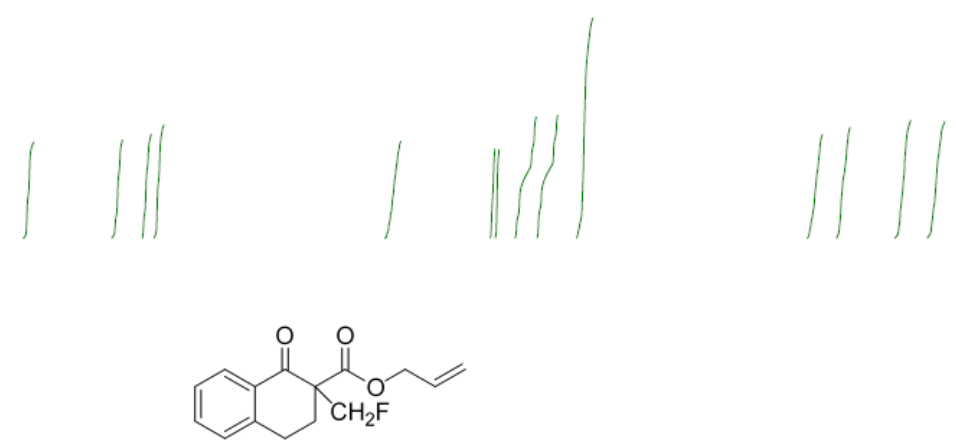

$3 g$

$500 \mathrm{MHz}, \mathrm{CDCl}_{3}$

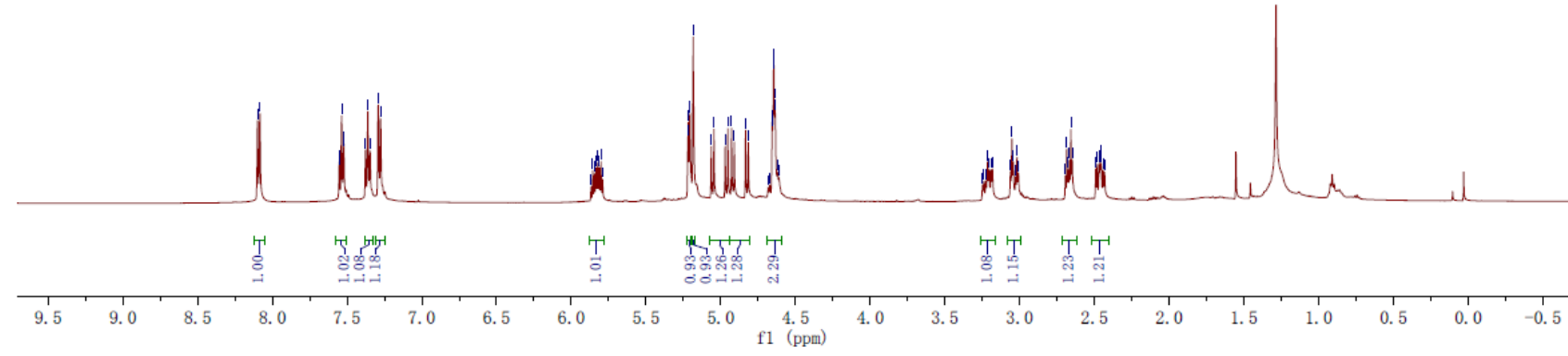

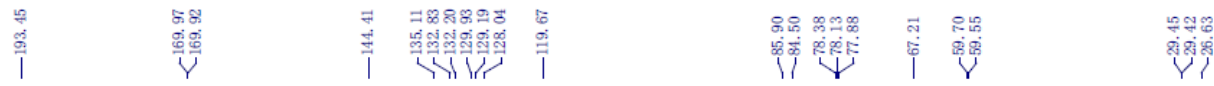

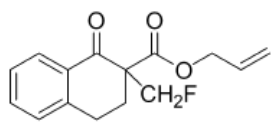

$3 g$

$126 \mathrm{MHz}^{\mathrm{CDCl}_{3}}$

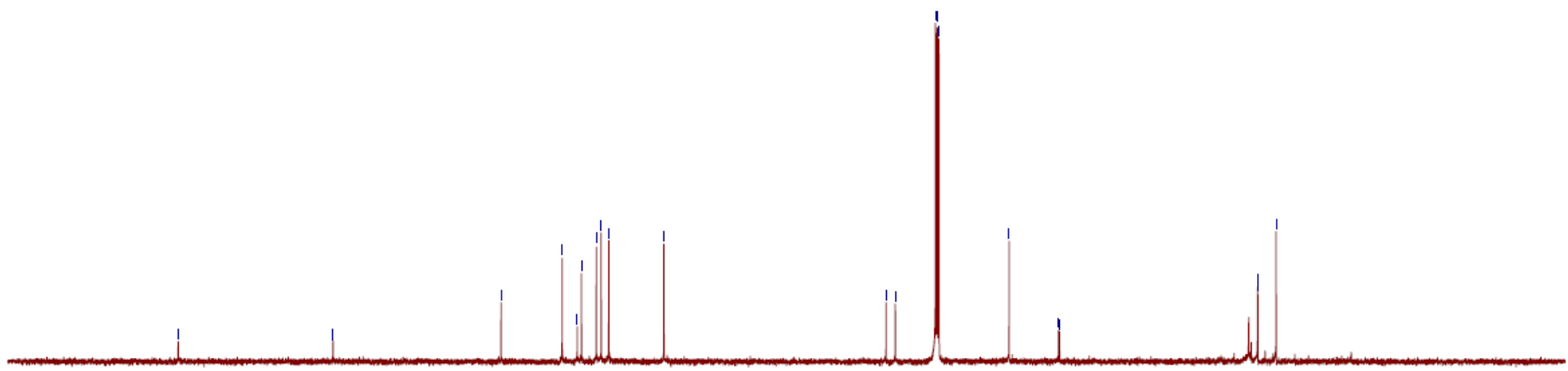

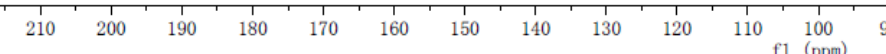




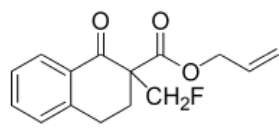

$3 \mathrm{~g}$

$470 \mathrm{MHz}, \mathrm{CDCl}_{3}$

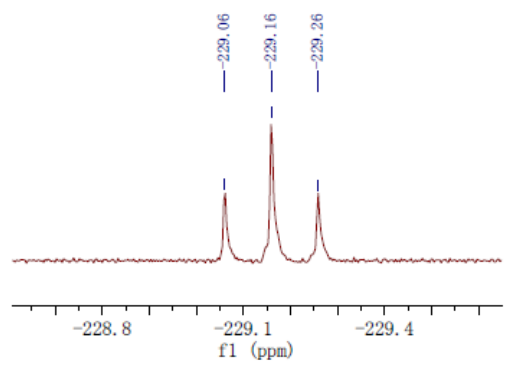

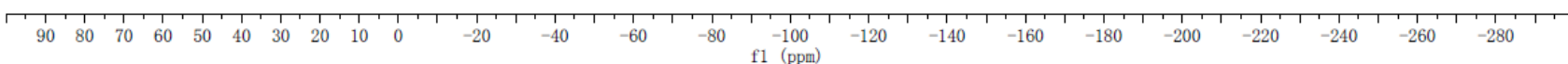

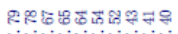

rivitivitis

în

\section{1}
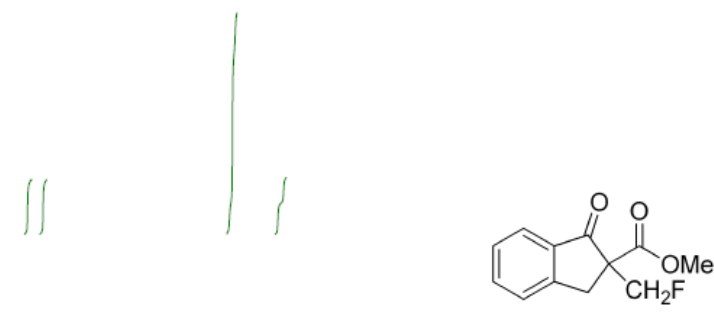

$3 \mathrm{~h}$

$500 \mathrm{MHz}, \mathrm{CDCl}_{3}$

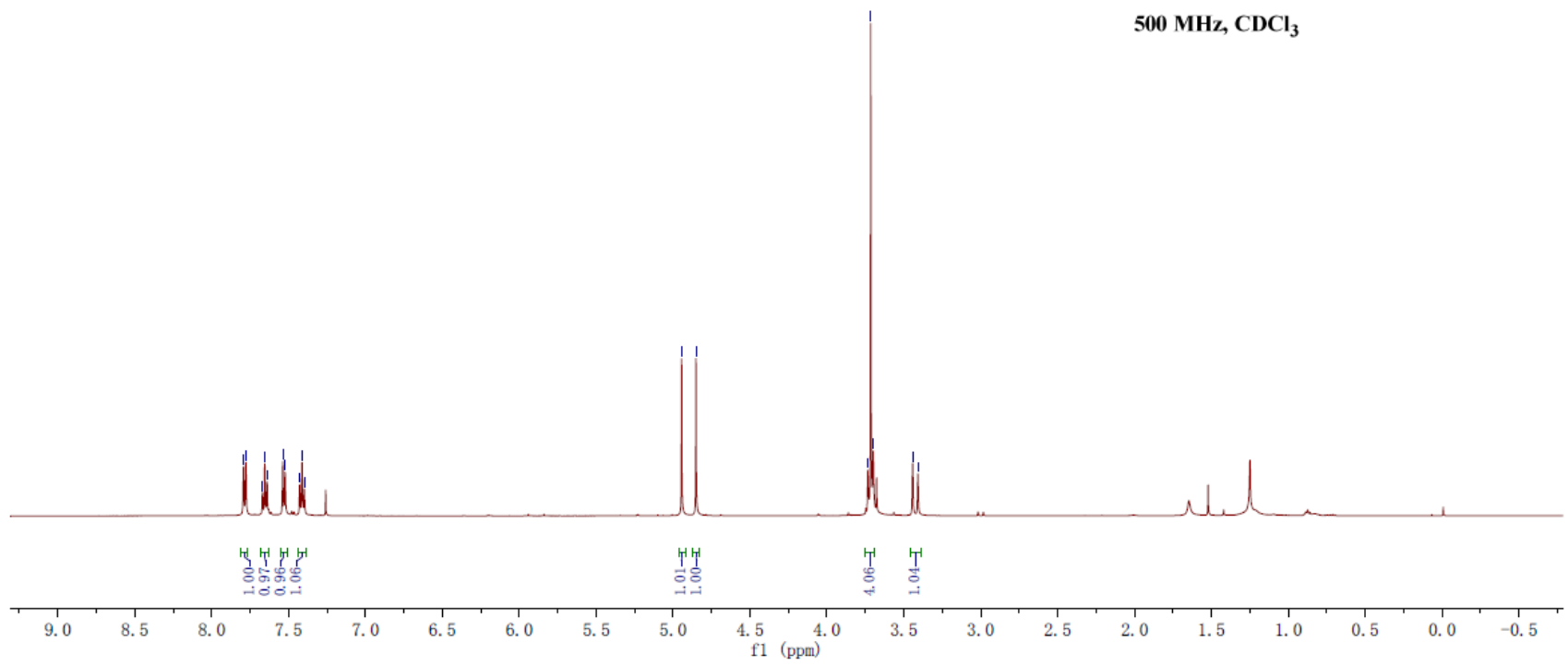




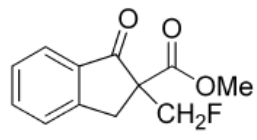

3h

$126 \mathrm{MHz}, \mathrm{CDCl}_{3}$
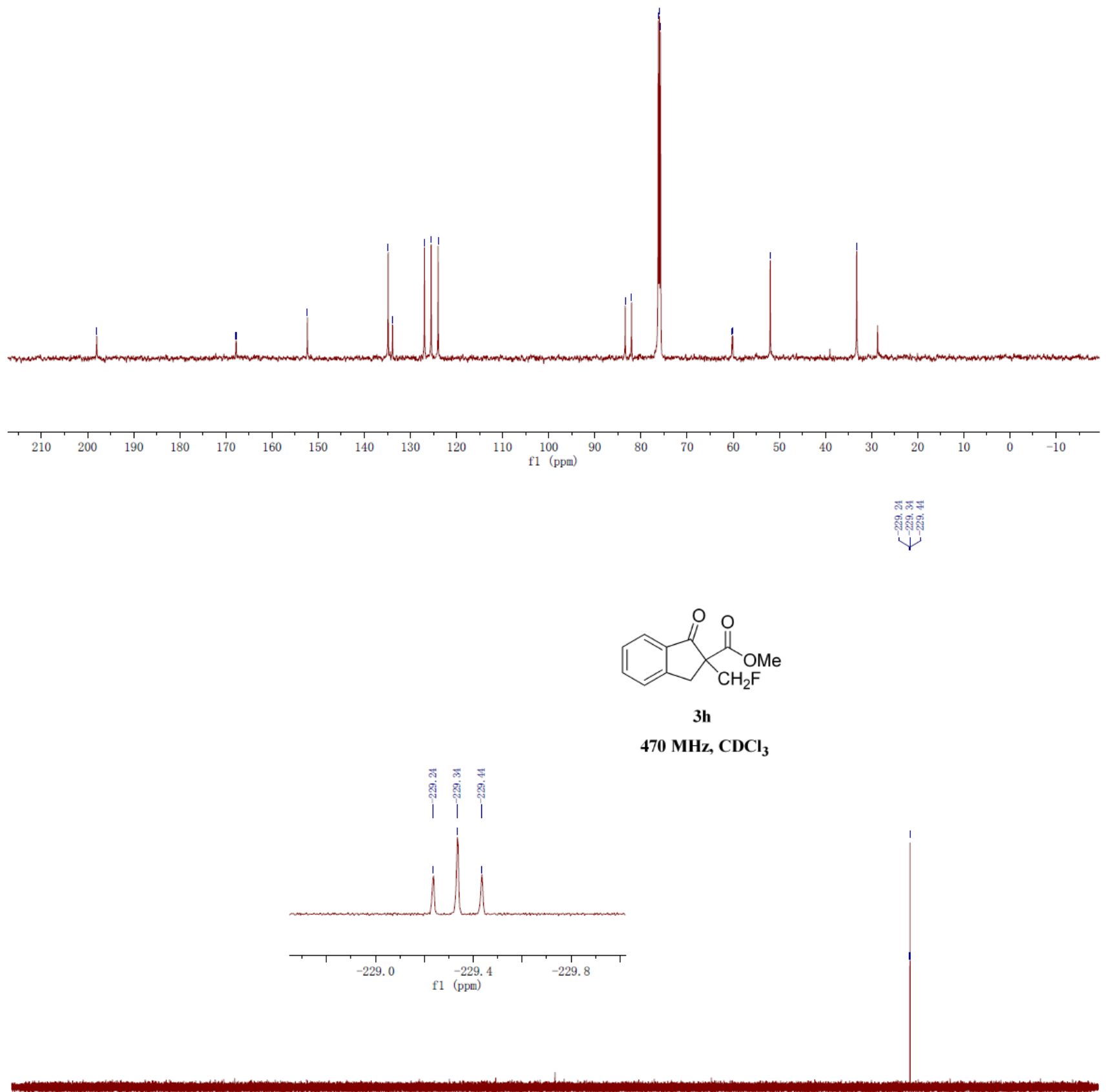

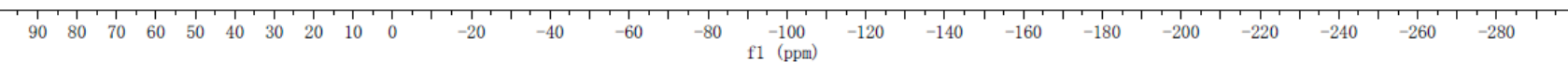




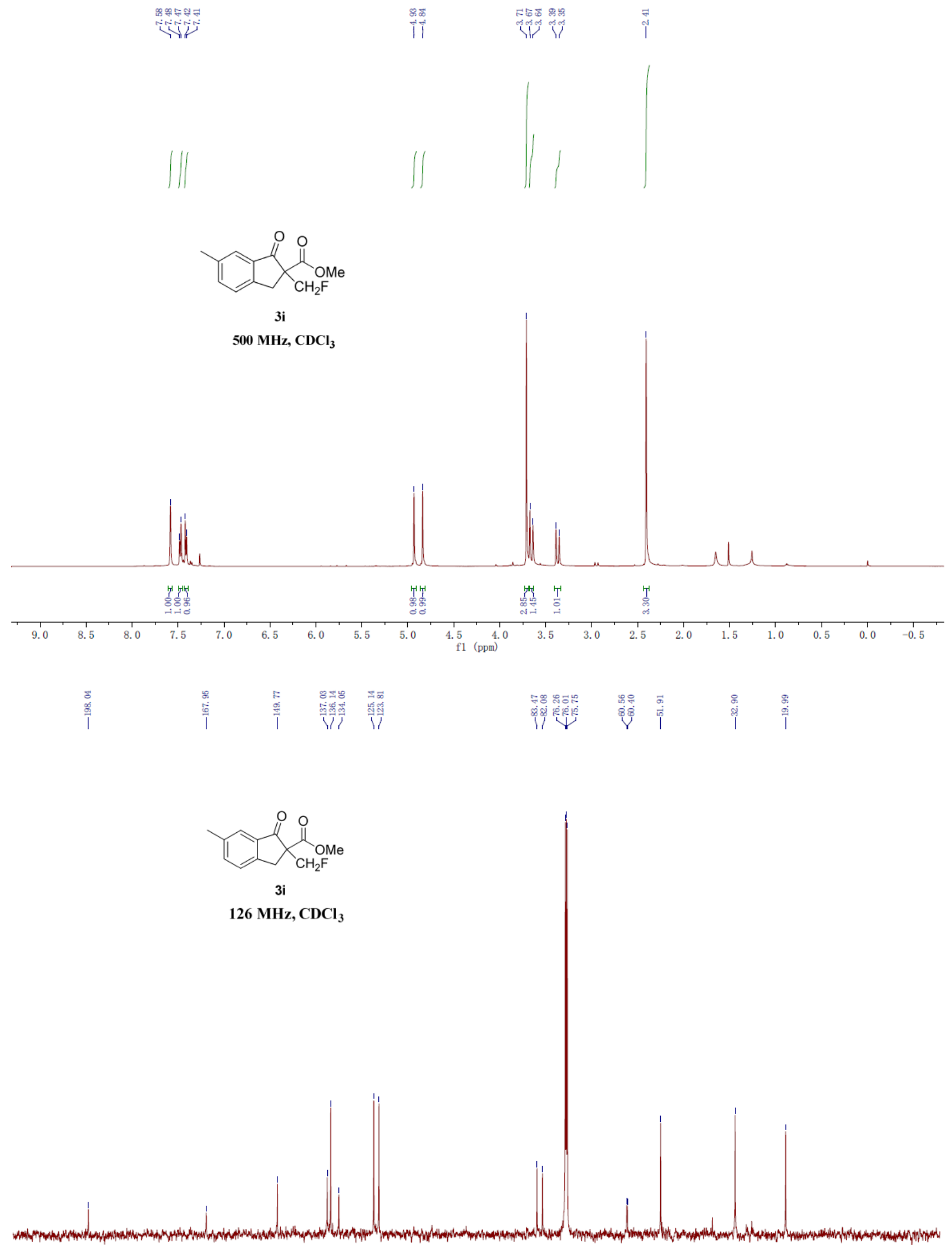

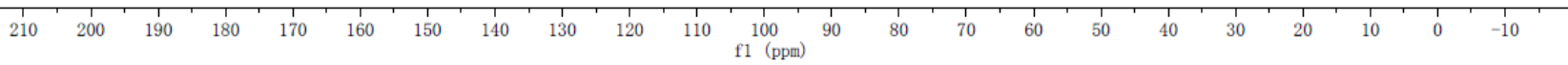




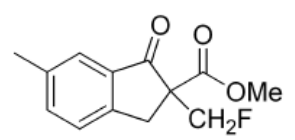

$3 i$

$470 \mathrm{MHz}, \mathrm{CDCl}_{3}$

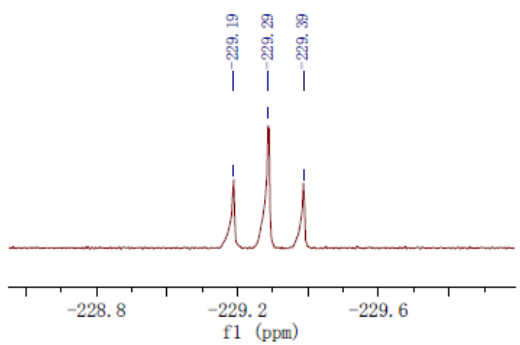

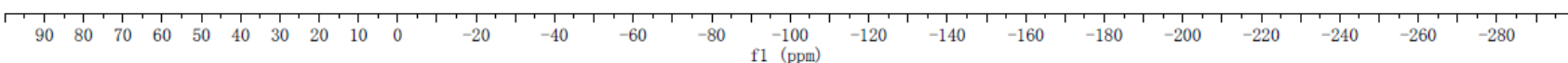

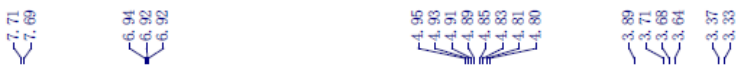
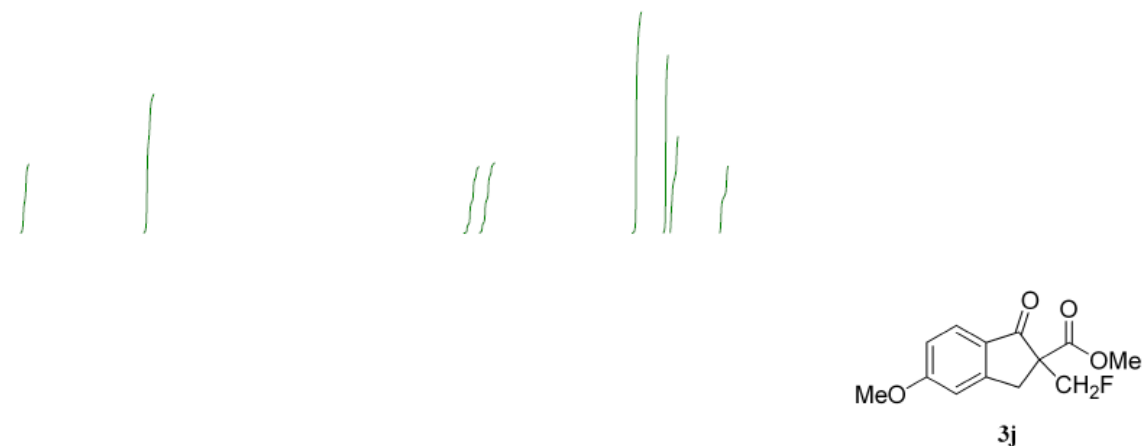

$500 \mathrm{MHz}, \mathrm{CDCl}_{3}$

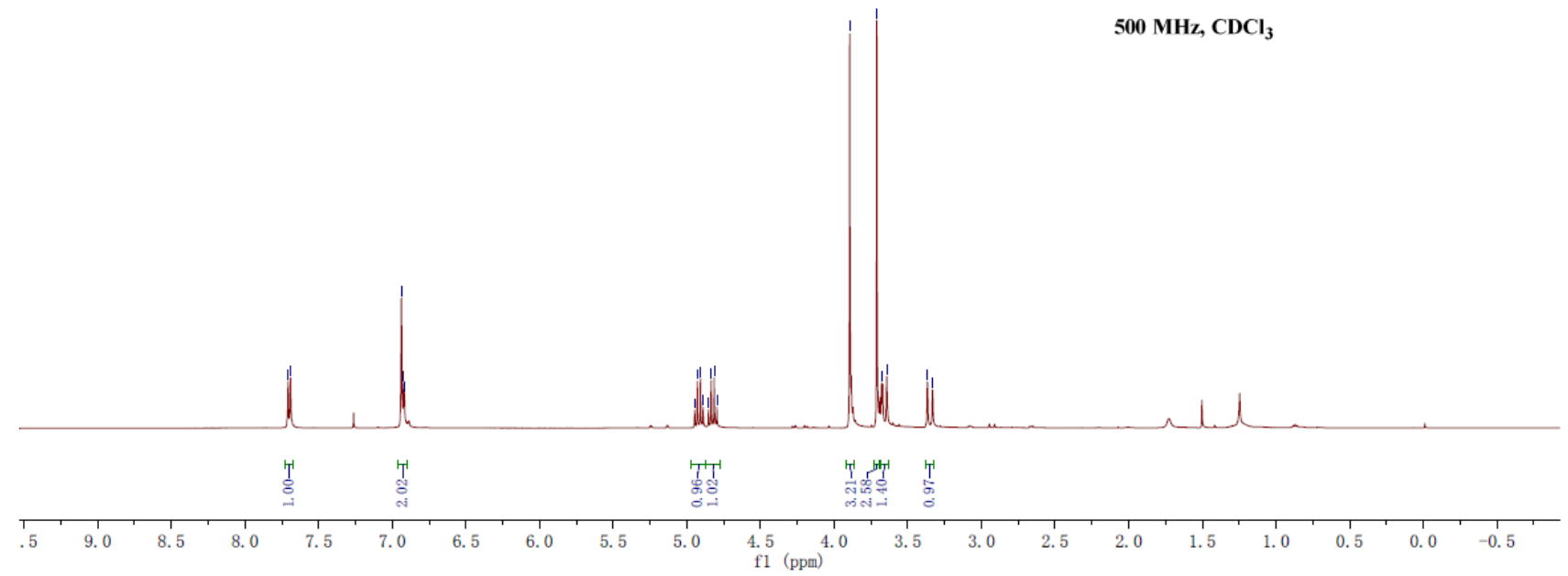




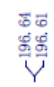

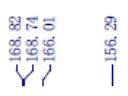

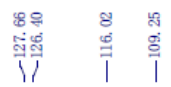

繁琵

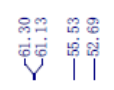

T.
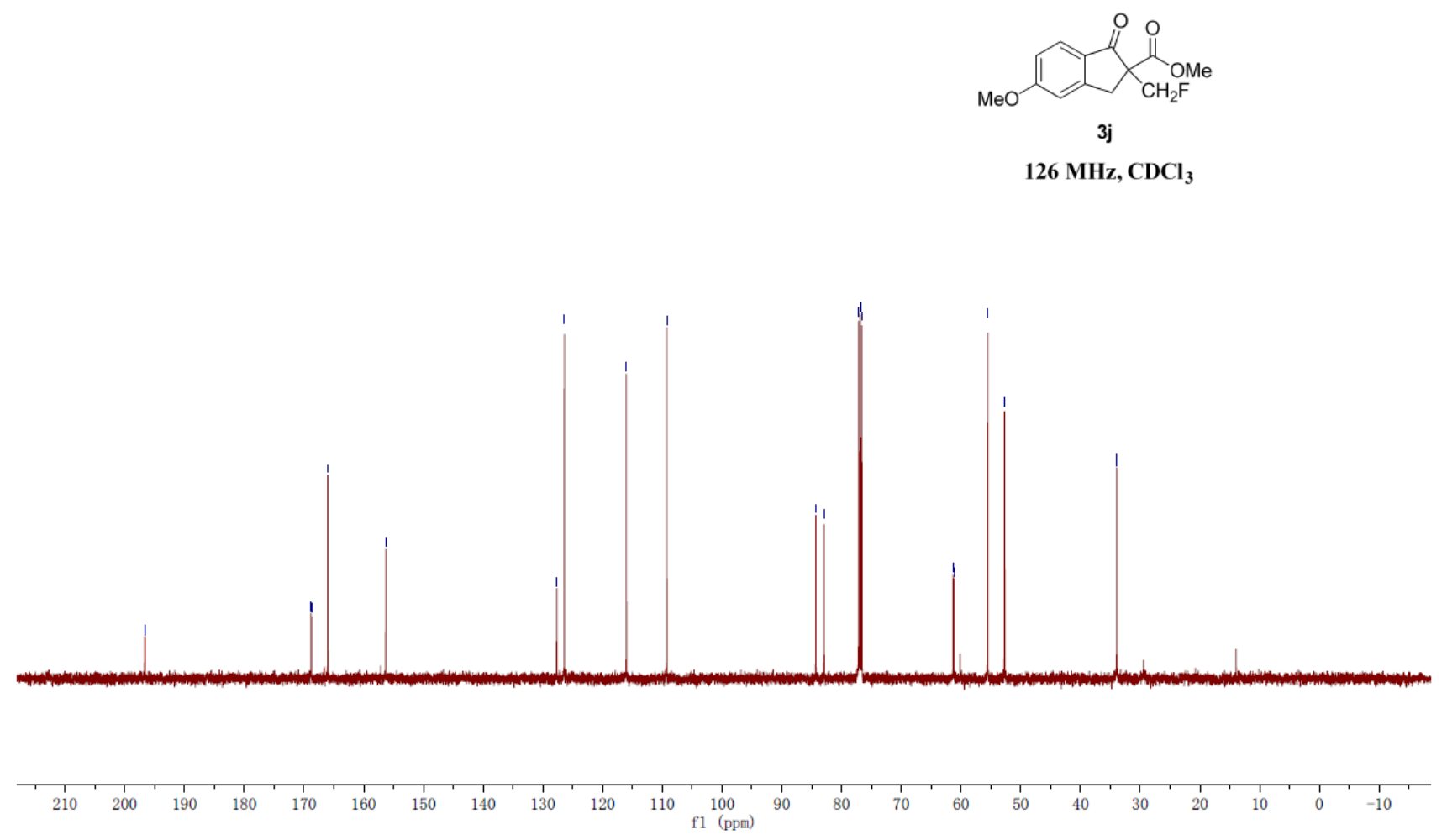

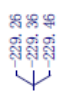

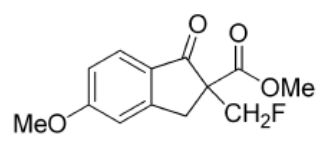

3j

$470 \mathrm{MHz}, \mathrm{CDCl}_{3}$

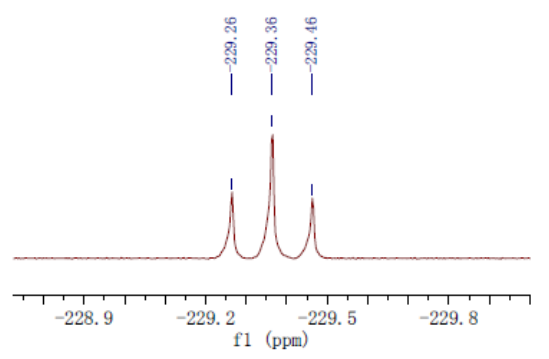

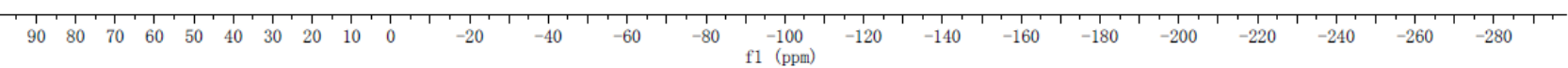

S80 

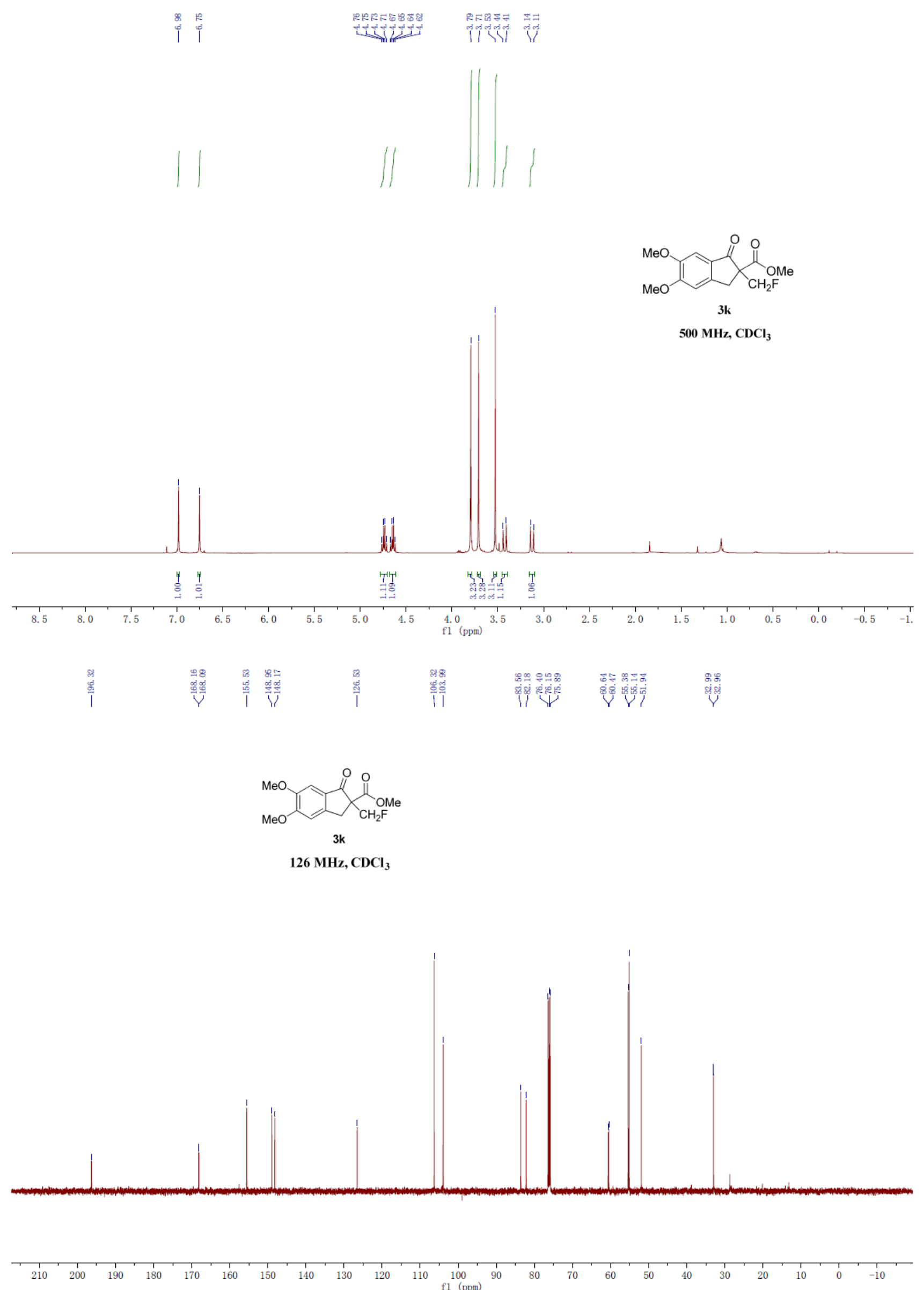


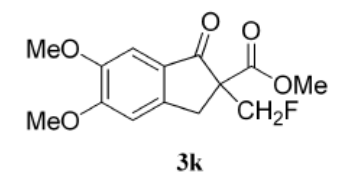

$470 \mathrm{MHz}, \mathrm{CDCl}_{3}$

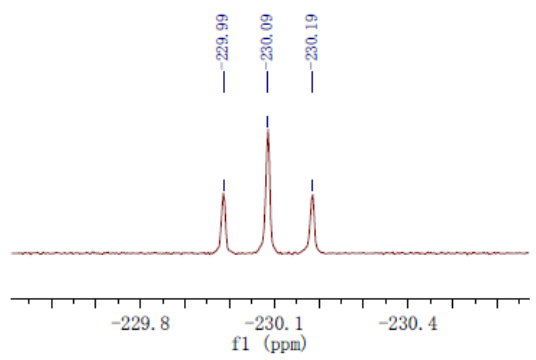

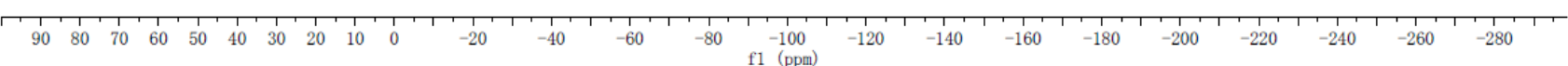

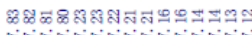

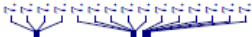

8.85न

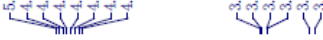

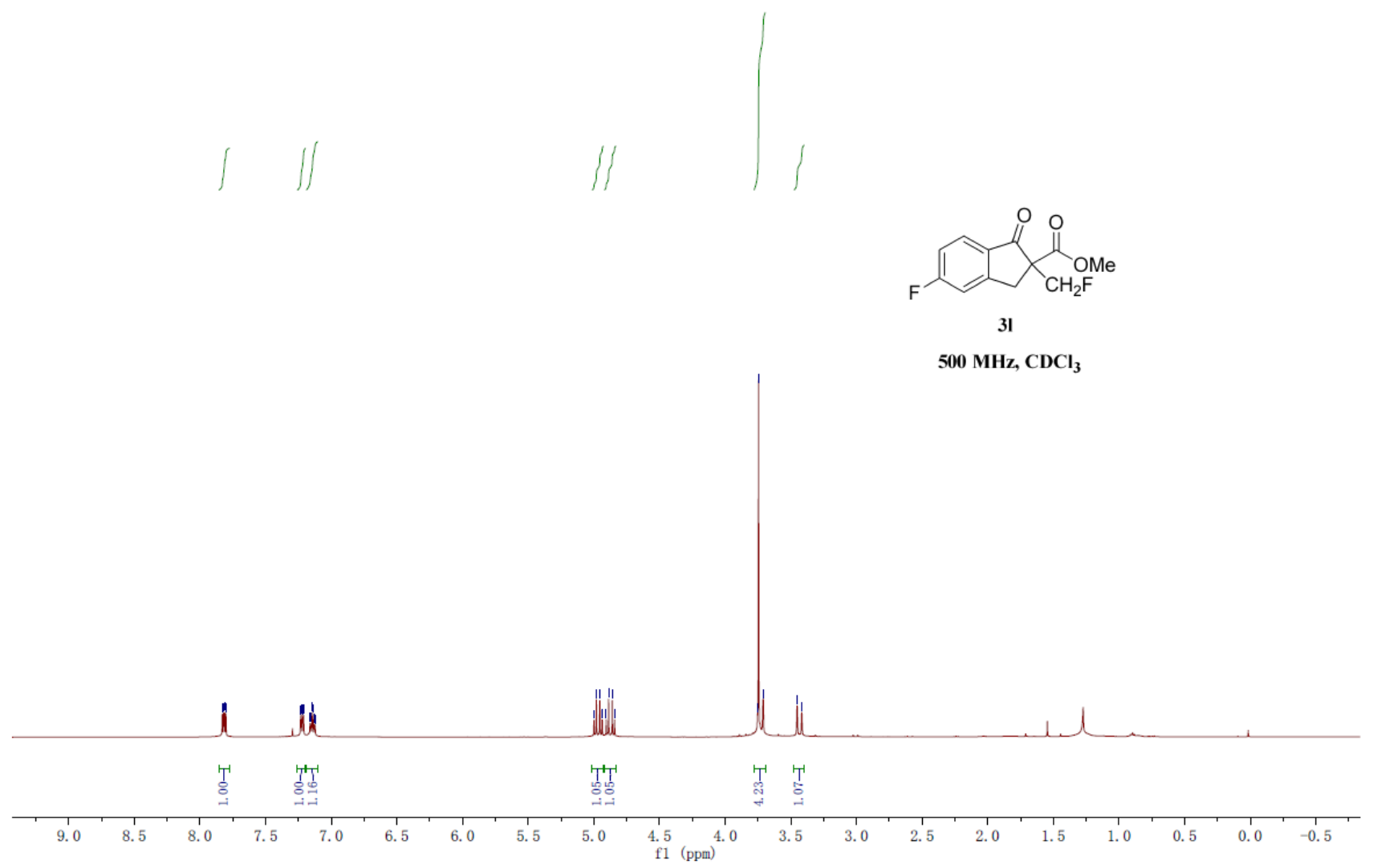




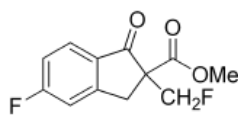

31

$126 \mathrm{MHz}, \mathrm{CDCl}_{3}$

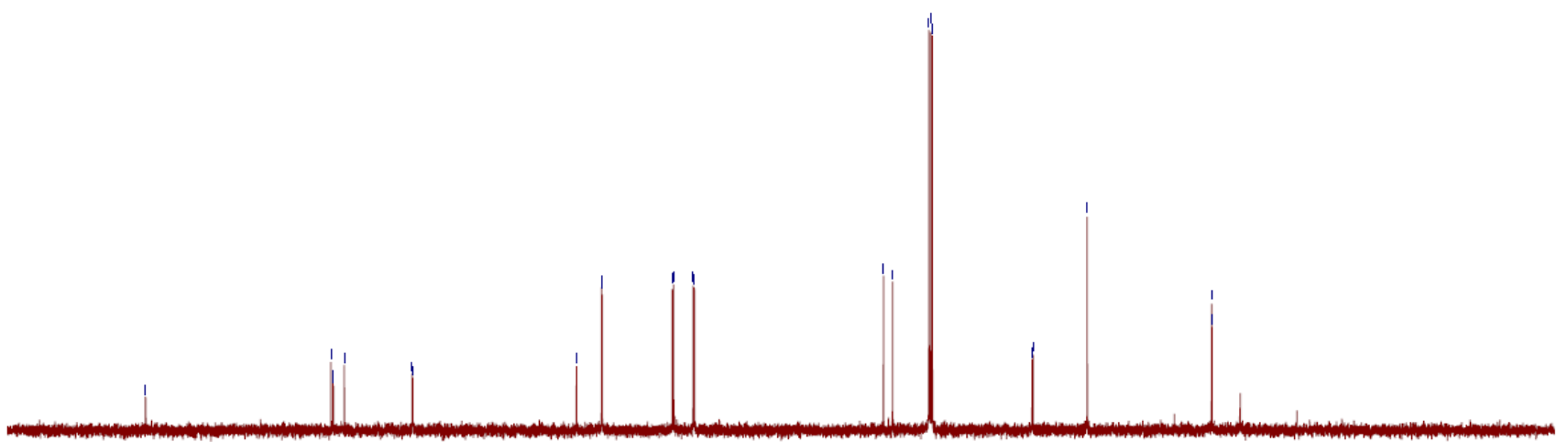

31

$470 \mathrm{MHz}, \mathrm{CDCl}_{3}$
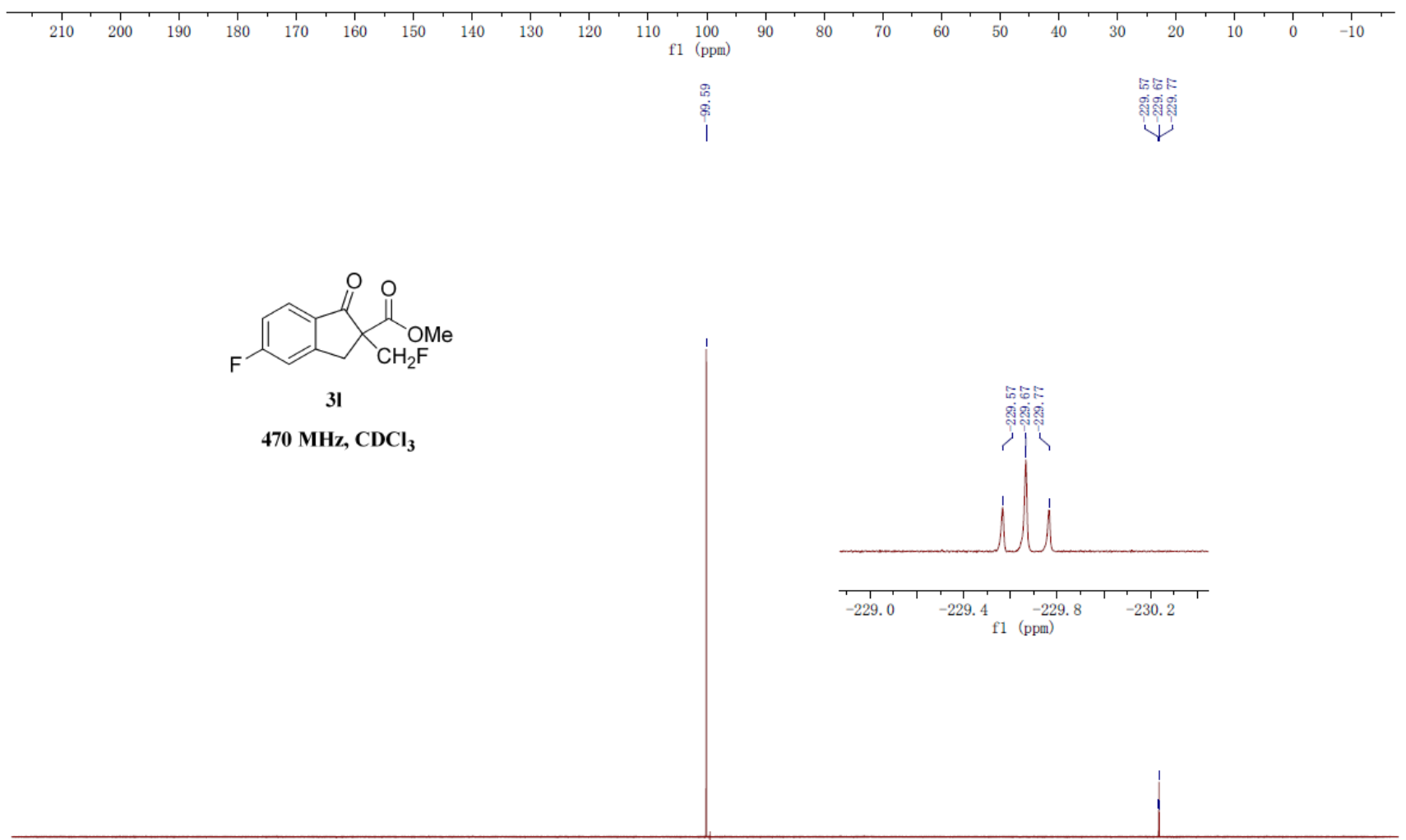

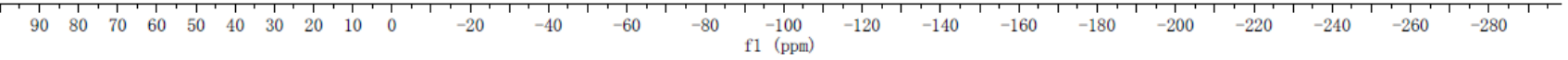



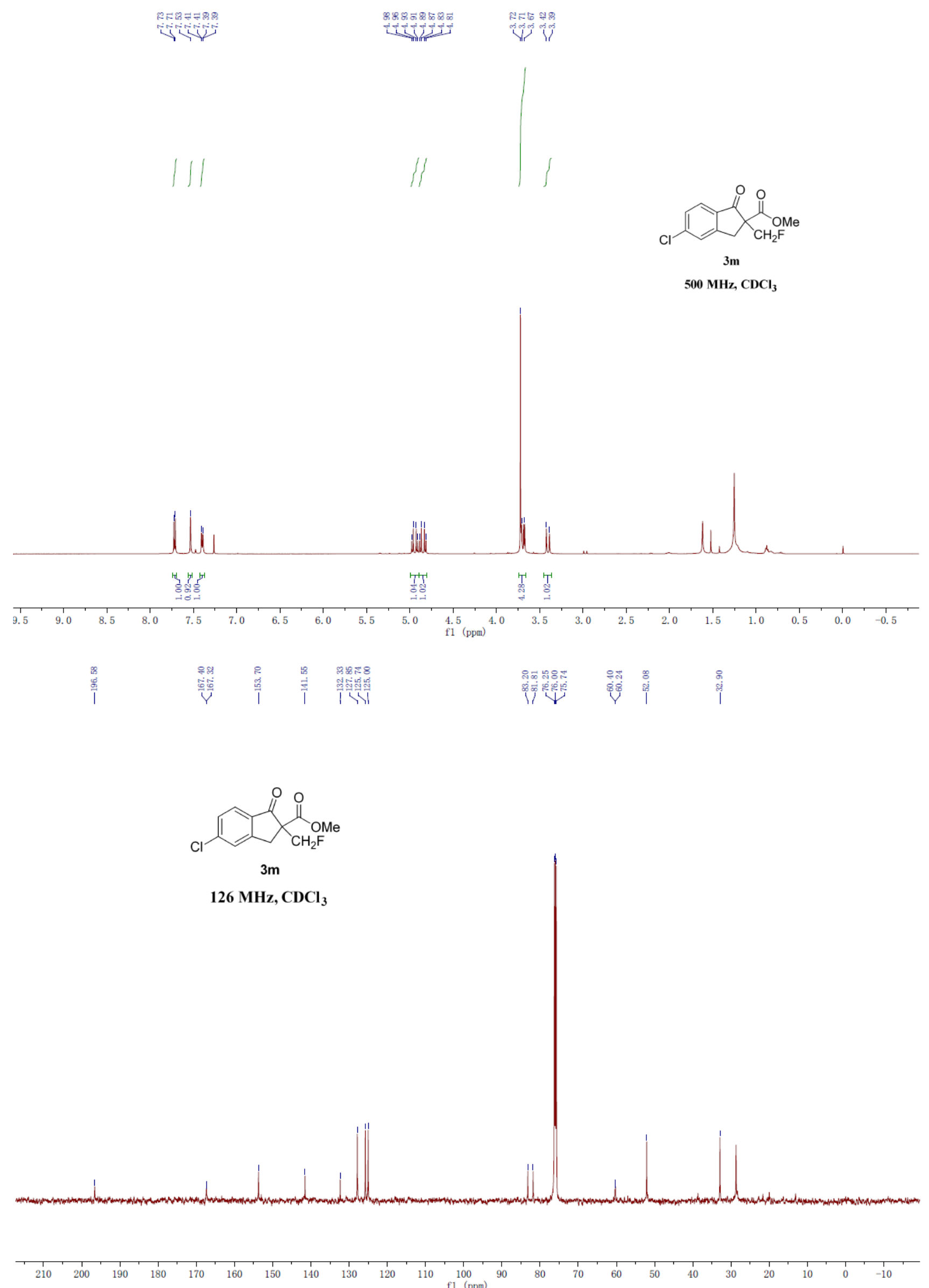


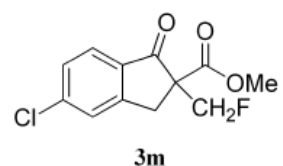

$470 \mathrm{MHz}, \mathrm{CDCl}_{3}$
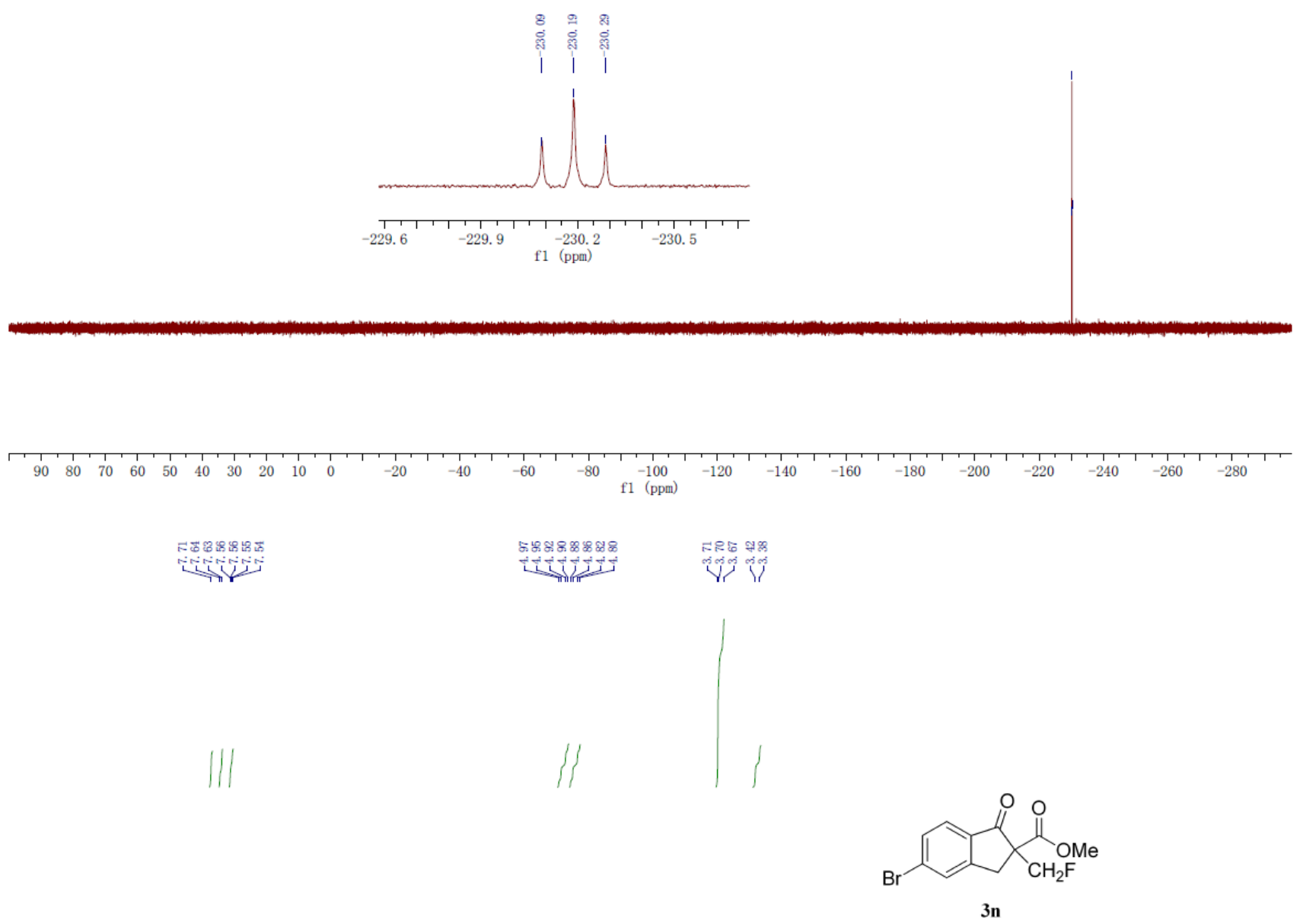

$500 \mathrm{MHz} \mathrm{CDCl}_{3}$

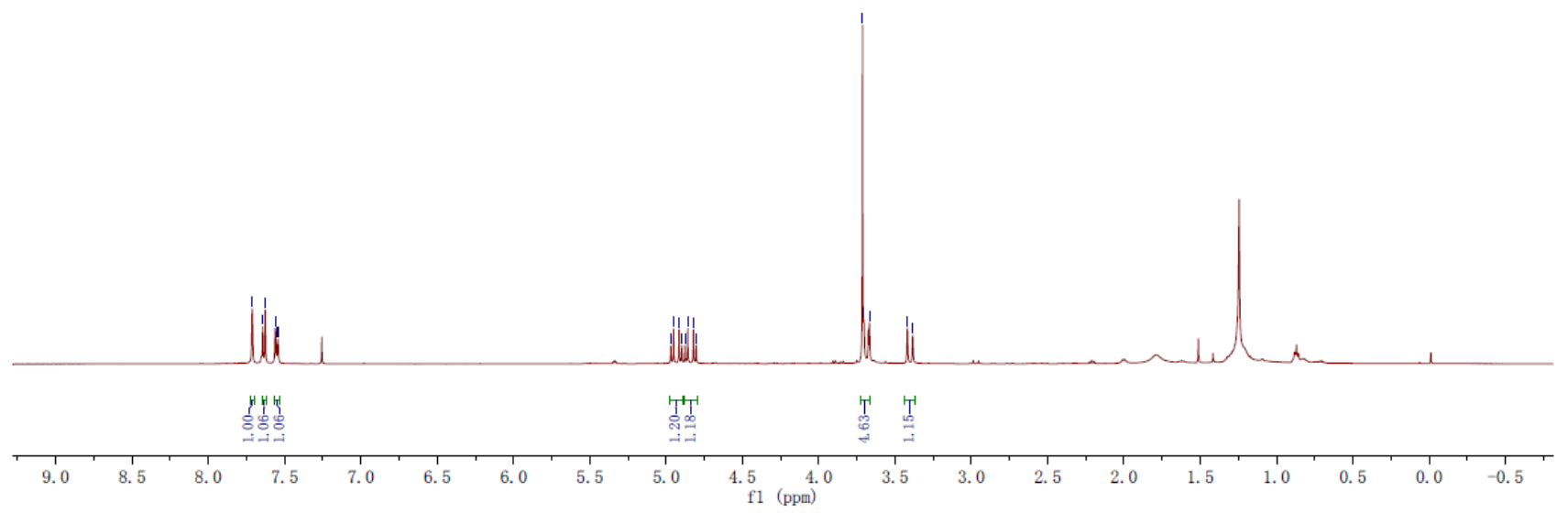




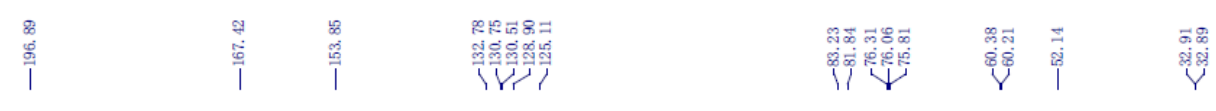

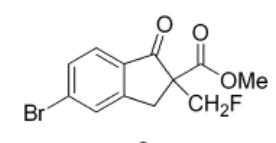

$3 n$

$126 \mathrm{MHz} \mathrm{CDCl}_{3}$
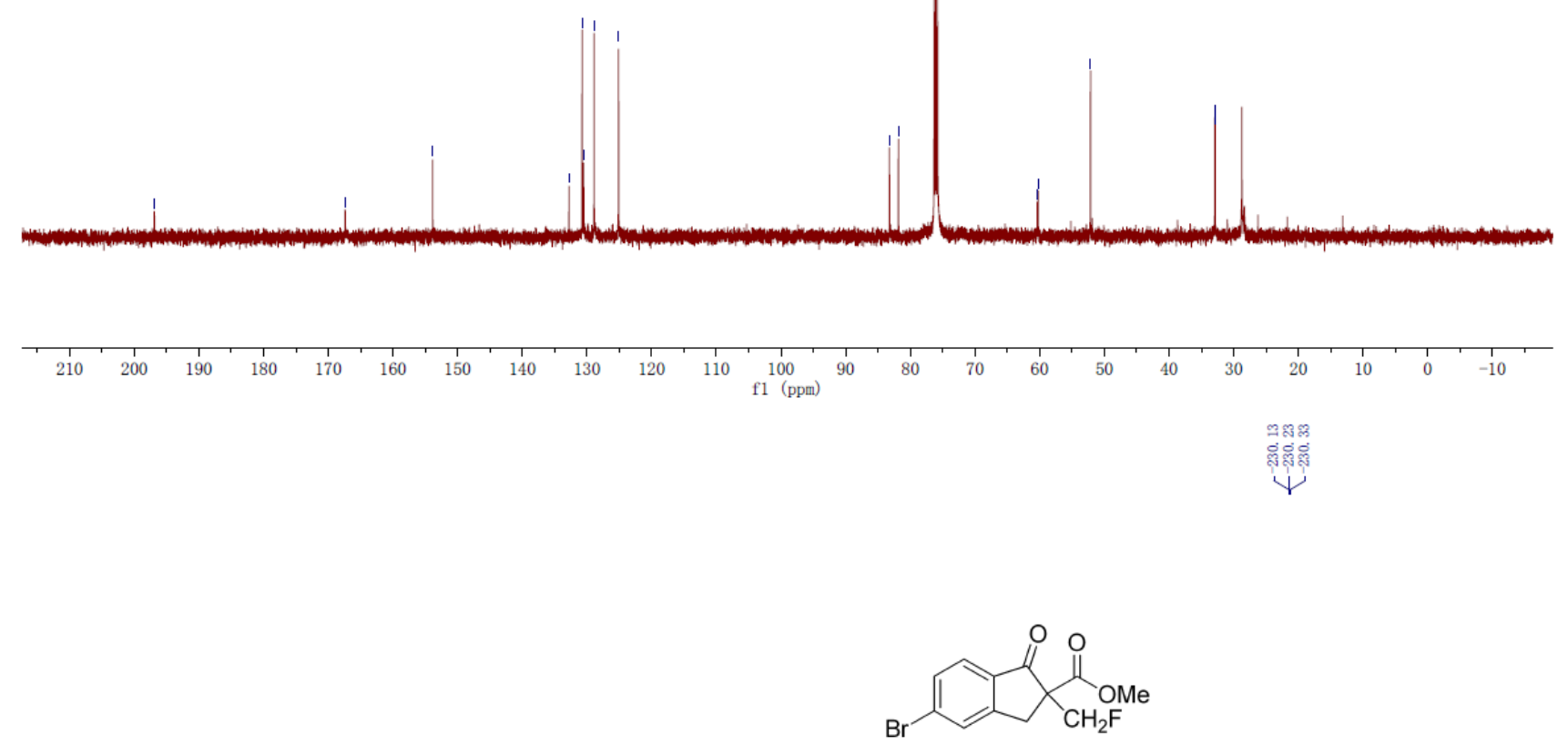

$3 n$

$470 \mathrm{MHz} \mathrm{CDCl}_{3}$

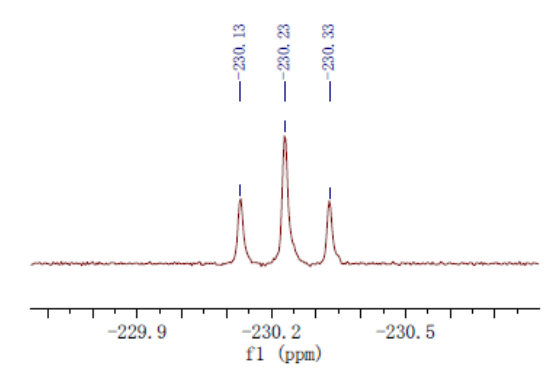

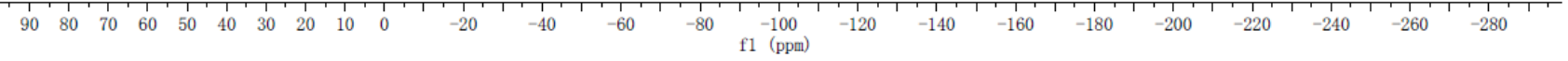




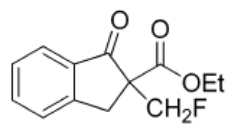

30

$500 \mathrm{MHz} \mathrm{CDCl}_{3}$

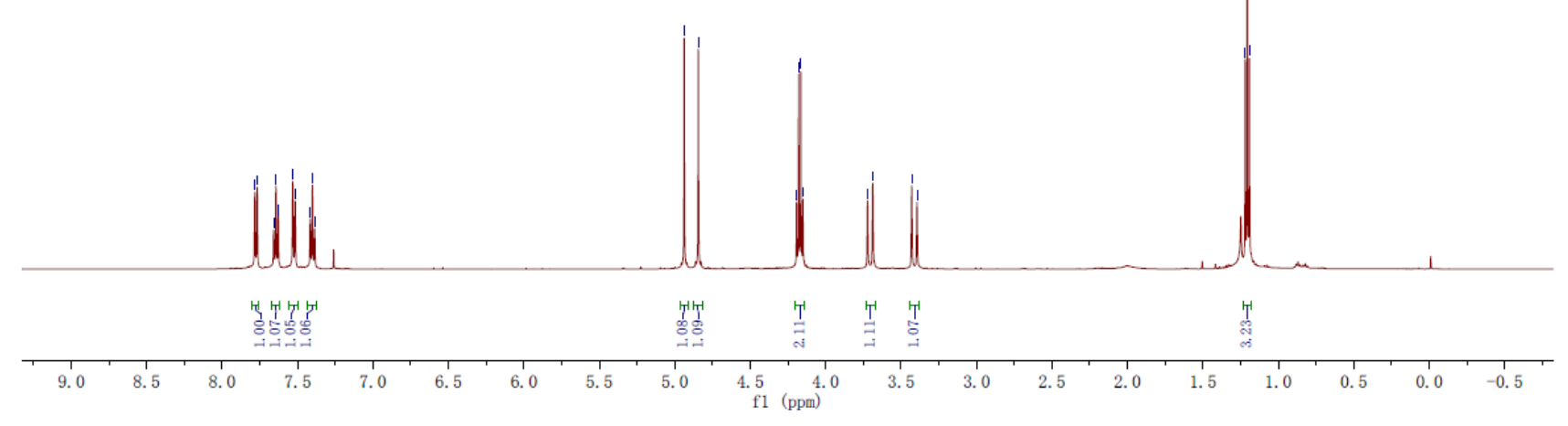

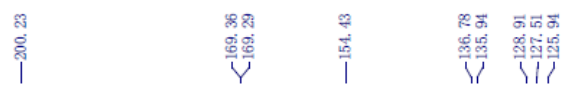

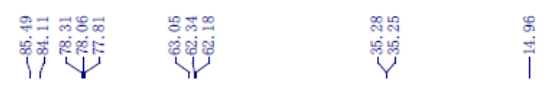

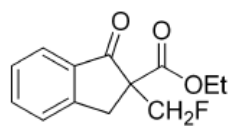

30

$126 \mathrm{MHz} \mathrm{CDCl}_{3}$
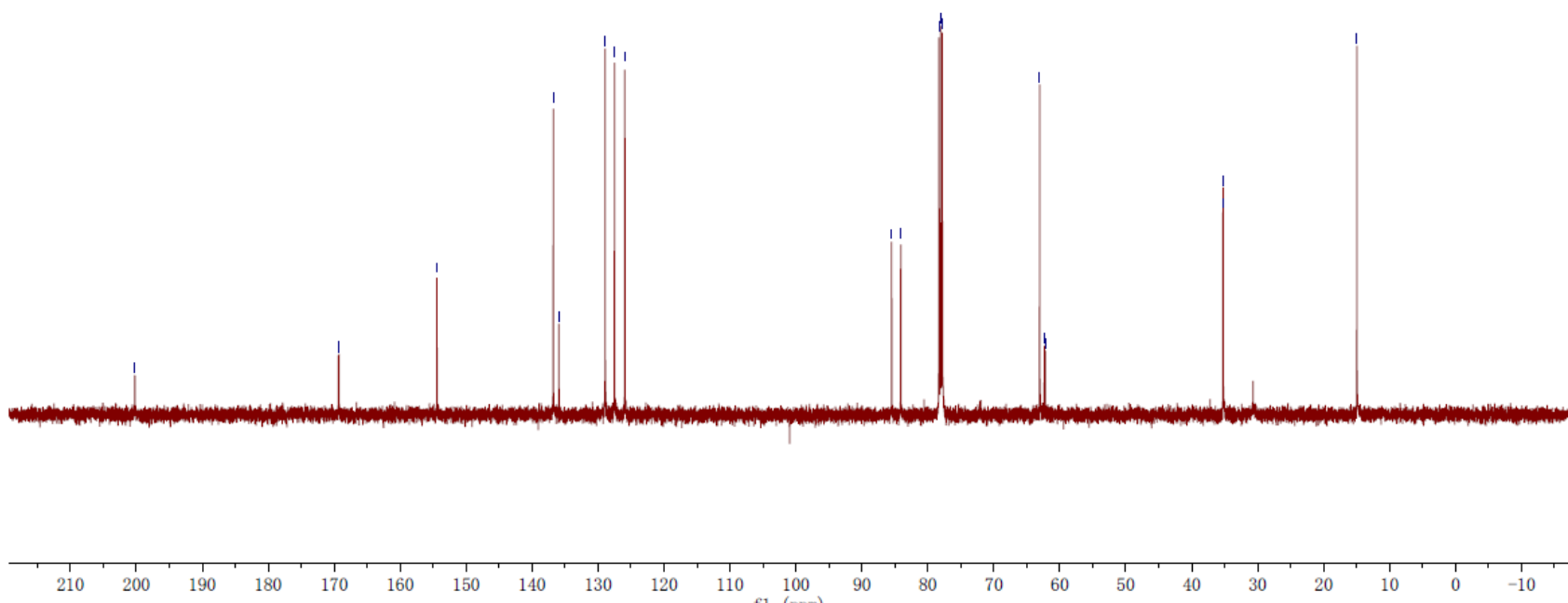


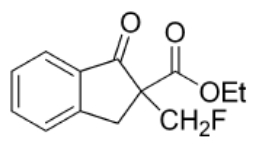

30

$470 \mathrm{MHz}, \mathrm{CDCl}_{3}$
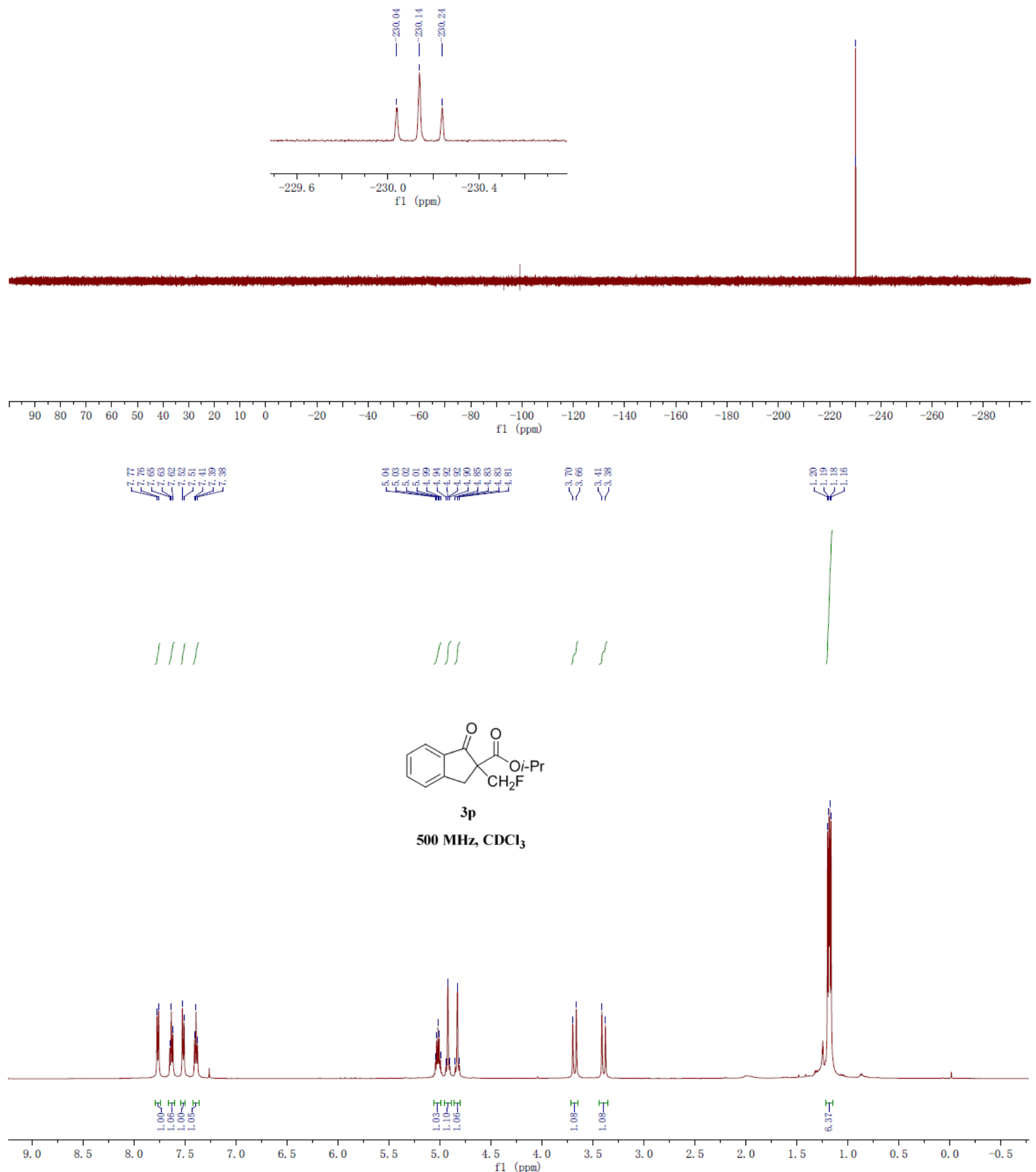
$\stackrel{\frac{12}{9}}{\frac{9}{1}}$

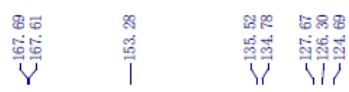

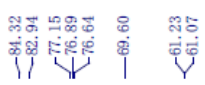

พั

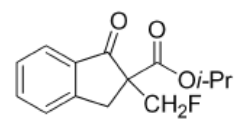

$3 p$

$126 \mathrm{MHz} \mathrm{CDCl}_{3}$
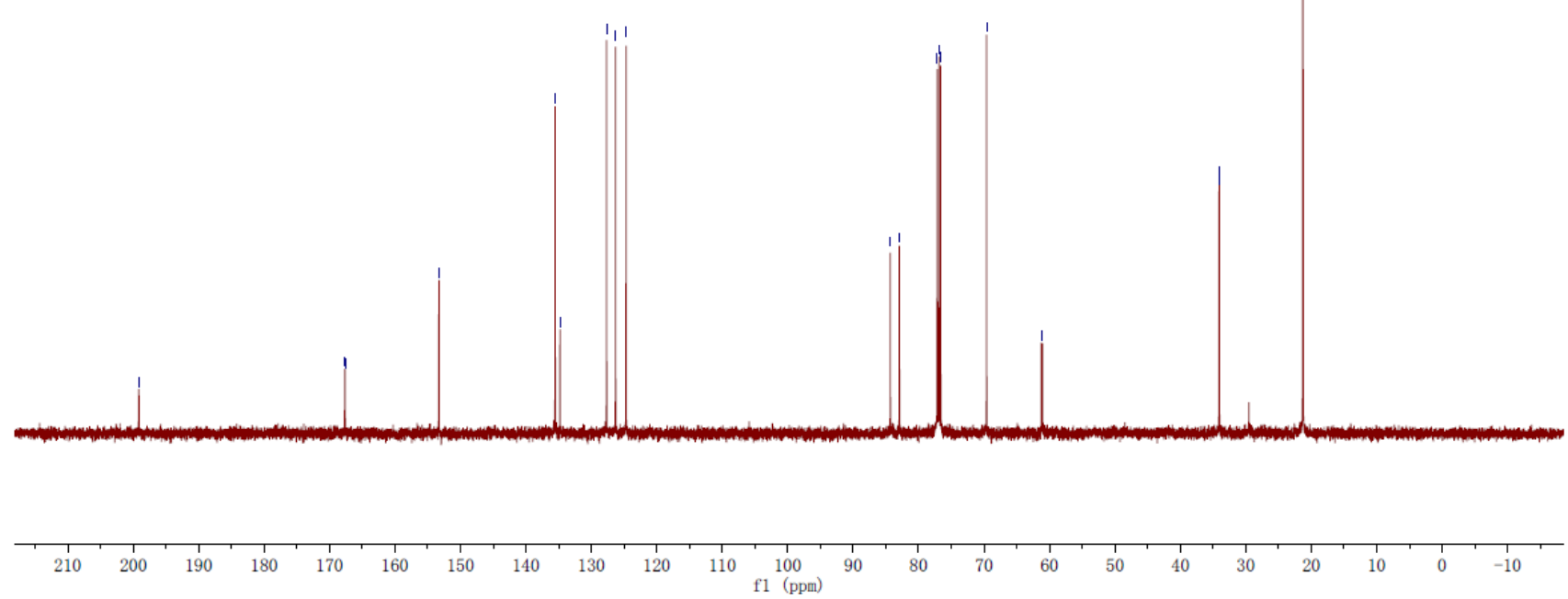

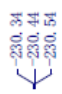

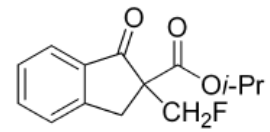

3p

$470 \mathrm{MHz}, \mathrm{CDCl}_{3}$
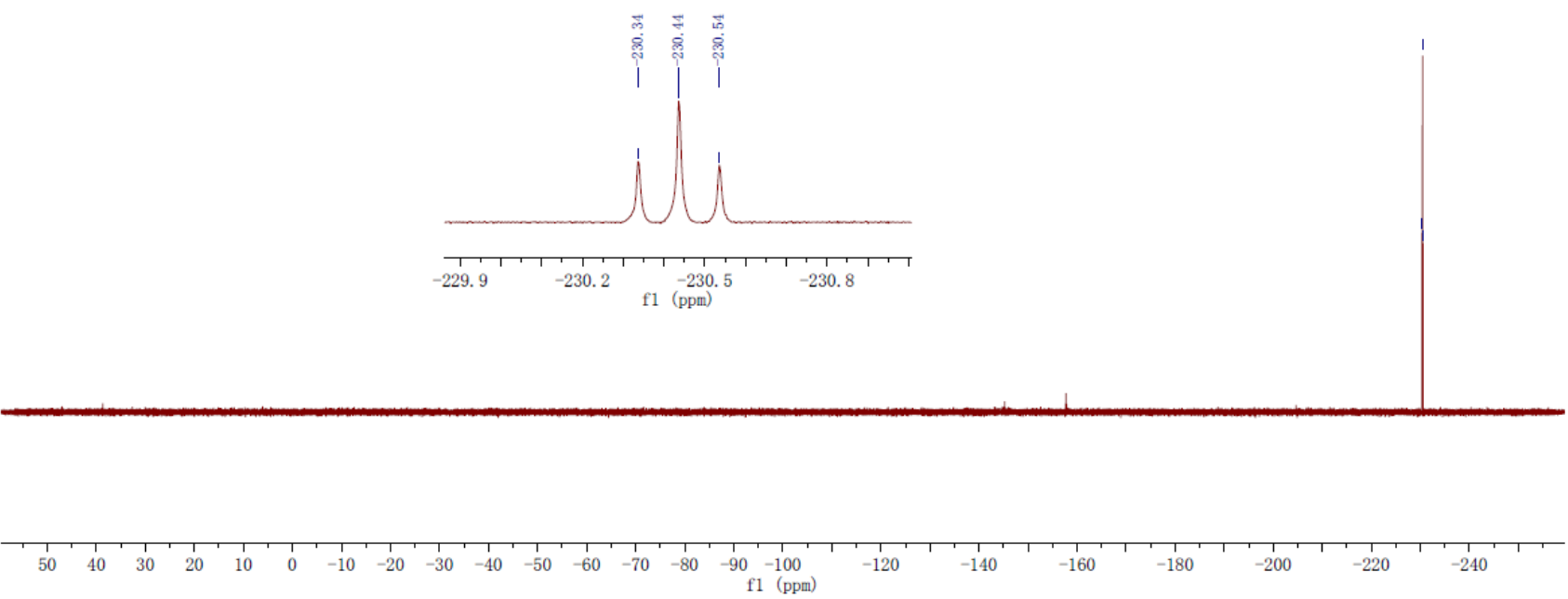

S89 


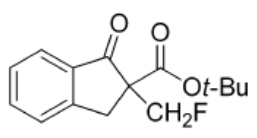

$3 q$

$500 \mathrm{MHz} \mathrm{CDCl}_{3}$

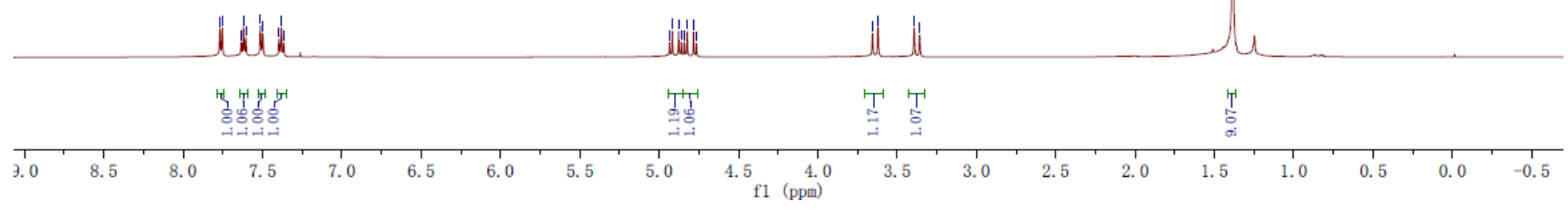

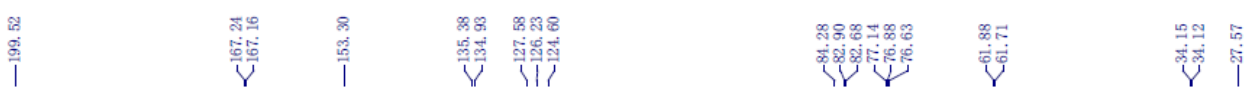

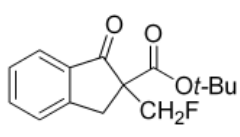

$3 q$

$126 \mathrm{MHz} \mathrm{CDCl}_{3}$
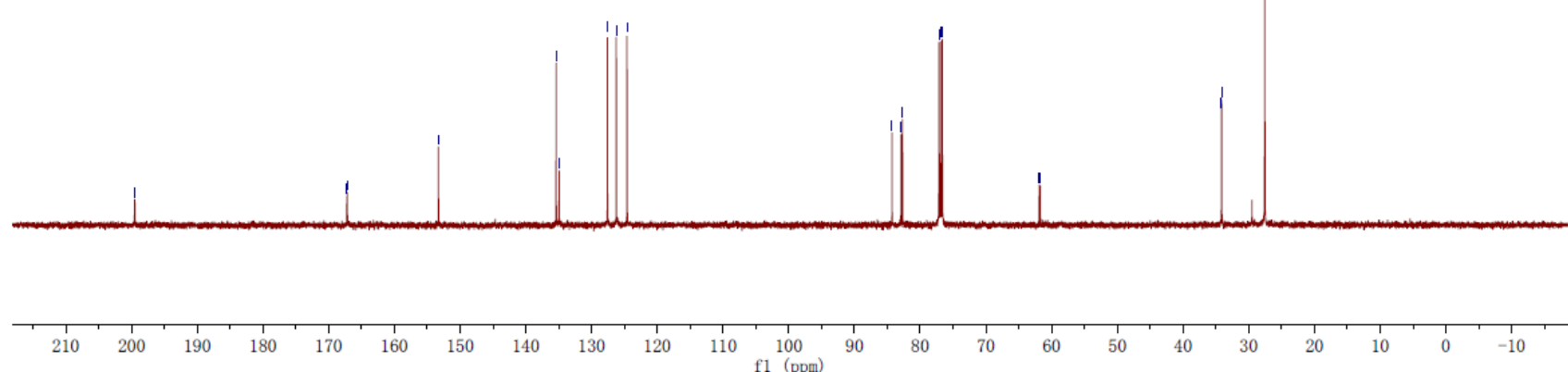


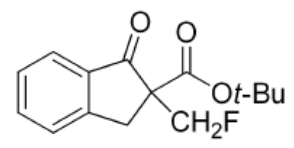

$3 q$

$470 \mathrm{MHz}, \mathrm{CDCl}_{3}$

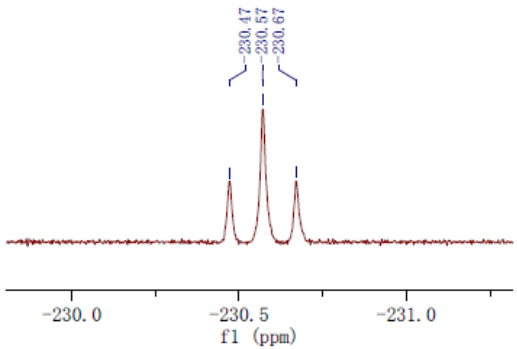

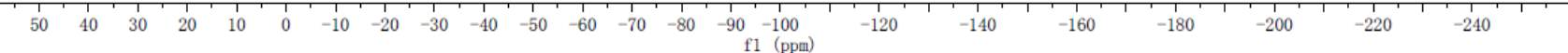

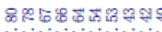
intivitising

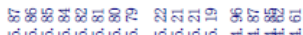

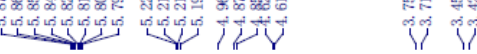
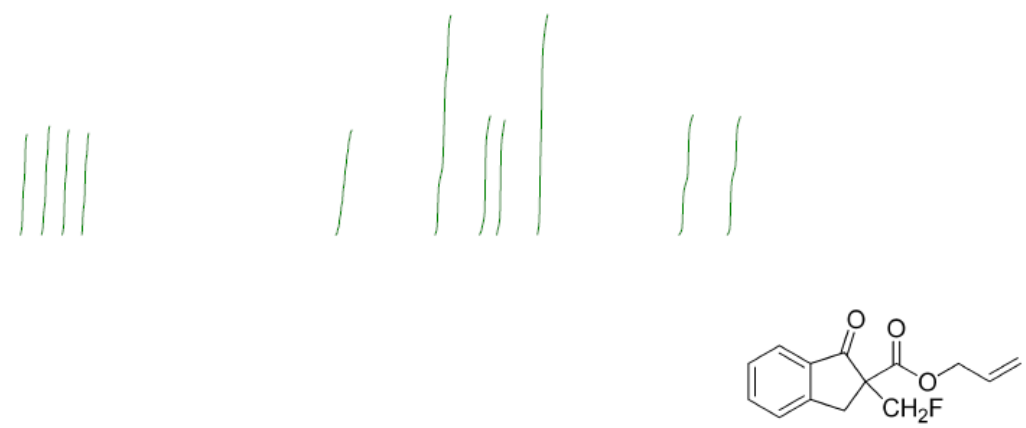

$3 \mathbf{r}$

$500 \mathrm{MHz}, \mathrm{CDCl}_{3}$

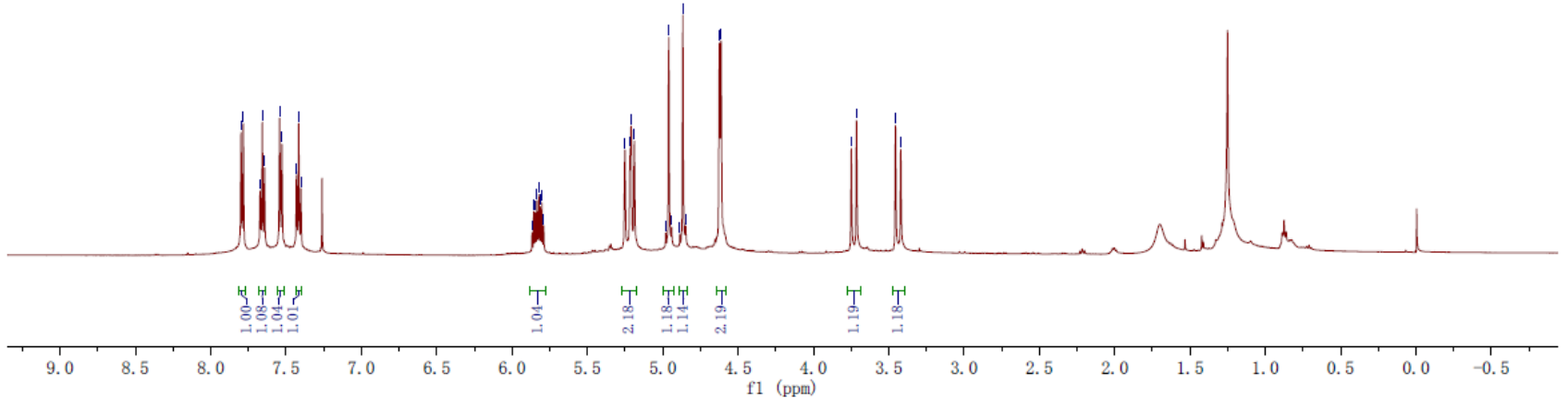




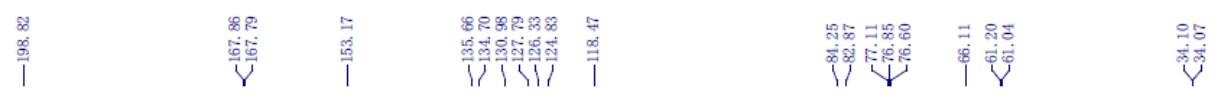

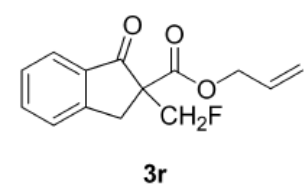

$126 \mathrm{MHz}, \mathrm{CDCl}_{3}$

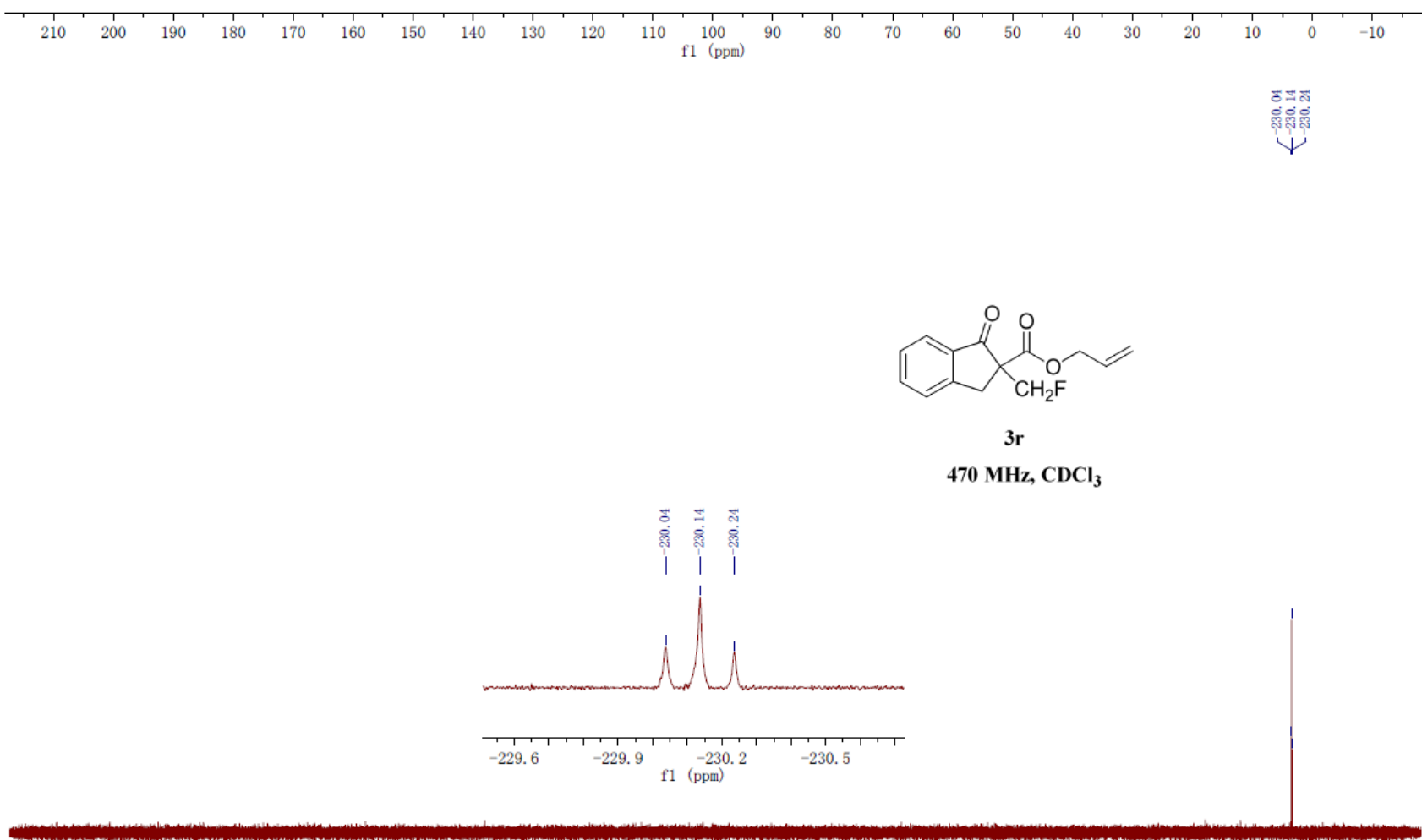

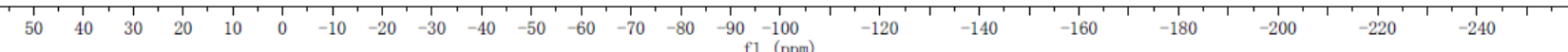



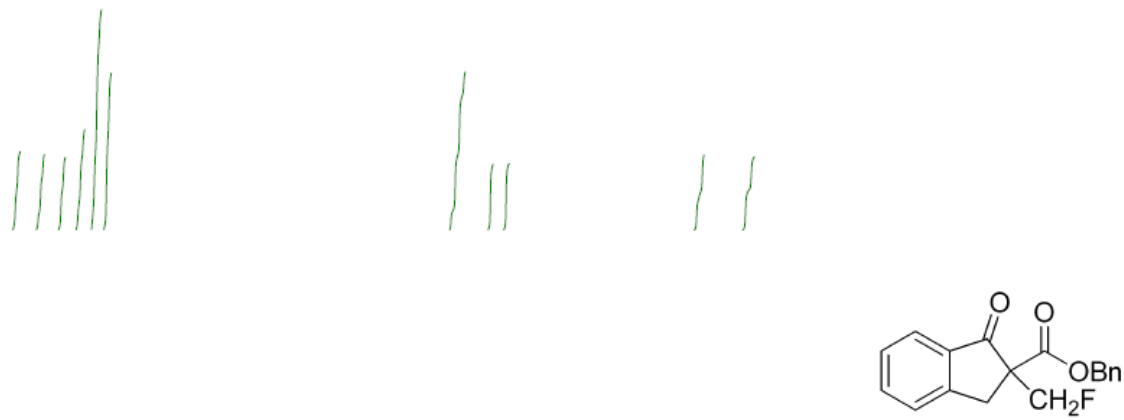

$3 s$

$500 \mathrm{MHz}, \mathrm{CDCl}_{3}$
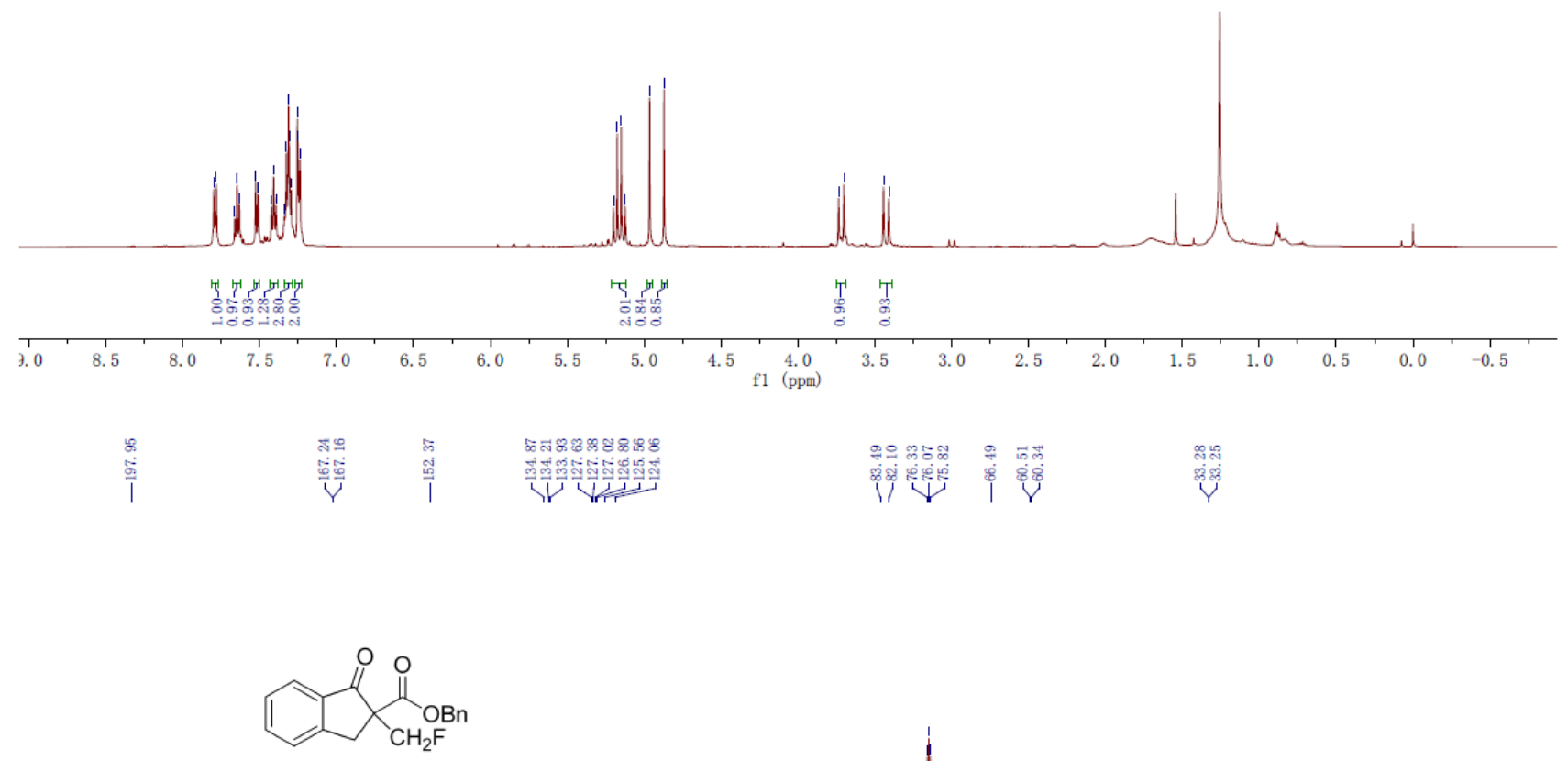

3s

$126 \mathrm{MHz} \mathrm{CDCl}_{3}$

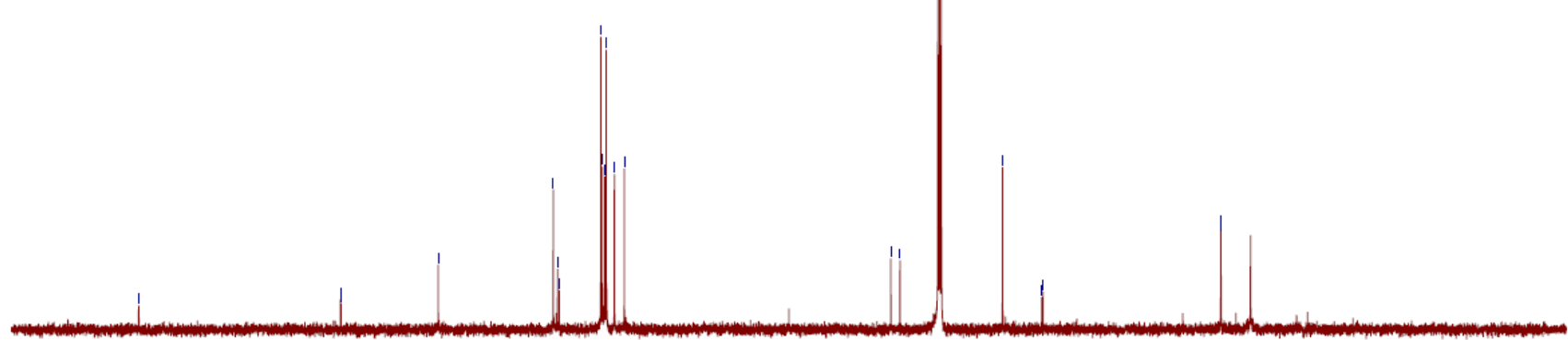




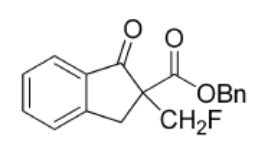

3s

$470 \mathrm{MHz}, \mathrm{CDCl}_{3}$
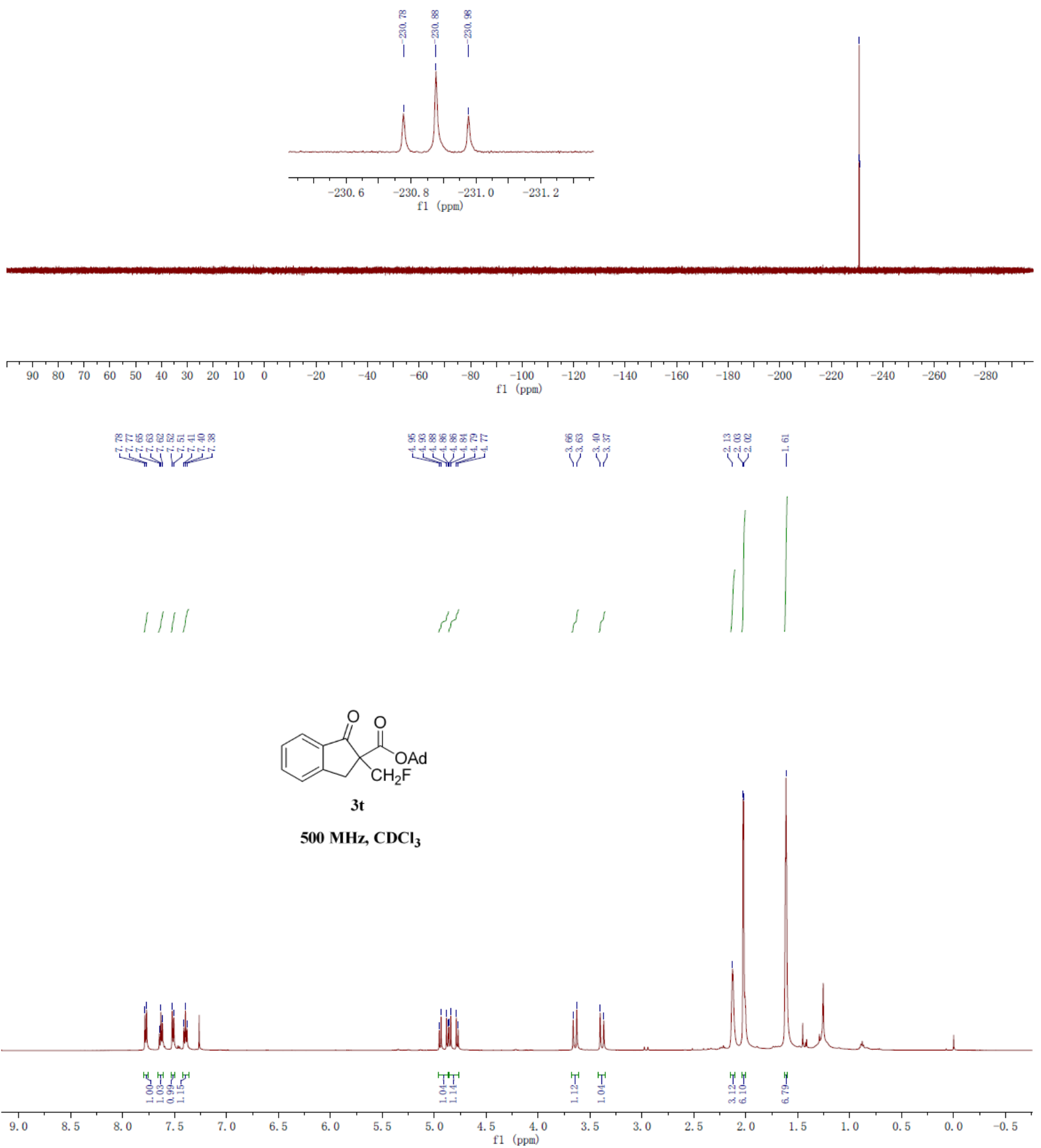


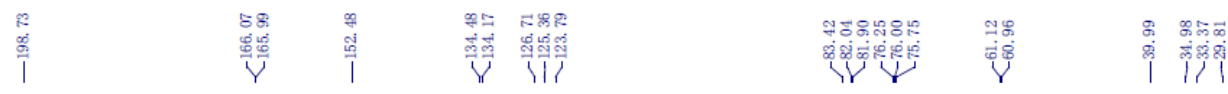

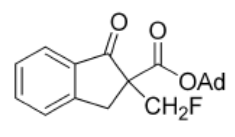

$3 \mathrm{t}$

$126 \mathrm{MHz}, \mathrm{CDCl}_{3}$
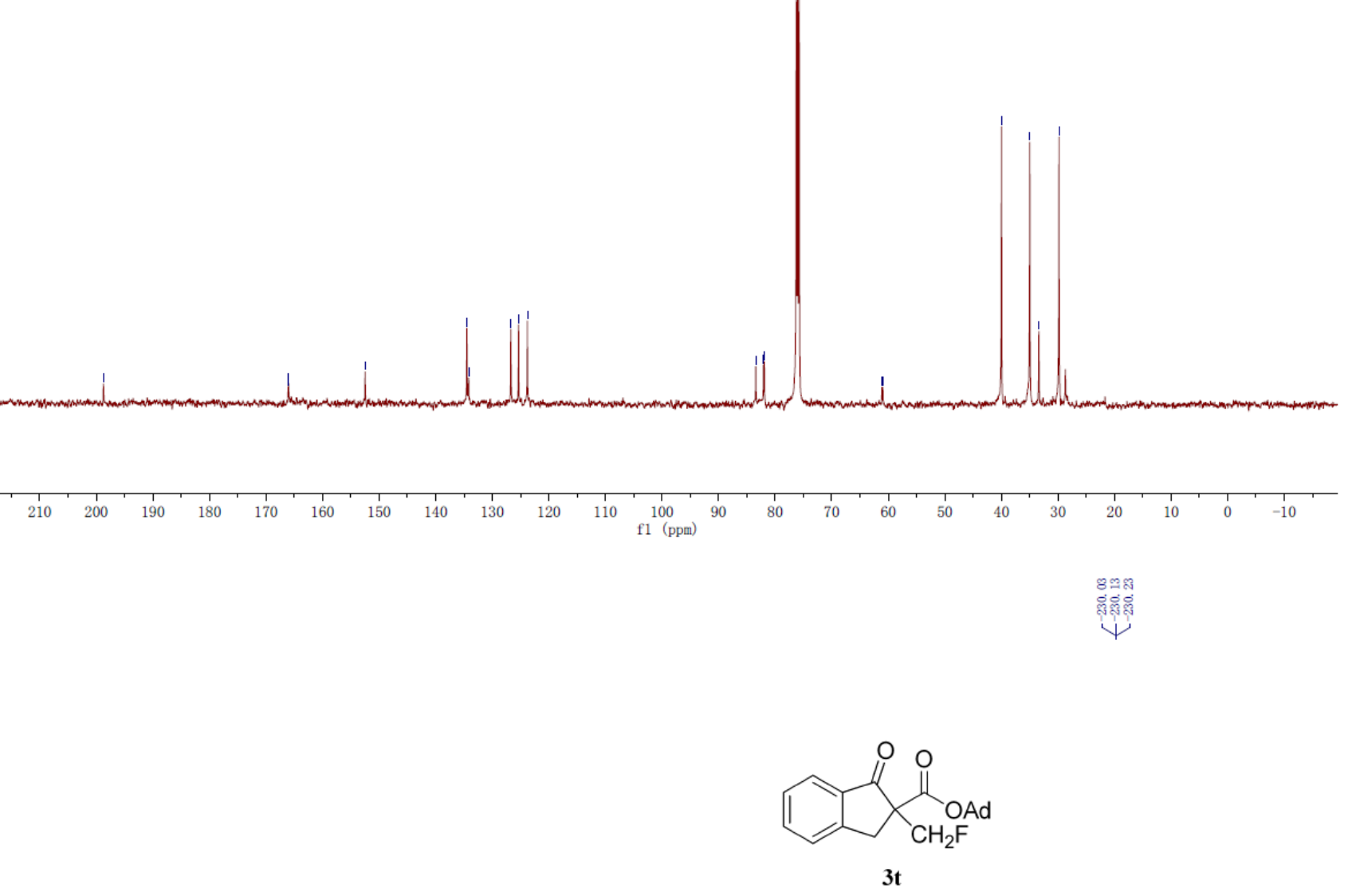

$470 \mathrm{MHz}, \mathrm{CDCl}_{3}$

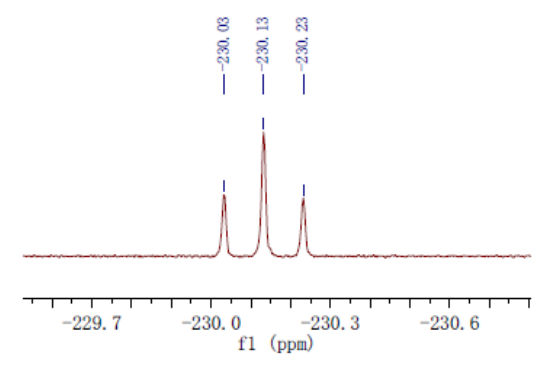

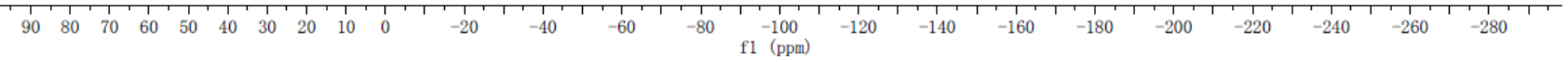




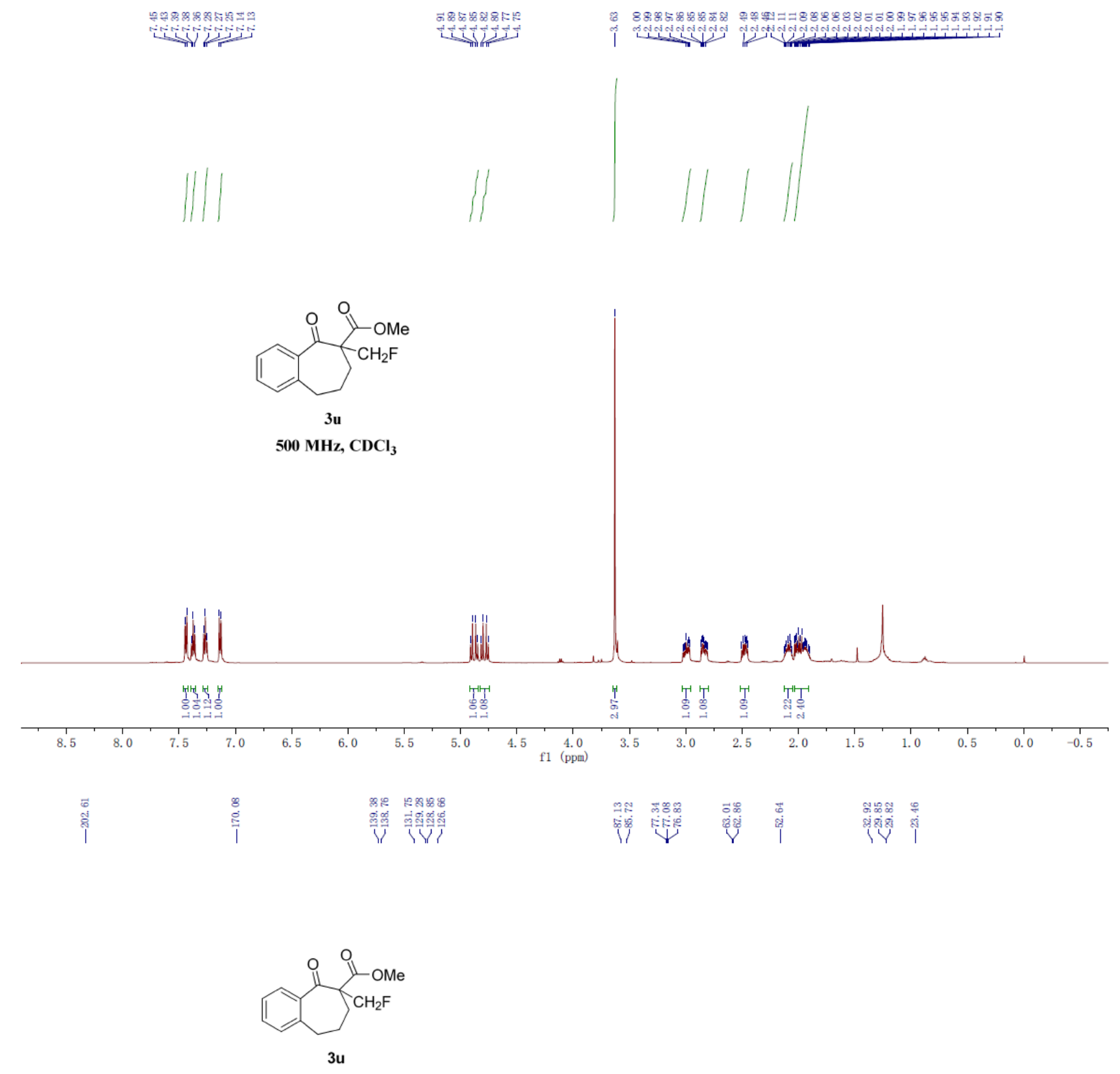

$126 \mathrm{MHz}, \mathrm{CDCl}_{3}$

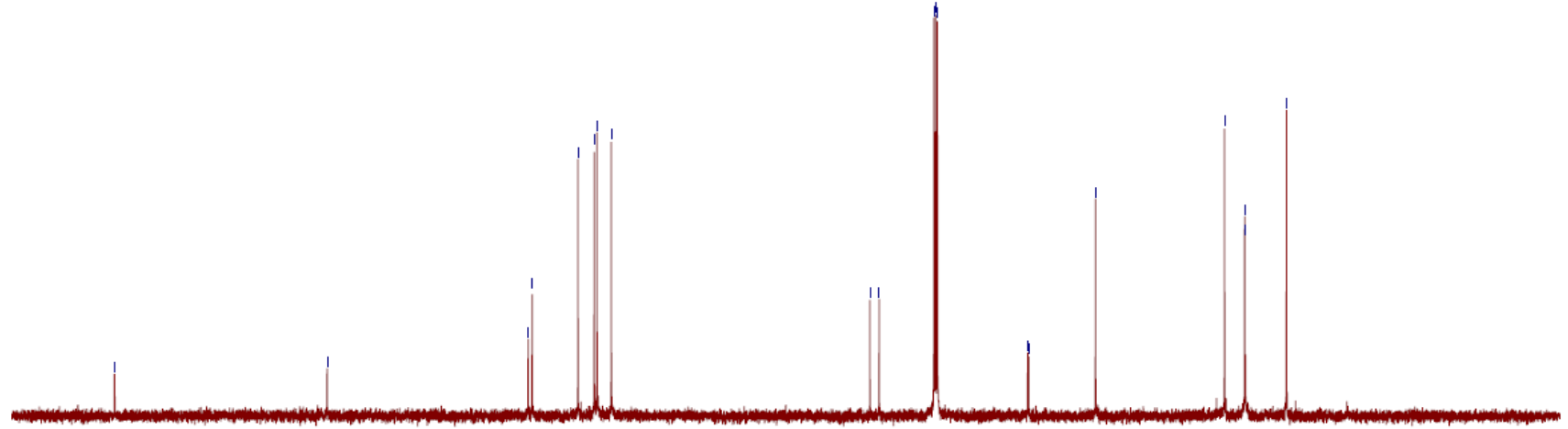

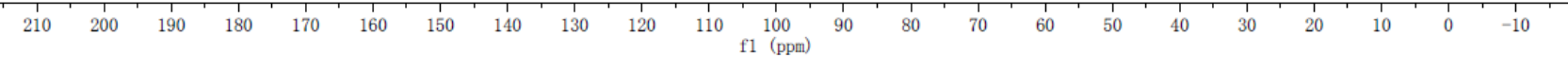




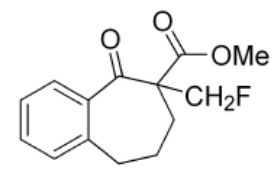

3u

$470 \mathrm{MHz}, \mathrm{CDCl}_{3}$

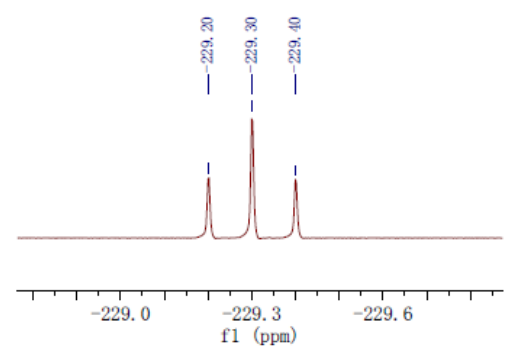

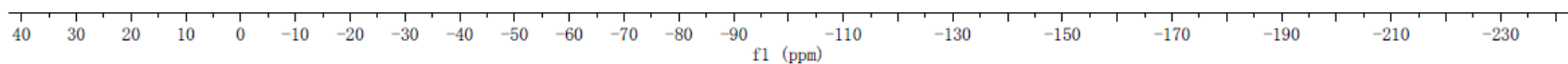

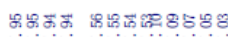

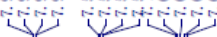

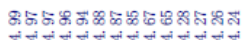

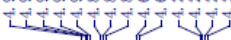

为年
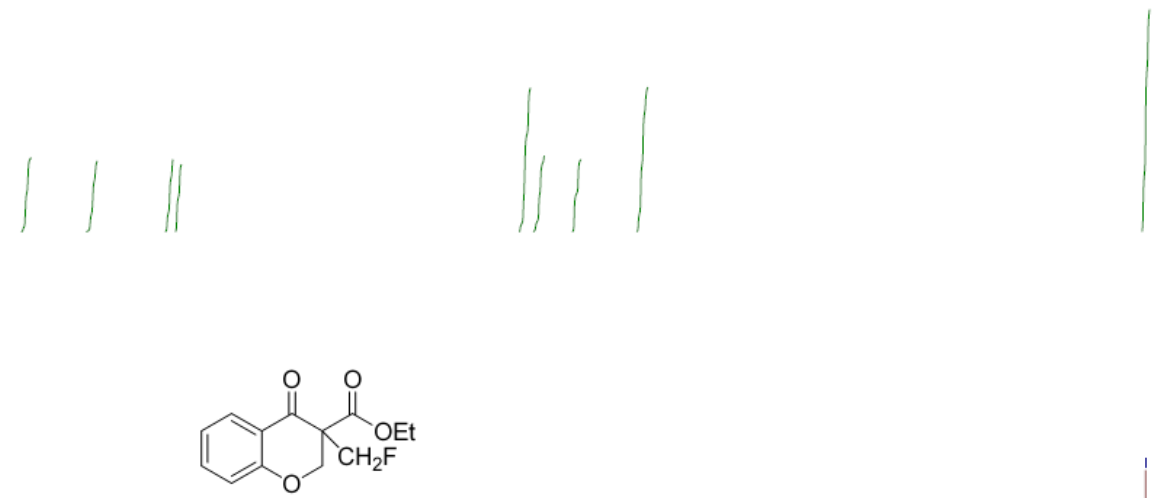

$3 v$

$500 \mathrm{MHz}, \mathrm{CDCl}_{3}$

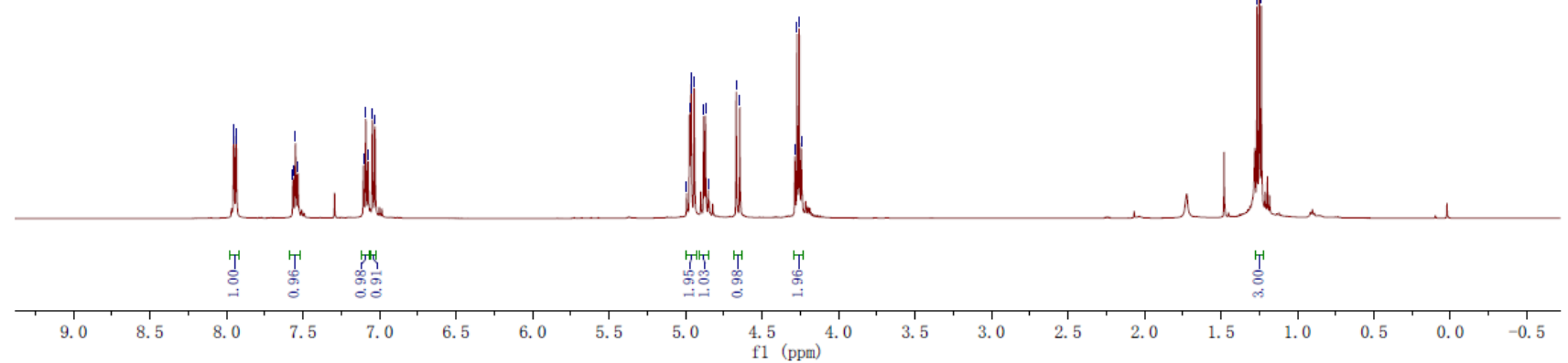




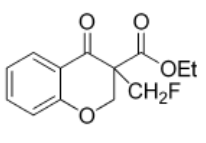

$126 \mathrm{MHz}, \mathrm{CDCl}_{3}$
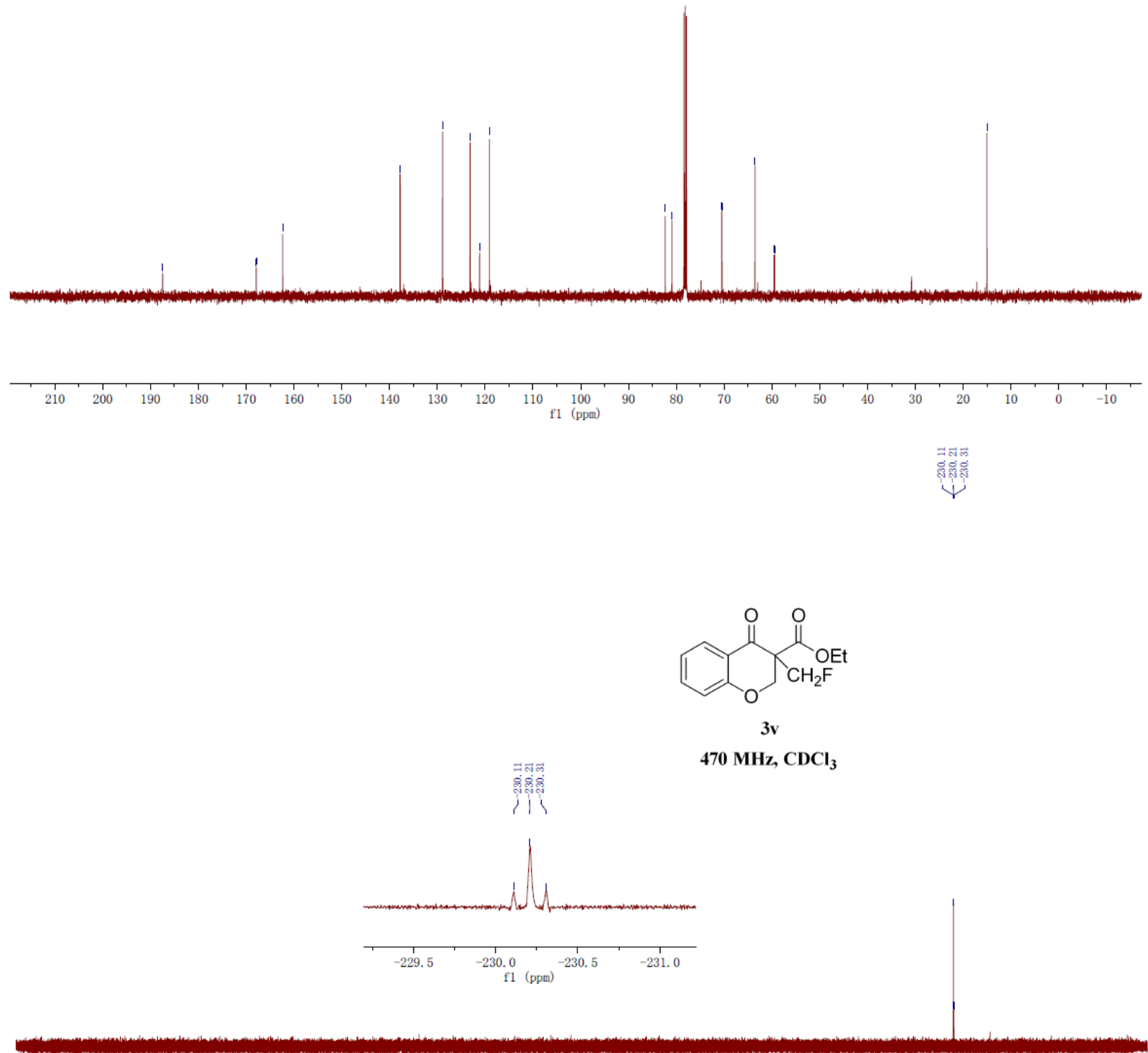

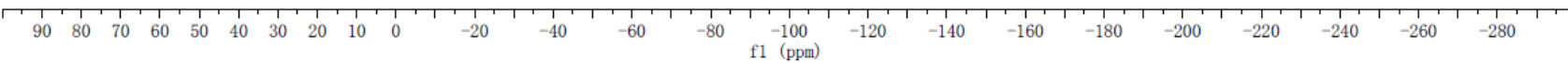




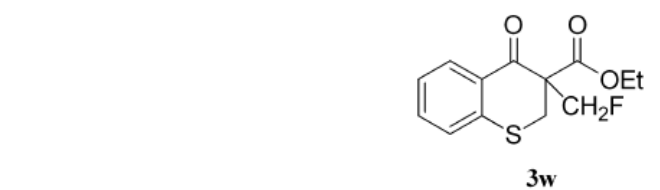

$500 \mathrm{MHz} \mathrm{CDCl}_{3}$

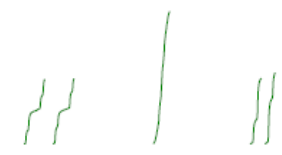

$500 \mathrm{MHz}, \mathrm{CDCl}_{3}$

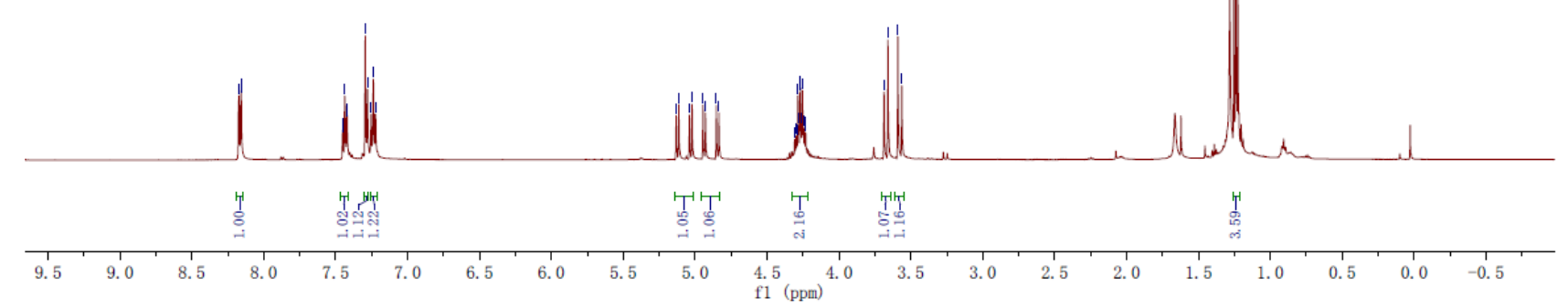

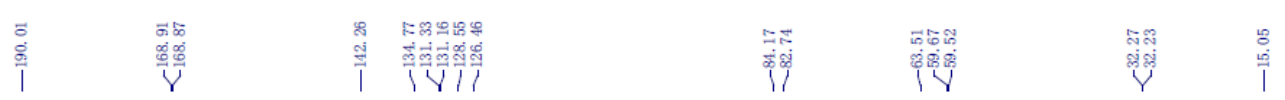

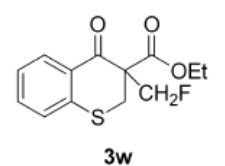

$126 \mathrm{MHz} \mathrm{CDCl}_{3}$
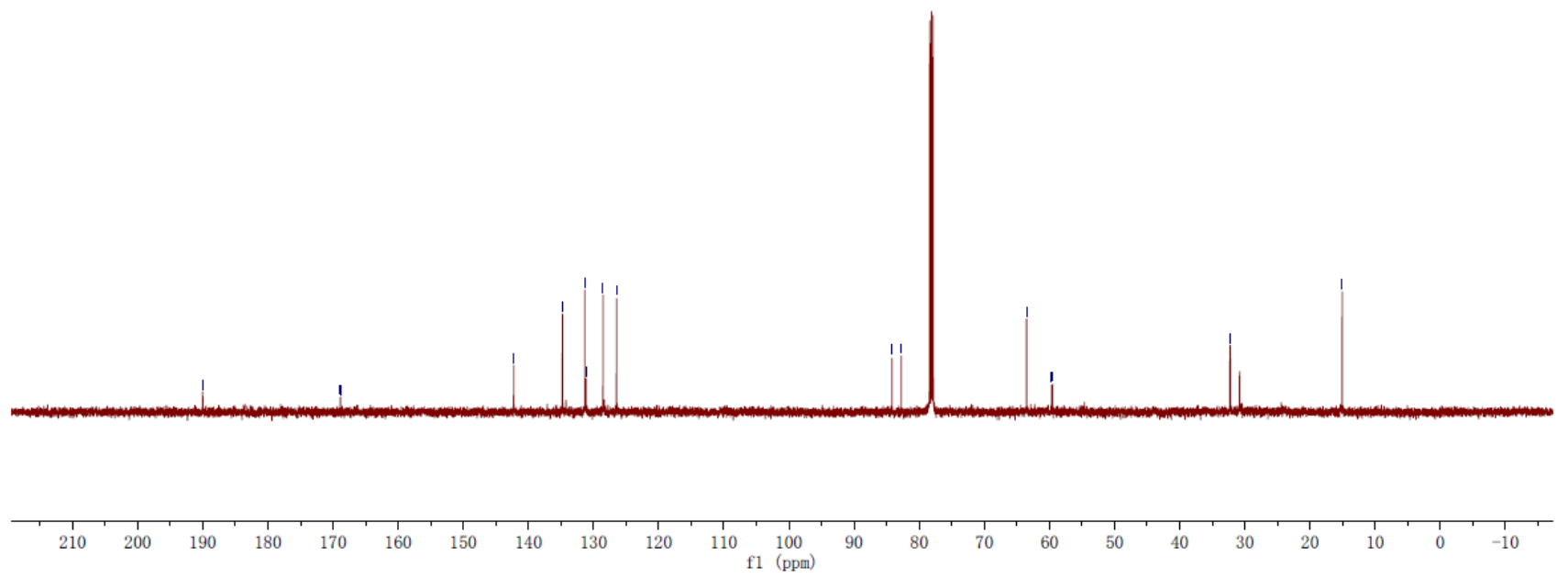


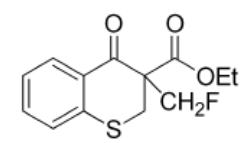

$3 w$

$470 \mathrm{MHz}, \mathrm{CDCl}_{3}$

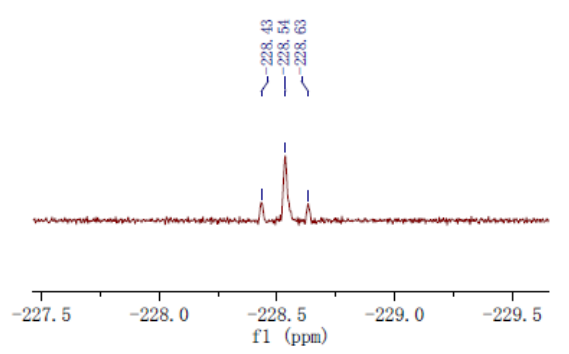

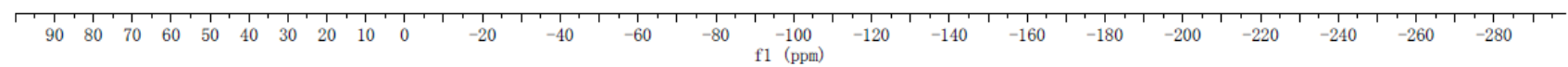

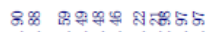

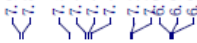

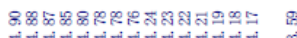

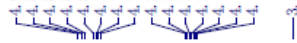
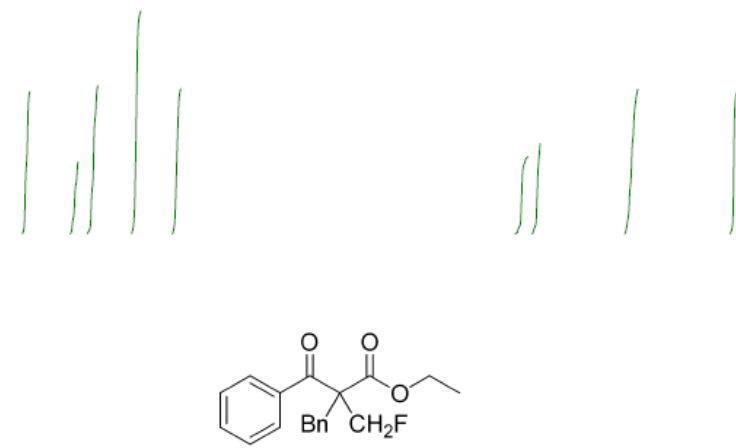

3y

$500 \mathrm{MHz} \mathrm{CDCl}_{3}$

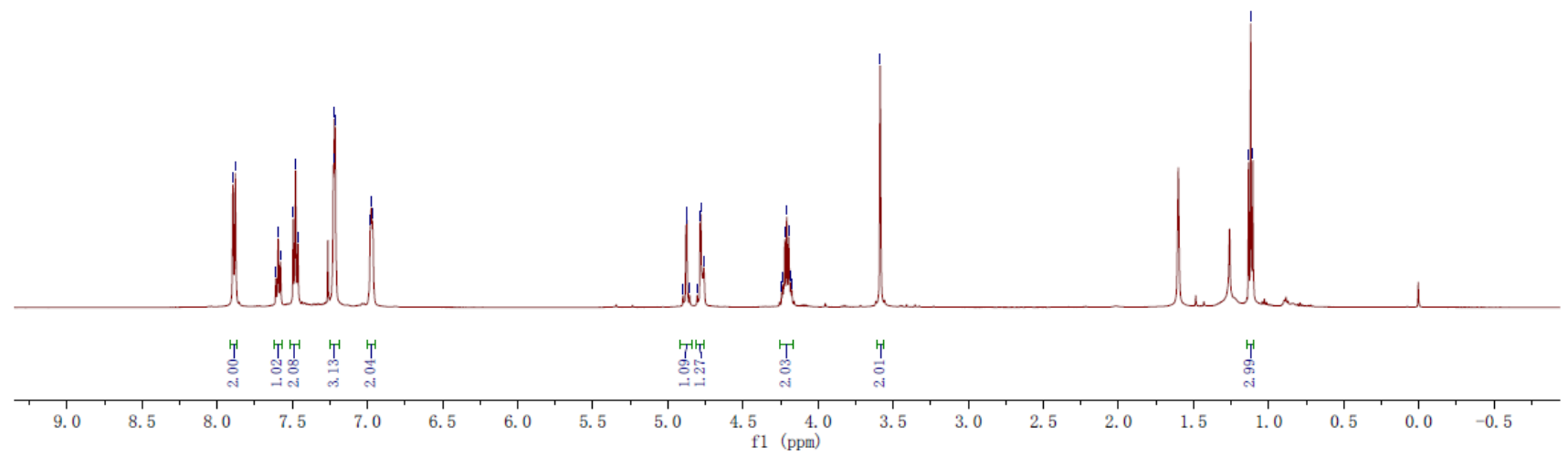




\begin{tabular}{|c|c|c|c|c|c|}
\hline $\begin{array}{l}88 \\
01\end{array}$ & 웜 & 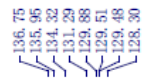 & 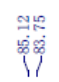 & 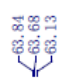 & के \\
\hline
\end{tabular}

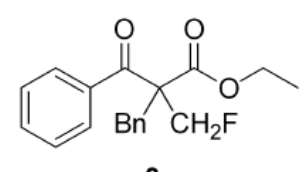

3y

$126 \mathrm{MHz}, \mathrm{CDCl}_{3}$
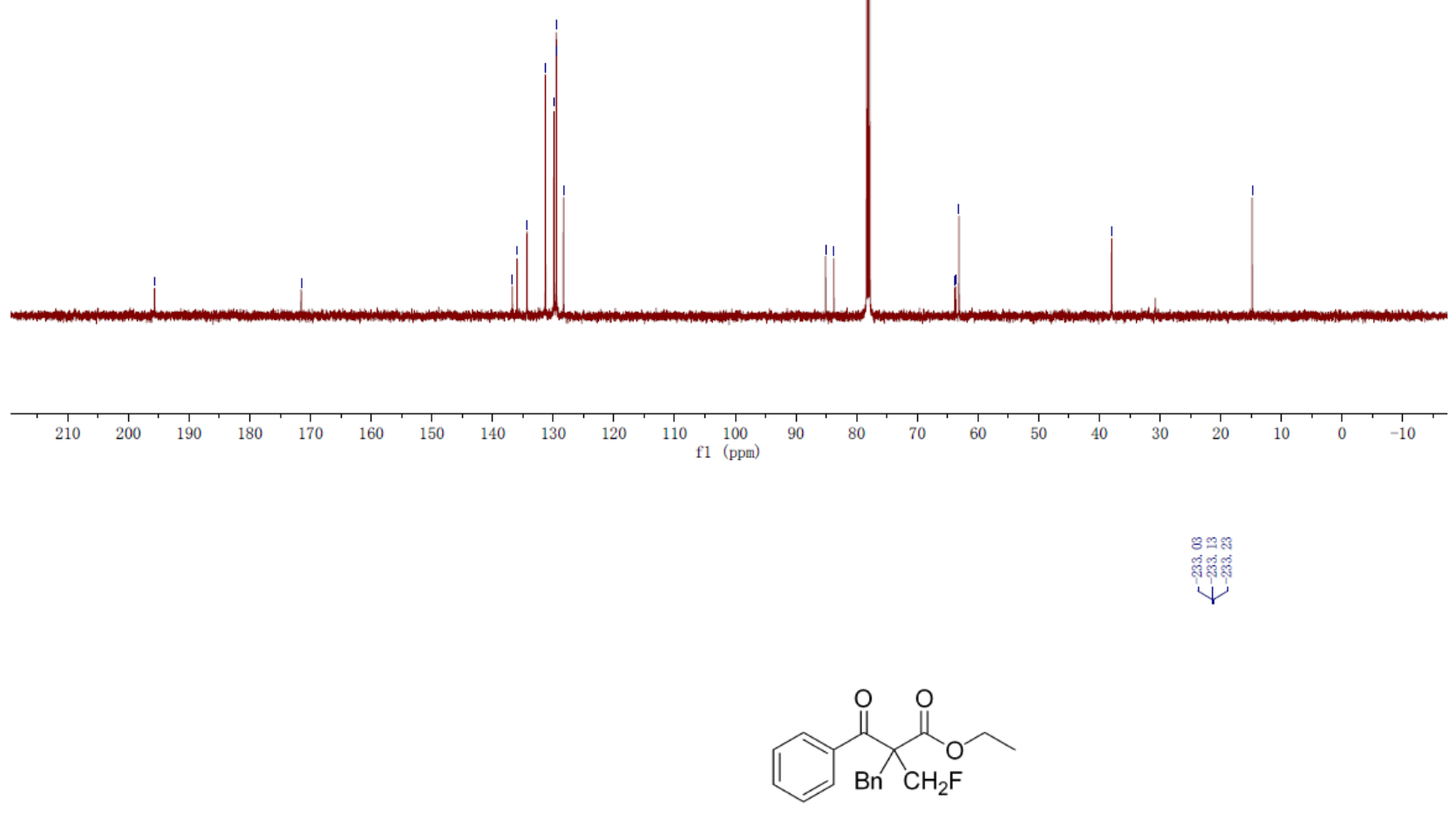

$3 y$

$470 \mathrm{MHz}, \mathrm{CDCl}_{3}$

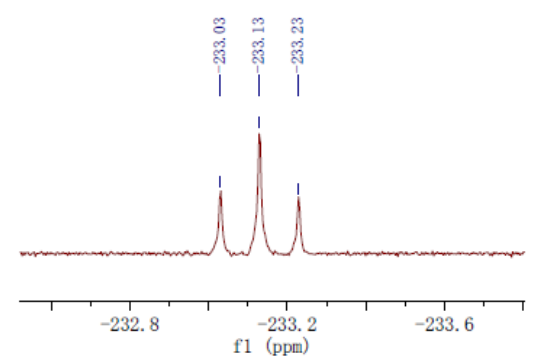

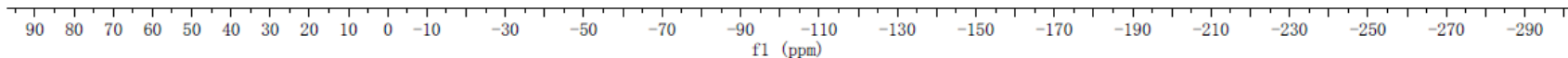




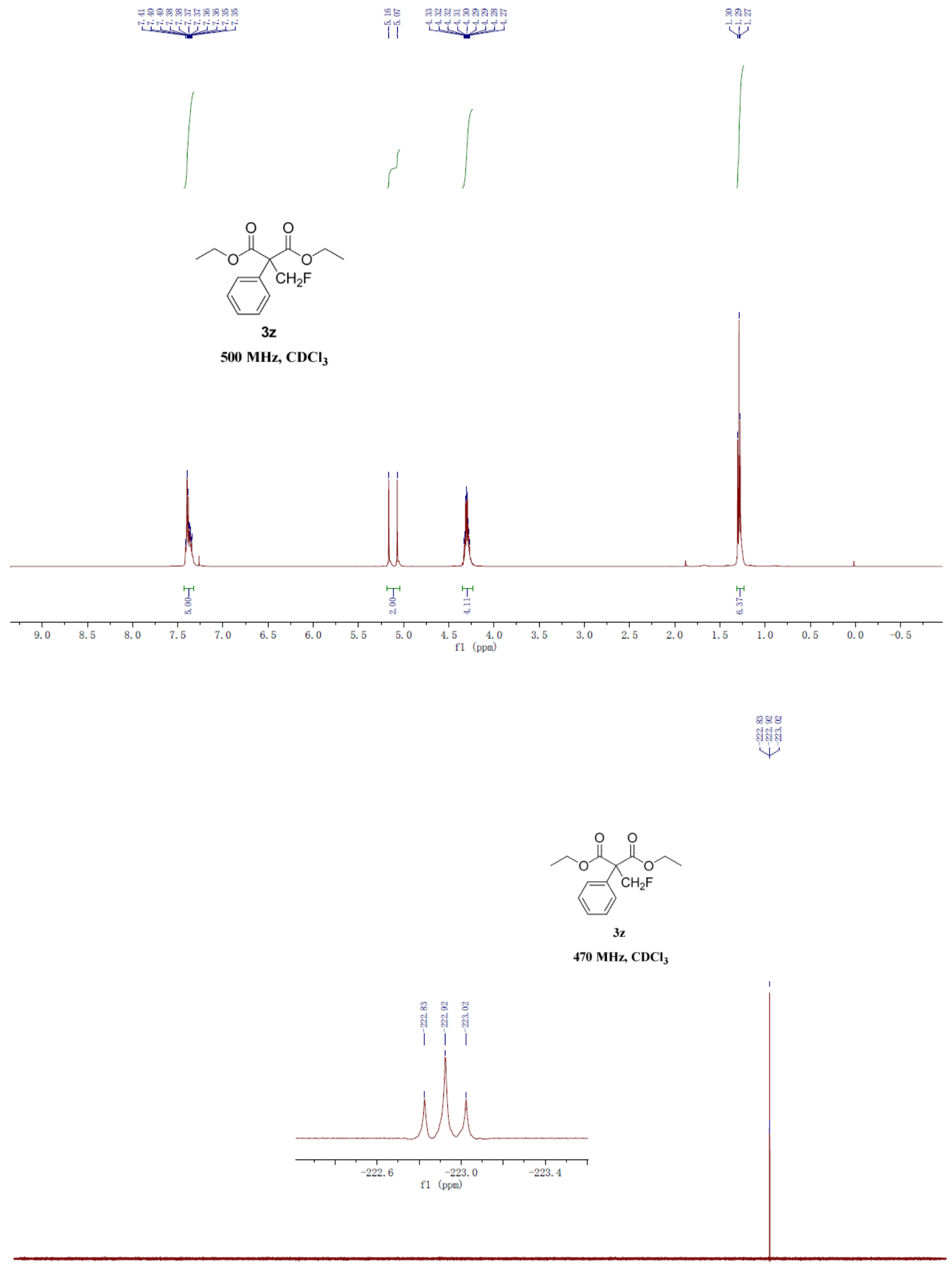

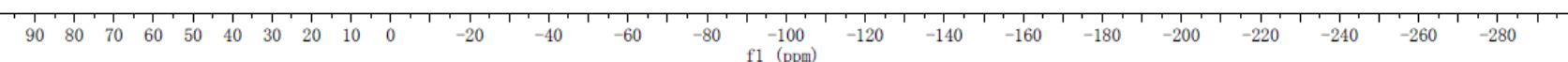


<smiles>CC1CC1</smiles>
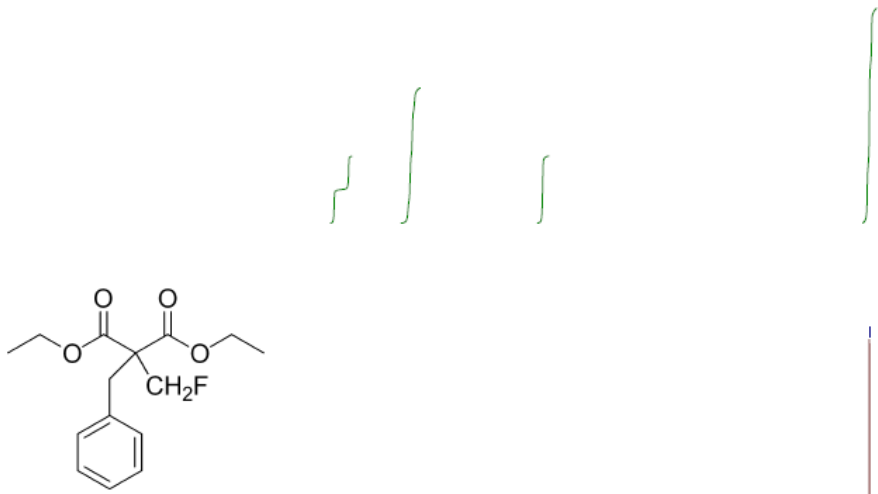

3aa

$500 \mathrm{MHz} \mathrm{CDCl}_{3}$
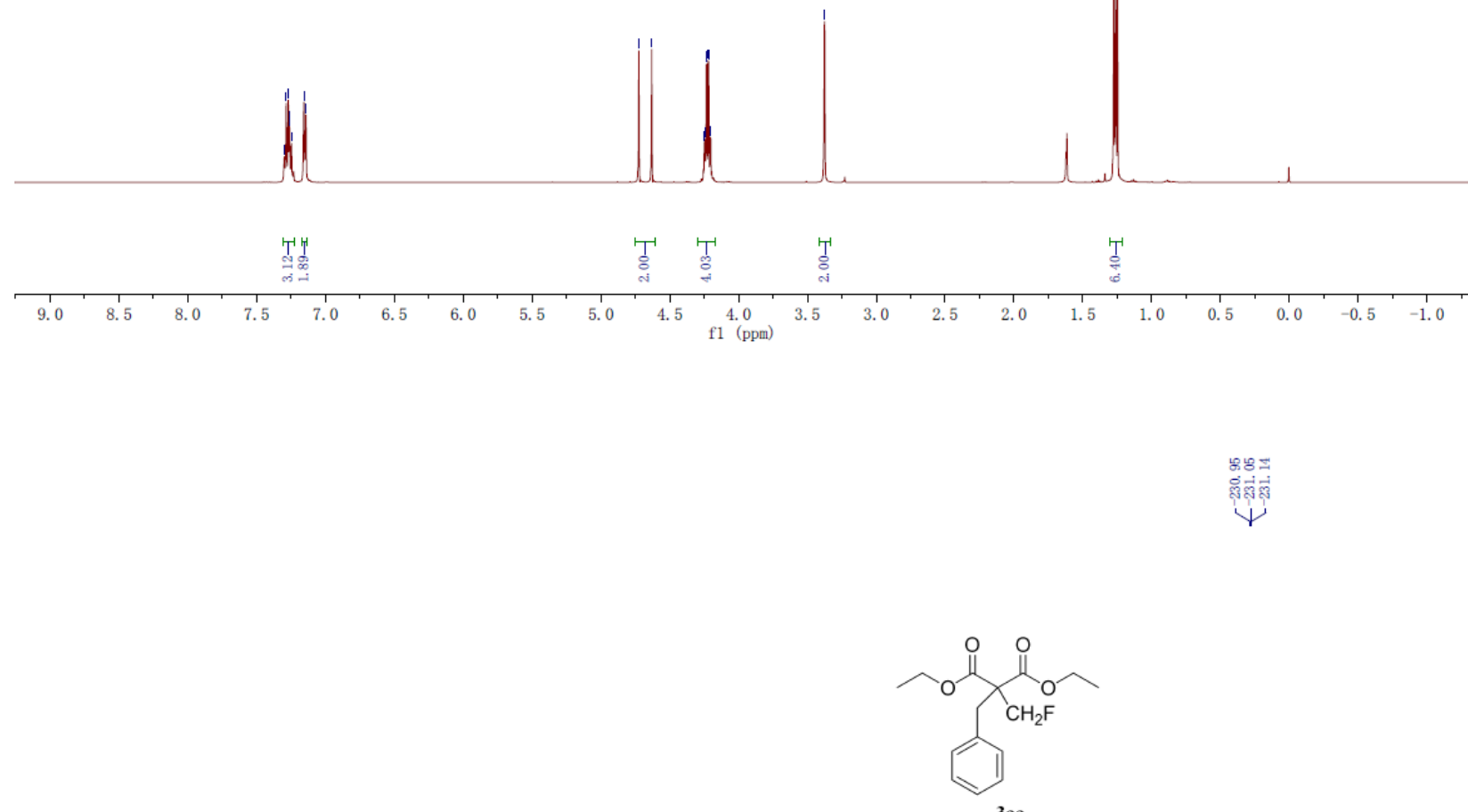

3aa

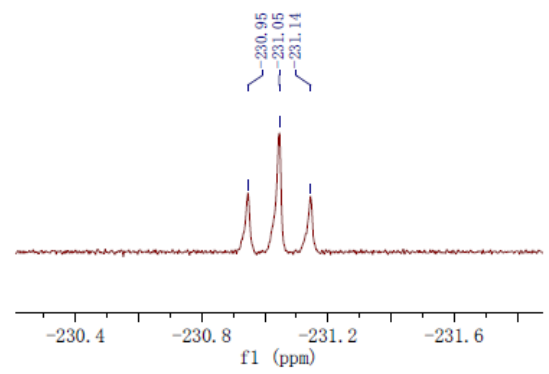

$470 \mathrm{MHz}, \mathrm{CDCl}_{3}$

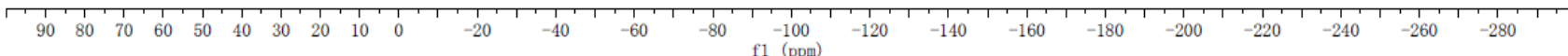



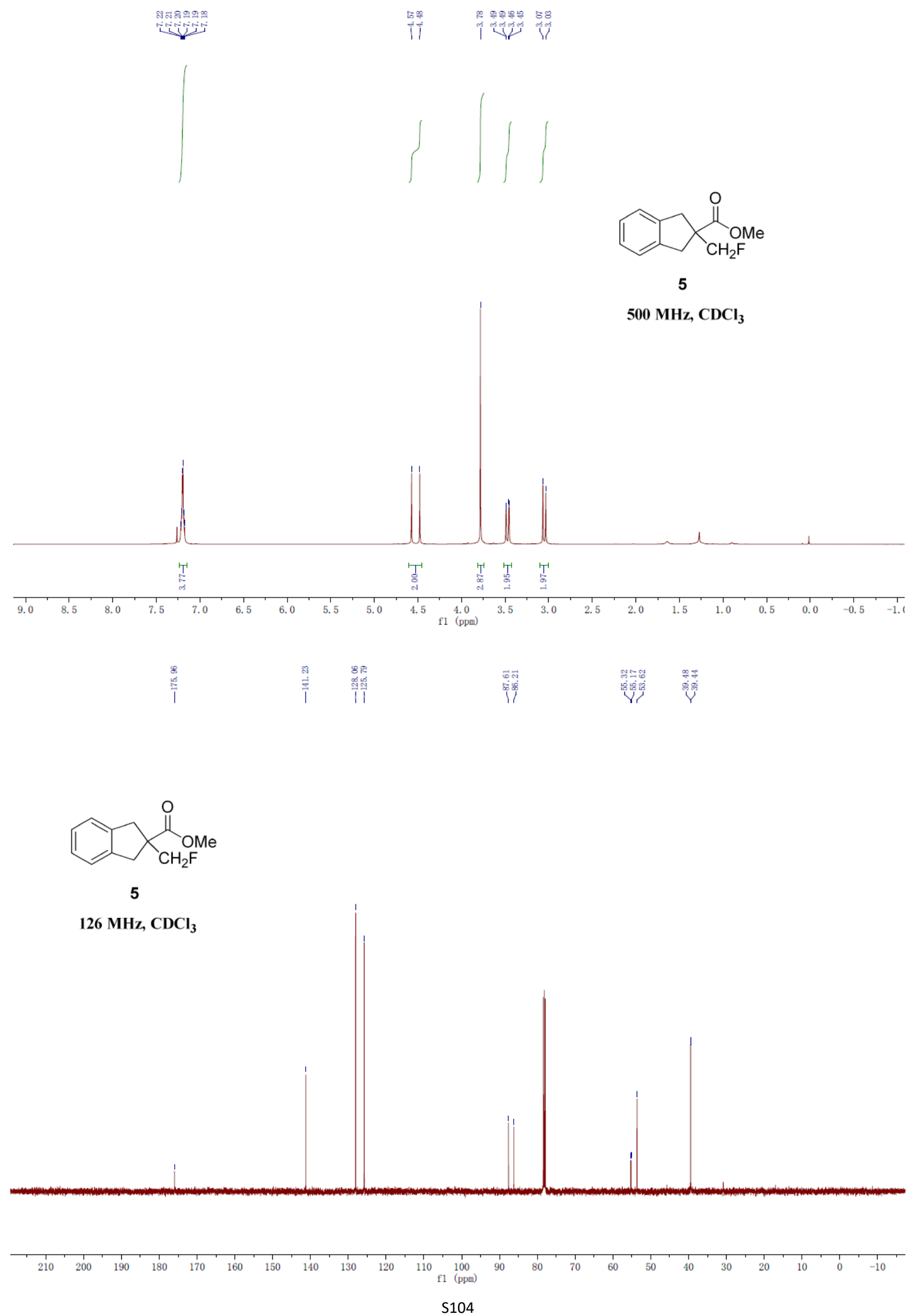


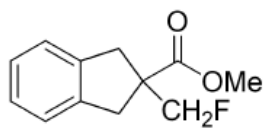

5

$470 \mathrm{MHz}, \mathrm{CDCl}_{3}$
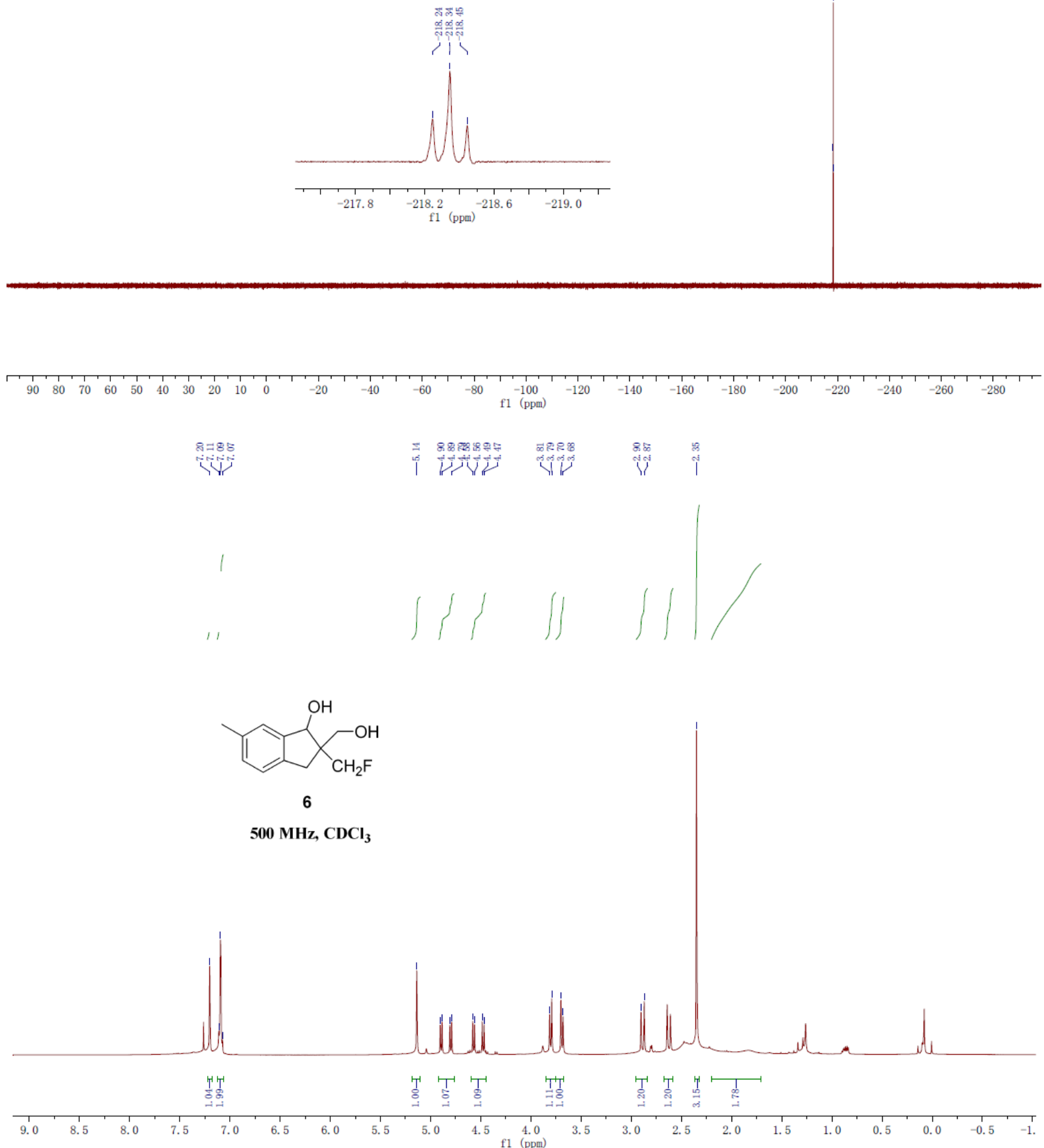


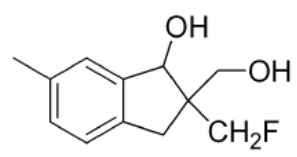

6

$126 \mathrm{MHz}^{\mathrm{CDCl}_{3}}$
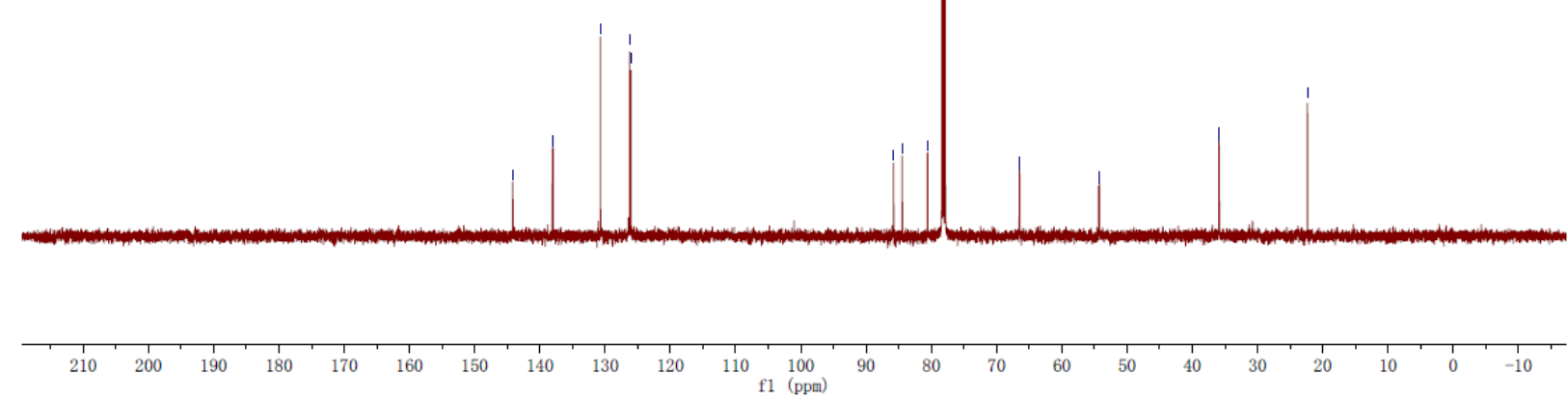

किल

ไัสำสำสำ

YW

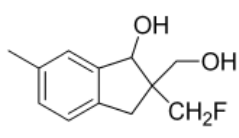

6

$\mathrm{CH}_{2}$

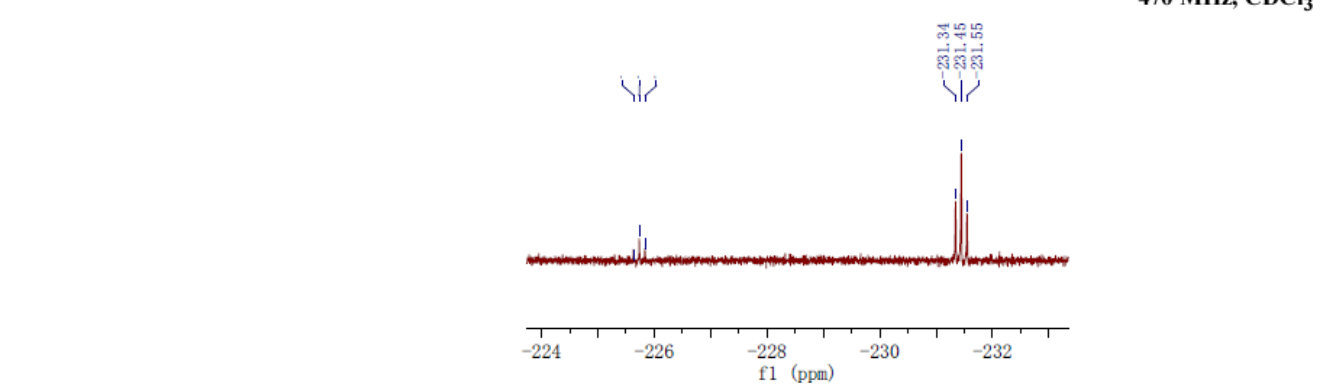

$470 \mathrm{MHz}, \mathrm{CDCl}_{3}$

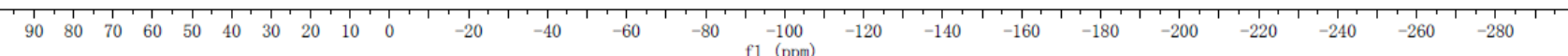

